\title{
Regio- and Diastereoselective Indium-Catalyzed Conia-Ene Reaction of ortho-Alkynyl Diketopiperazines to Access Fused Diketopiperazinoindolines
}

Hossein Zahedian Tejeneki, † Ali Nikbakht, † Saeed Balalaie, * †† Frank Rominger§

† Peptide Chemistry Research Center, K. N. Toosi University of Technology, P. O. Box 15875-4416, Tehran, Iran, bala-laie@kntu.ac.ir, Tel:+98-21-23064226, Fax: +98-21-22889403

$\ddagger$ Medical Biology Research Center, Kermanshah University of Medical Sciences, Kermanshah, Iran

§ Organisch-Chemisches Institut der Universität Heidelberg, Im Neuenheimer Feld 270, D-6912o Heidelberg, Germany

\section{Table of Contents:}

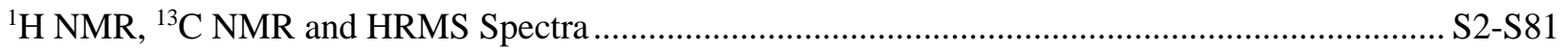

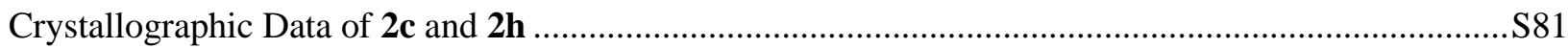




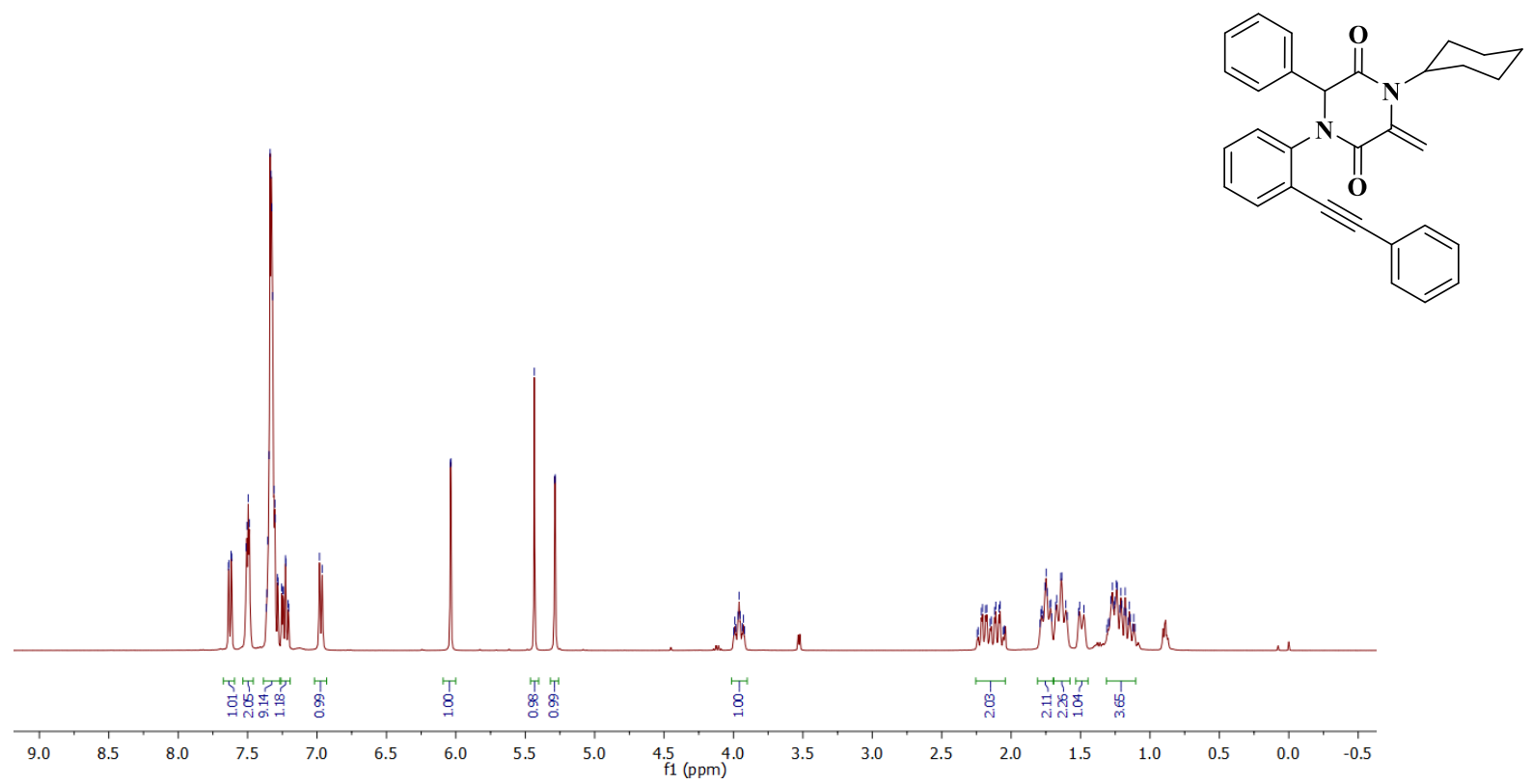

Figure S1. ${ }^{1} \mathrm{H} \mathrm{NMR}\left(400 \mathrm{MHz}, \mathrm{CDCl}_{3}\right) \mathbf{1 a}$

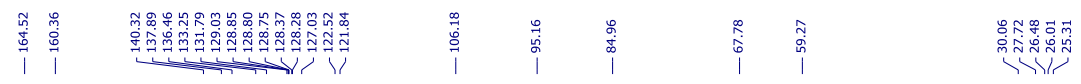
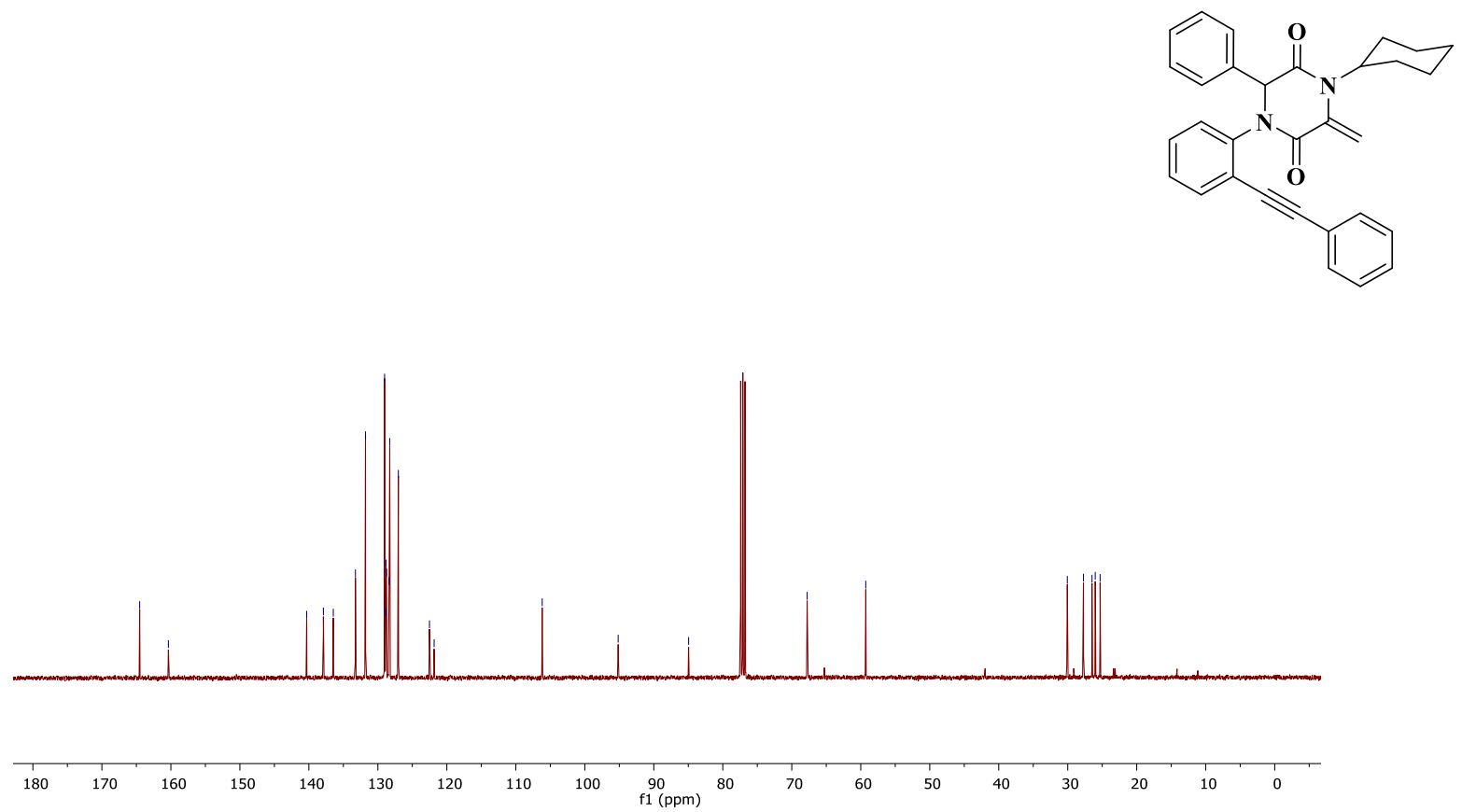

Figure $\mathrm{S} 2 .{ }^{13} \mathrm{C}$ NMR $\left(100 \mathrm{MHz}, \mathrm{CDCl}_{3}\right)$ 1a 


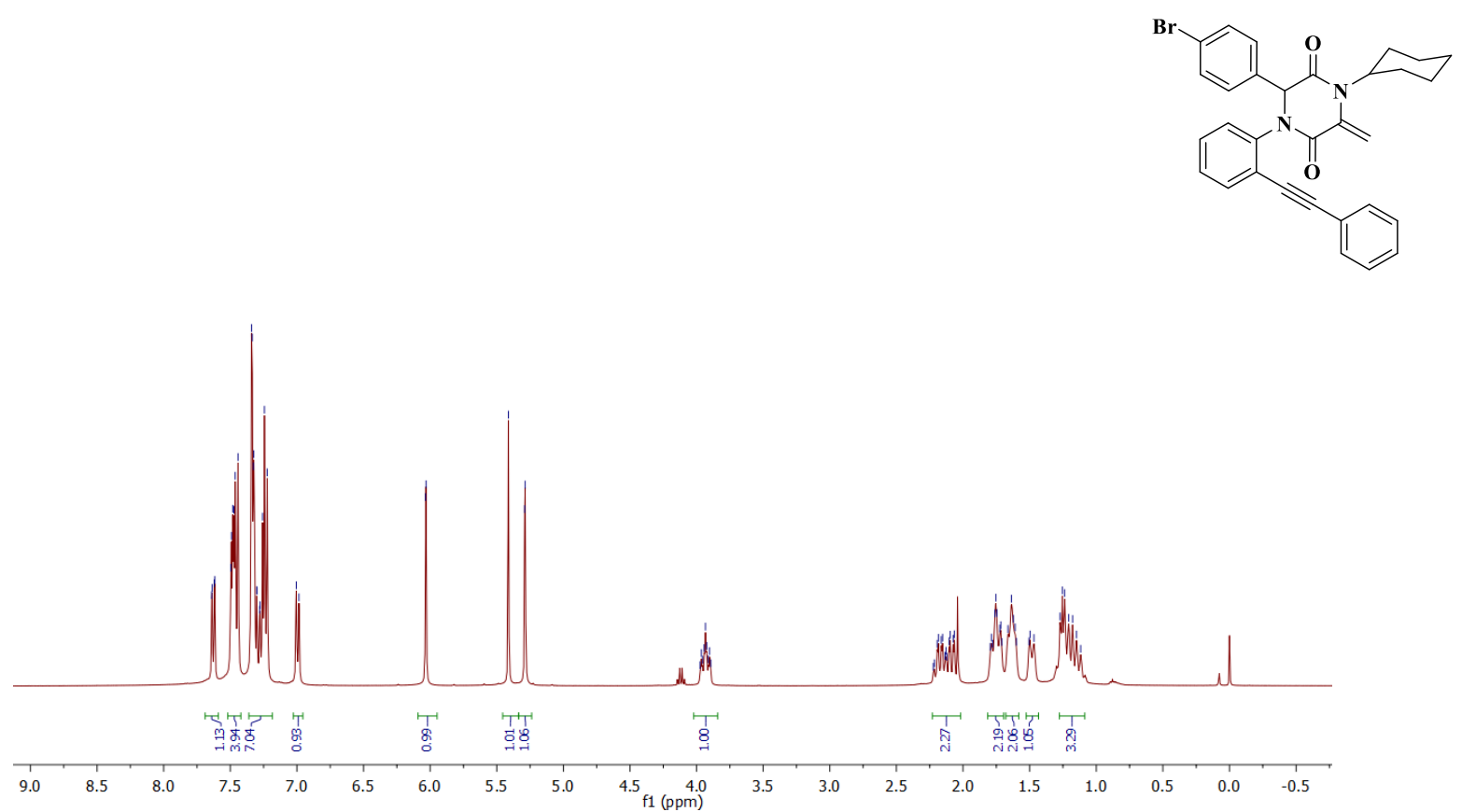

Figure S3. ${ }^{1} \mathrm{H}$ NMR( $\left(400 \mathrm{MHz}, \mathrm{CDCl}_{3}\right) \mathbf{1 b}$
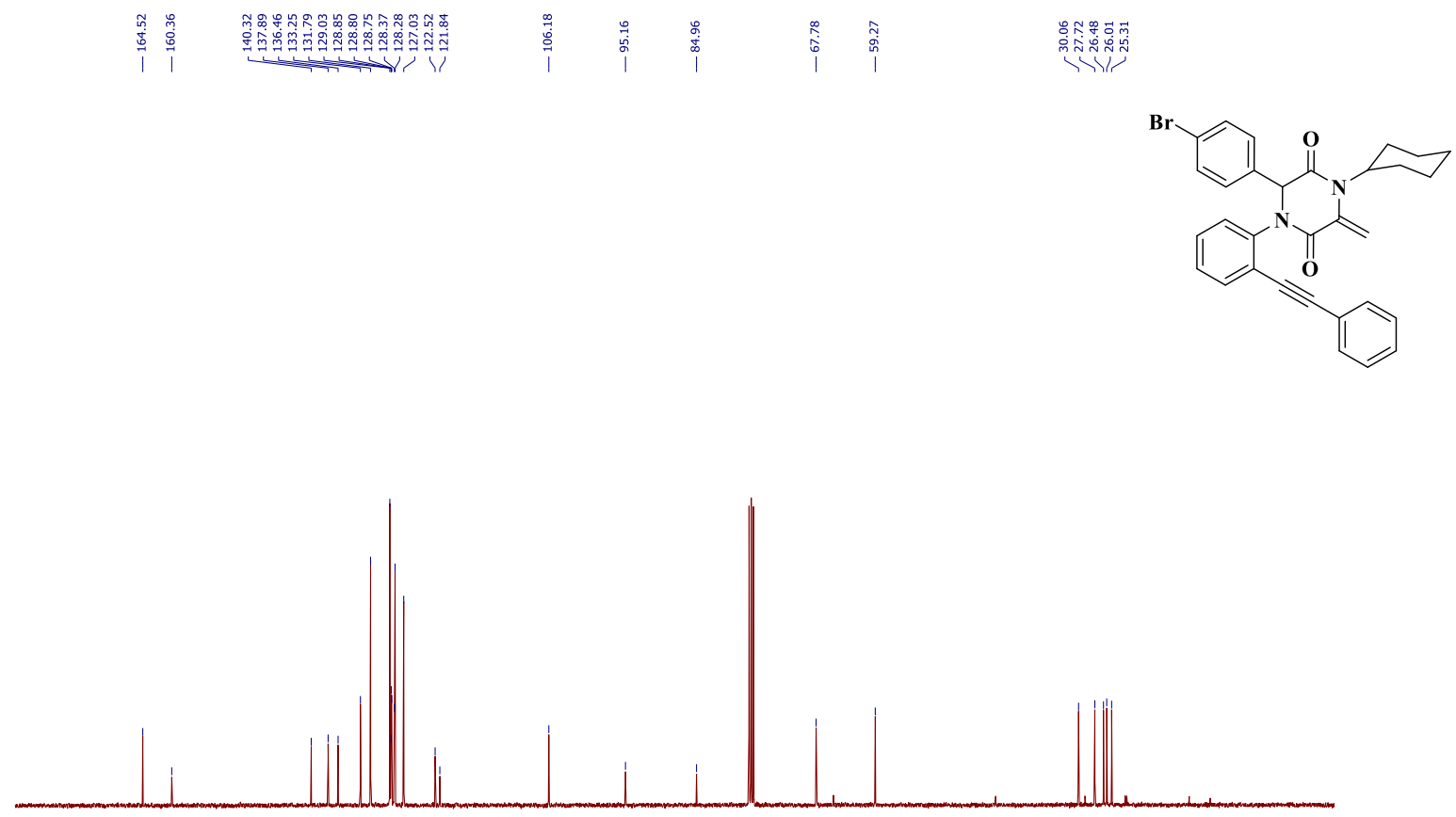

180
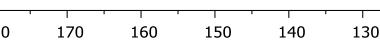

90

Figure S4. ${ }^{13} \mathrm{C} \mathrm{NMR}\left(100 \mathrm{MHz}, \mathrm{CDCl}_{3}\right) \mathbf{1 b}$ 

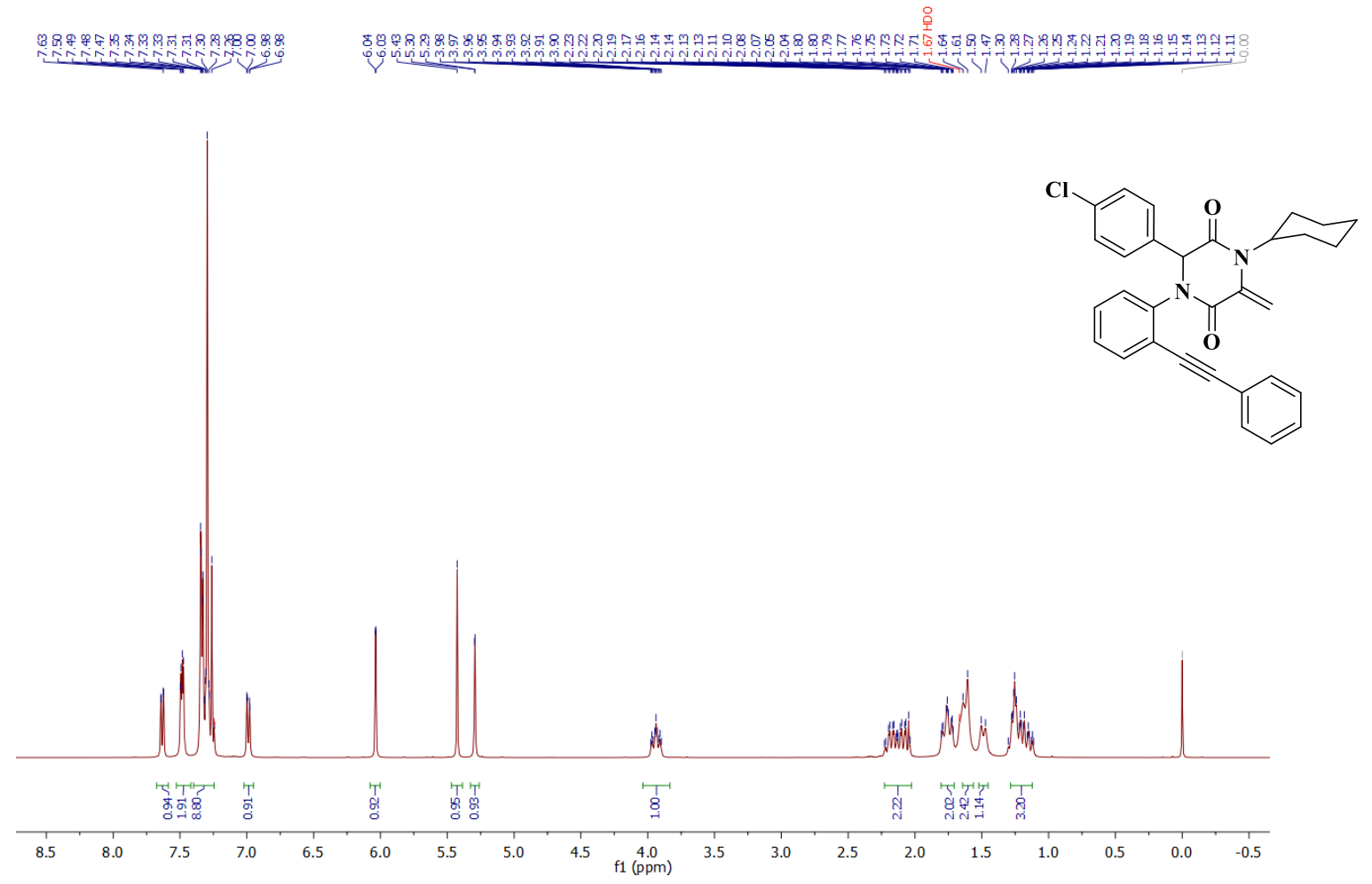

Figure S5. ${ }^{1} \mathrm{H}$ NMR $\left(400 \mathrm{MHz}, \mathrm{CDCl}_{3}\right) \mathbf{1 c}$

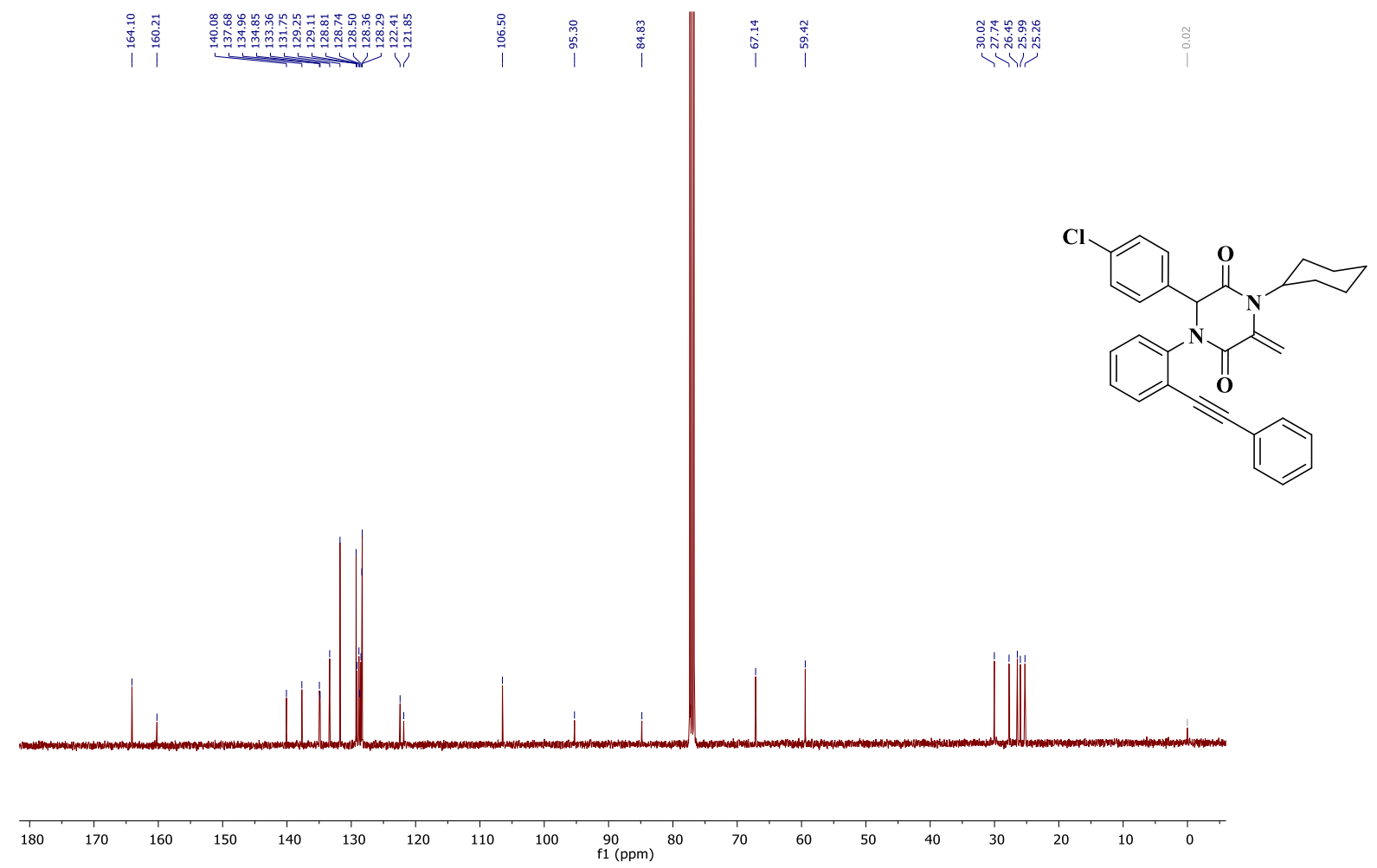

Figure S6. ${ }^{13} \mathrm{C} \mathrm{NMR}\left(100 \mathrm{MHz}, \mathrm{CDCl}_{3}\right) \mathbf{1 c}$ 


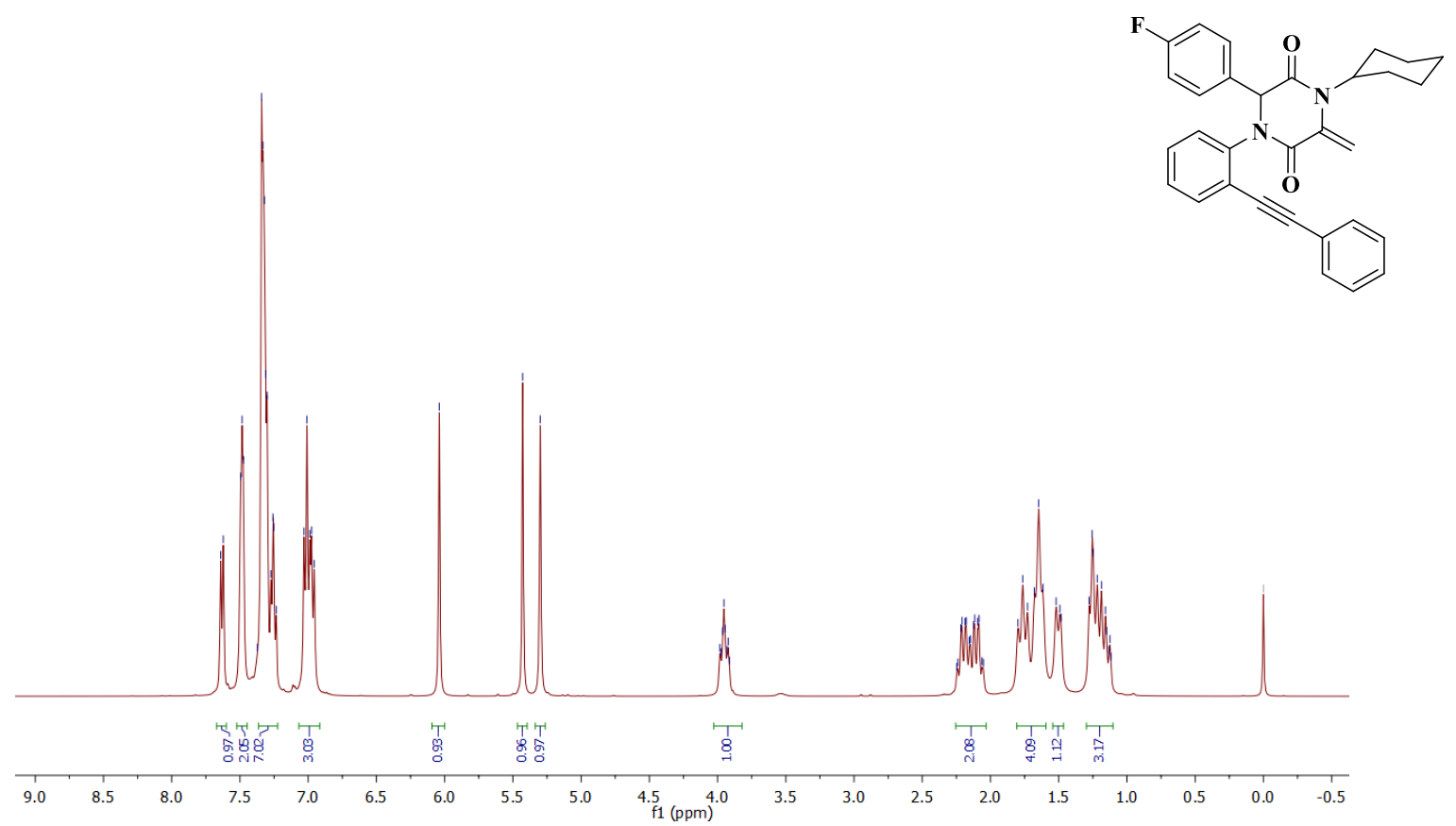

Figure S7. ${ }^{1} \mathrm{H}$ NMR (400 MHz, $\left.\mathrm{CDCl}_{3}\right)$ 1d

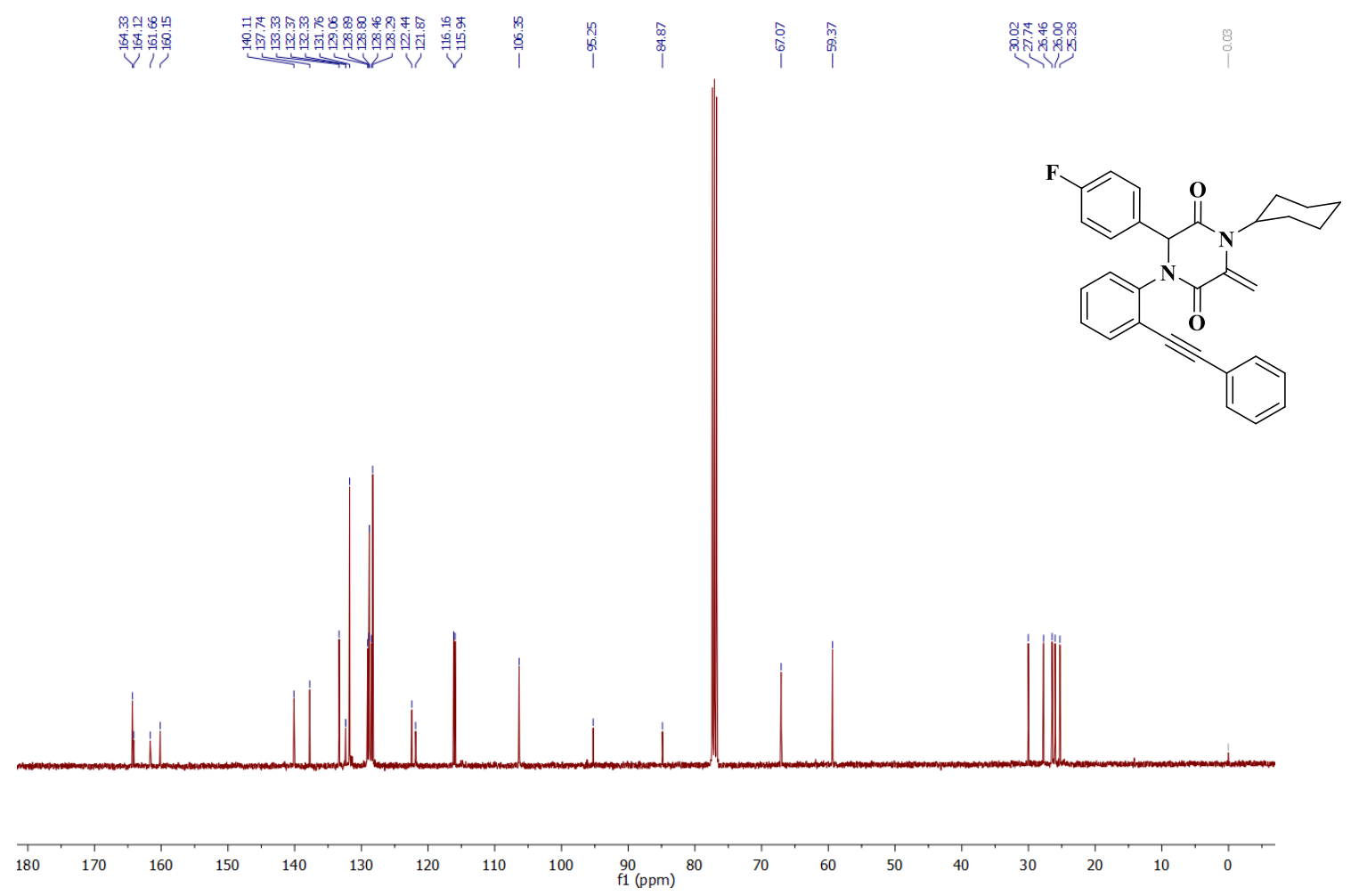

Figure S8. ${ }^{13} \mathrm{C}$ NMR $\left(100 \mathrm{MHz}, \mathrm{CDCl}_{3}\right) \mathbf{1 d}$ 


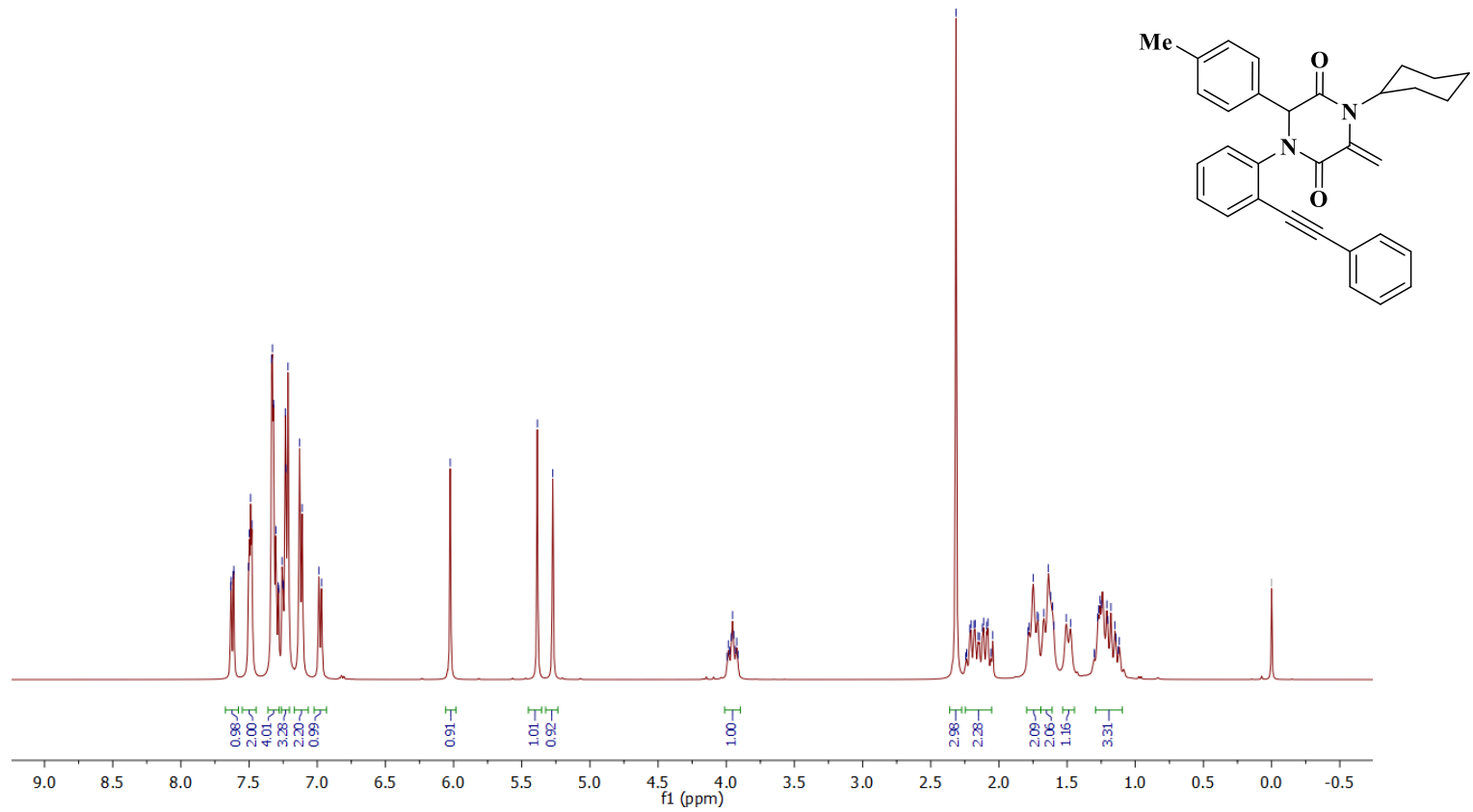

Figure S9. ${ }^{1} \mathrm{H}$ NMR (400 MHz, $\left.\mathrm{CDCl}_{3}\right) \mathbf{1 e}$

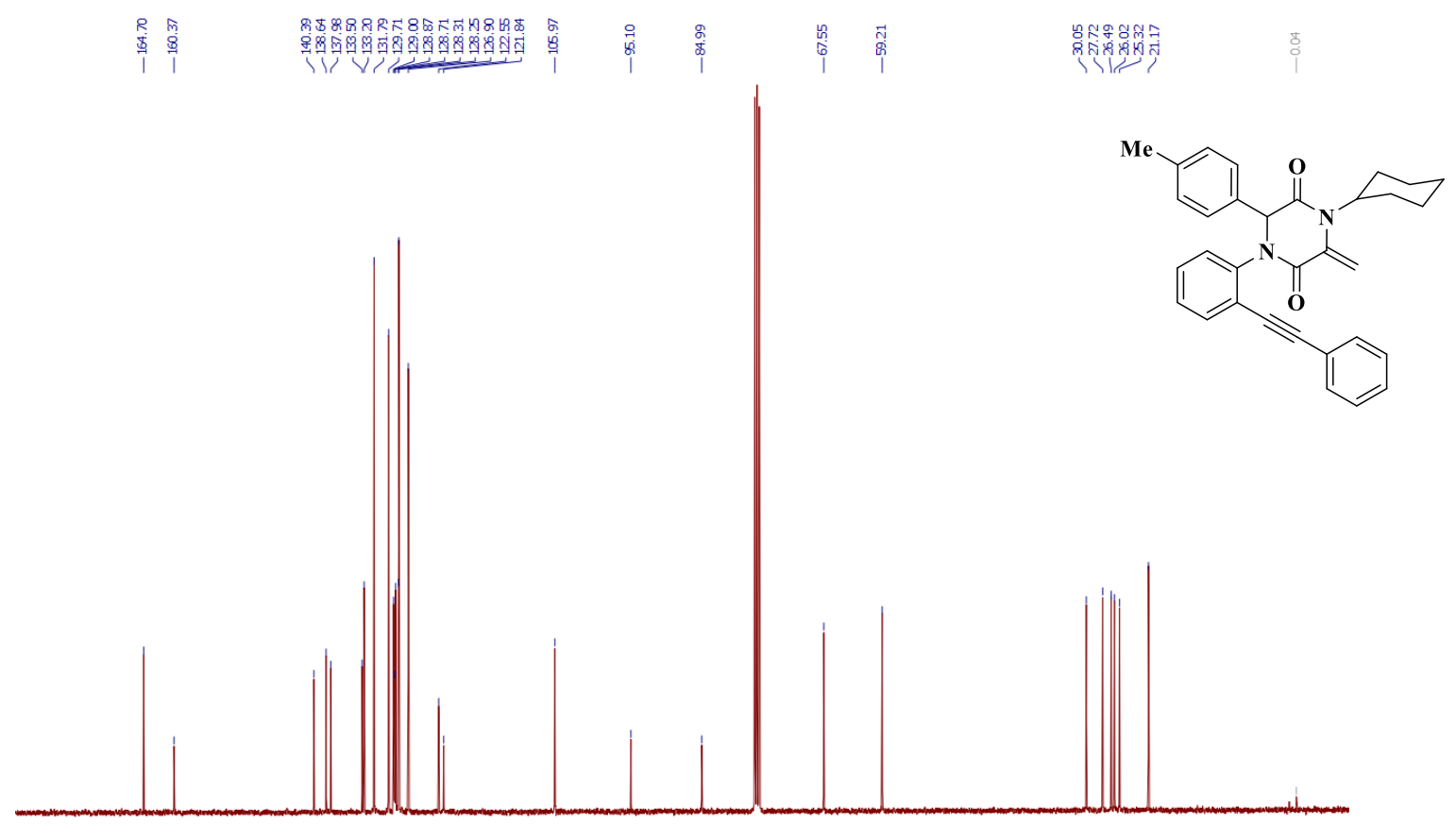

Figure $\mathrm{S} 10 .{ }^{13} \mathrm{C} \mathrm{NMR}\left(100 \mathrm{MHz}, \mathrm{CDCl}_{3}\right) \mathbf{1 e}$ 


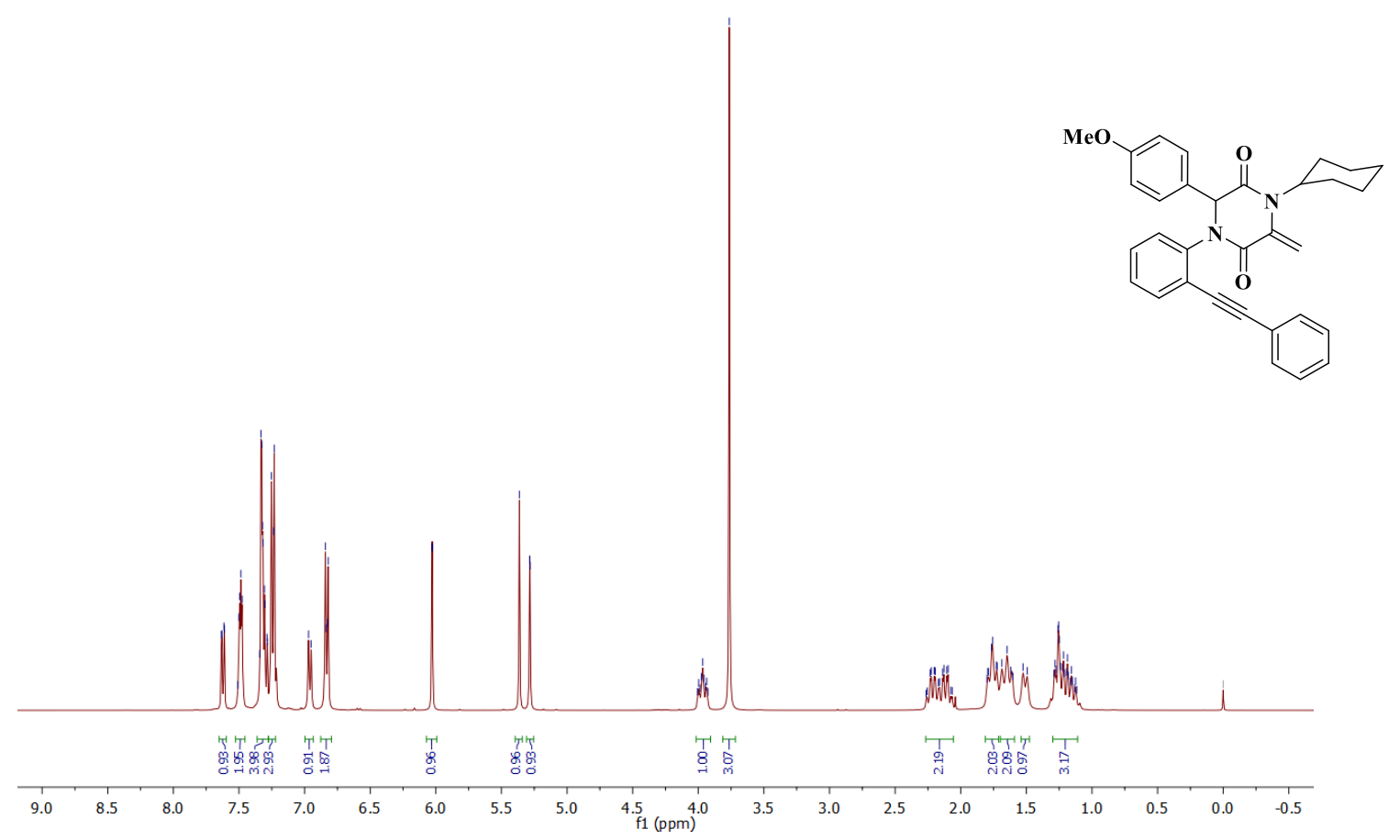

Figure S11. ${ }^{1} \mathrm{H}$ NMR(400 MHz, $\left.\mathrm{CDCl}_{3}\right)$ 1f

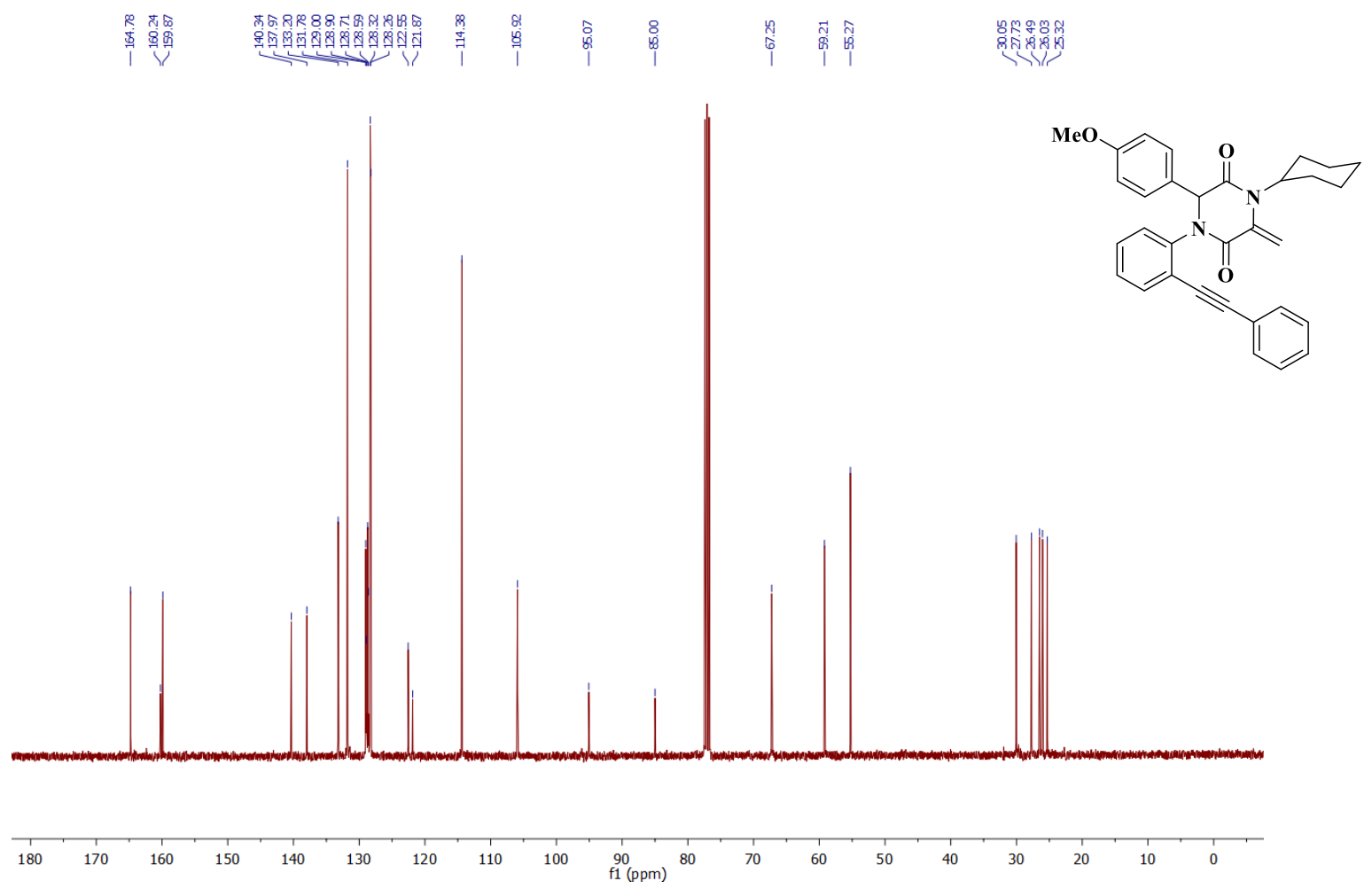

Figure $\mathrm{S} 12 .{ }^{13} \mathrm{C} \mathrm{NMR}\left(100 \mathrm{MHz}, \mathrm{CDCl}_{3}\right) \mathbf{1 f}$ 


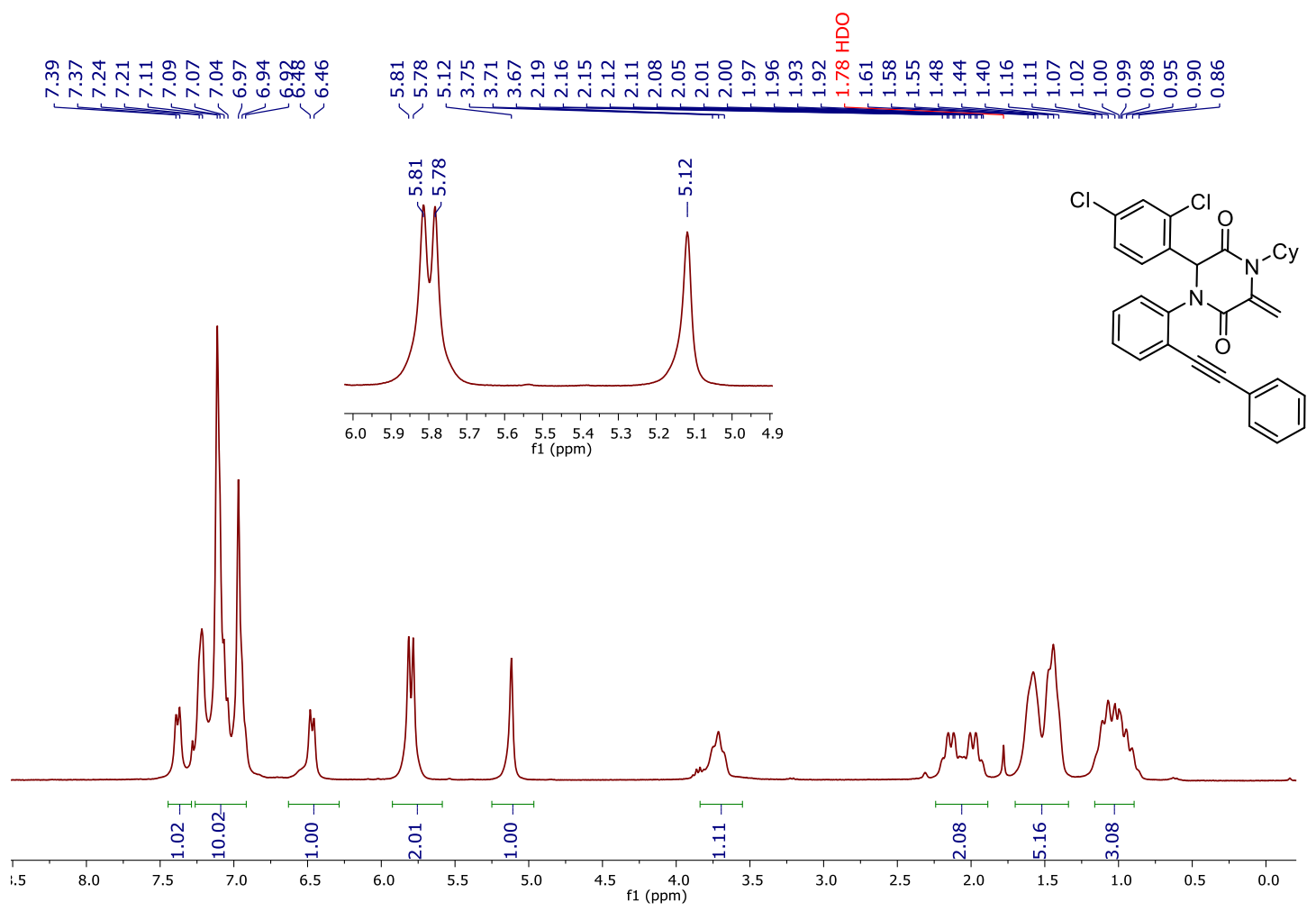

Figure S13. ${ }^{1} \mathrm{H}$ NMR(300 MHz, $\left.\mathrm{CDCl}_{3}\right) \mathbf{1 g}$
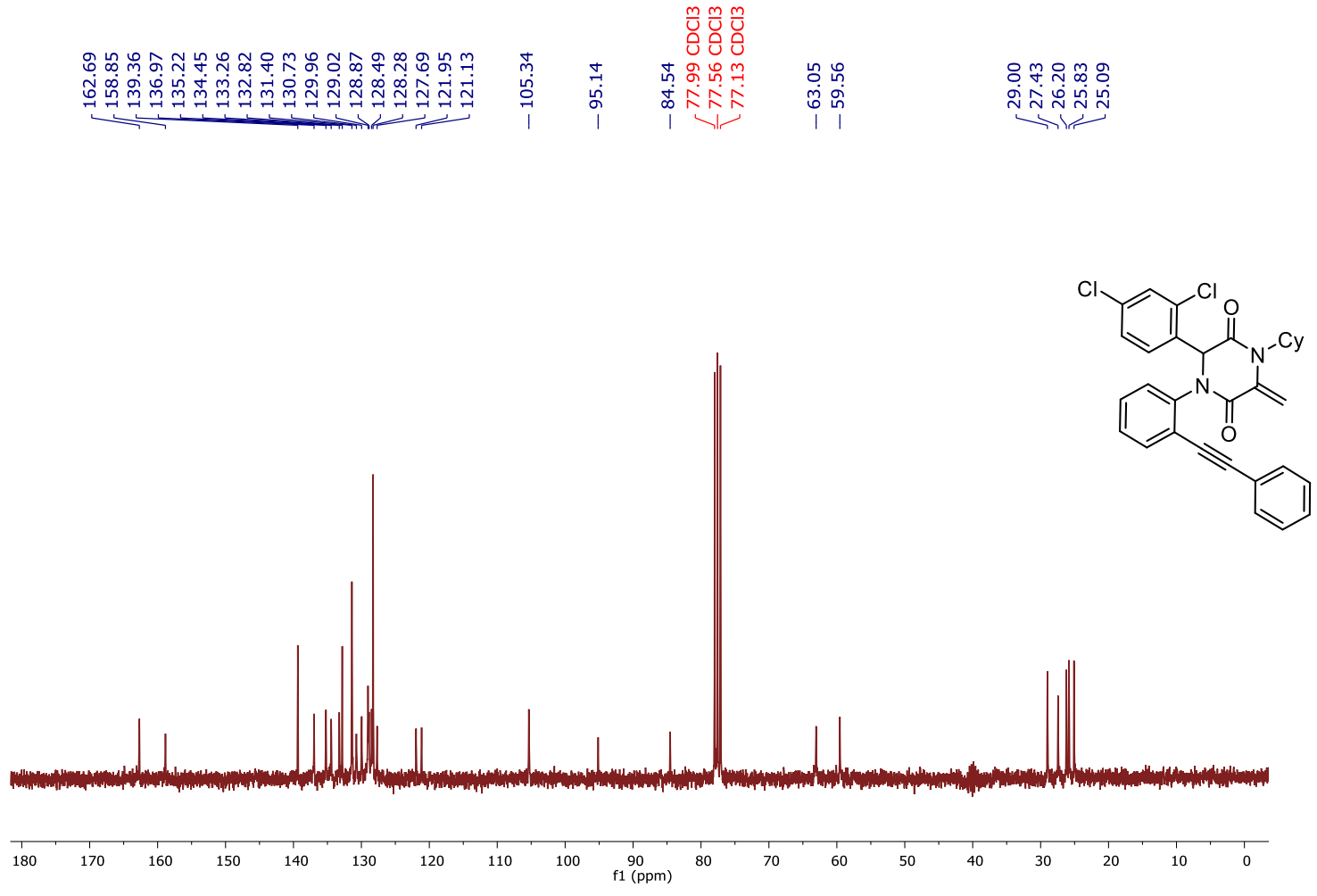

Figure S14. ${ }^{13} \mathrm{C} \mathrm{NMR}\left(75 \mathrm{MHz}, \mathrm{CDCl}_{3}\right) \mathbf{1 g}$ 


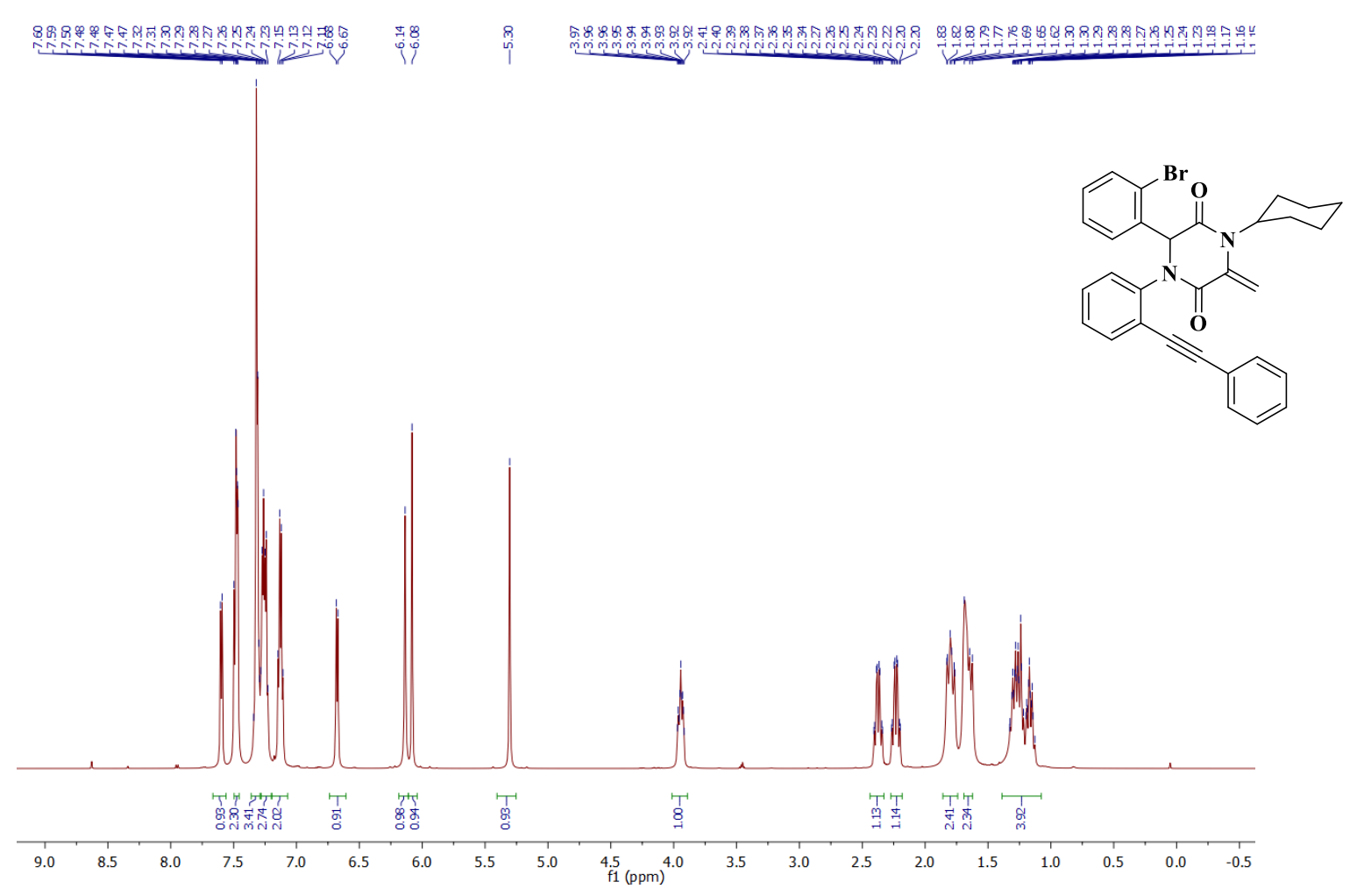

Figure S15. ${ }^{1} \mathrm{H}$ NMR(600 MHz, $\left.\mathrm{CDCl}_{3}\right) \mathbf{1 h}$
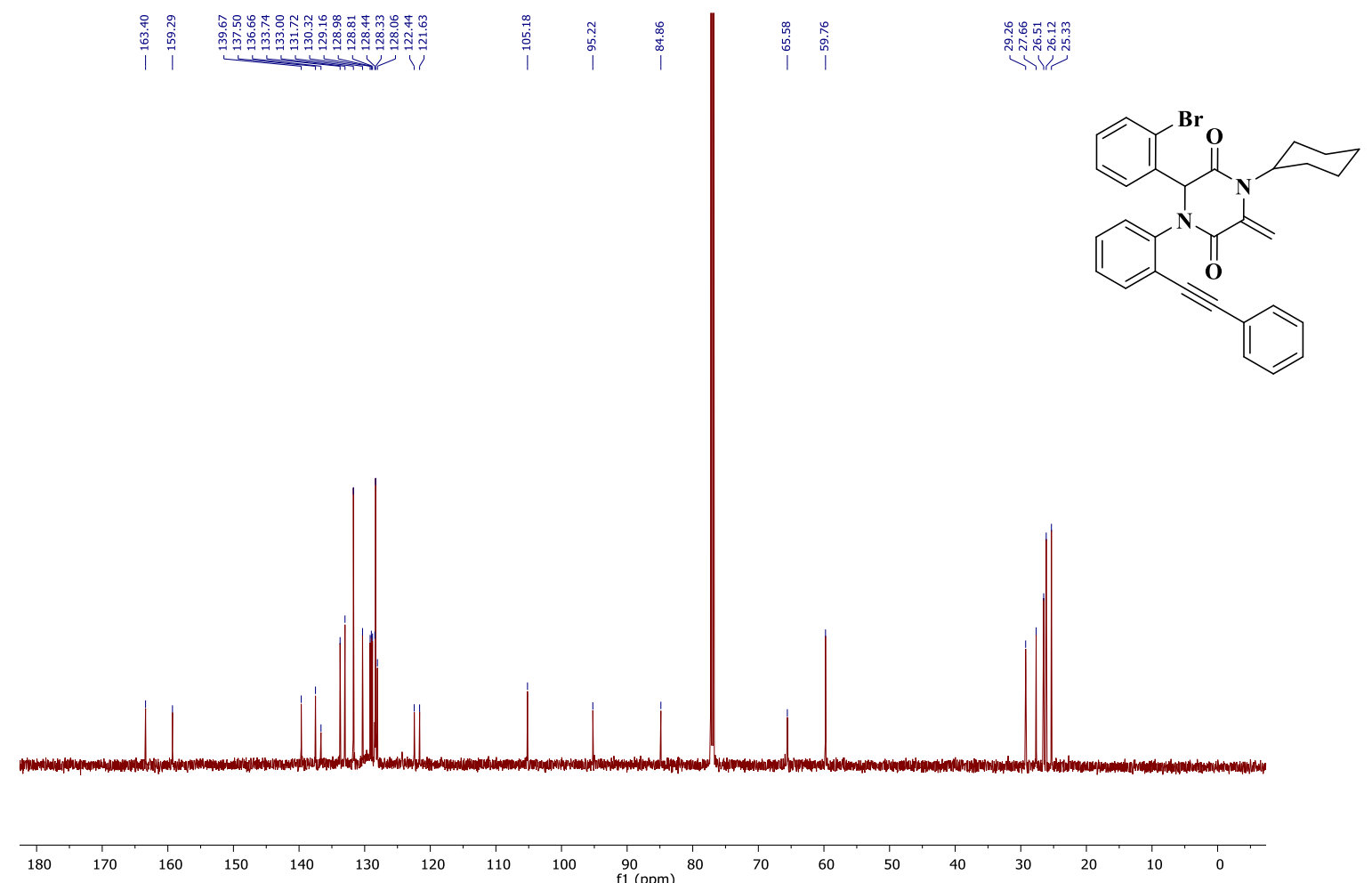

Figure S16. ${ }^{13} \mathrm{C} \mathrm{NMR}\left(150 \mathrm{MHz}, \mathrm{CDCl}_{3}\right) \mathbf{1 h}$ 

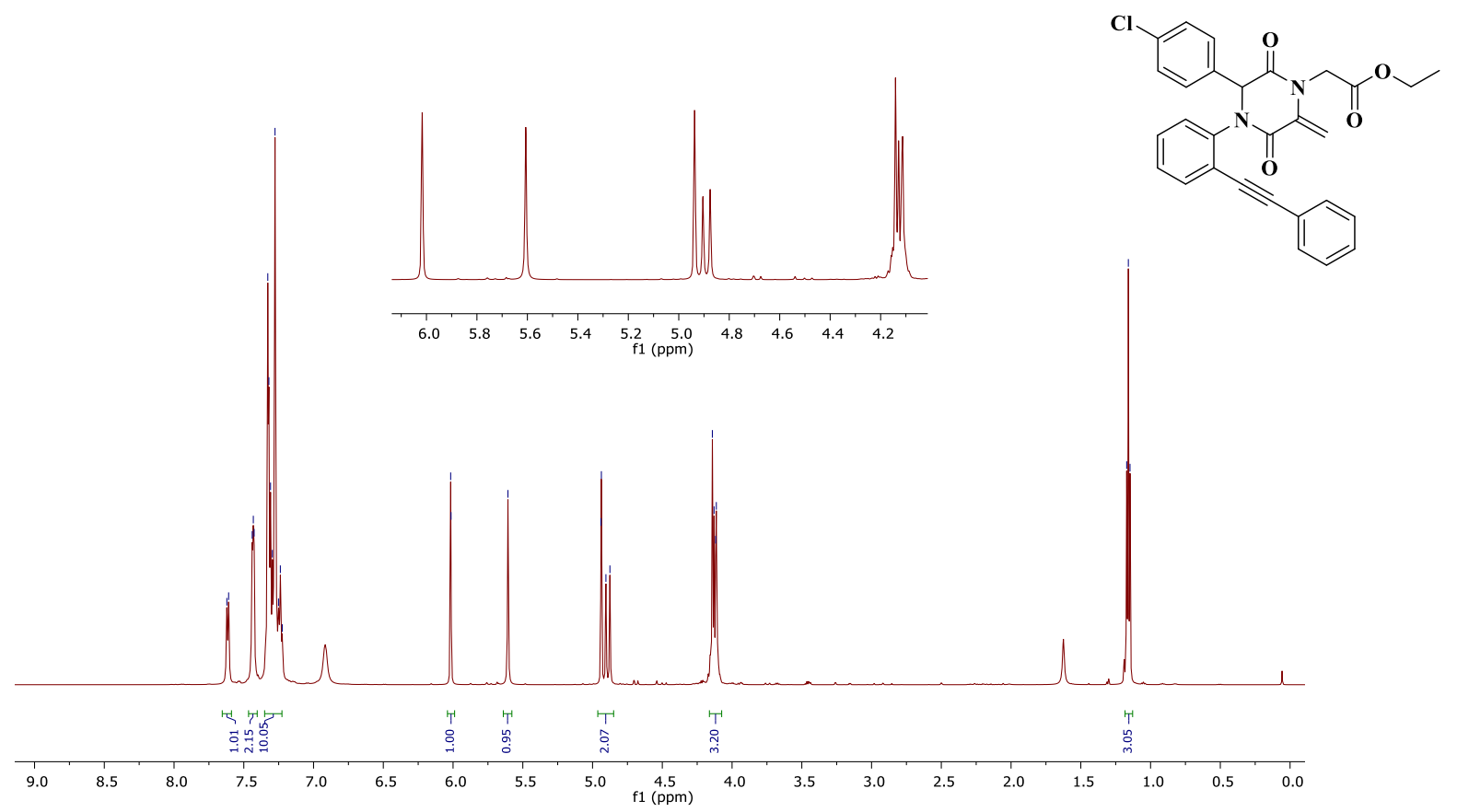

Figure S17. ${ }^{1} \mathrm{H}$ NMR(600 MHz, $\left.\mathrm{CDCl}_{3}\right) \mathbf{1 i}$

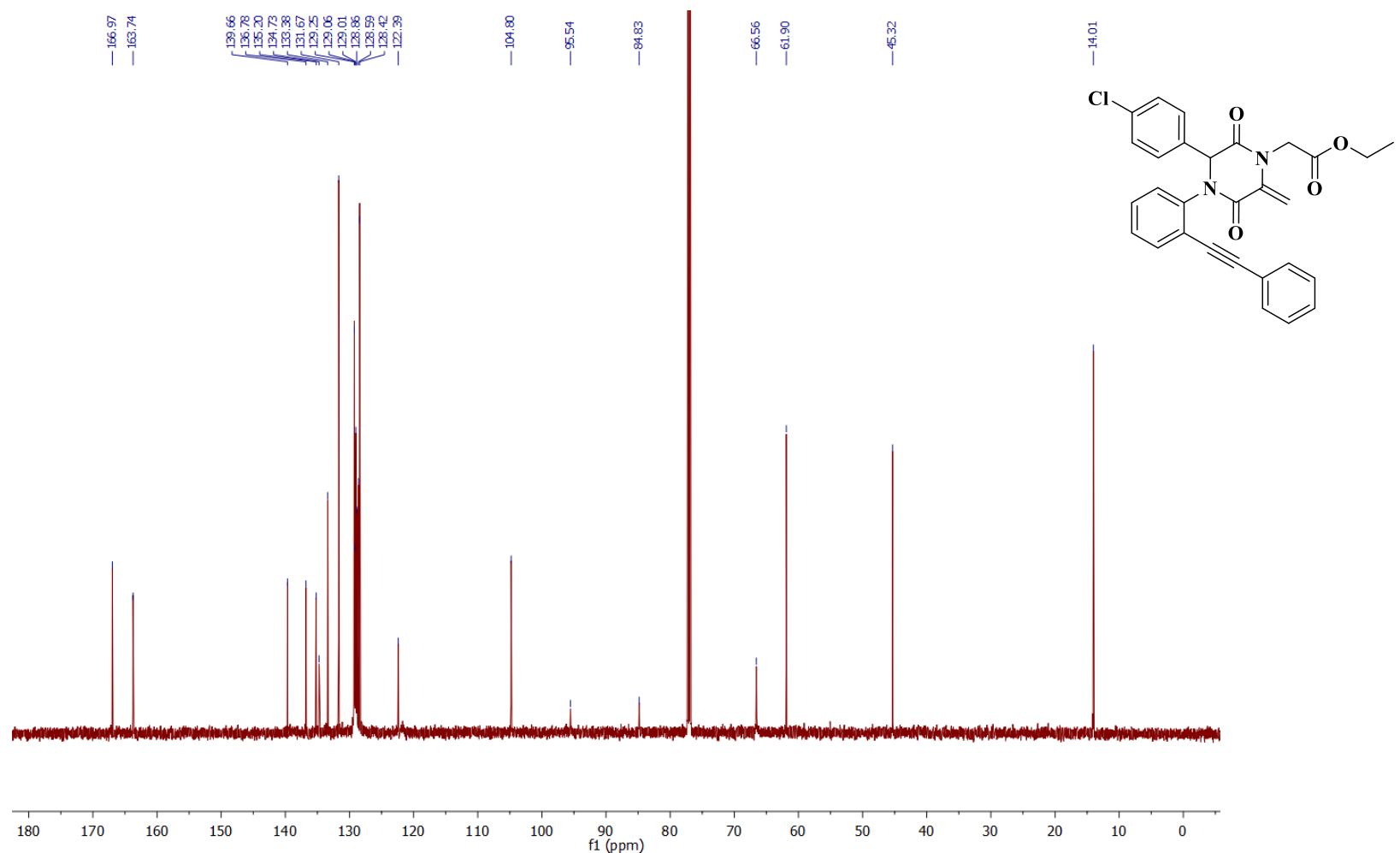

Figure S18. ${ }^{13} \mathrm{C}$ NMR $\left(150 \mathrm{MHz}, \mathrm{CDCl}_{3}\right) \mathbf{1 i}$ 
8. लि8
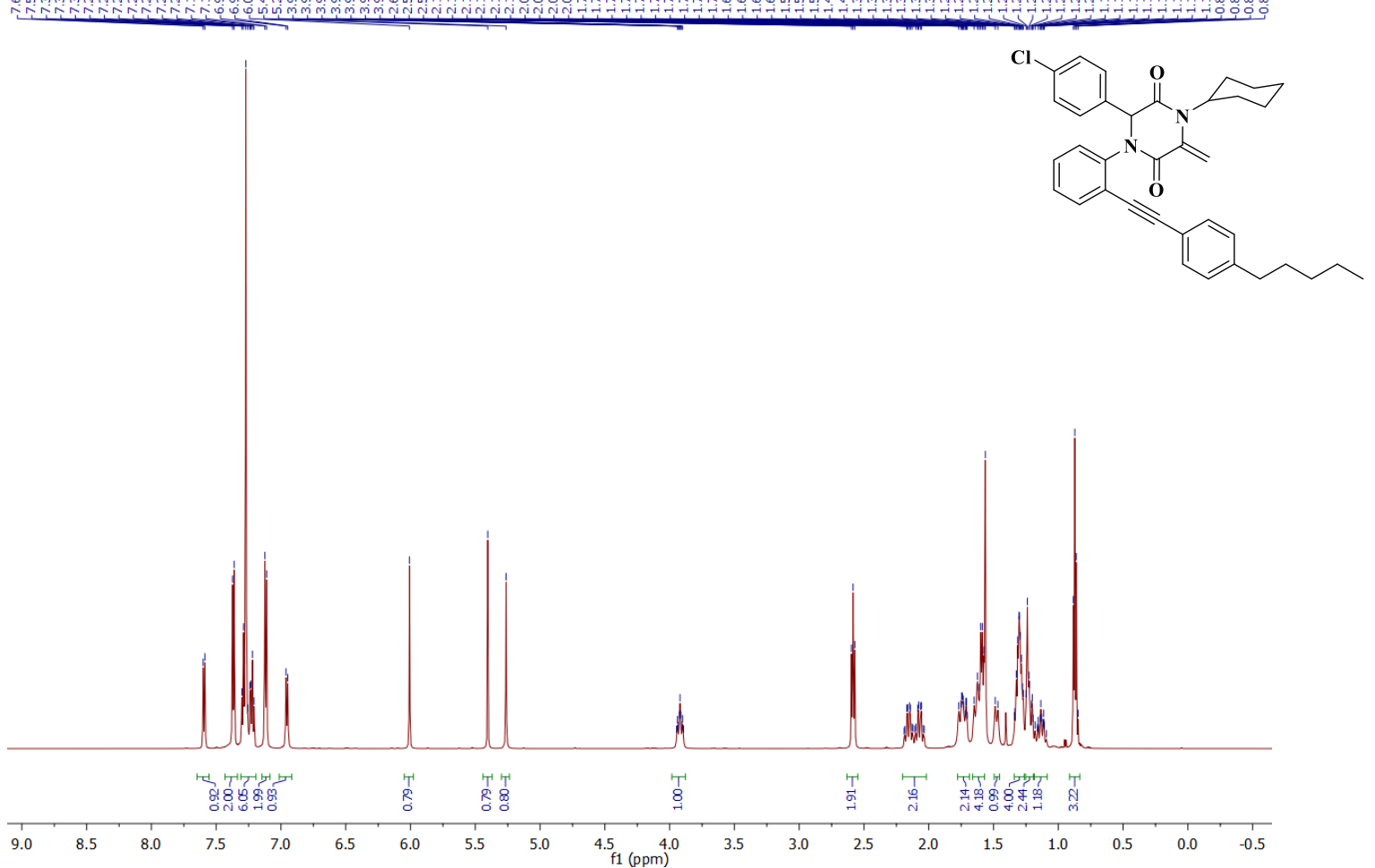

Figure S19. ${ }^{1} \mathrm{H}$ NMR(600 MHz, $\left.\mathrm{CDCl}_{3}\right) \mathbf{1 j}$

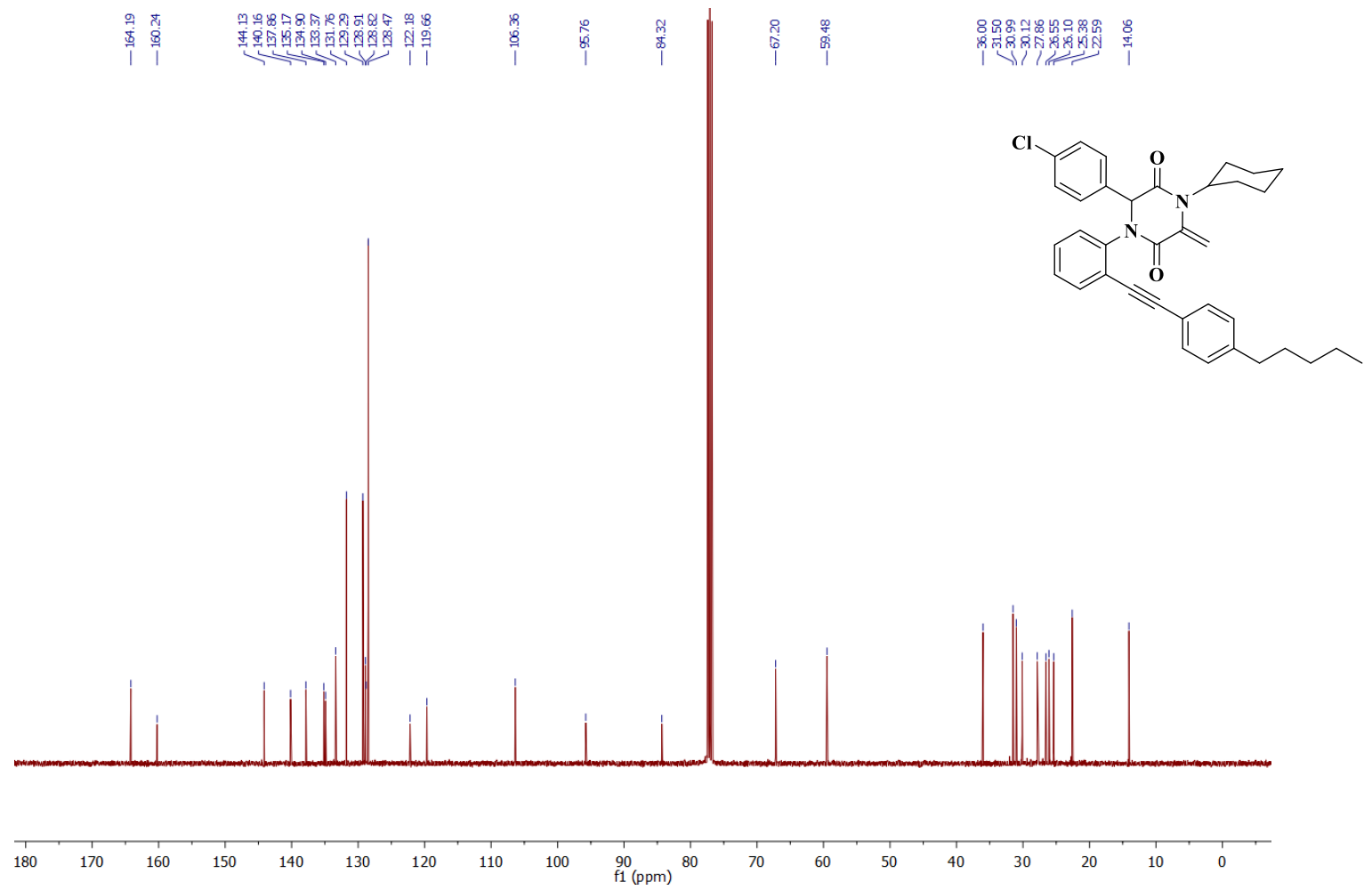

Figure S20. ${ }^{13} \mathrm{C}$ NMR $\left(150 \mathrm{MHz}, \mathrm{CDCl}_{3}\right) \mathbf{1} \mathbf{j}$ 


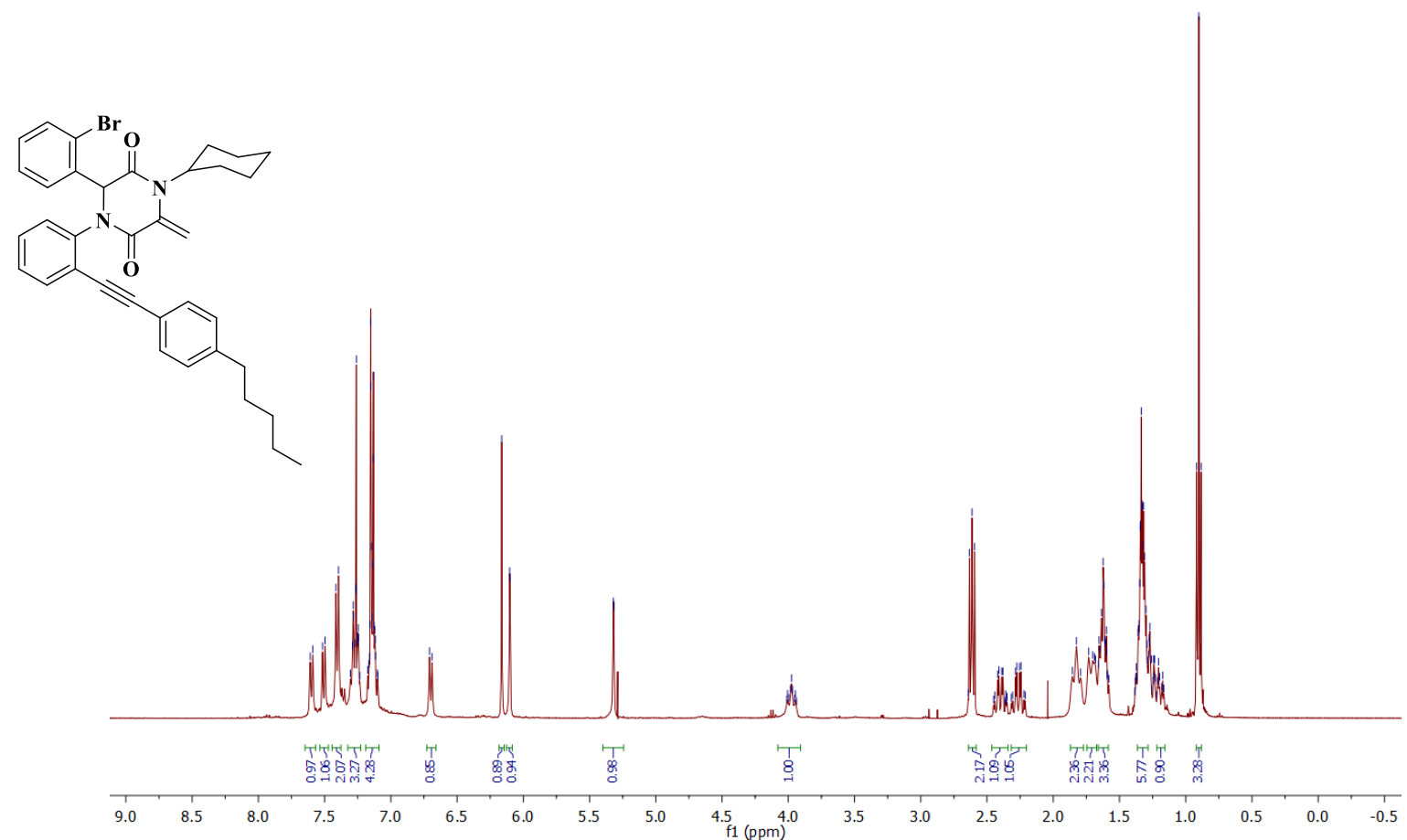

Figure S21. ${ }^{1} \mathrm{H}$ NMR $\left(400 \mathrm{MHz}, \mathrm{CDCl}_{3}\right) \mathbf{1 k}$
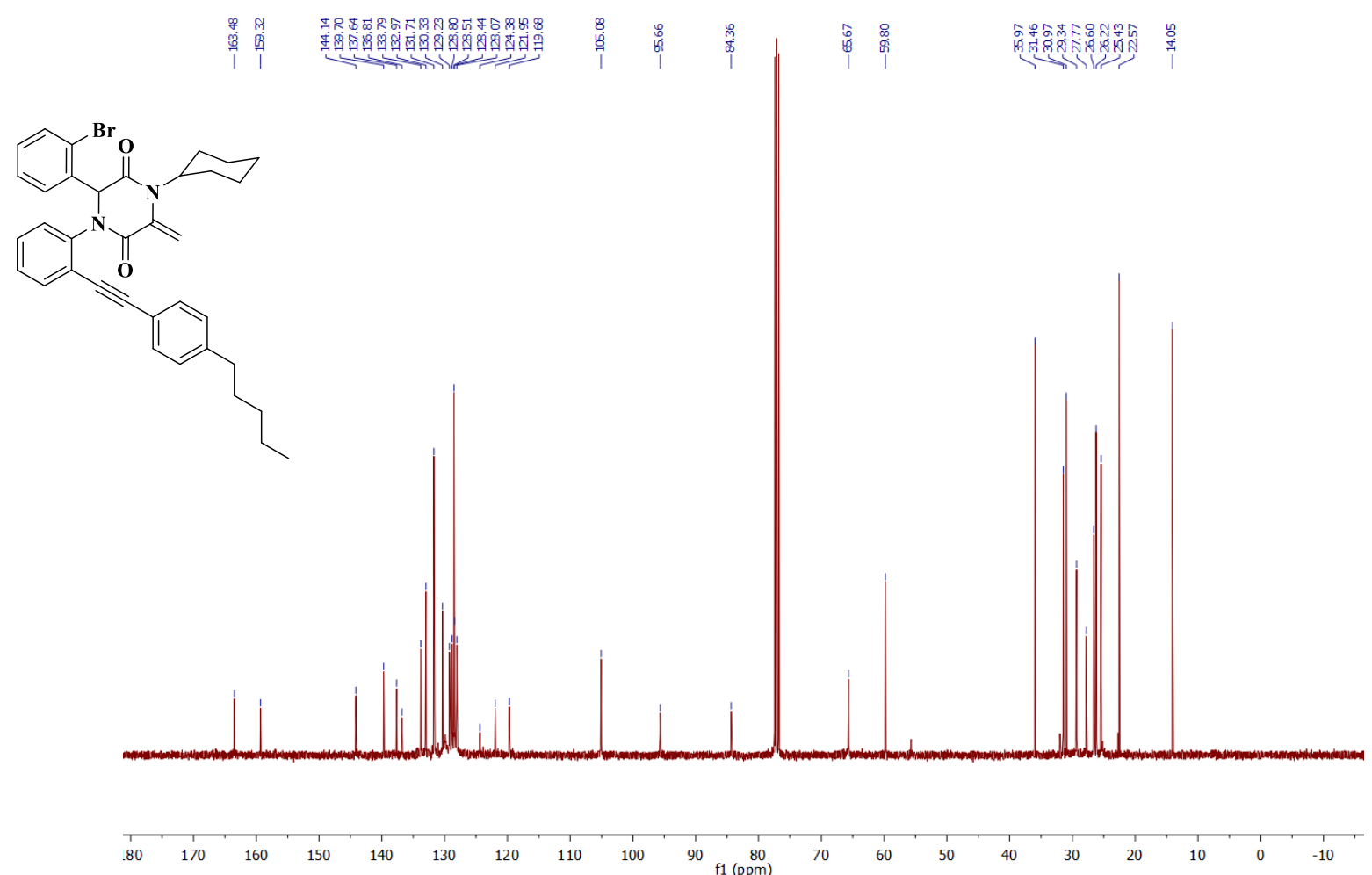

Figure S22. ${ }^{13} \mathrm{C}$ NMR(100 MHz, $\left.\mathrm{CDCl}_{3}\right) \mathbf{1 k}$ 


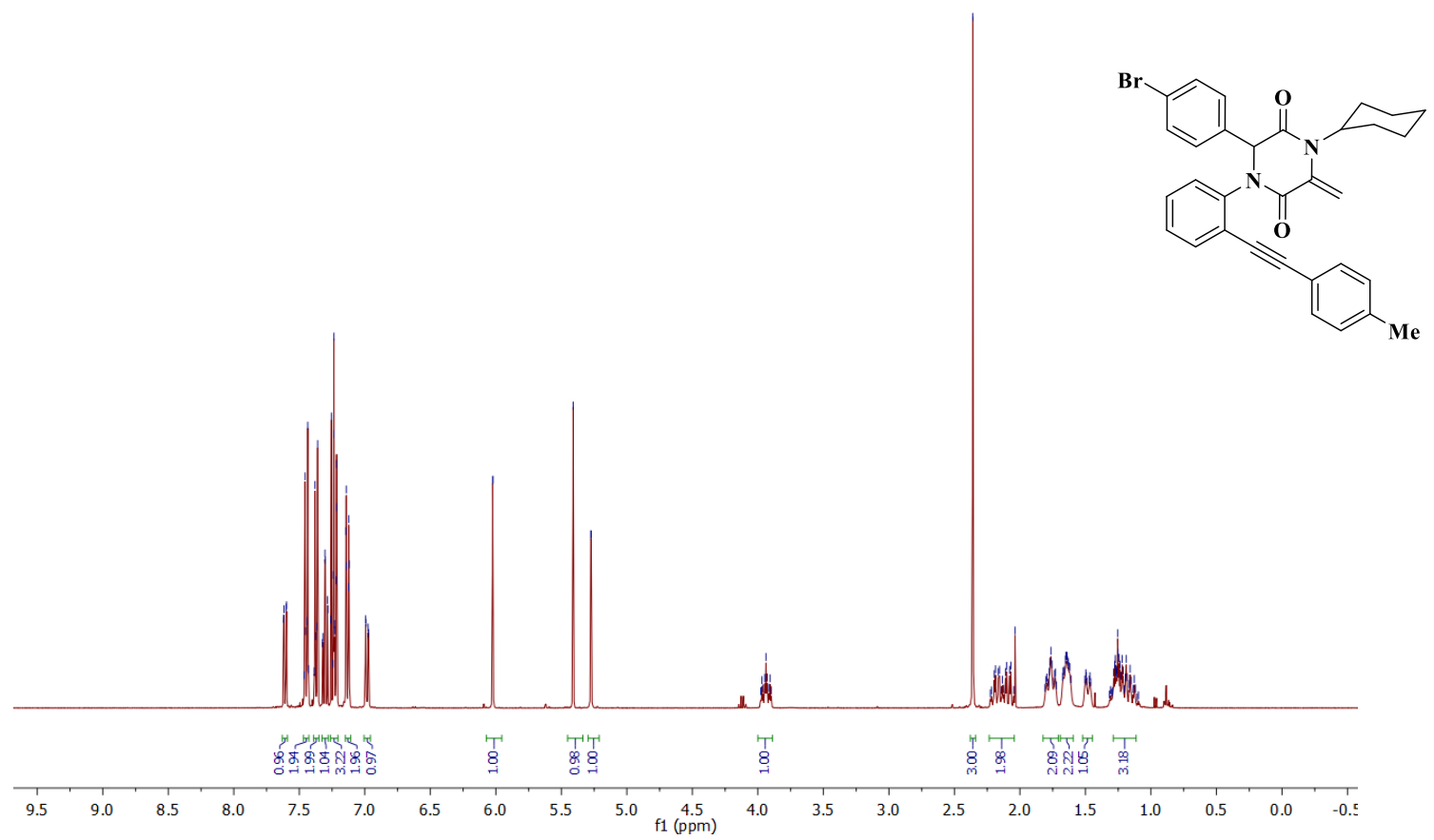

Figure S23. ${ }^{1} \mathrm{H} \mathrm{NMR}\left(400 \mathrm{MHz}, \mathrm{CDCl}_{3}\right) \mathbf{1 l}$

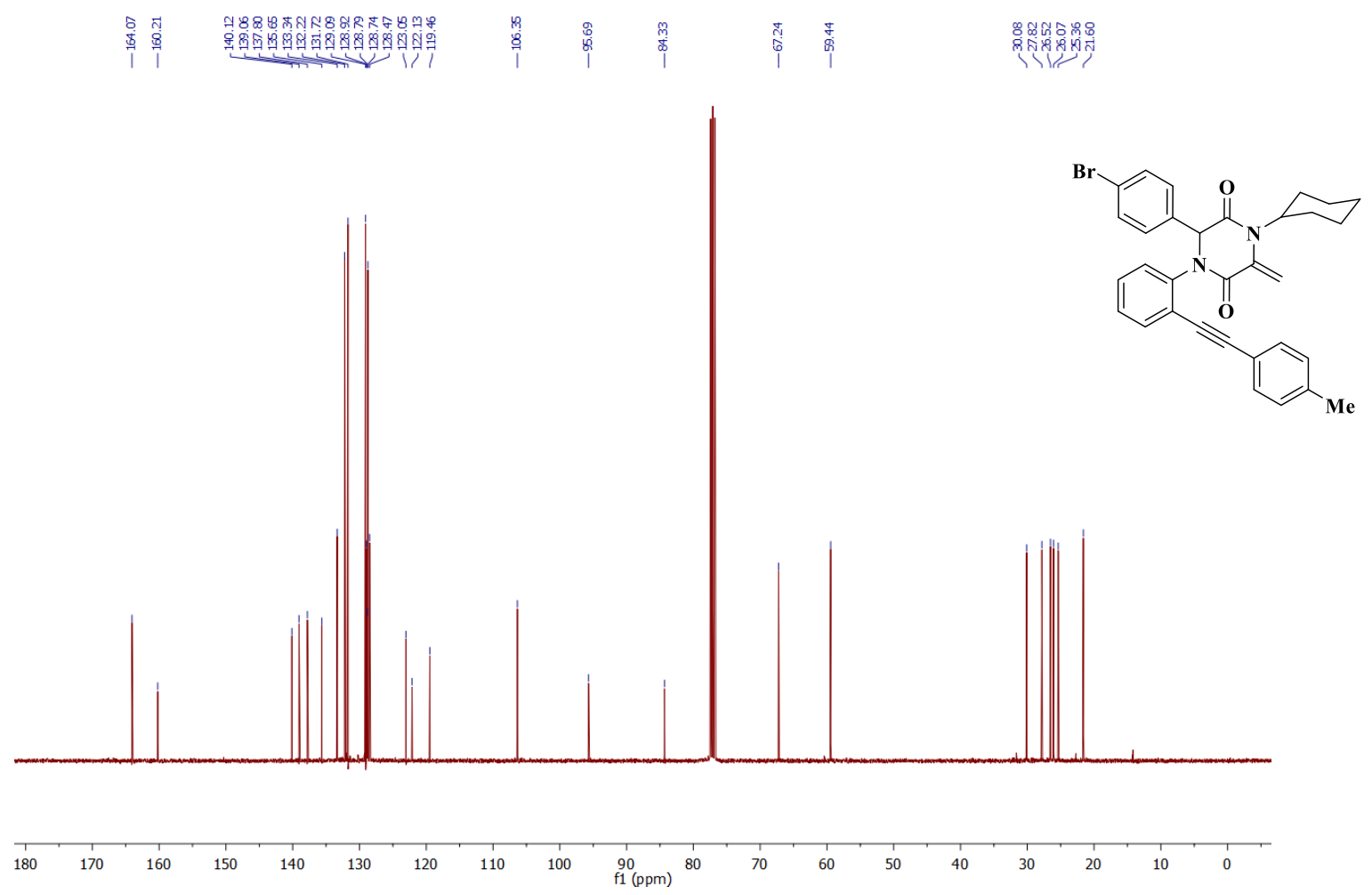

Figure S24. ${ }^{13} \mathrm{C}$ NMR $\left(100 \mathrm{MHz}, \mathrm{CDCl}_{3}\right) 1 \mathrm{I}$ 


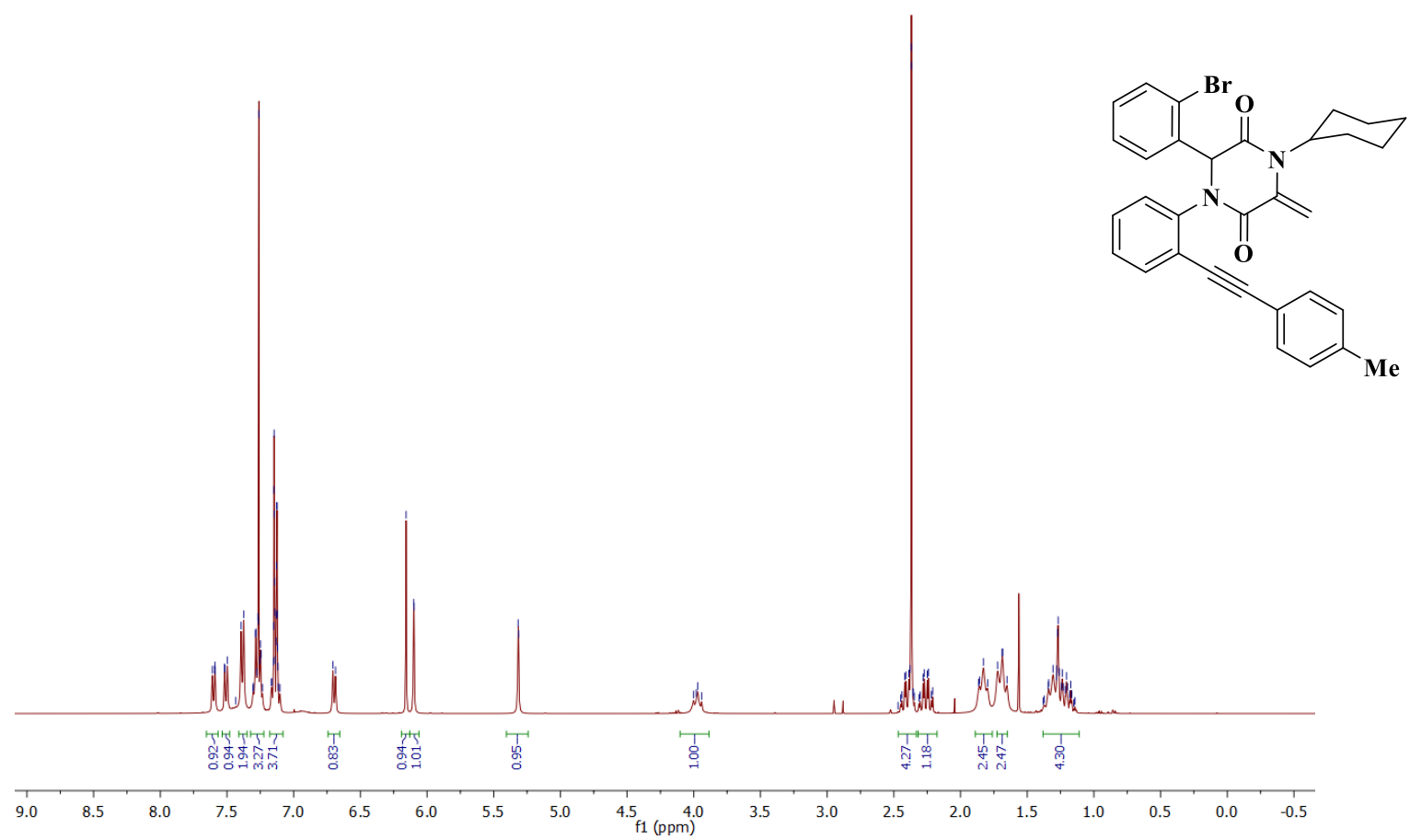

Figure S25. ${ }^{1} \mathrm{H} \mathrm{NMR}\left(400 \mathrm{MHz}, \mathrm{CDCl}_{3}\right) \mathbf{1 m}$

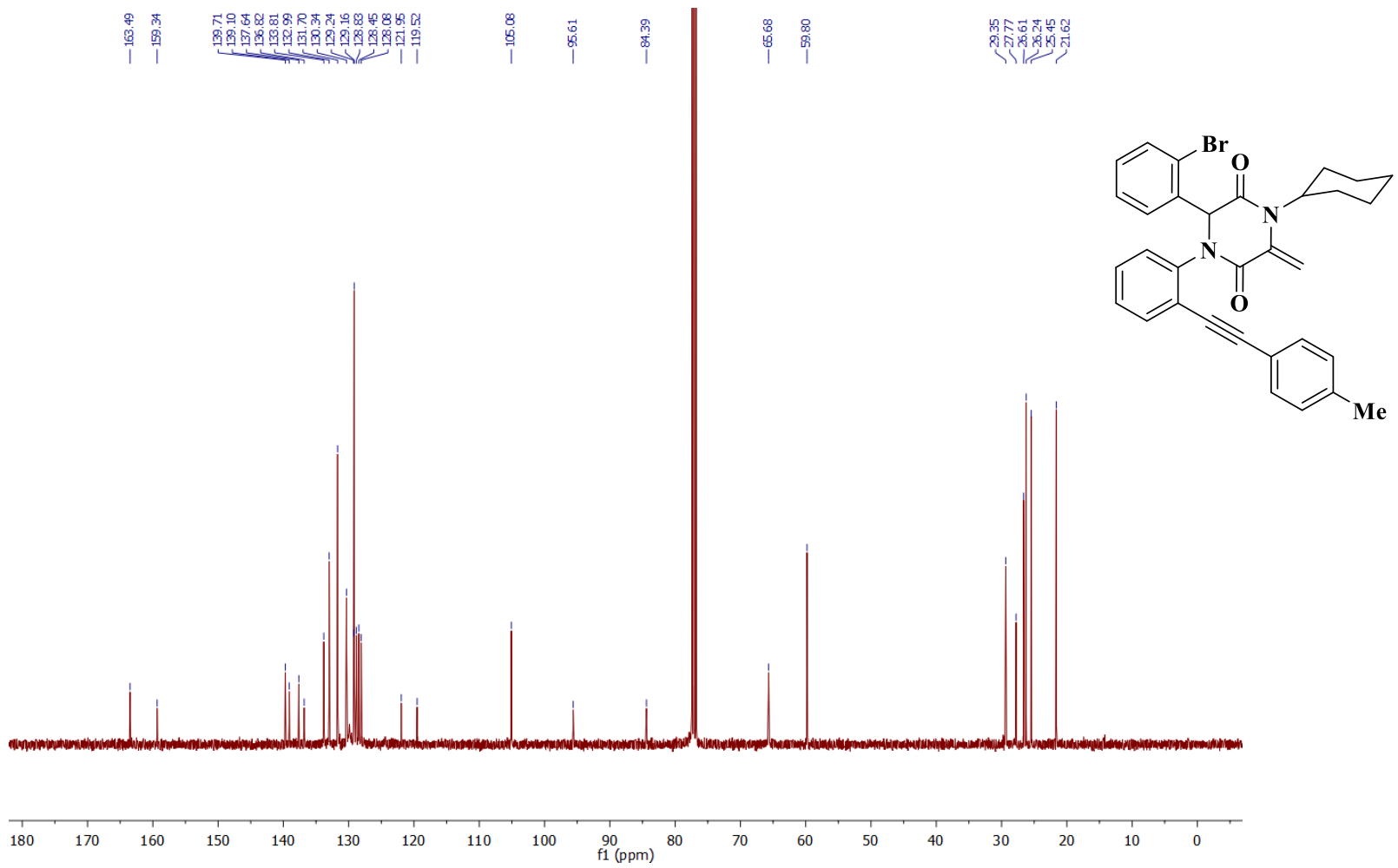

Figure S26. ${ }^{13} \mathrm{C} \mathrm{NMR}\left(100 \mathrm{MHz}, \mathrm{CDCl}_{3}\right) \mathbf{1 m}$ 


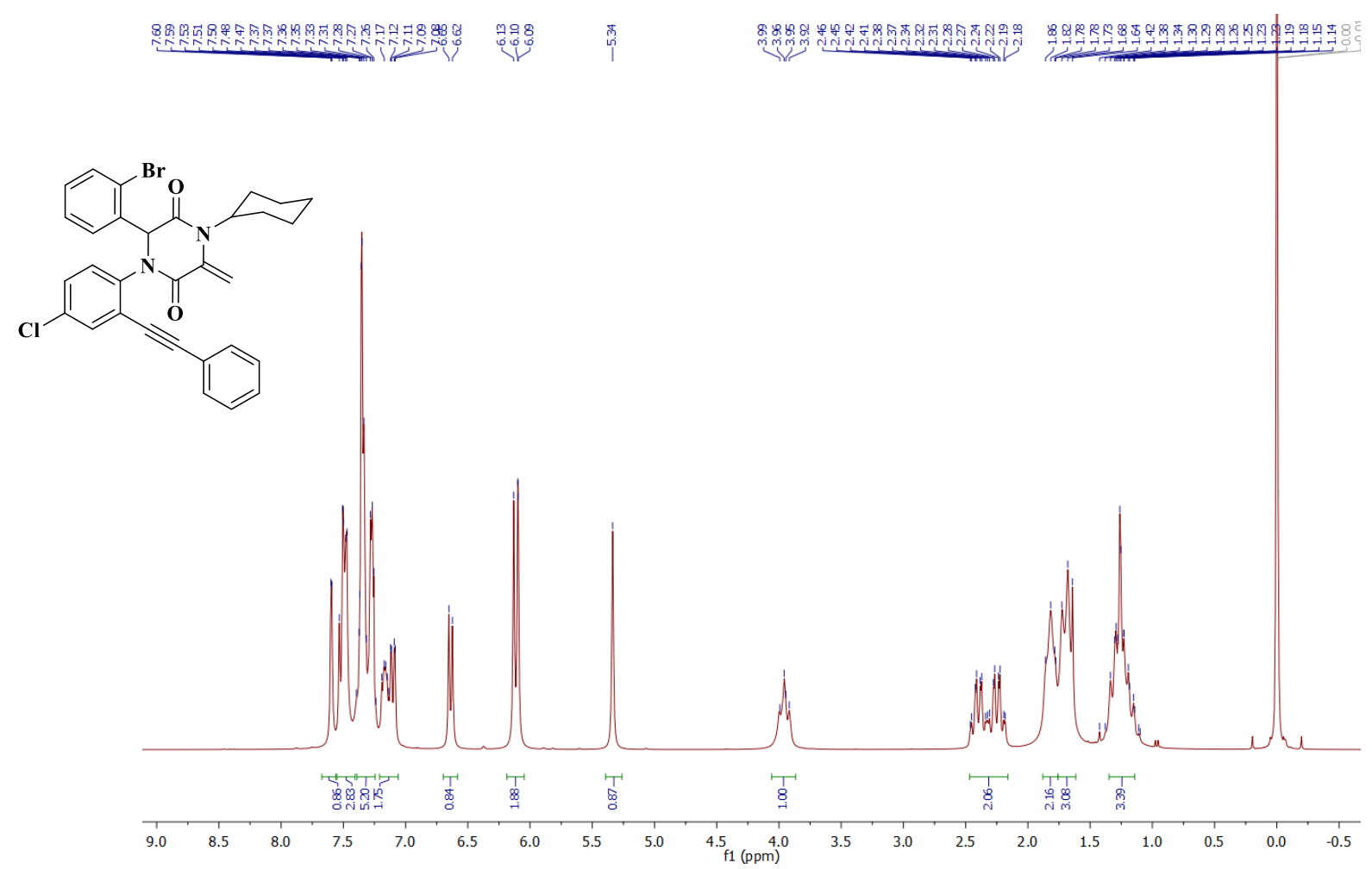

Figure S27. ${ }^{1} \mathrm{H}$ NMR(300 MHz, $\left.\mathrm{CDCl}_{3}\right)$ 1n

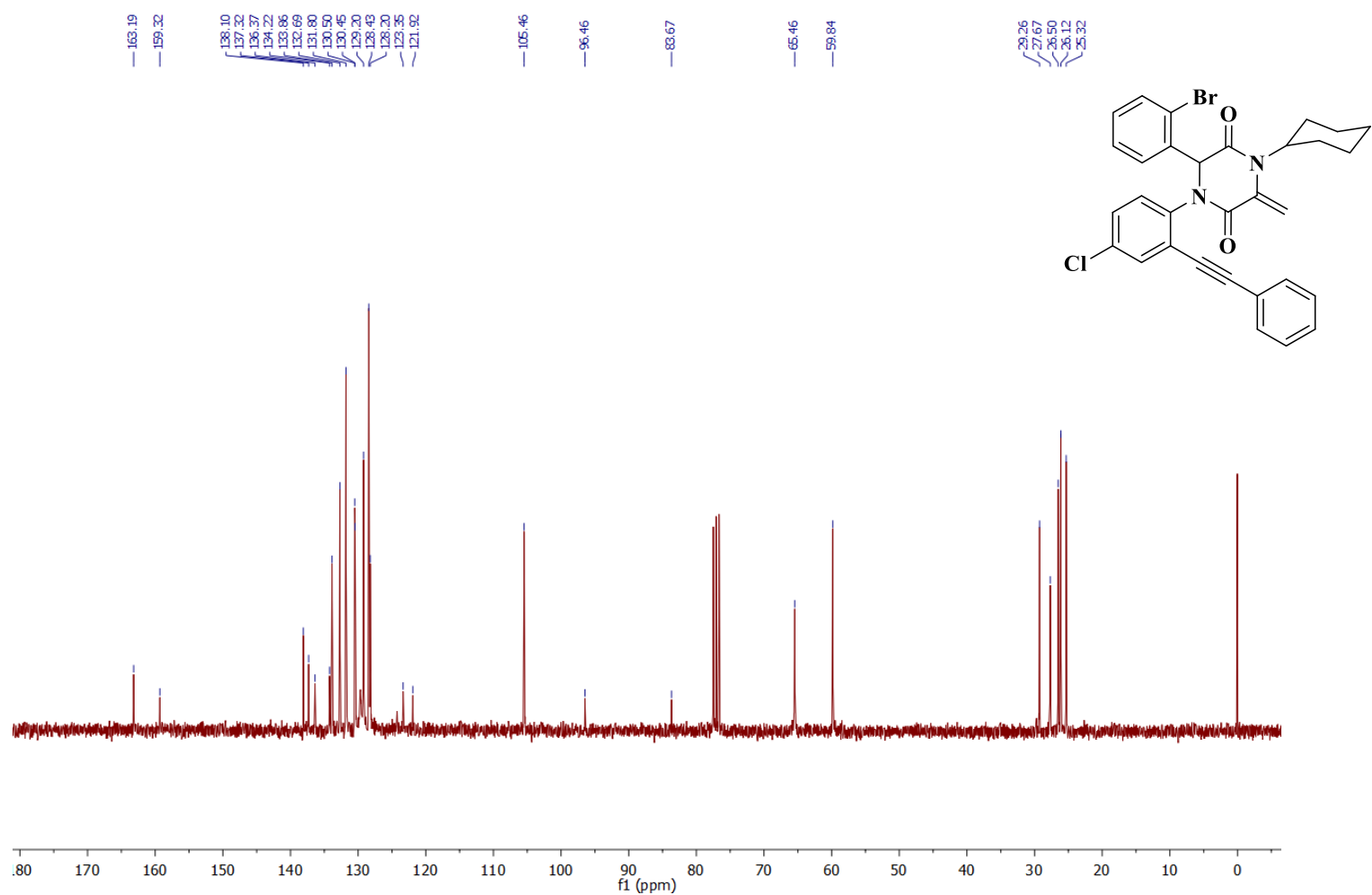

Figure S28. ${ }^{13} \mathrm{C}$ NMR(75 MHz, $\left.\mathrm{CDCl}_{3}\right)$ 1n 


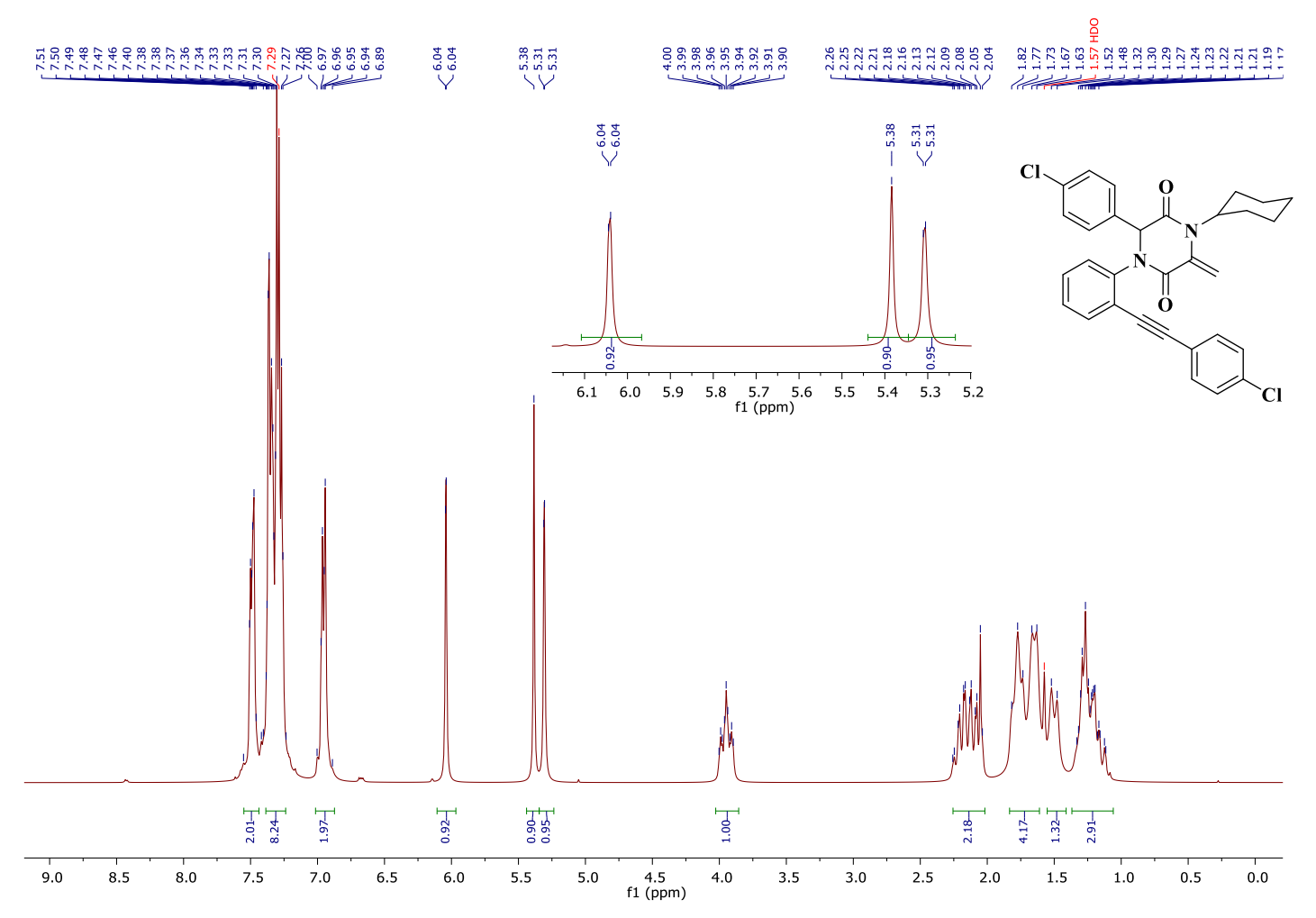

Figure S29. ${ }^{1} \mathrm{H} \mathrm{NMR}\left(300 \mathrm{MHz}, \mathrm{CDCl}_{3}\right) \mathbf{1 p}$

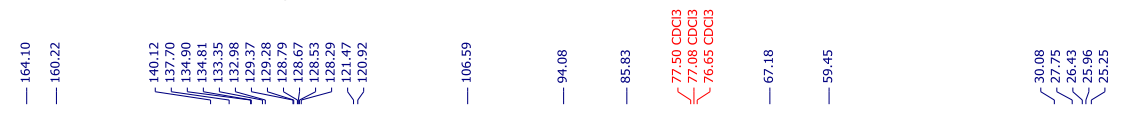

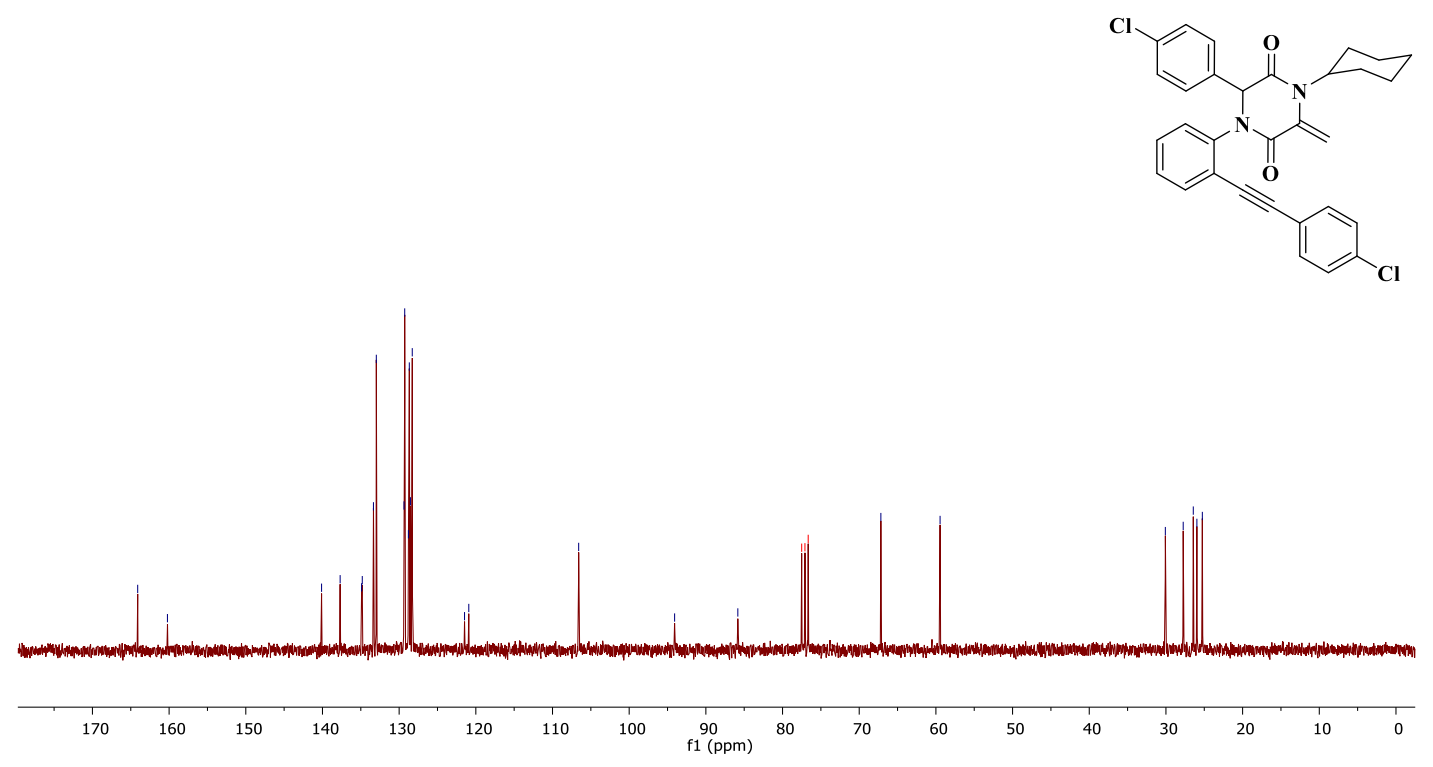

Figure $\mathrm{S} 30 .{ }^{13} \mathrm{C}$ NMR( $\left.75 \mathrm{MHz}, \mathrm{CDCl}_{3}\right) \mathbf{1 p}$ 


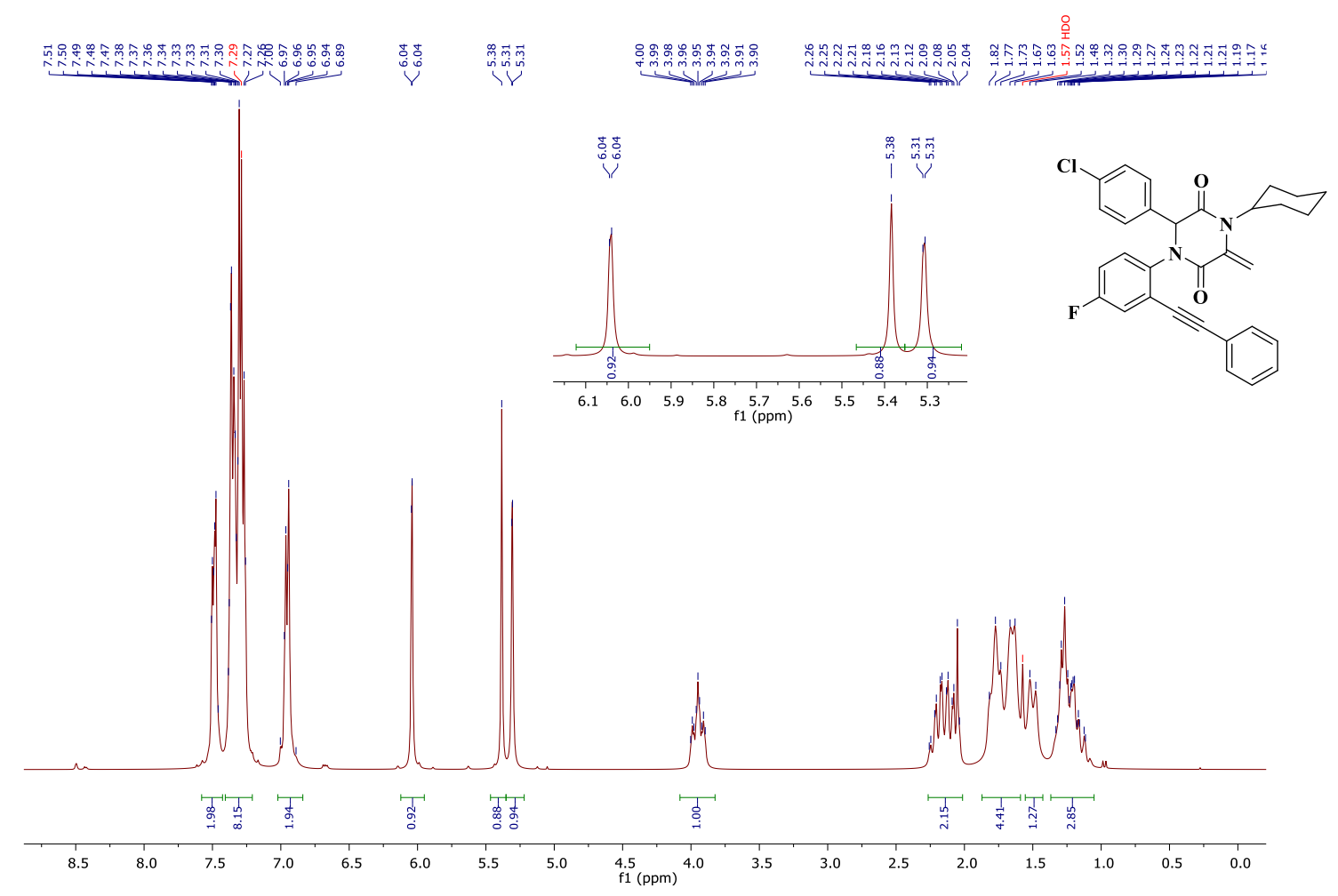

Figure $\mathrm{S} 31 .{ }^{1} \mathrm{H}$ NMR(300 MHz, $\left.\mathrm{CDCl}_{3}\right) 1$ o

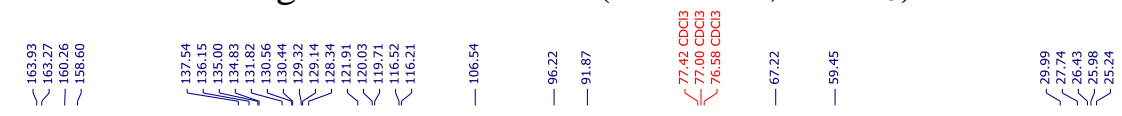

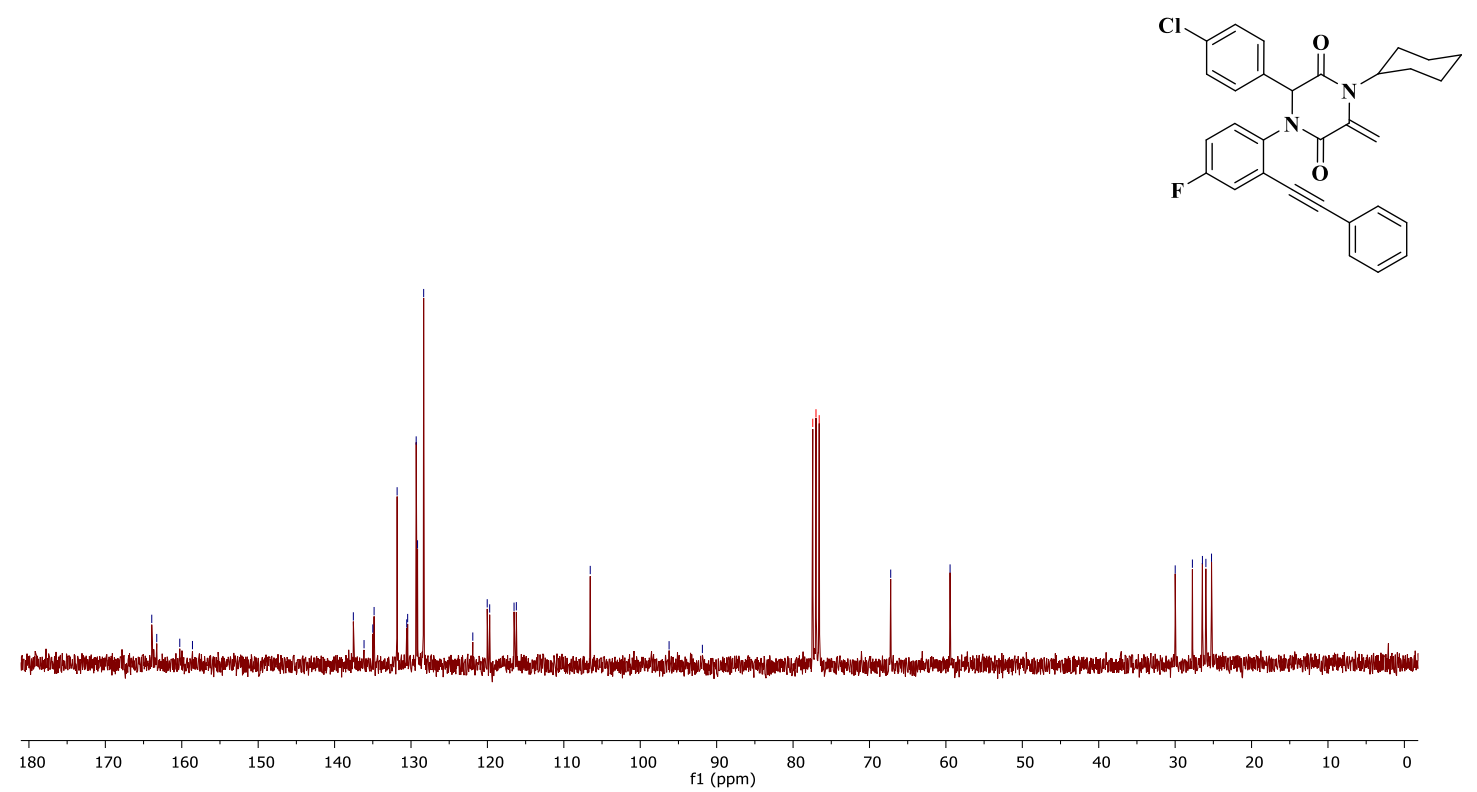

Figure $\mathrm{S} 32 .{ }^{13} \mathrm{C} \mathrm{NMR}\left(75 \mathrm{MHz}, \mathrm{CDCl}_{3}\right) \mathbf{1 o}$ 


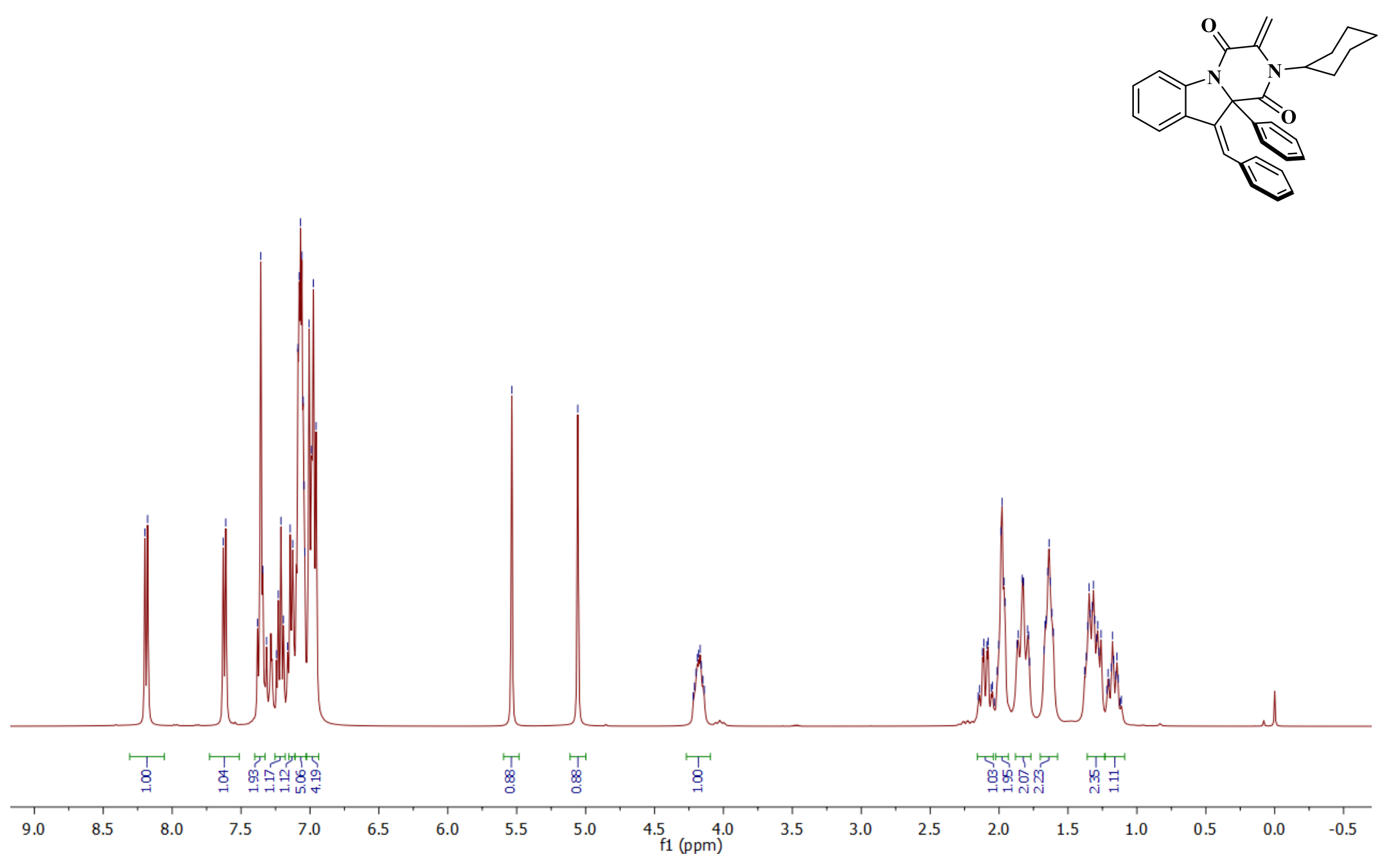

Figure $\mathrm{S} 33 .{ }^{1} \mathrm{H} \mathrm{NMR}\left(400 \mathrm{MHz}, \mathrm{CDCl}_{3}\right) \mathbf{2 a}$ 

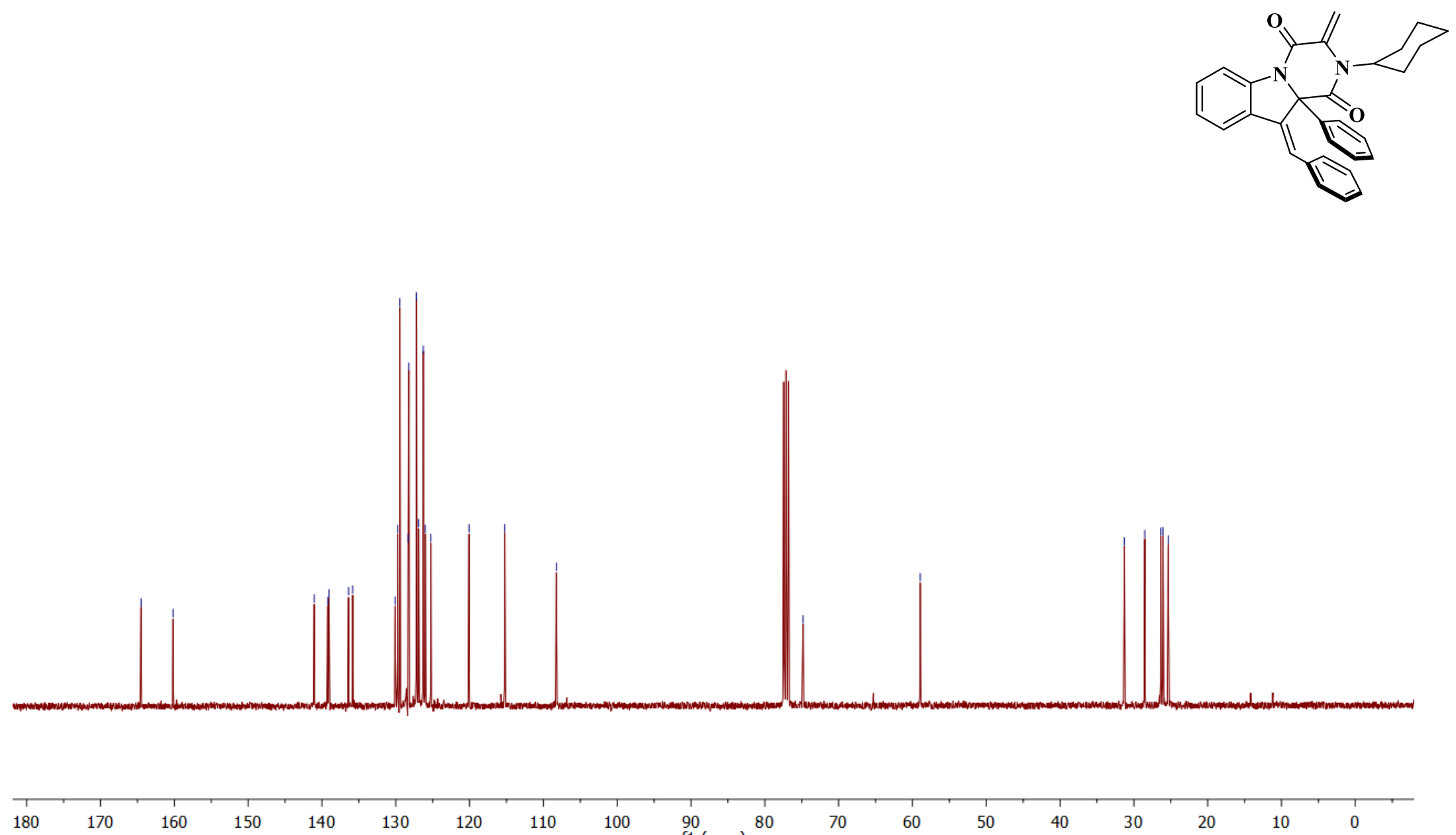

Figure $\mathrm{S} 34 .{ }^{13} \mathrm{C} \mathrm{NMR}\left(100 \mathrm{MHz}, \mathrm{CDCl}_{3}\right)$ 2a 
Dlota 158 hro1 :1 RT: 0.02 AV:1 $N: 2.03 E\}$

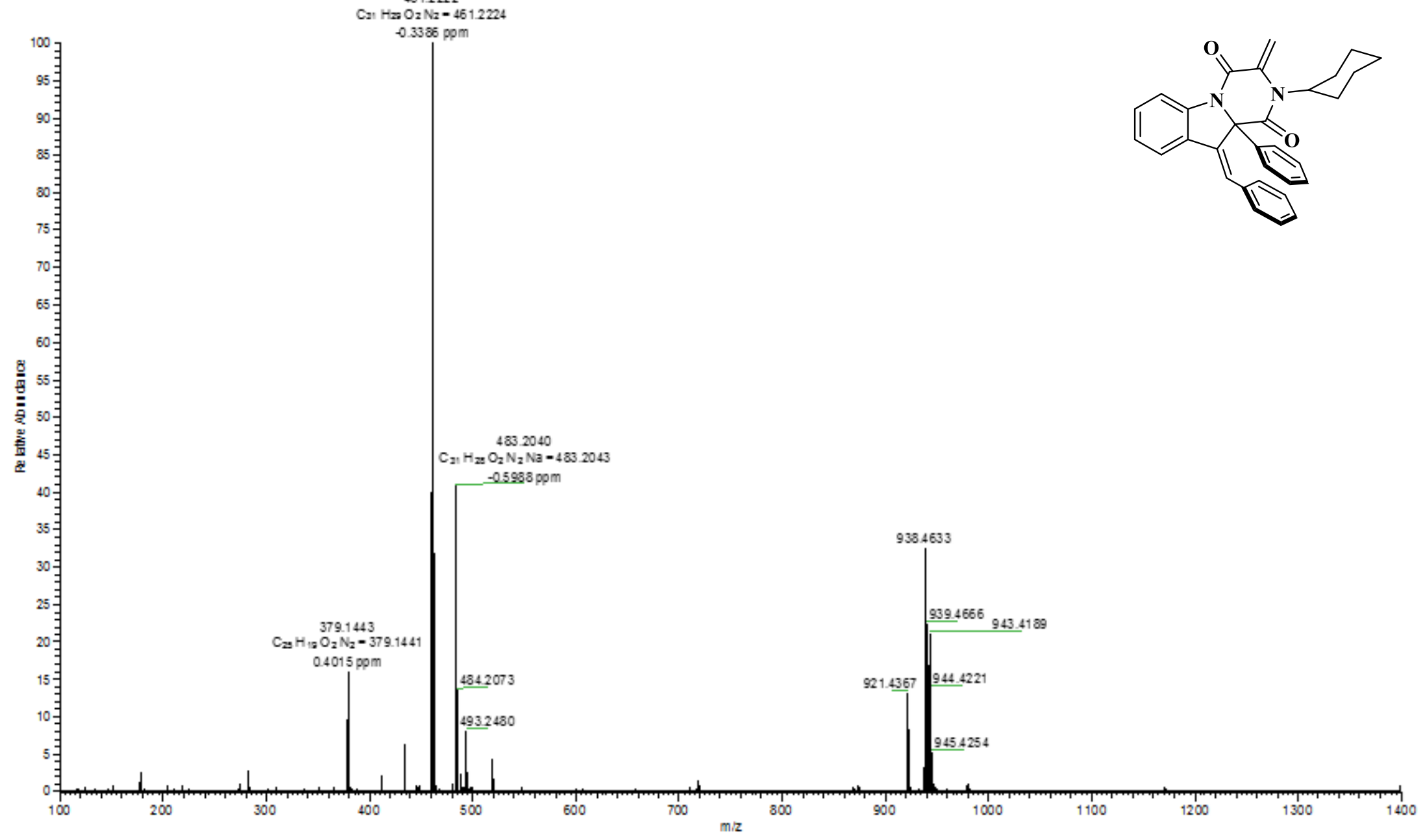

Figure S35. HRMS (ESI) 2a 

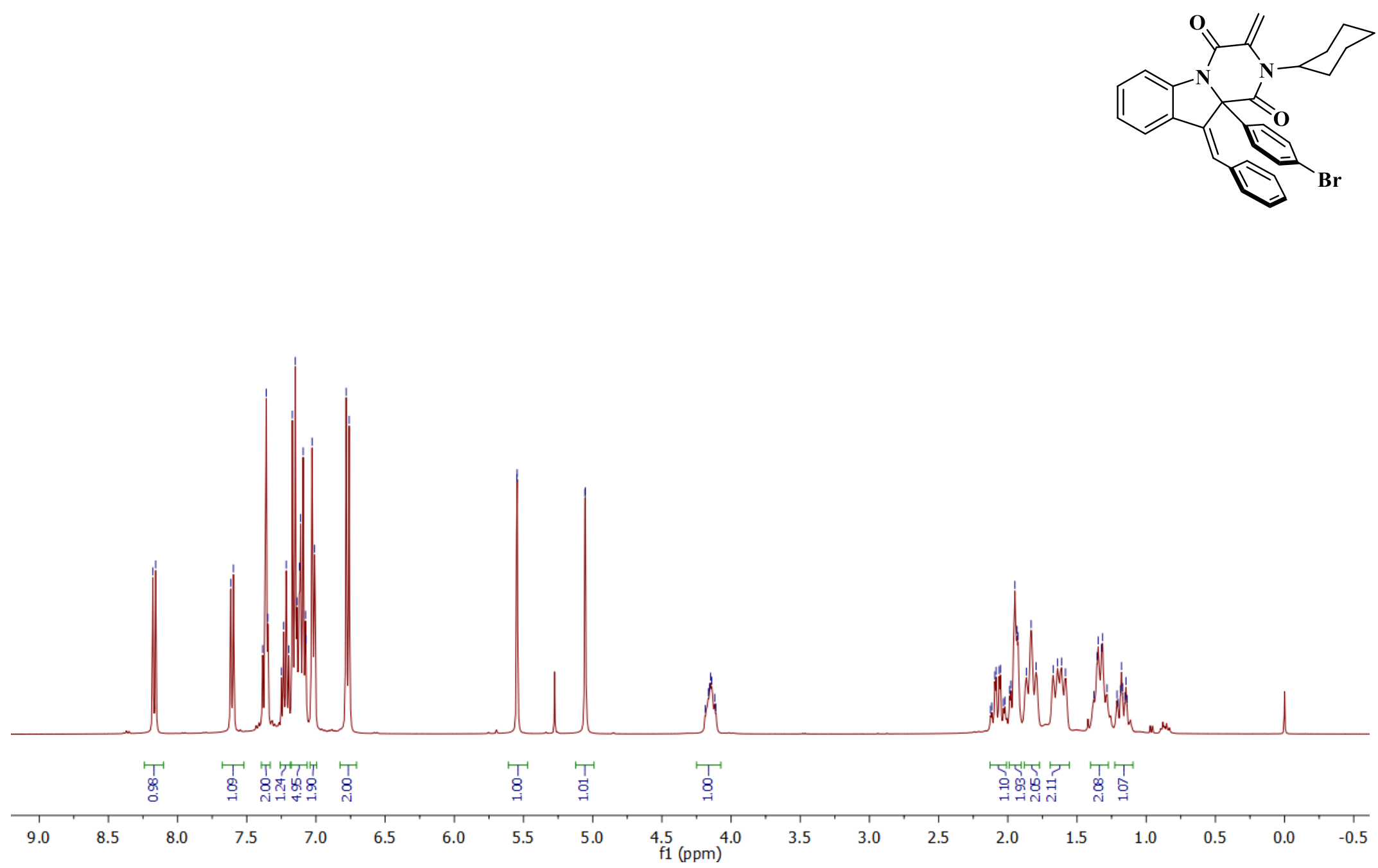

Figure $\mathrm{S} 36 .{ }^{1} \mathrm{H} \mathrm{NMR}\left(400 \mathrm{MHz}, \mathrm{CDCl}_{3}\right)$ 2b 

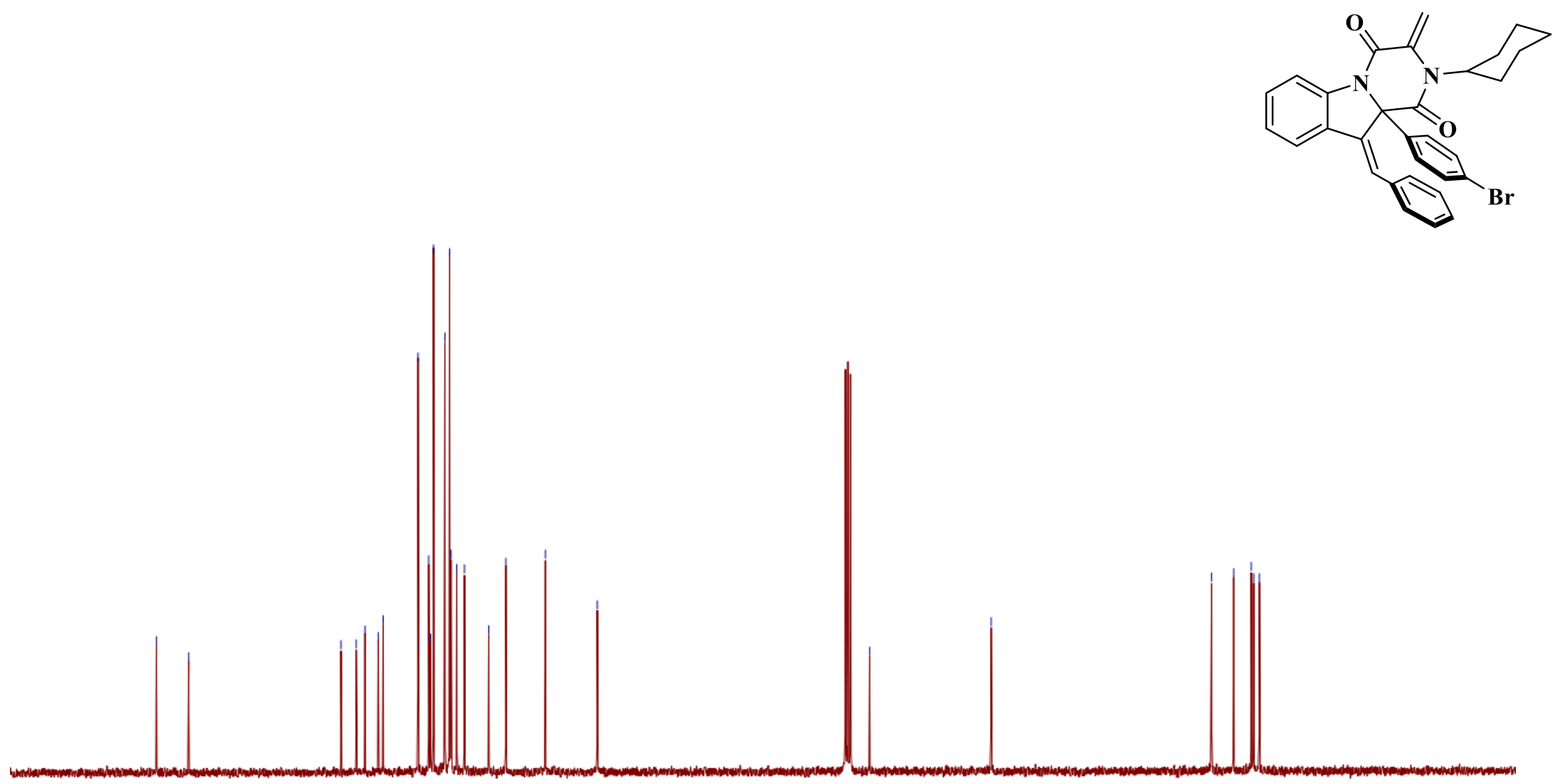

180 170 160 150 140 130 120 $110 \quad 100$ $\mathrm{f1}^{90} 80$
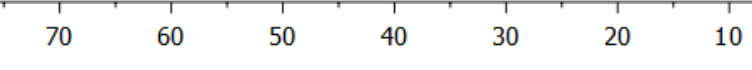

Figure S37. ${ }^{13} \mathrm{C} \mathrm{NMR}\left(100 \mathrm{MHz}, \mathrm{CDCl}_{3}\right) \mathbf{2 b}$ 
blota 136 nro1:1 RT:0.02 AV 1 N:4 33 E6

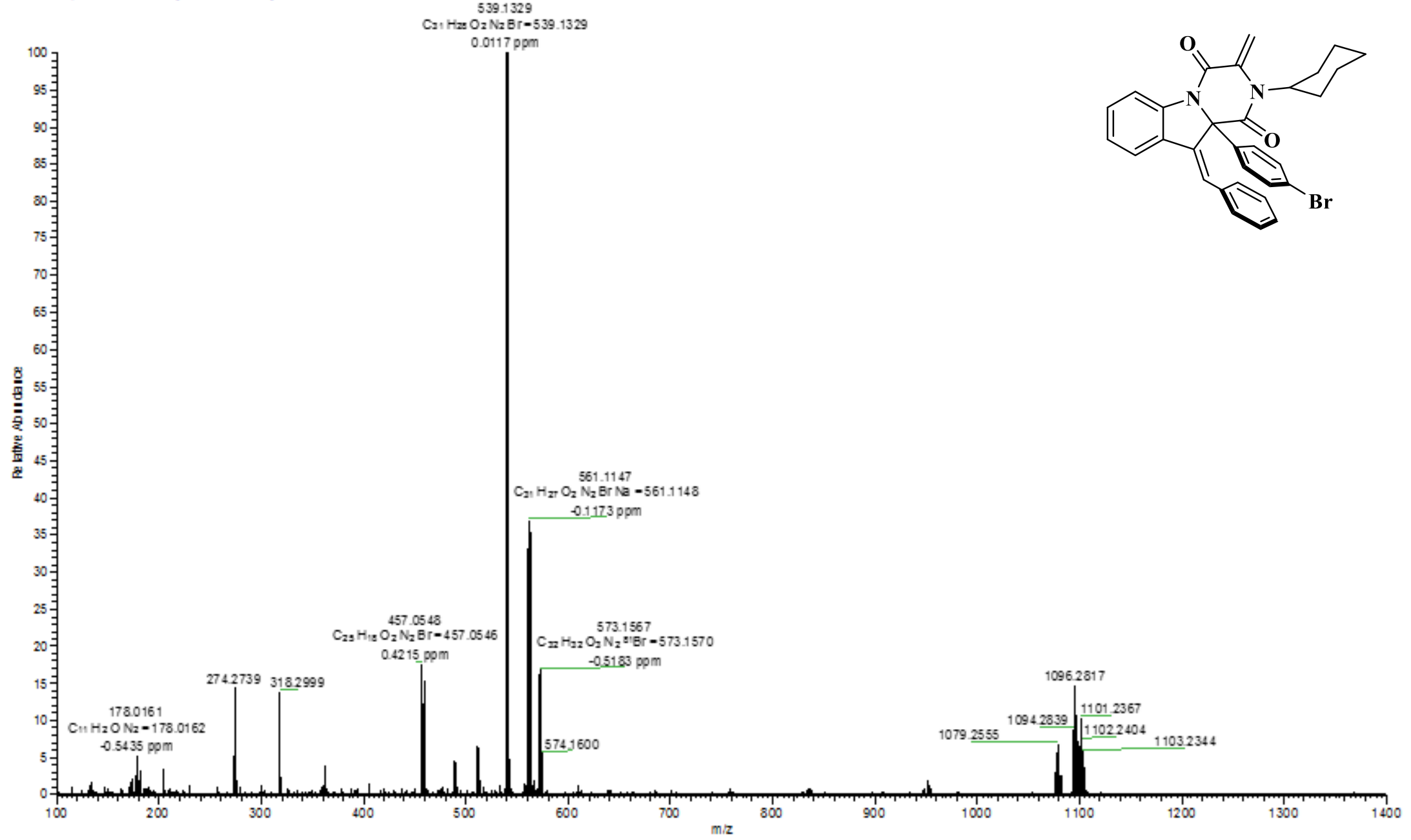

Figure S38. HRMS (ESI) 2b 

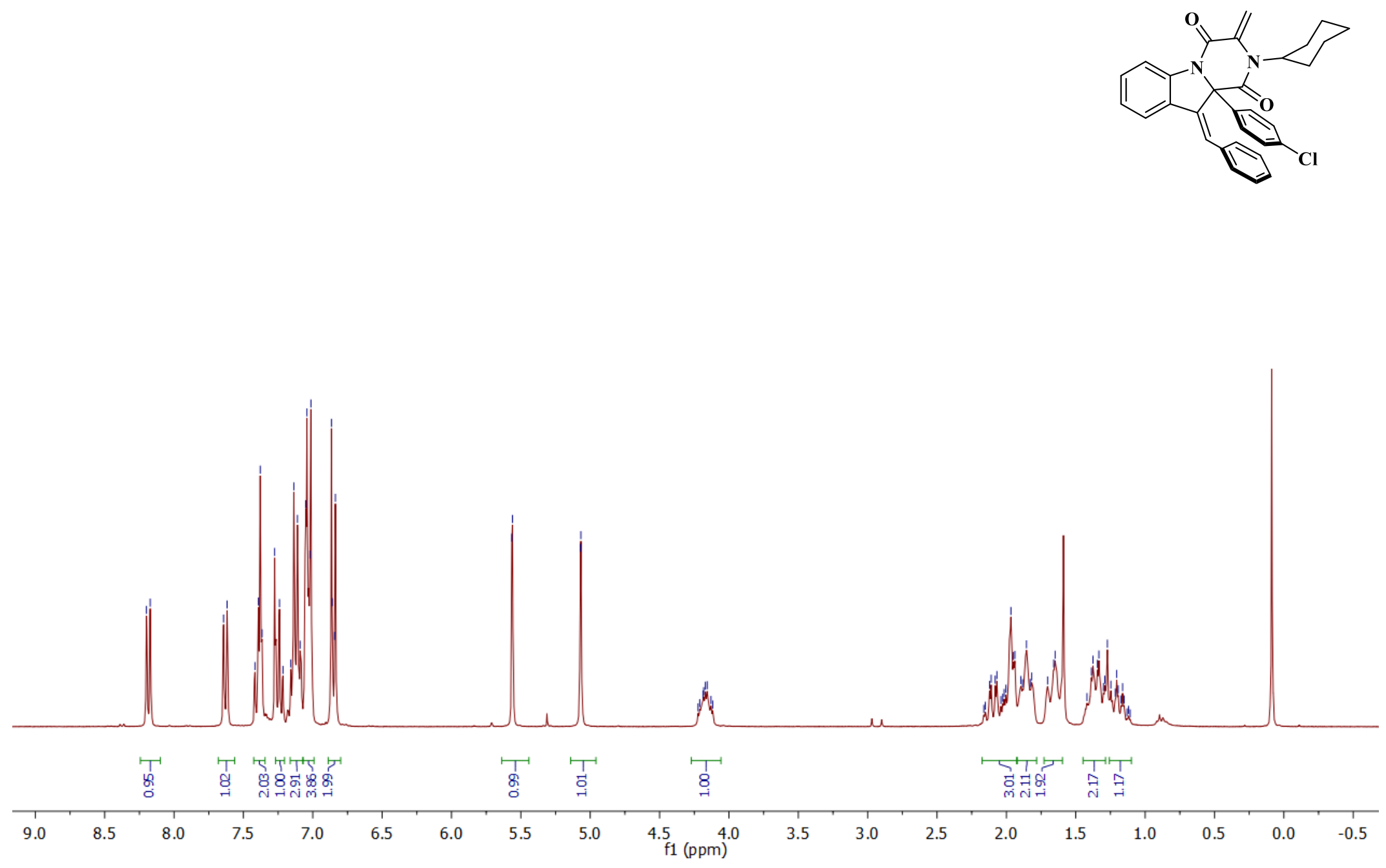

Figure S39. ${ }^{1} \mathrm{H} \mathrm{NMR}\left(300 \mathrm{MHz}, \mathrm{CDCl}_{3}\right)$ 2c 

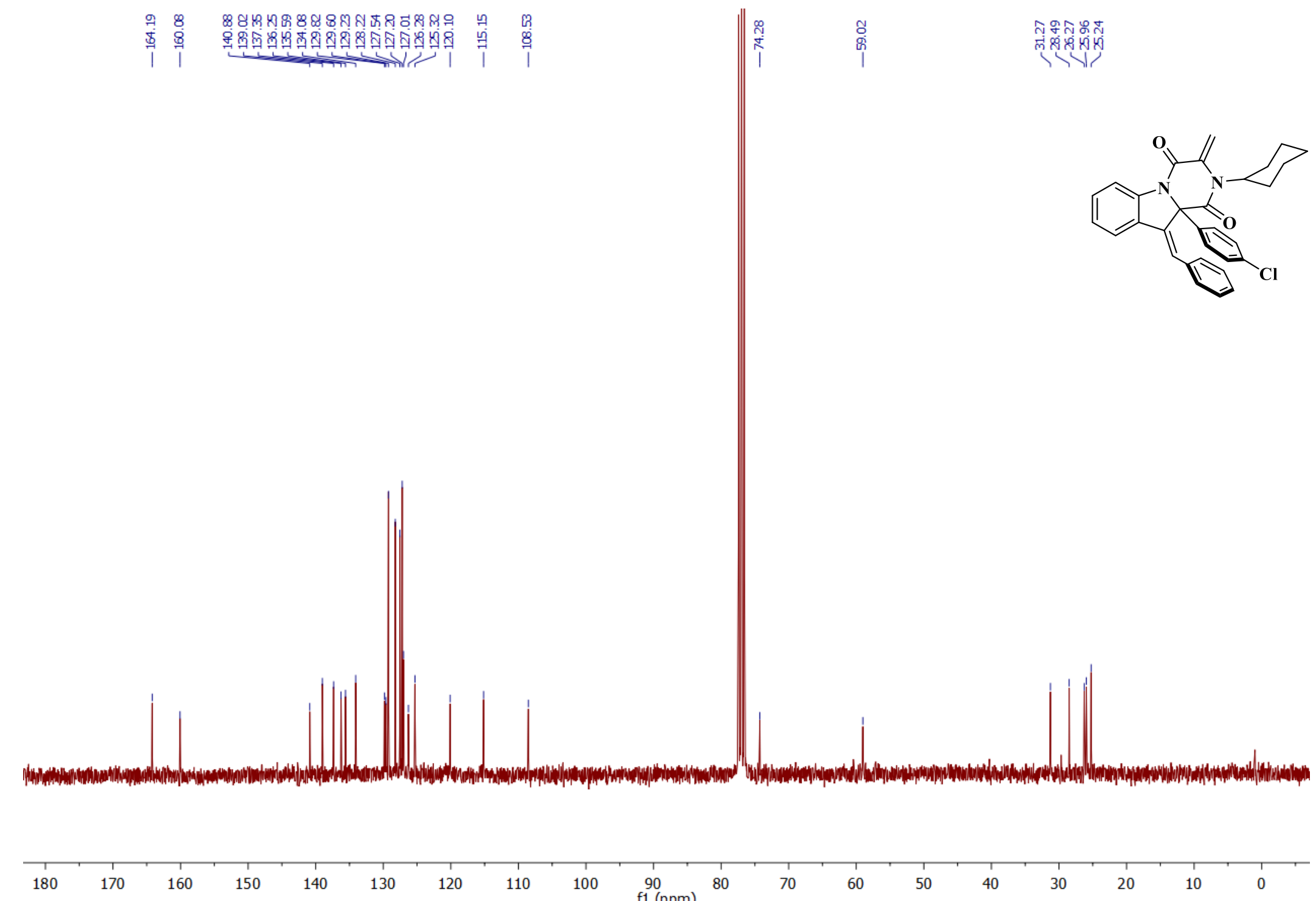

\begin{tabular}{|c|c|c|}
\hline 110 & 100 & $\begin{array}{c}90 \\
\mathrm{f} 1(\mathrm{ppm})\end{array}$ \\
\hline
\end{tabular}

$70 \quad 60$

50

Figure $\mathrm{S} 40 .{ }^{13} \mathrm{C} \mathrm{NMR}\left(75 \mathrm{MHz}, \mathrm{CDCl}_{3}\right) \mathbf{2 c}$ 


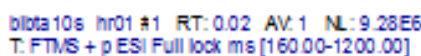

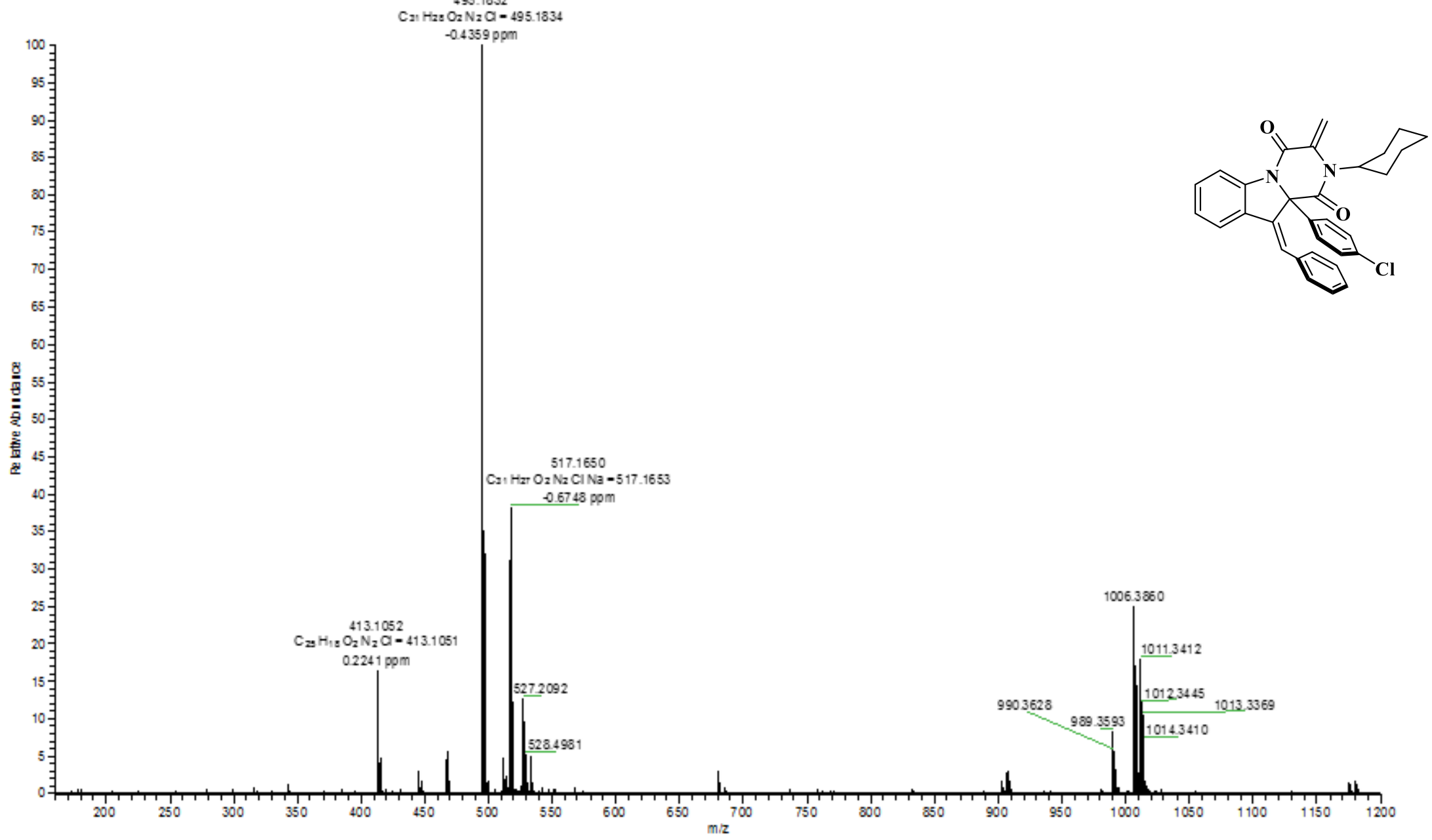

Figure S41. HRMS (ESI) 2c 

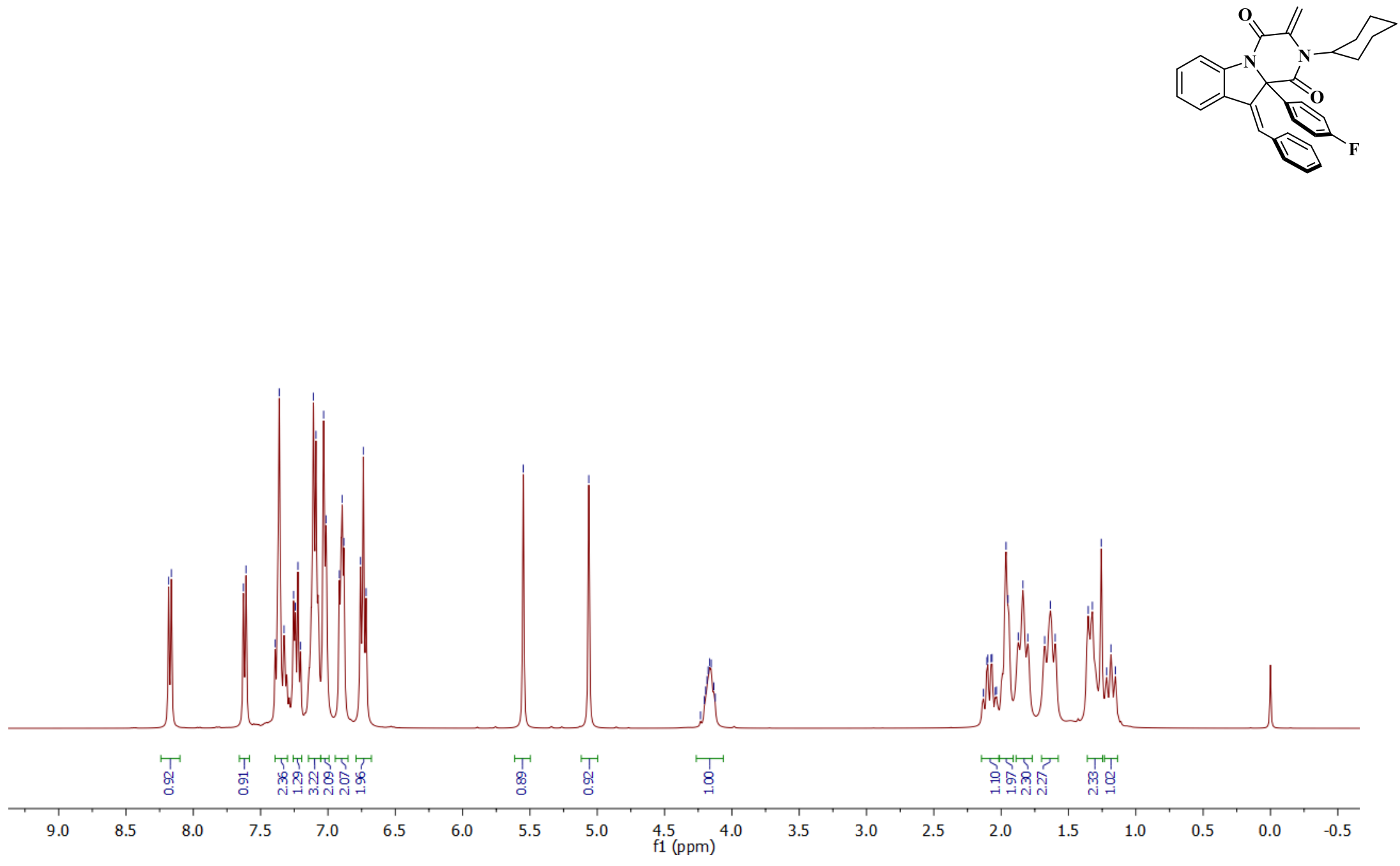

Figure $\mathrm{S} 42 .{ }^{1} \mathrm{H} \mathrm{NMR}\left(400 \mathrm{MHz}, \mathrm{CDCl}_{3}\right) \mathbf{2 d}$ 

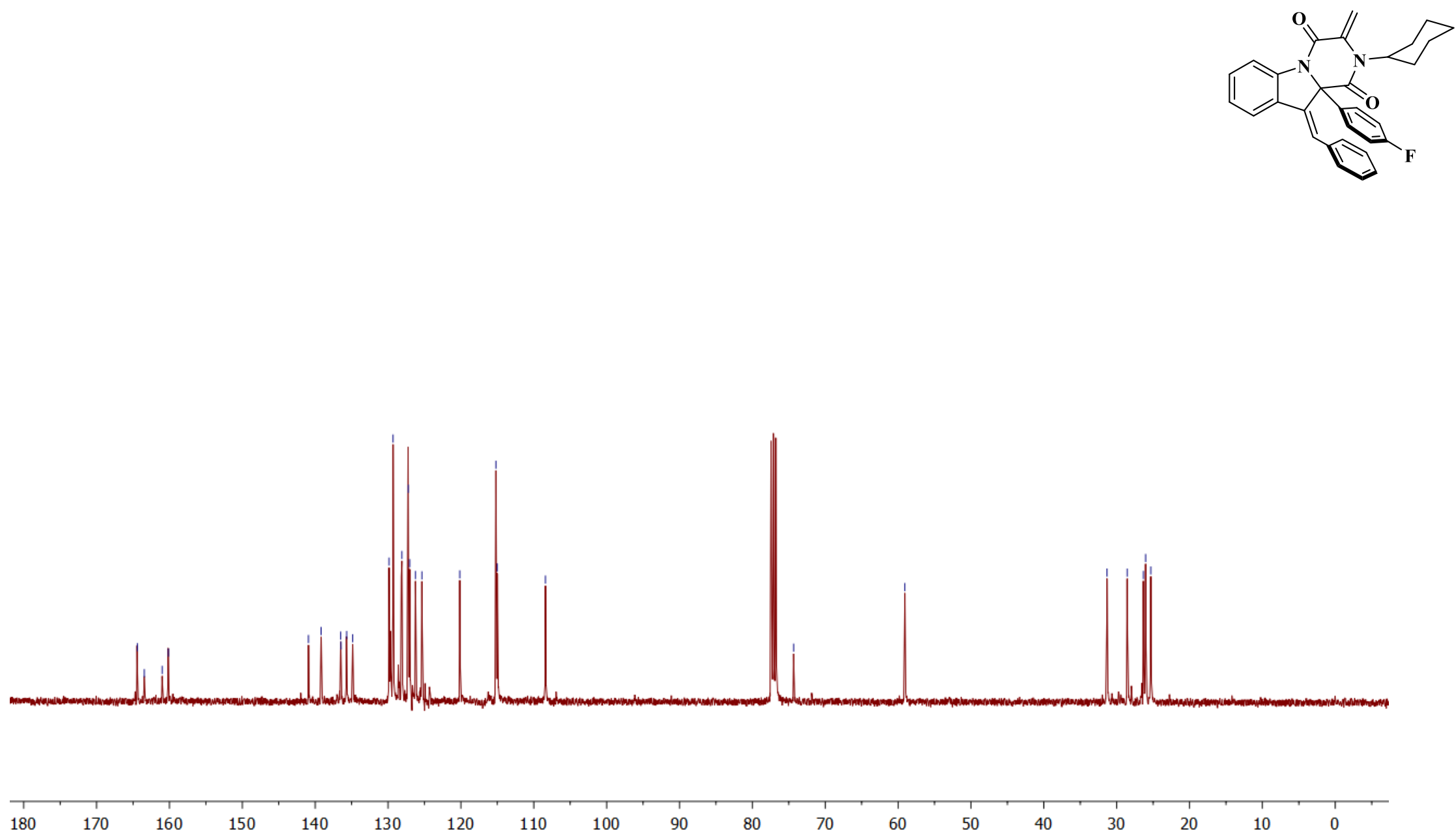

12
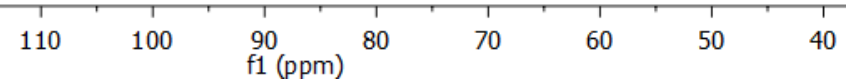

30

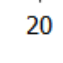

Figure $\mathrm{S} 43 .{ }^{13} \mathrm{C} \mathrm{NMR}\left(100 \mathrm{MHz}, \mathrm{CDCl}_{3}\right) \mathbf{2 d}$ 
Dlota 125 nro2 : 11 RT: 0.02 AV: 1 N $=4.5066$

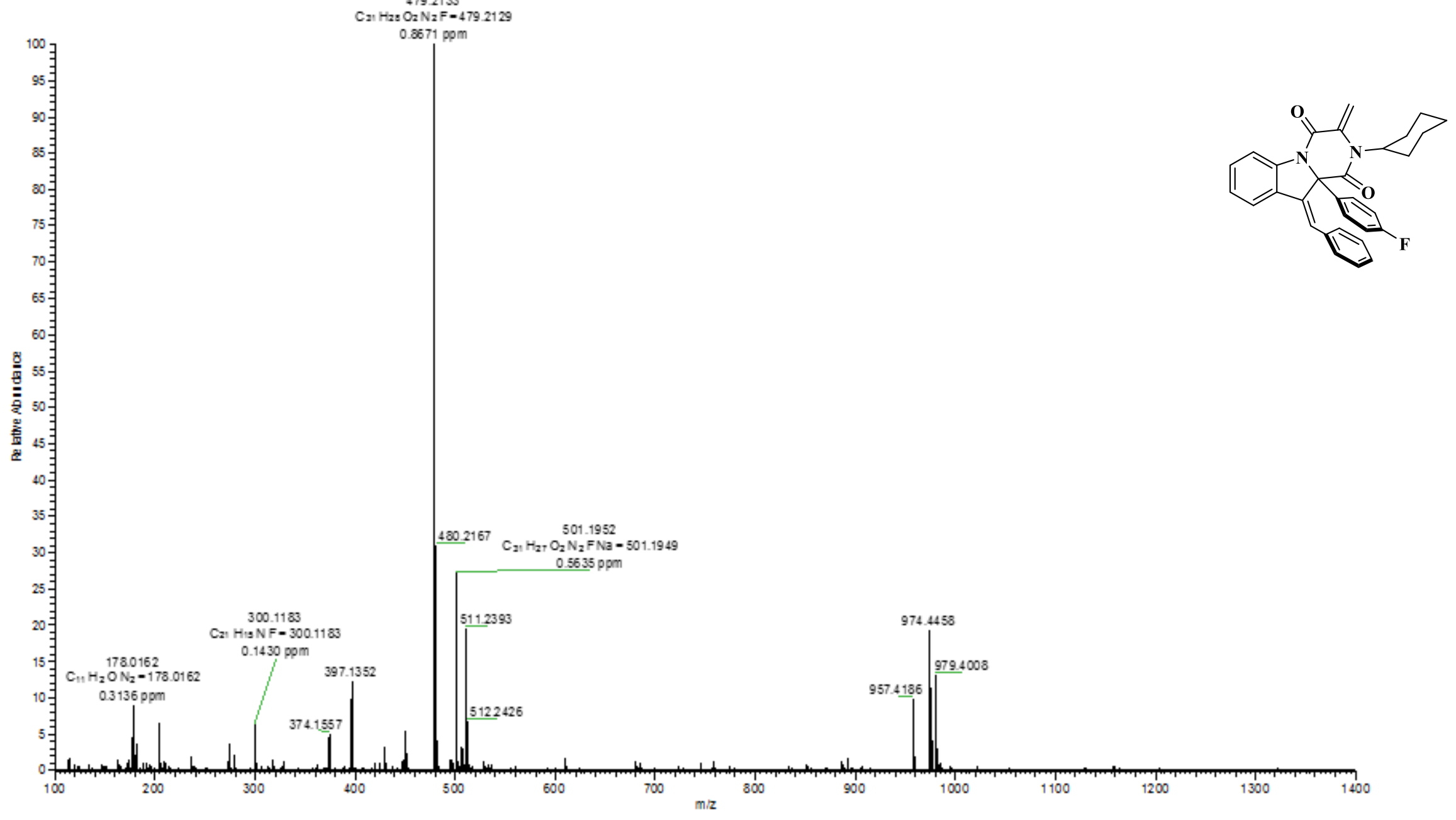

Figure S44. HRMS (ESI) 2d 


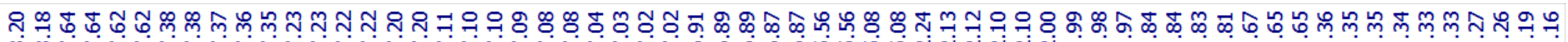

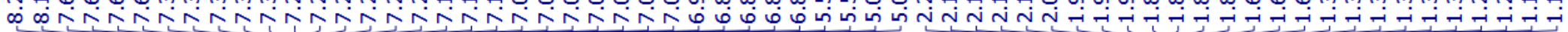

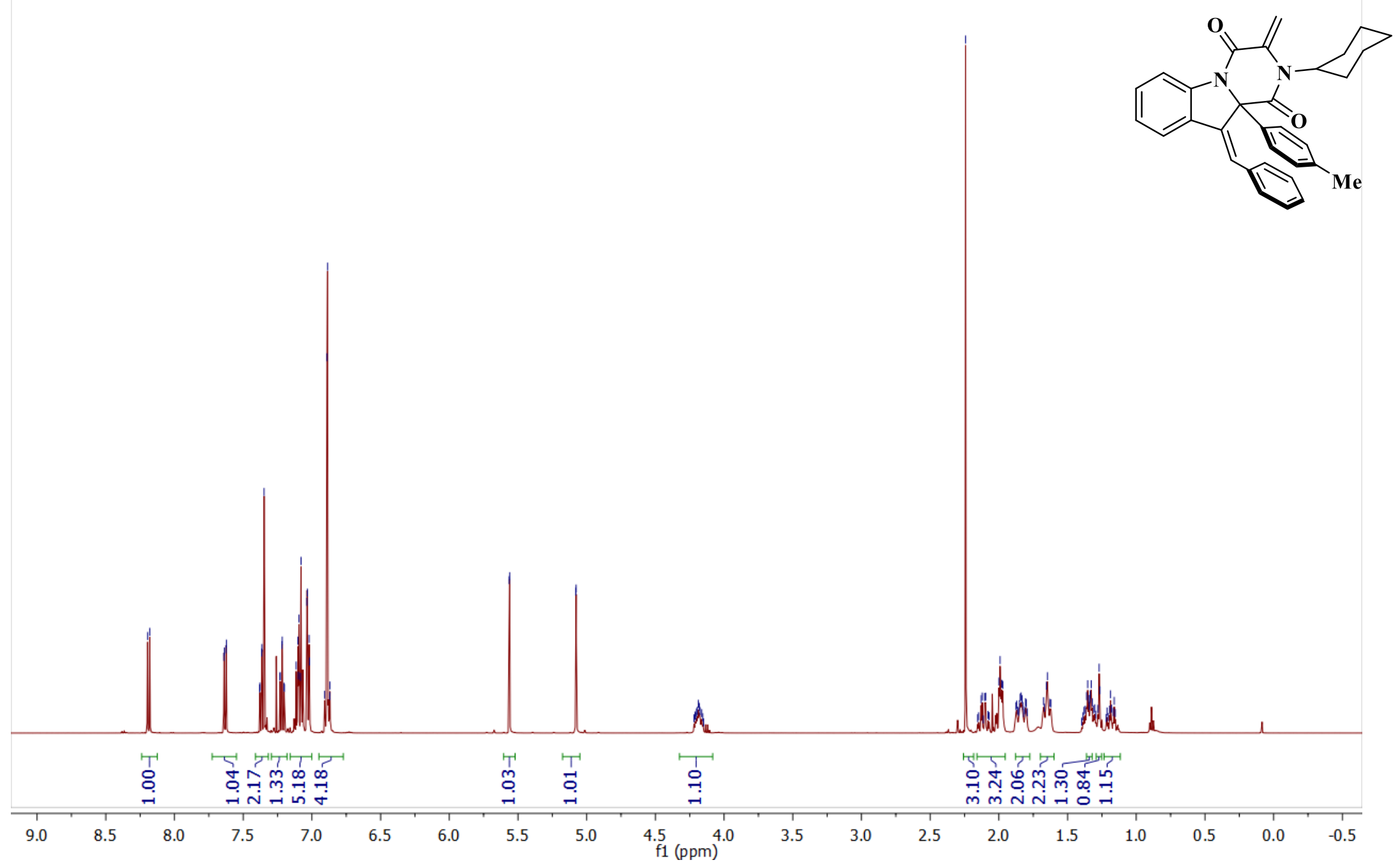

Figure $\mathrm{S} 45 .{ }^{1} \mathrm{H} \mathrm{NMR}\left(500 \mathrm{MHz}, \mathrm{CDCl}_{3}\right) \mathbf{2 e}$ 


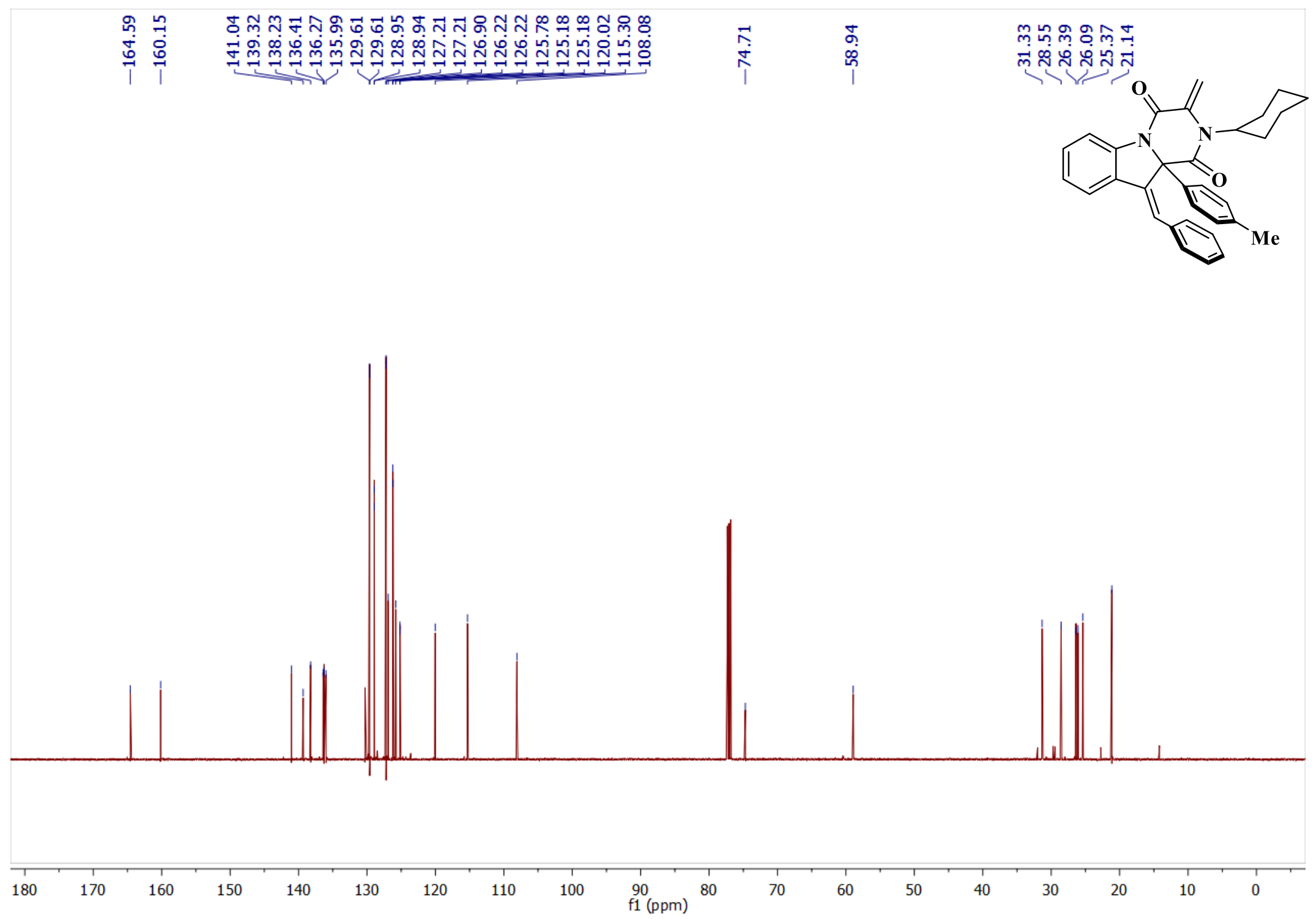

Figure S46. ${ }^{13} \mathrm{C}$ NMR(125 MHz, $\left.\mathrm{CDCl}_{3}\right) \mathbf{2 e}$ 


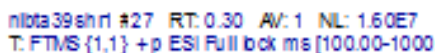

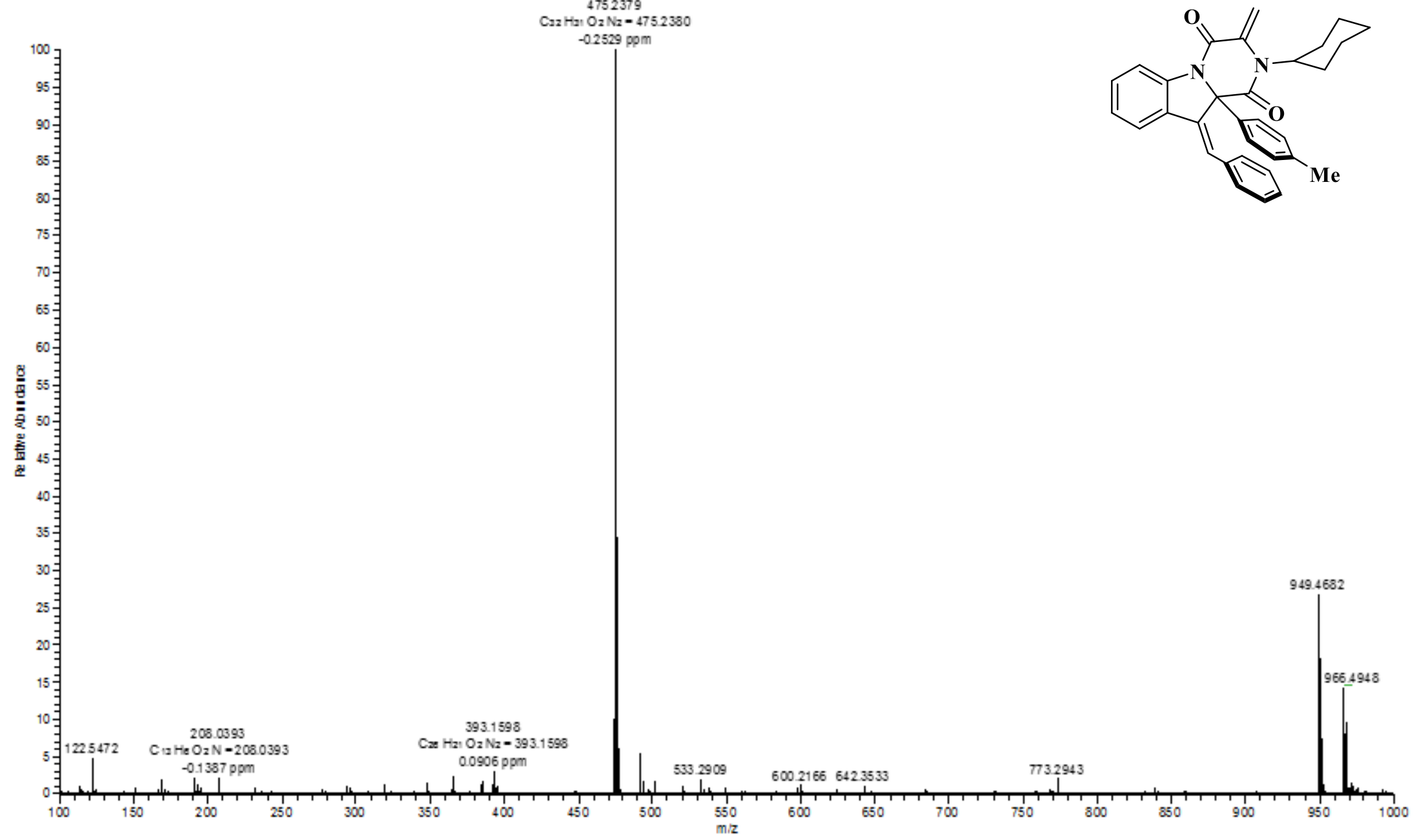

Figure S47. HRMS (ESI) 2e 


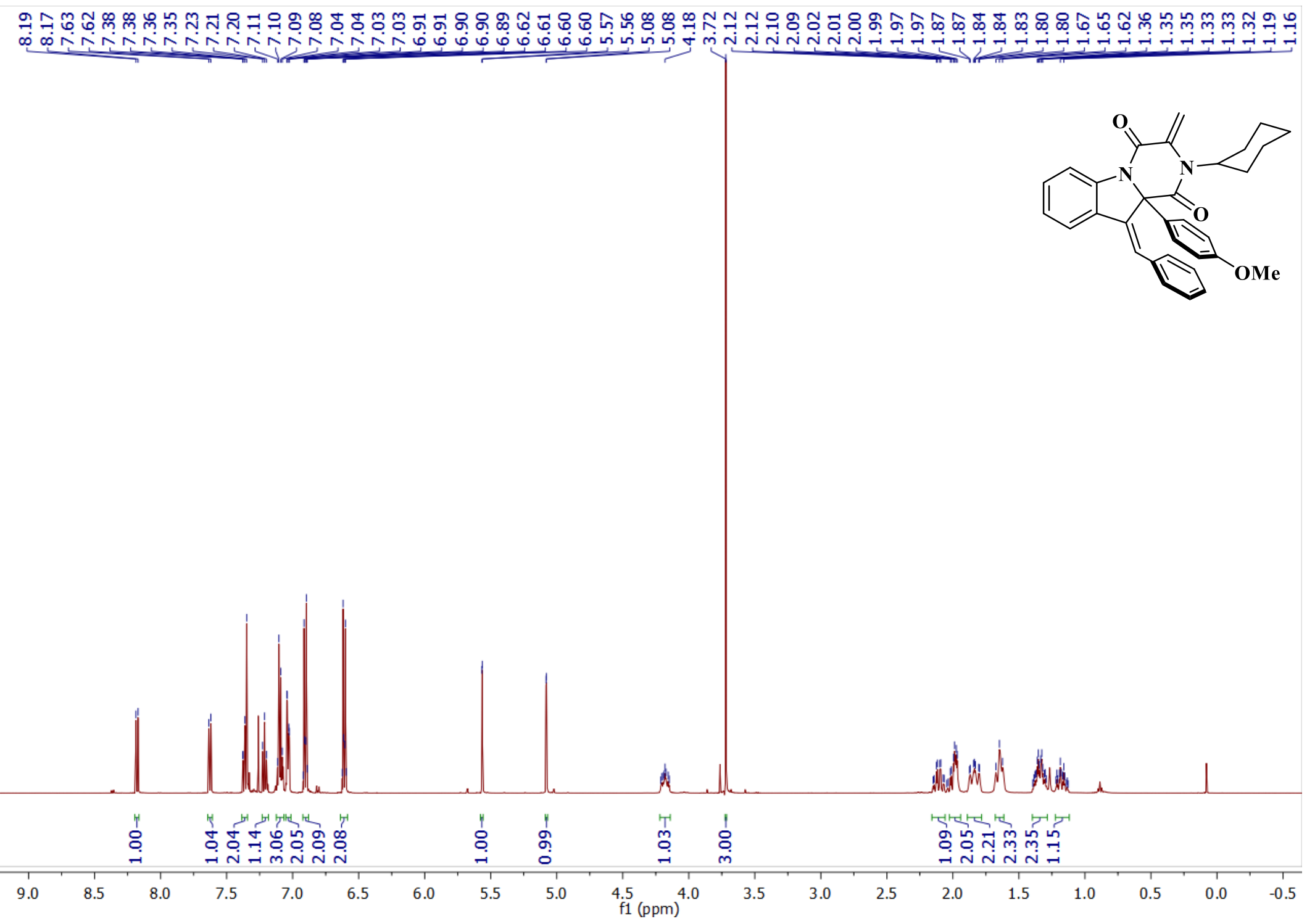

Figure $\mathrm{S} 48 .{ }^{1} \mathrm{H} \mathrm{NMR}\left(500 \mathrm{MHz}, \mathrm{CDCl}_{3}\right)$ 2f 


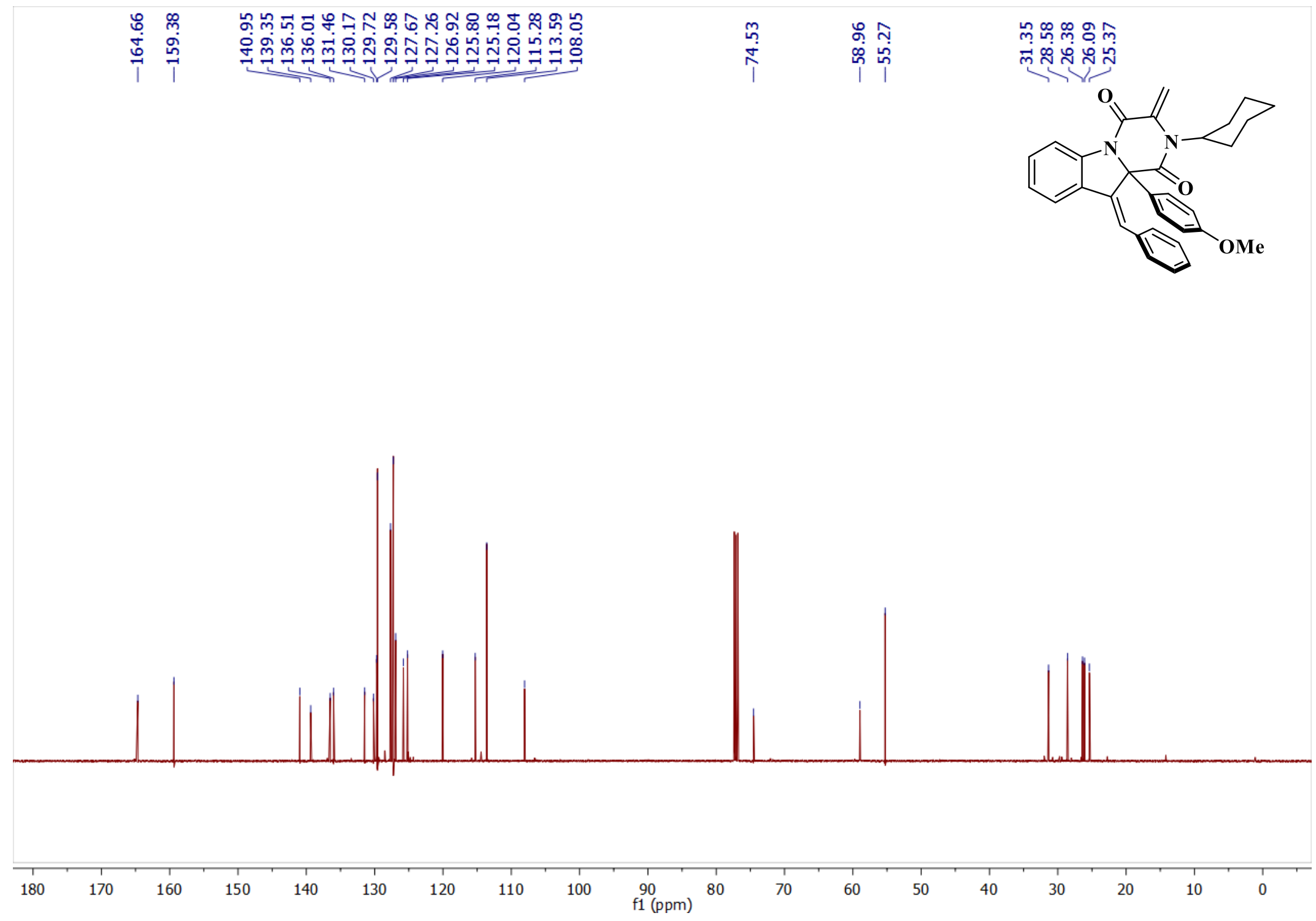

Figure $\mathrm{S} 49 .{ }^{13} \mathrm{C} \mathrm{NMR}\left(125 \mathrm{MHz}, \mathrm{CDCl}_{3}\right) \mathbf{2 f}$ 


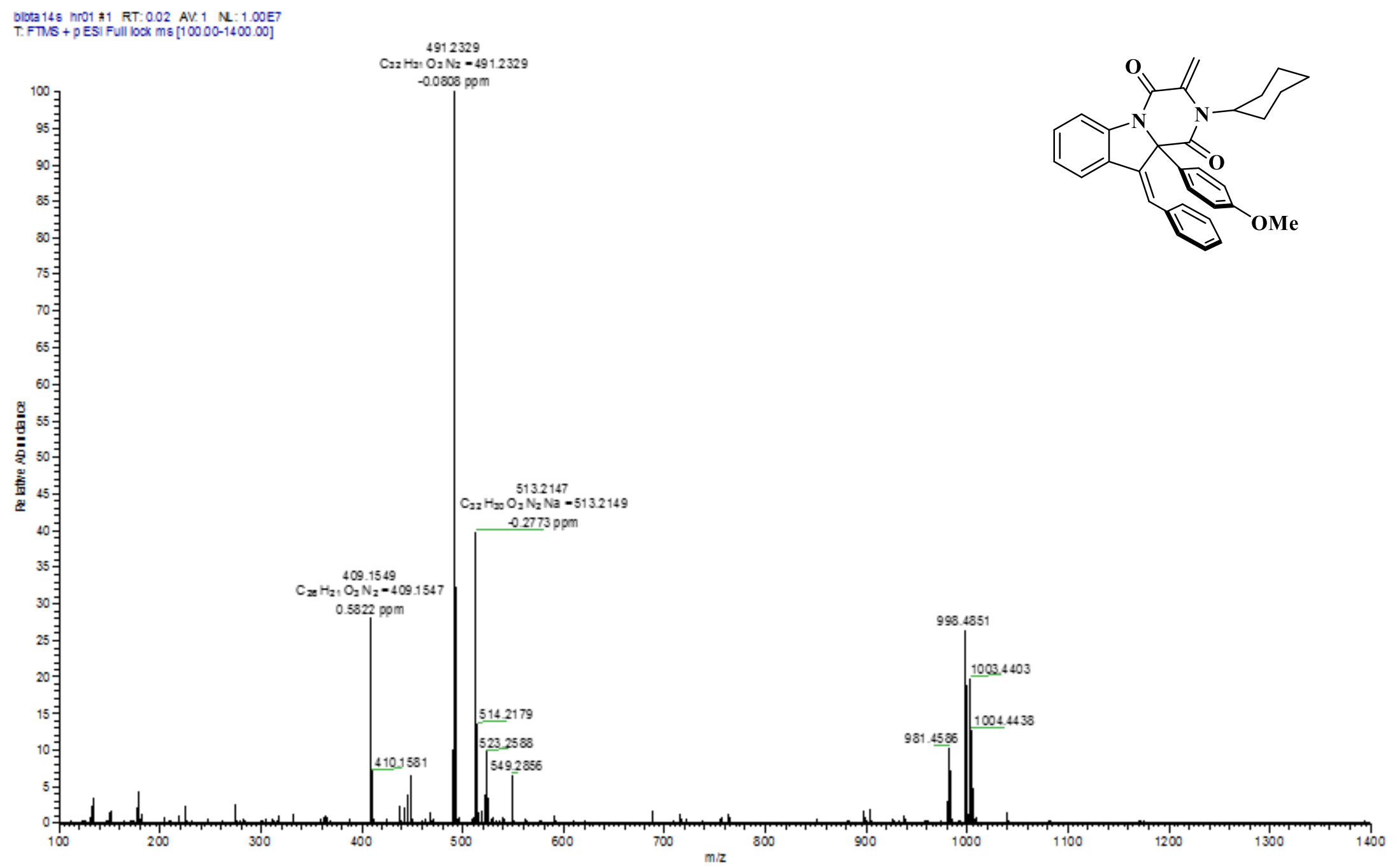

Figure S50. HRMS (ESI) 2f 

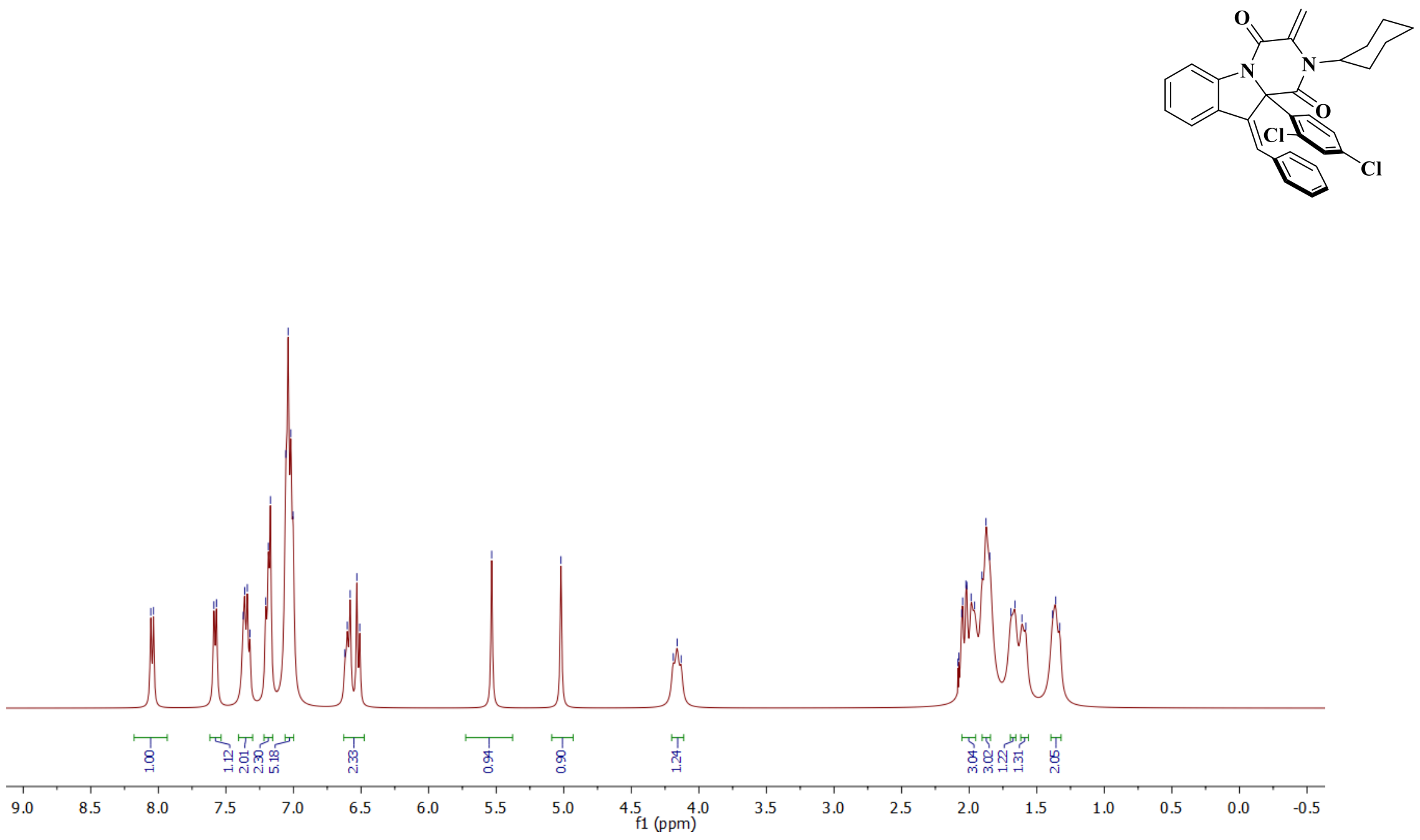

Figure $\mathrm{S} 51 .{ }^{1} \mathrm{H} \mathrm{NMR}\left(400 \mathrm{MHz}, \mathrm{CDCl}_{3}\right) \mathbf{2 g}$ 

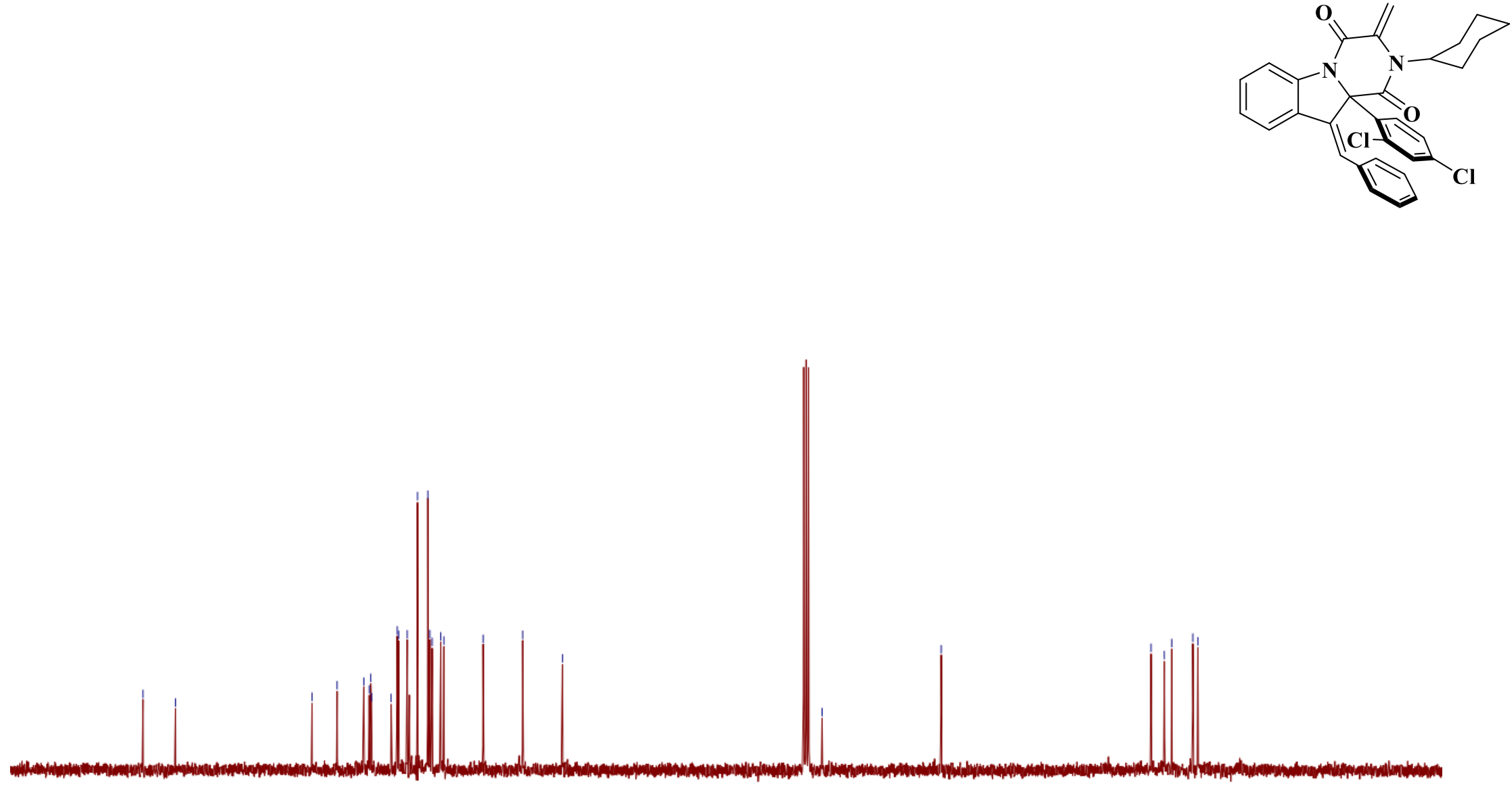

1

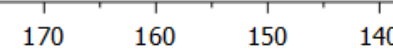

130

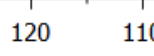

100

$\stackrel{90}{\mathrm{f} 1(\mathrm{ppm})}$

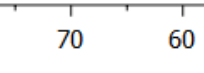

50

40

30

20

10

Figure $\mathrm{S} 52 .{ }^{13} \mathrm{C} \mathrm{NMR}\left(100 \mathrm{MHz}, \mathrm{CDCl}_{3}\right) \mathbf{2 g}$ 
nlota 40 shn 2 2 35 RT: 0.39 AV: 1 NL: $1.25 E 7$
T. FT/S $\{2,1\}+p$ ESI Full bok ms $[10000-1000.00]$

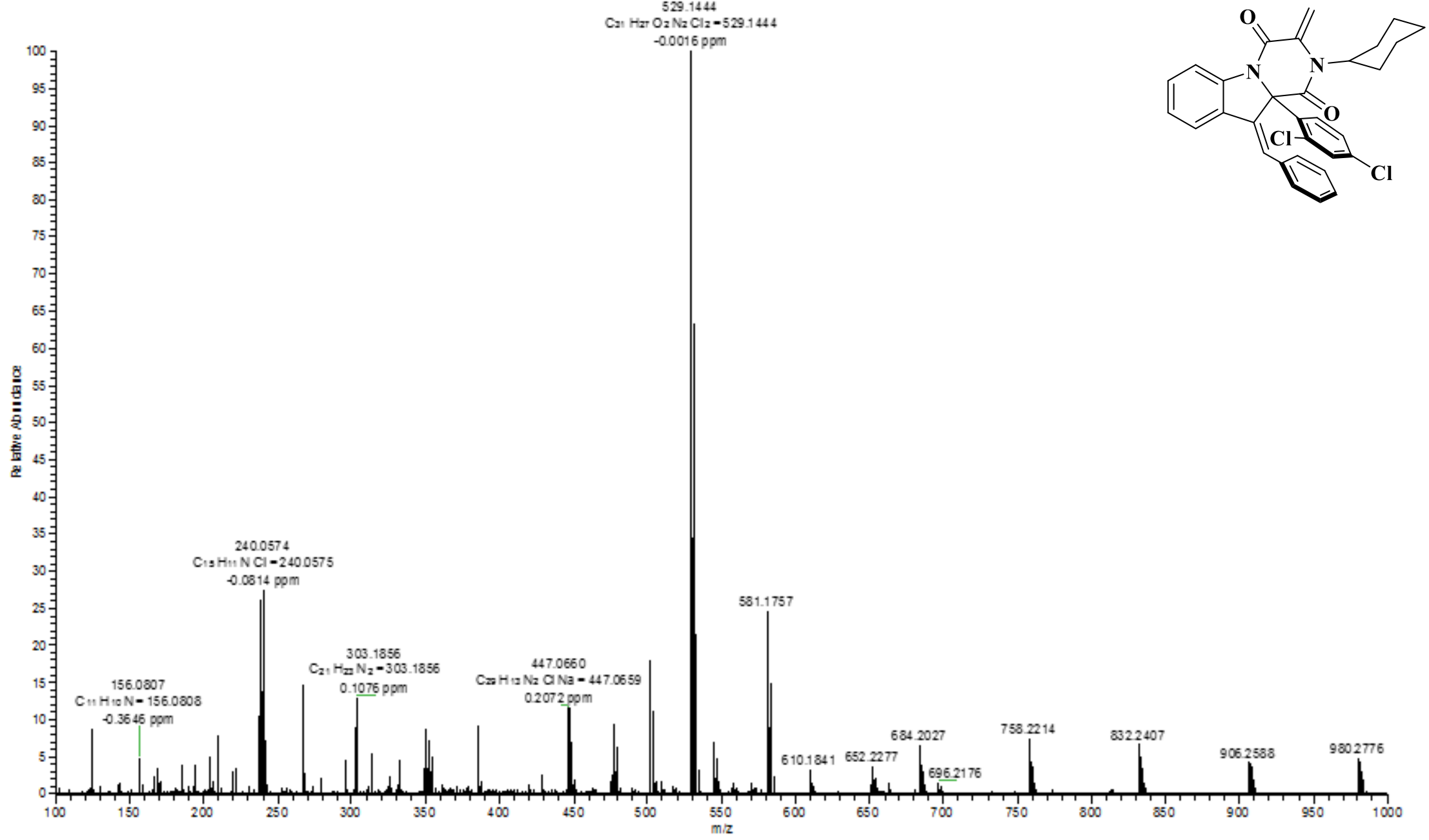

Figure S53. HRMS (ESI) 2g 


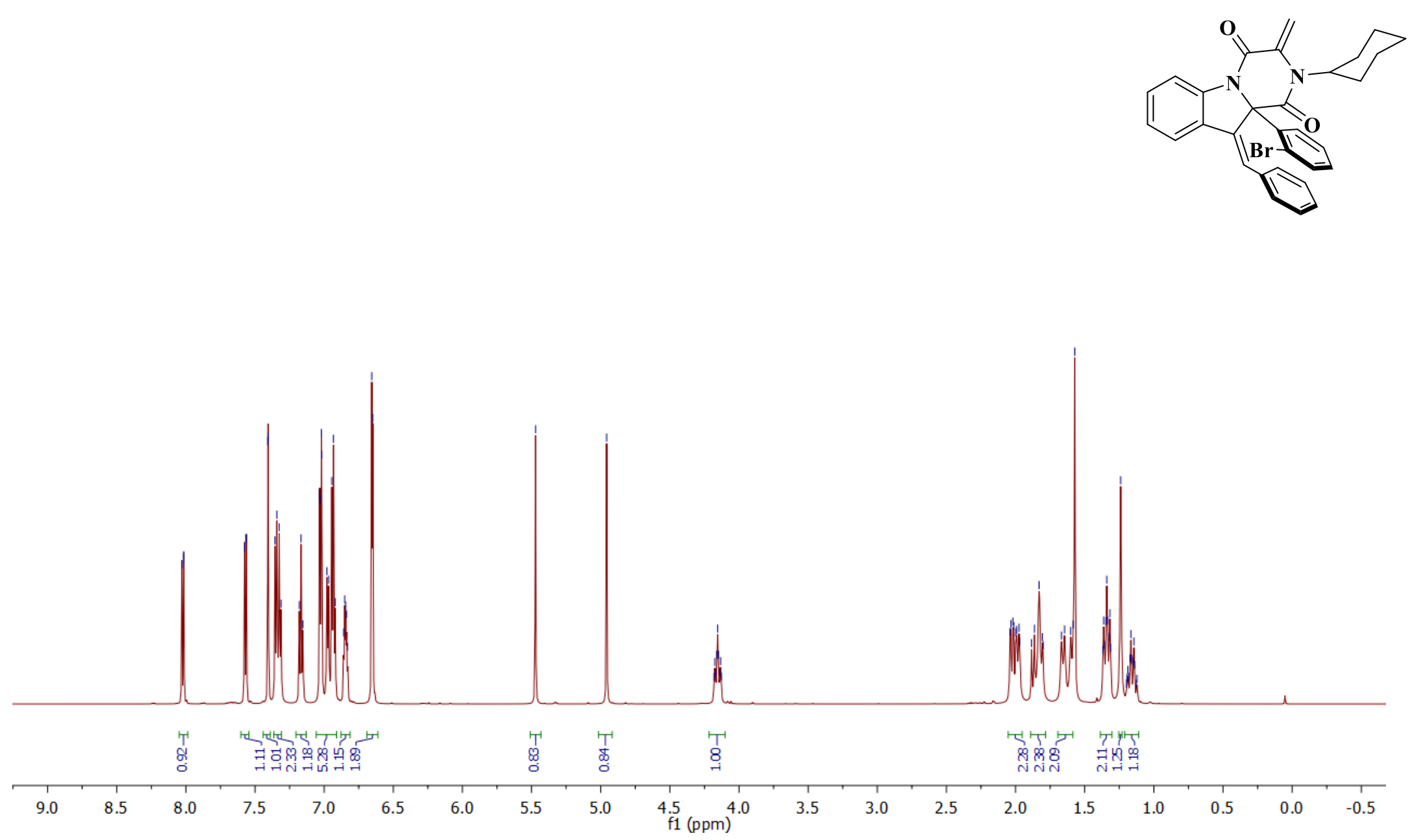

Figure S54. ${ }^{1} \mathrm{H} \mathrm{NMR}\left(600 \mathrm{MHz}, \mathrm{CDCl}_{3}\right) \mathbf{2 h}$ 

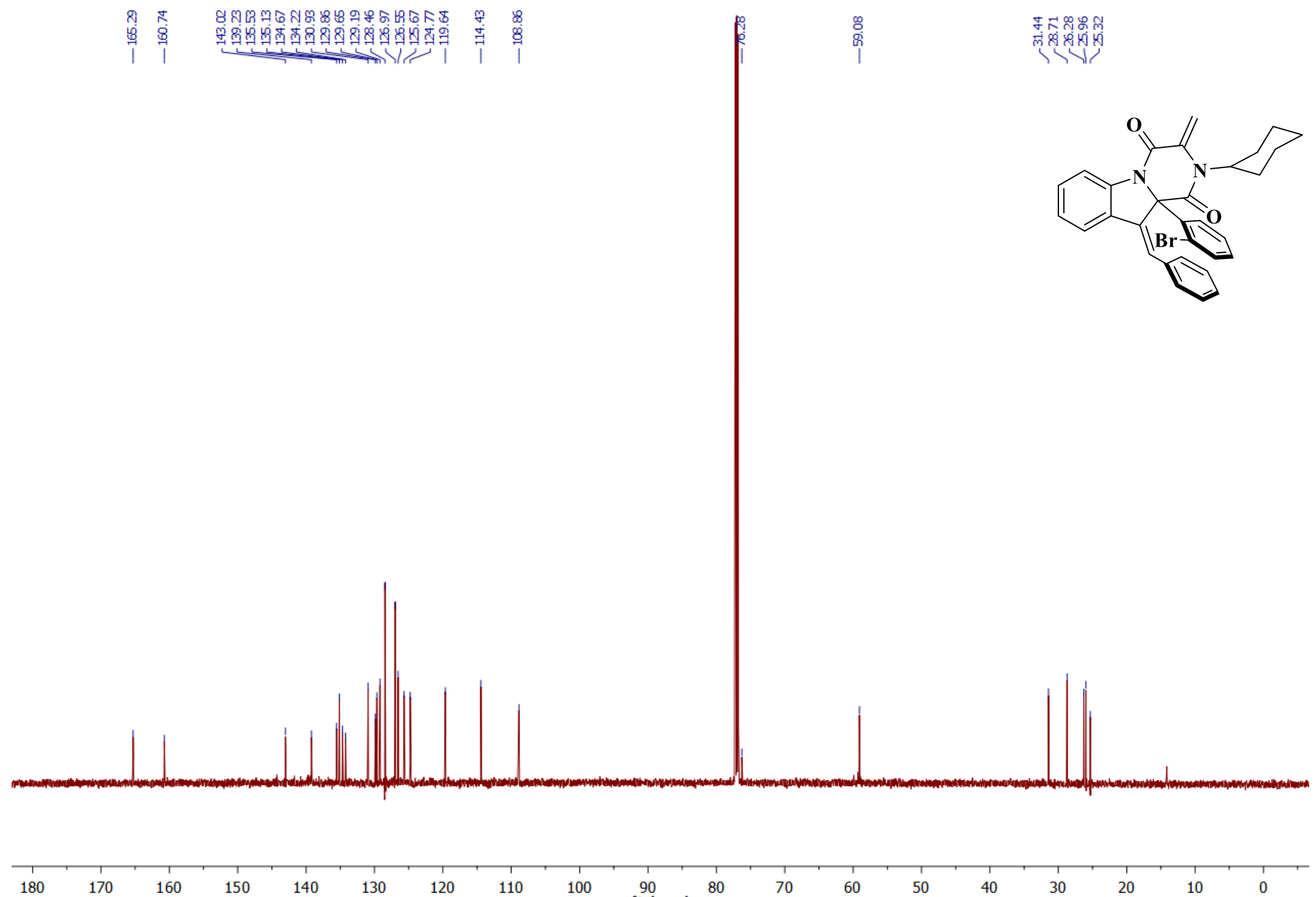

120

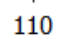

100

90
$f 1(p p m)$

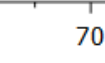

60

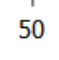

40

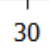

20

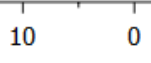

Figure $555 .{ }^{13} \mathrm{C} \mathrm{NMR}\left(150 \mathrm{MHz}, \mathrm{CDCl}_{3}\right) \mathbf{2 h}$ 


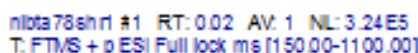

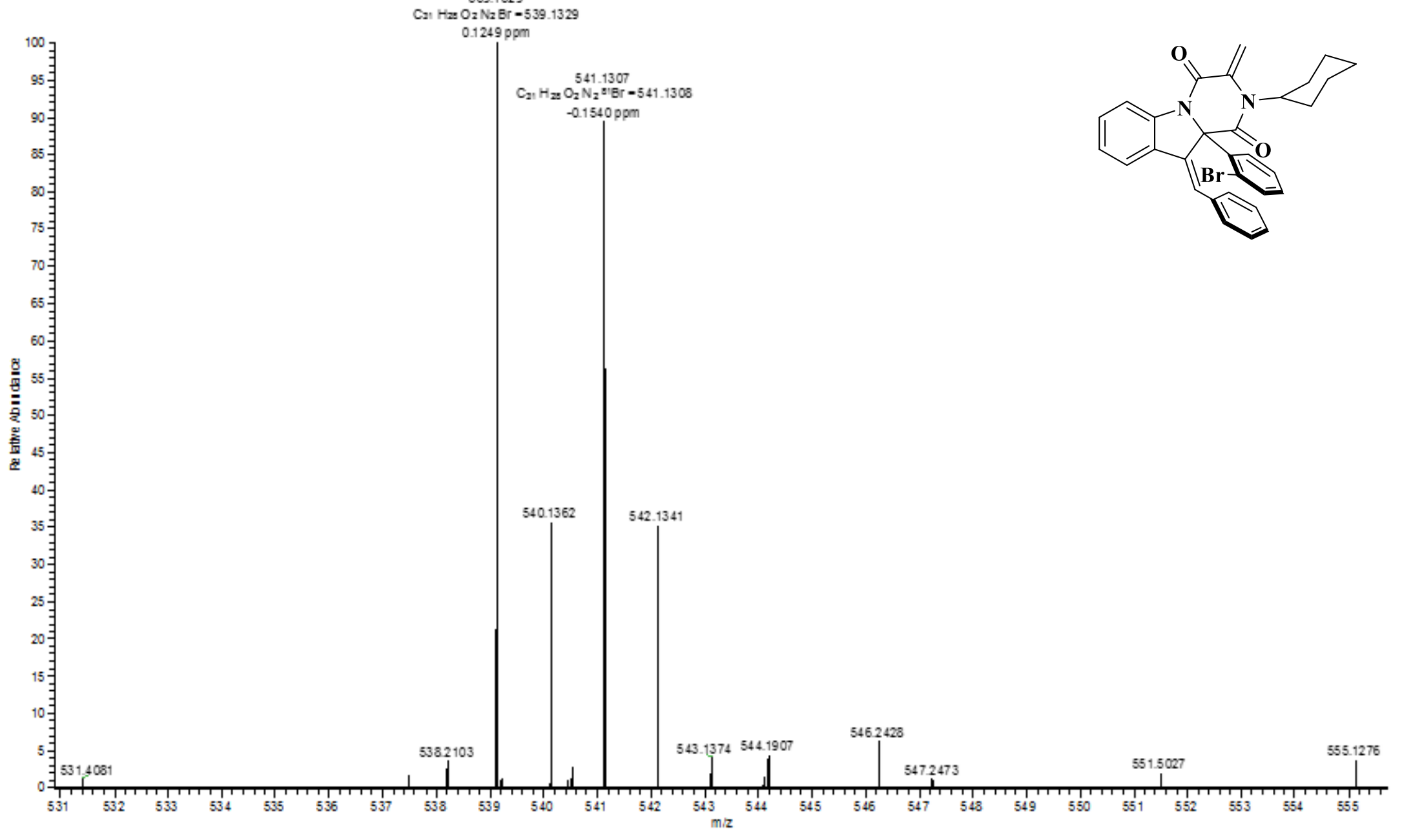

Figure S56. HRMS (ESI) 2h 

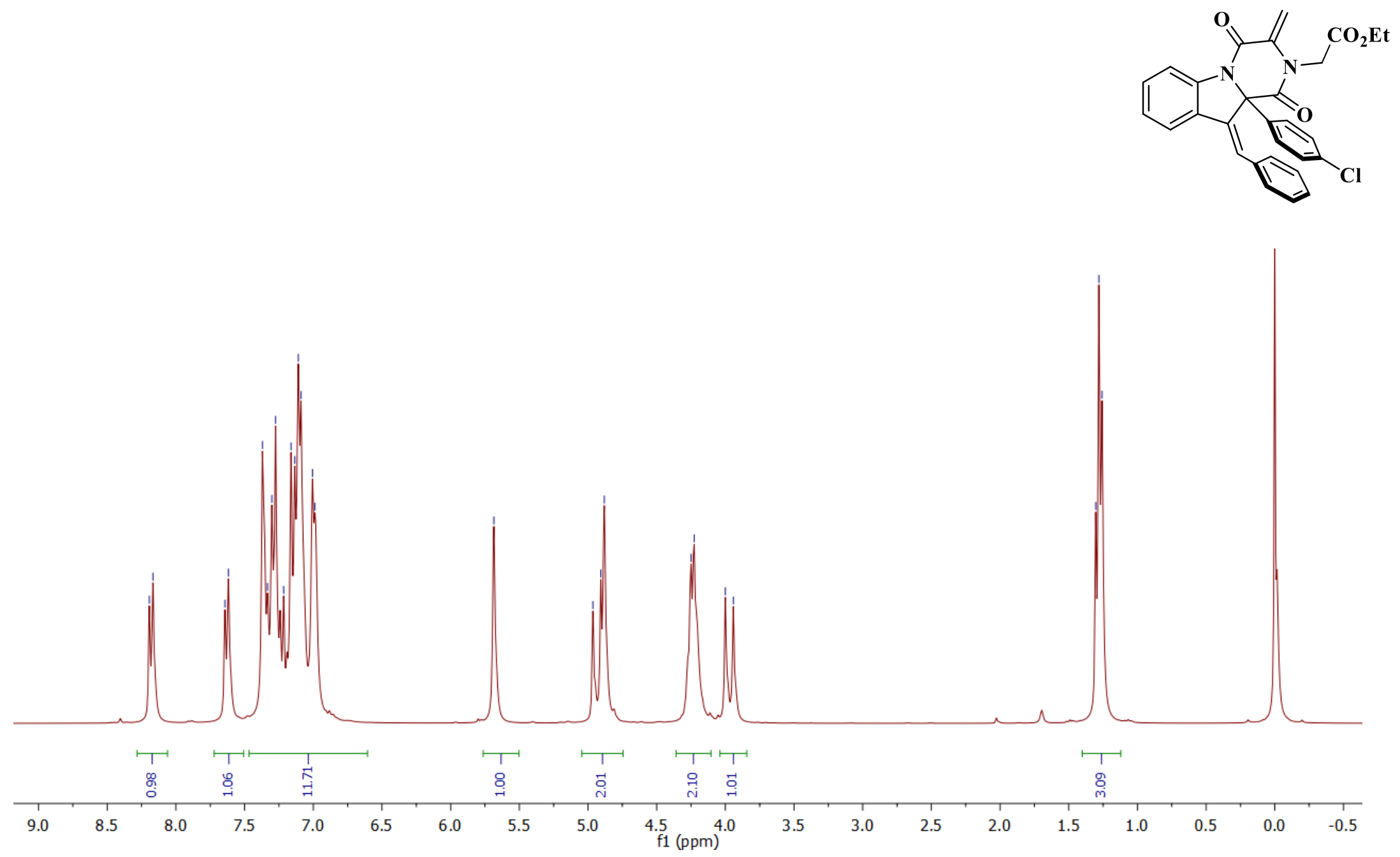

Figure S57. ${ }^{1} \mathrm{H} \mathrm{NMR}\left(300 \mathrm{MHz}, \mathrm{CDCl}_{3}\right) \quad 2 \mathbf{i}$ 


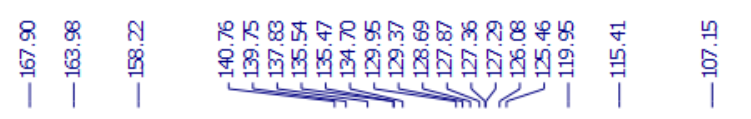

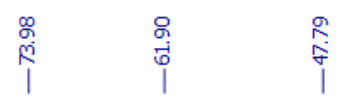
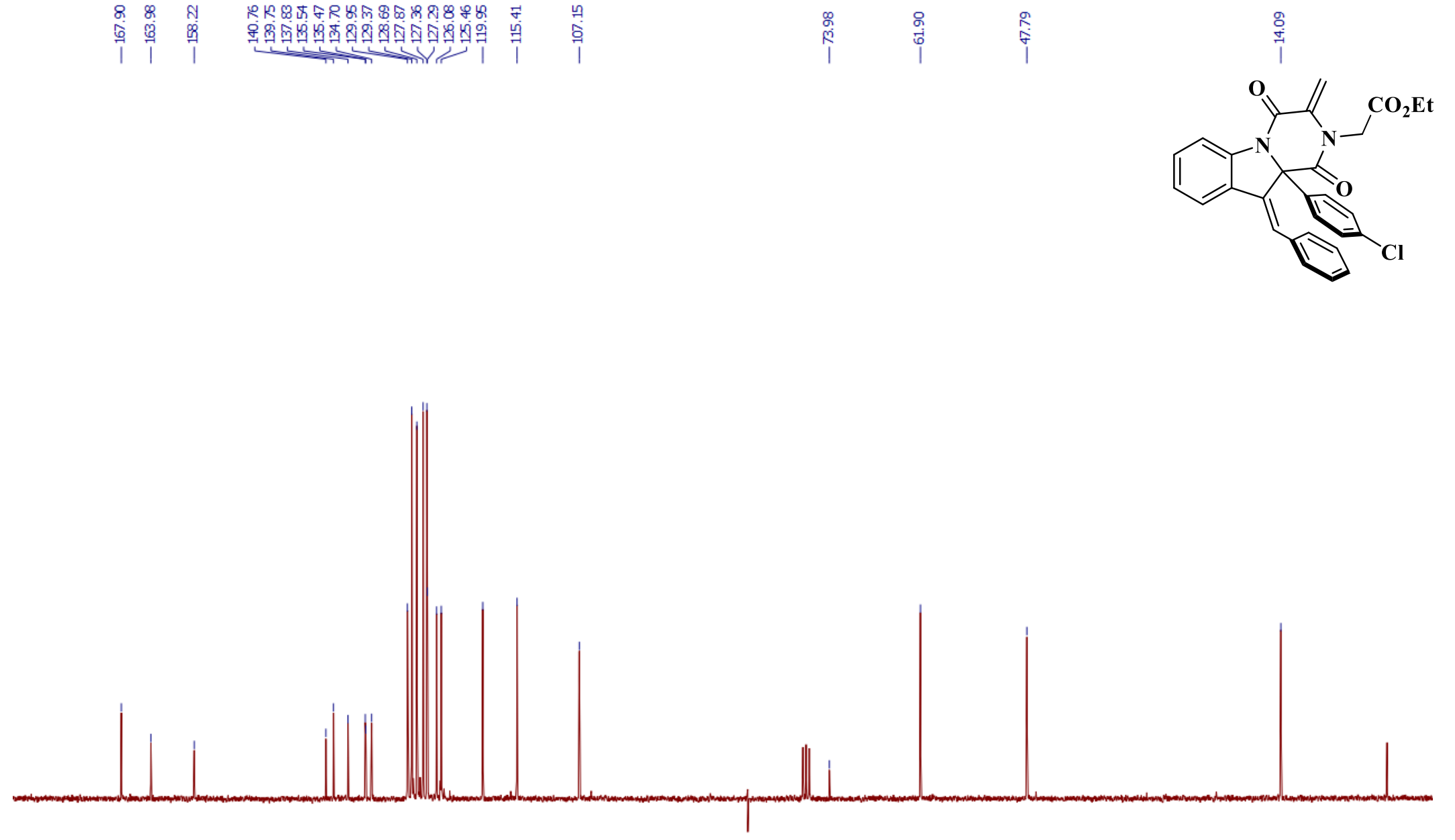

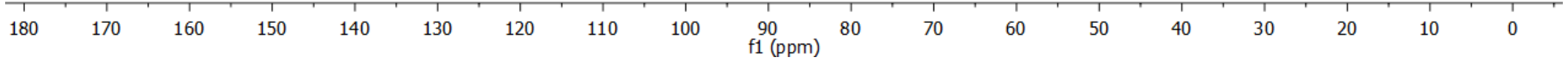

Figure S58. ${ }^{13} \mathrm{C} \mathrm{NMR}\left(75 \mathrm{MHz}, \mathrm{CDCl}_{3}\right) \mathbf{2 i}$ 


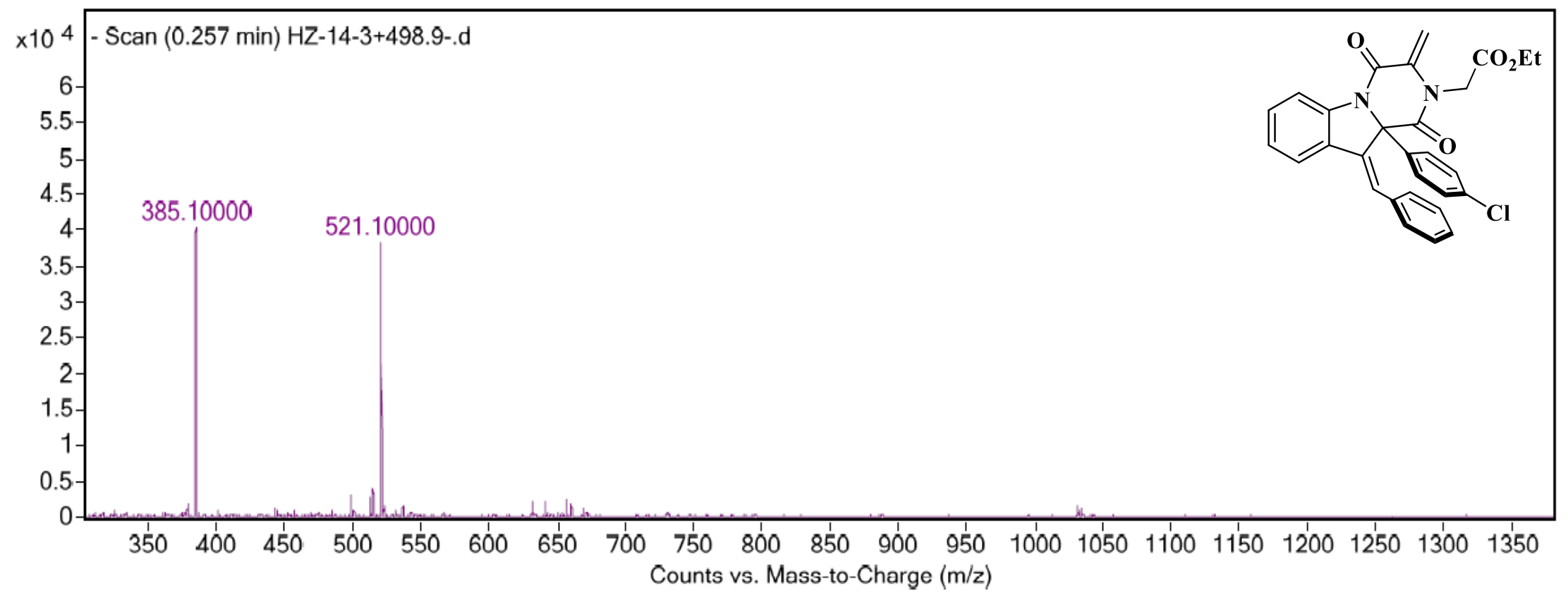

Figure S59. MS (ESI) 2i 


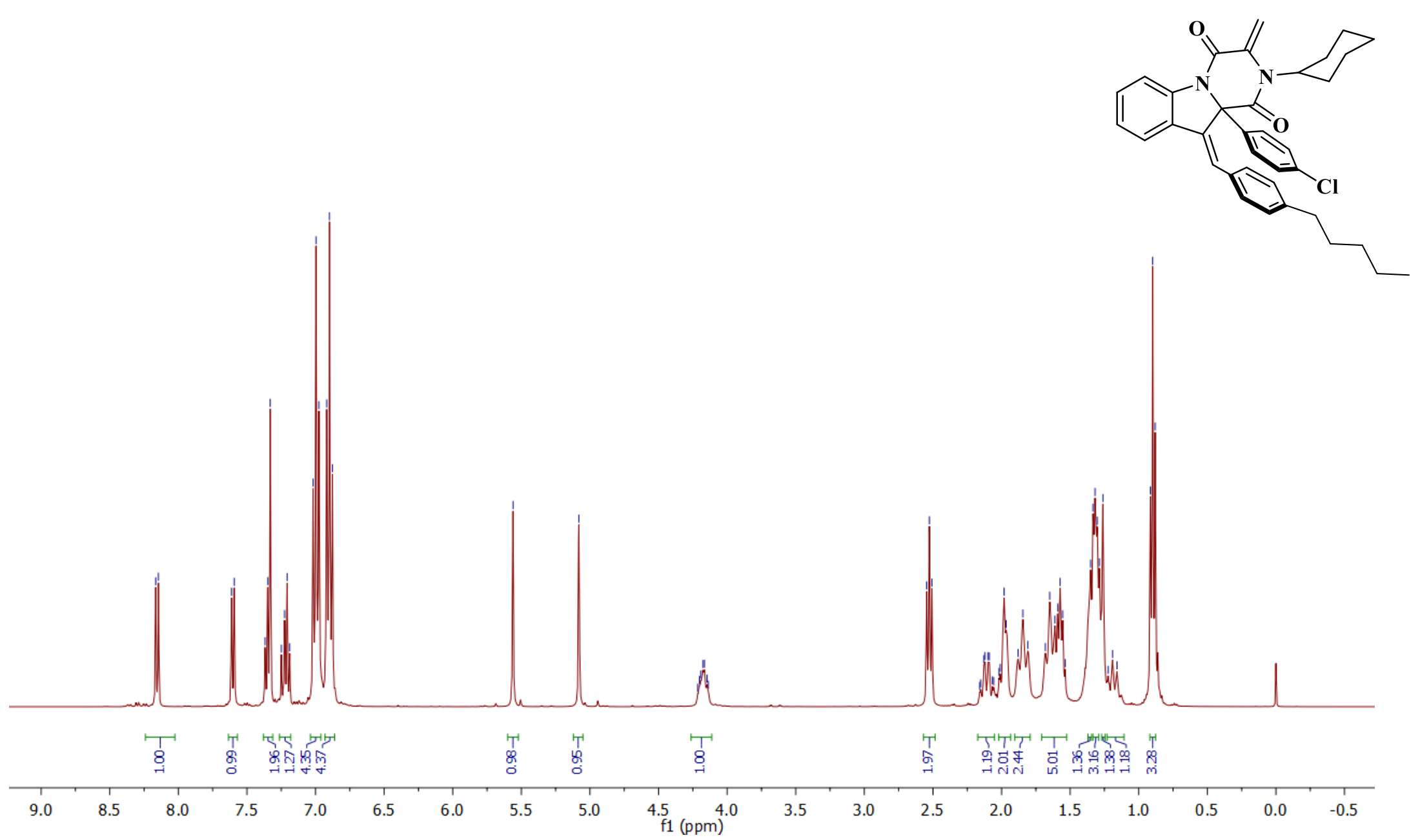

Figure $\mathrm{S} 60 .{ }^{1} \mathrm{H} \mathrm{NMR}\left(400 \mathrm{MHz}, \mathrm{CDCl}_{3}\right) \mathbf{2} \mathbf{j}$ 

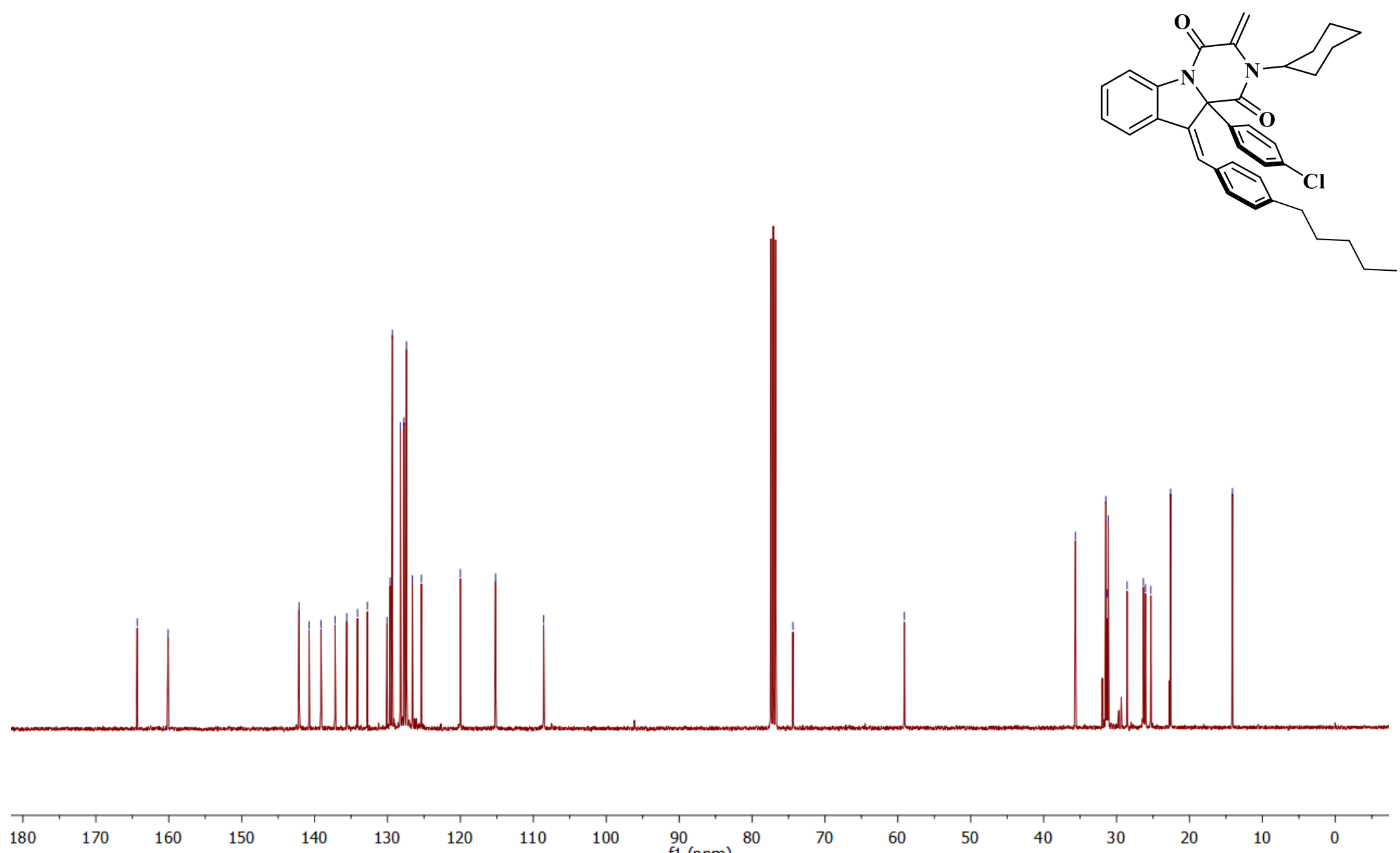

100

$\mathrm{f1}^{90}{ }^{80}$
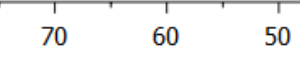

40

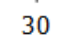

20

Figure $\mathrm{S} 61 .{ }^{13} \mathrm{C} \mathrm{NMR}\left(100 \mathrm{MHz}, \mathrm{CDCl}_{3}\right) \mathbf{2} \mathbf{j}$ 


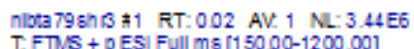

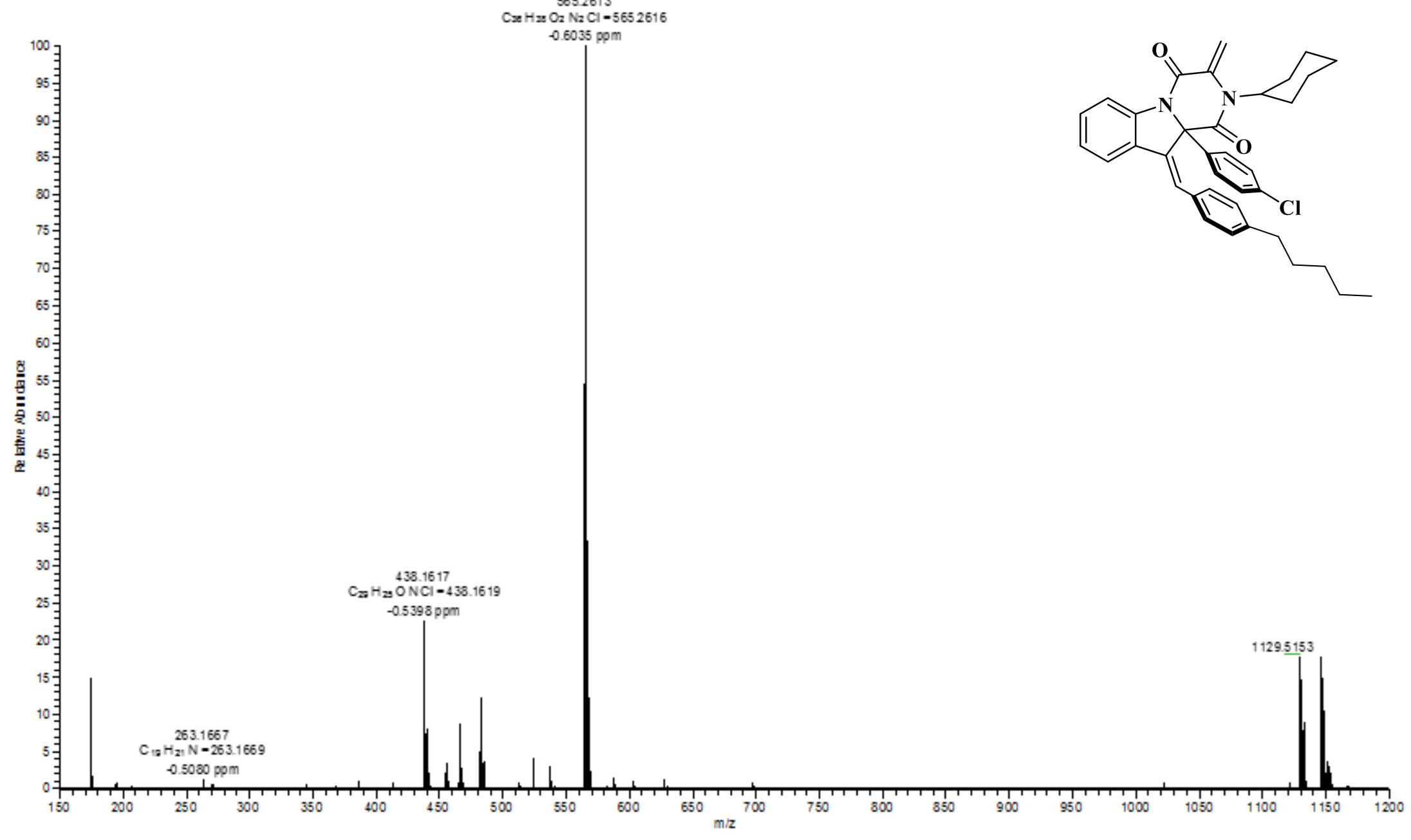

Figure S62. HRMS (ESI) 2j 

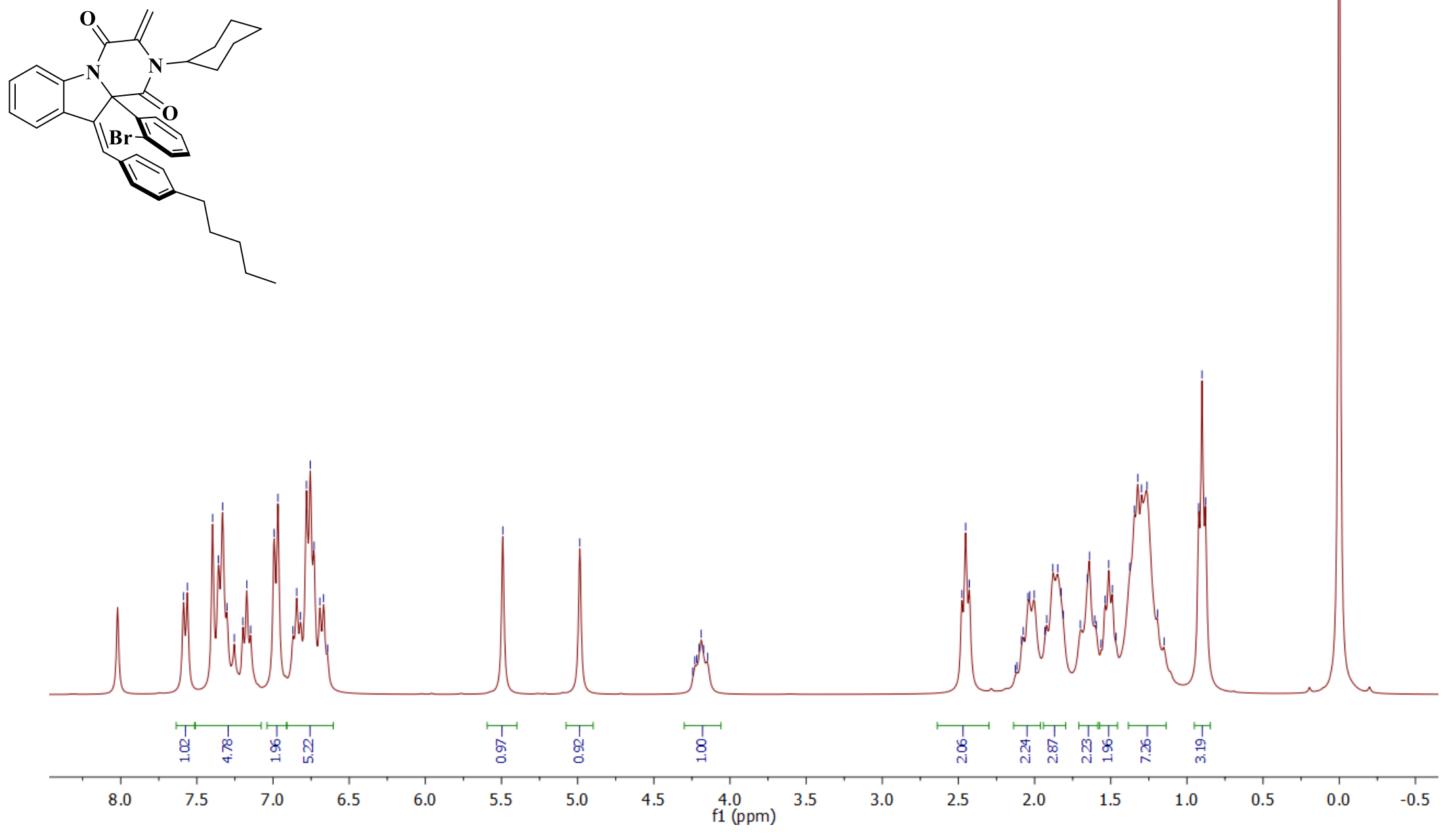

Figure S63. ${ }^{1} \mathrm{H} \mathrm{NMR}\left(300 \mathrm{MHz}, \mathrm{CDCl}_{3}\right) \mathbf{2 k}$ 


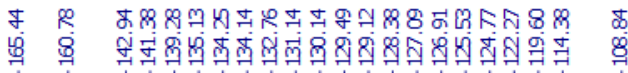

। I v

180

$\begin{array}{llllll}170 & 160 & 150 & 140 & 130 & 120\end{array}$

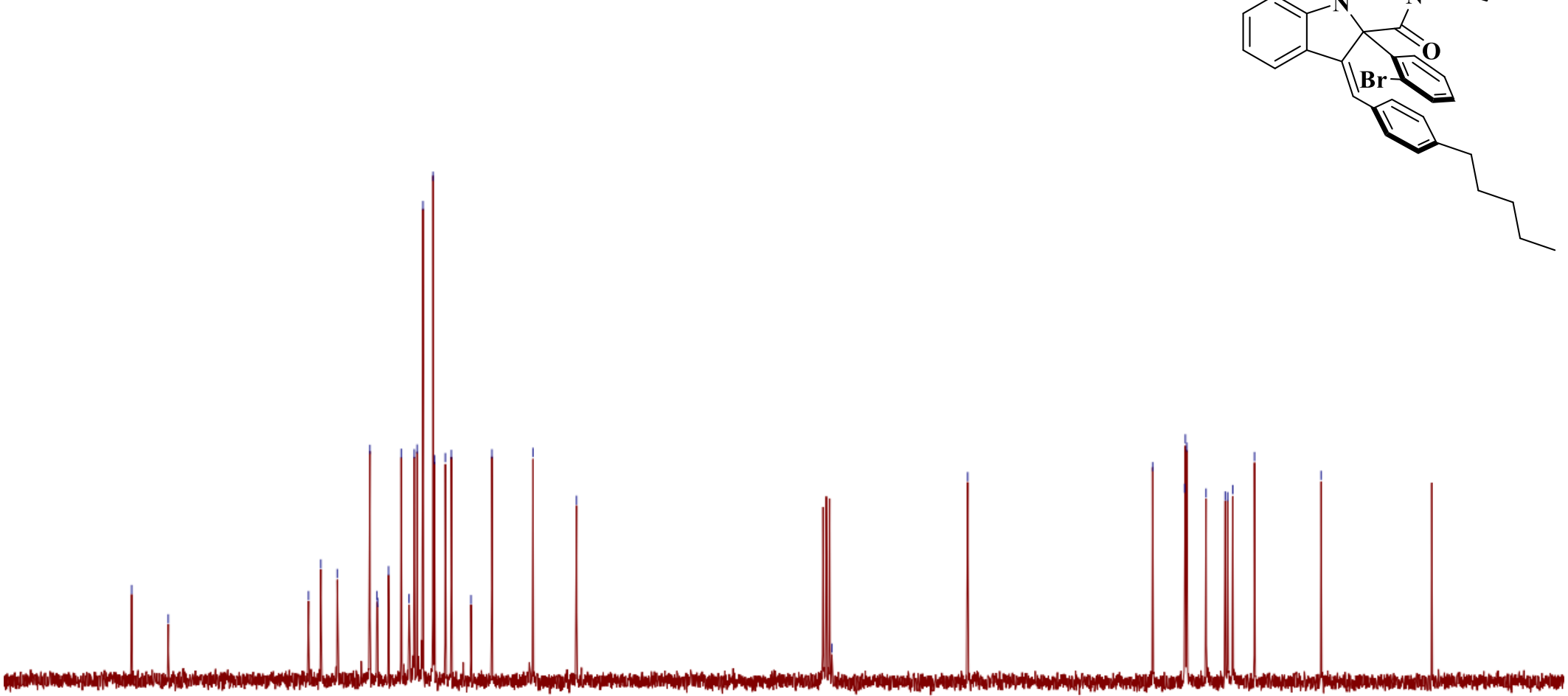

i

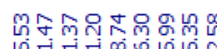

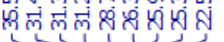
$\rightarrow 11$

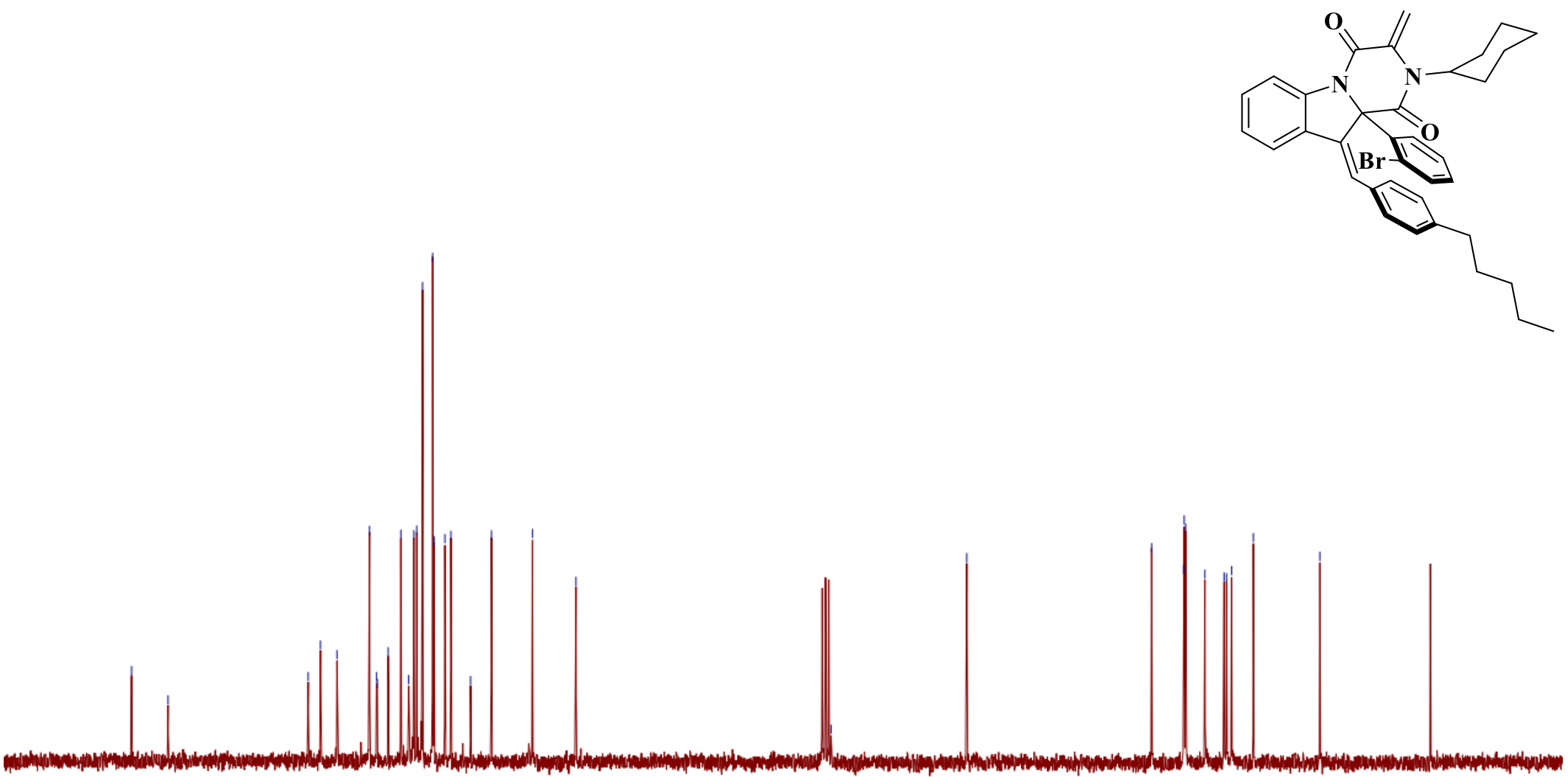

90

$f 1\left(\begin{array}{c}80 \\ \text { ppm) }\end{array}\right.$

$60 \quad 50$

40

30

20

10

0

Figure $\mathrm{S} 64 .{ }^{13} \mathrm{C} \mathrm{NMR}\left(75 \mathrm{MHz}, \mathrm{CDCl}_{3}\right) \mathbf{2 k}$ 
nitota $84 \sin 1=1$ RT: 0.02 AN. 1 NL: 87966

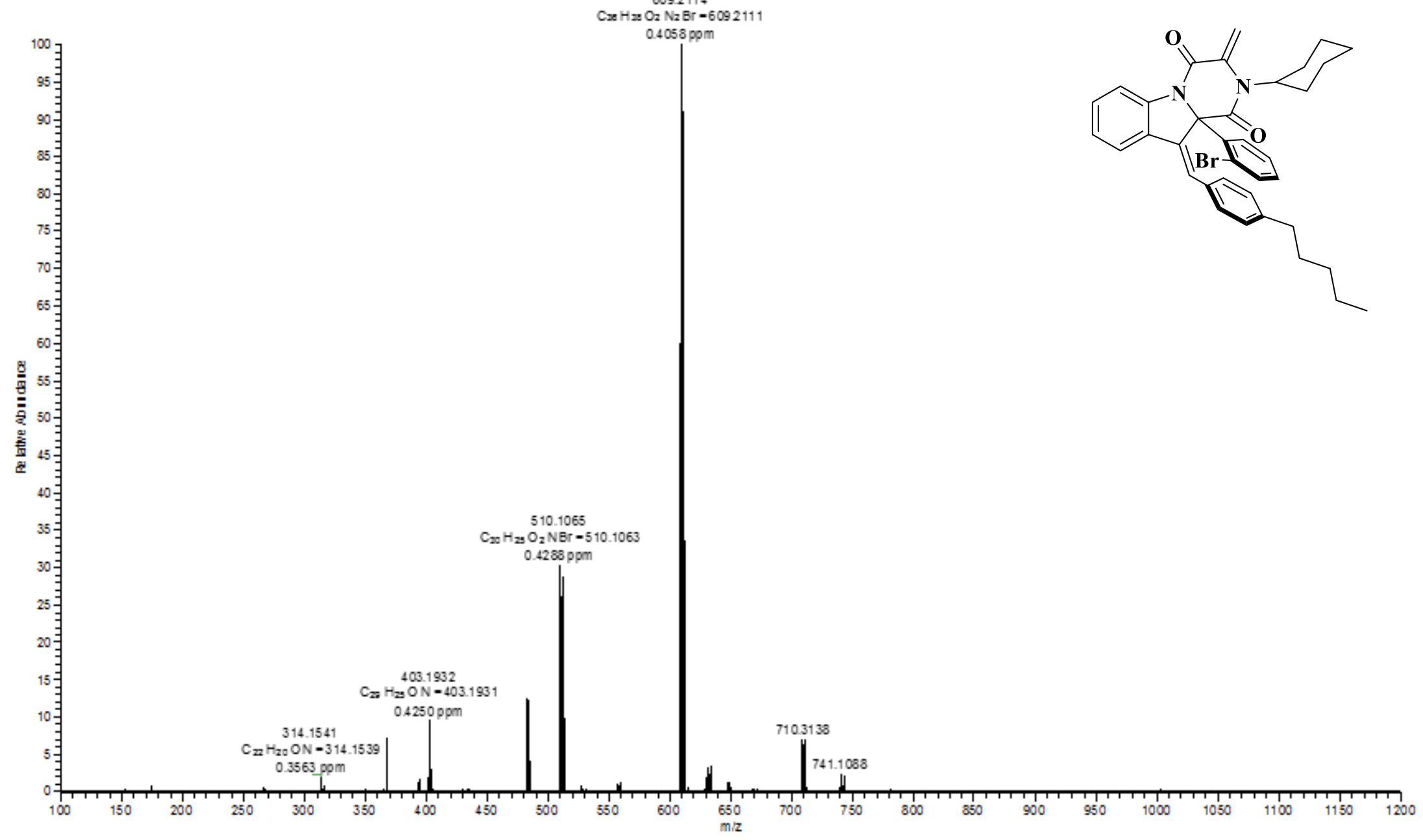

Figure S65. HRMS (ESI) 2k 

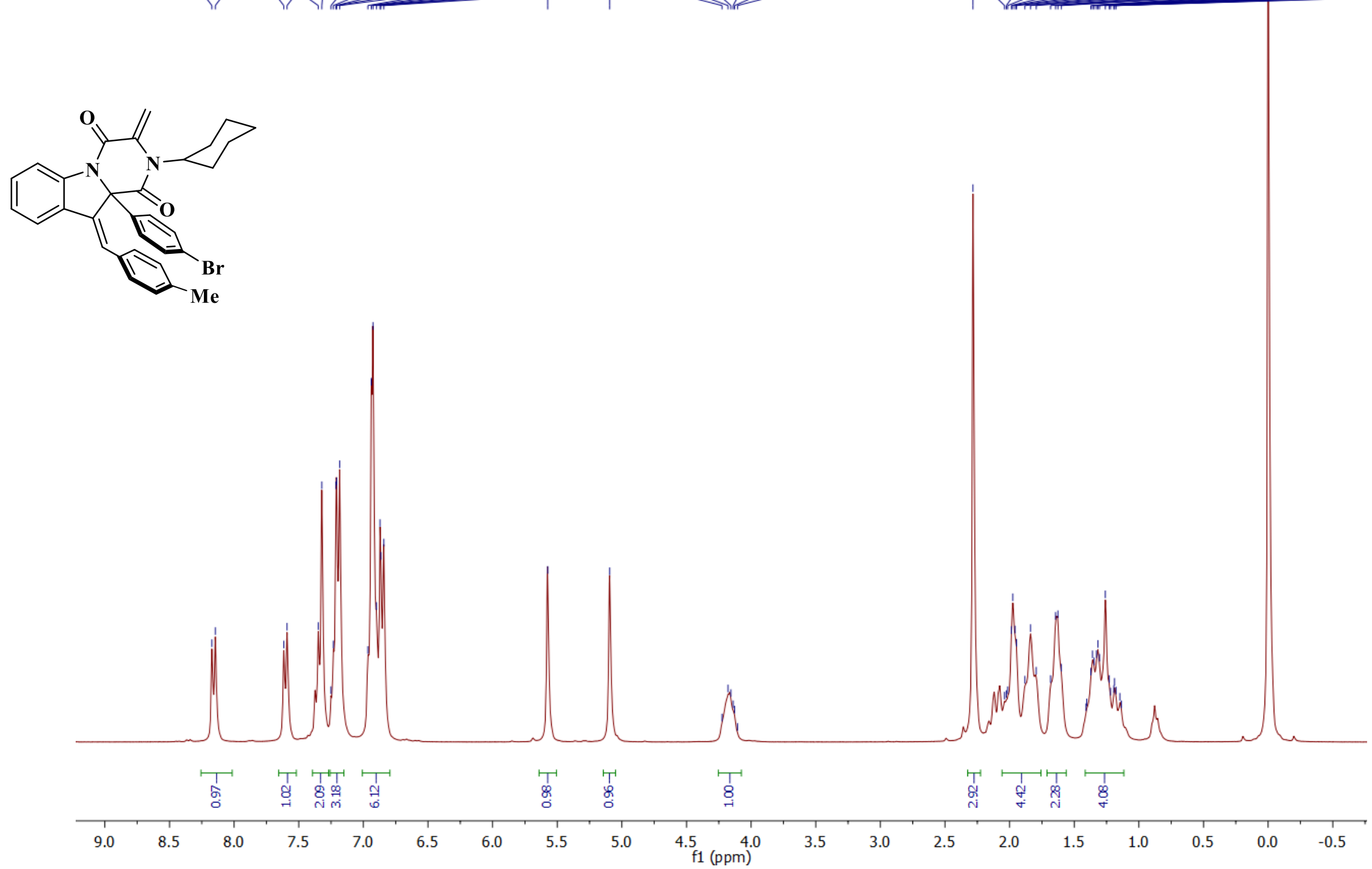

Figure S66. ${ }^{1} \mathrm{H} \mathrm{NMR}\left(300 \mathrm{MHz}, \mathrm{CDCl}_{3}\right) 2 \mathbf{I}$ 

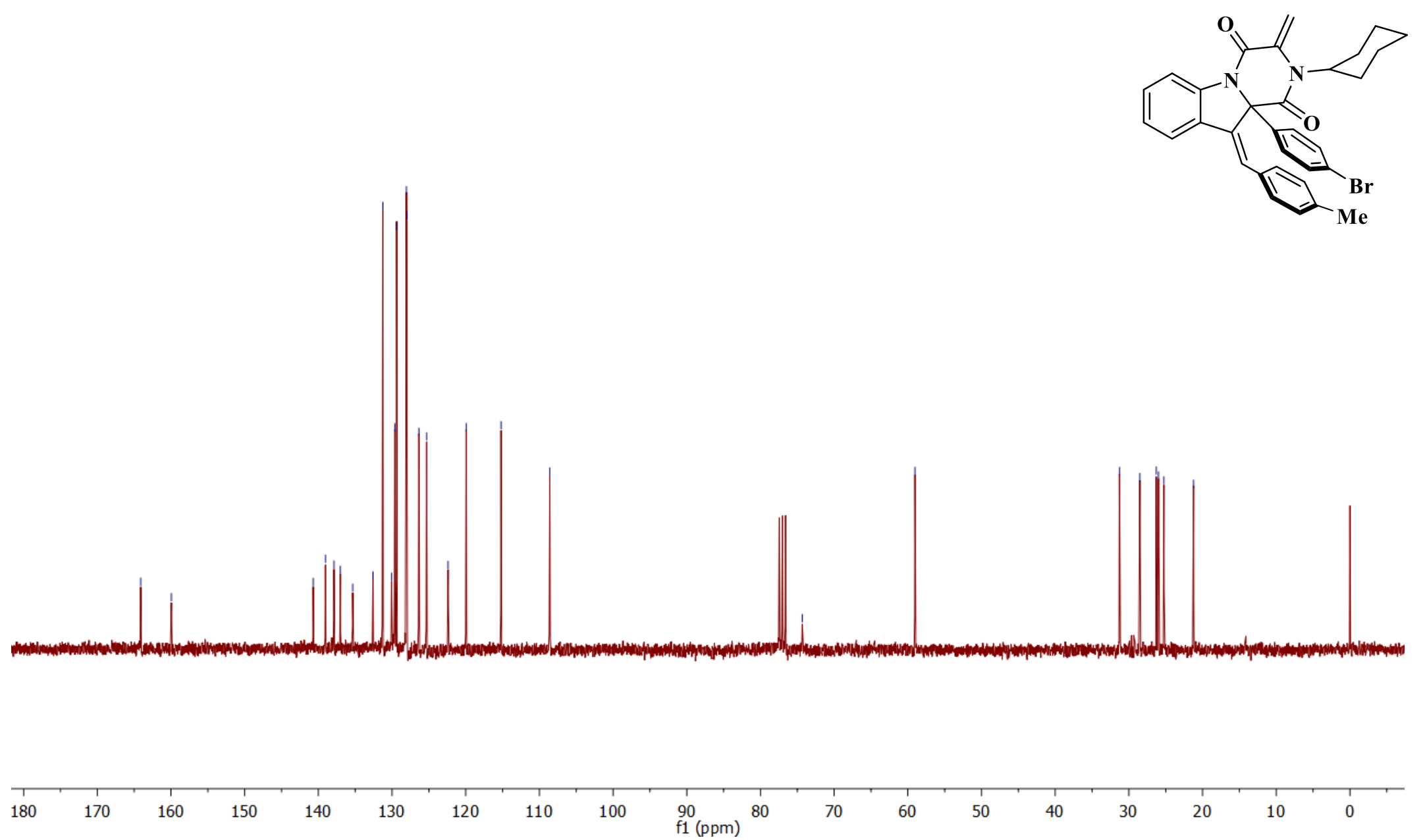

Figure S67. ${ }^{13} \mathrm{C} \mathrm{NMR}\left(75 \mathrm{MHz}, \mathrm{CDCl}_{3}\right) 2 \mathbf{I}$ 


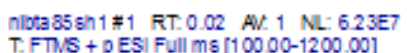

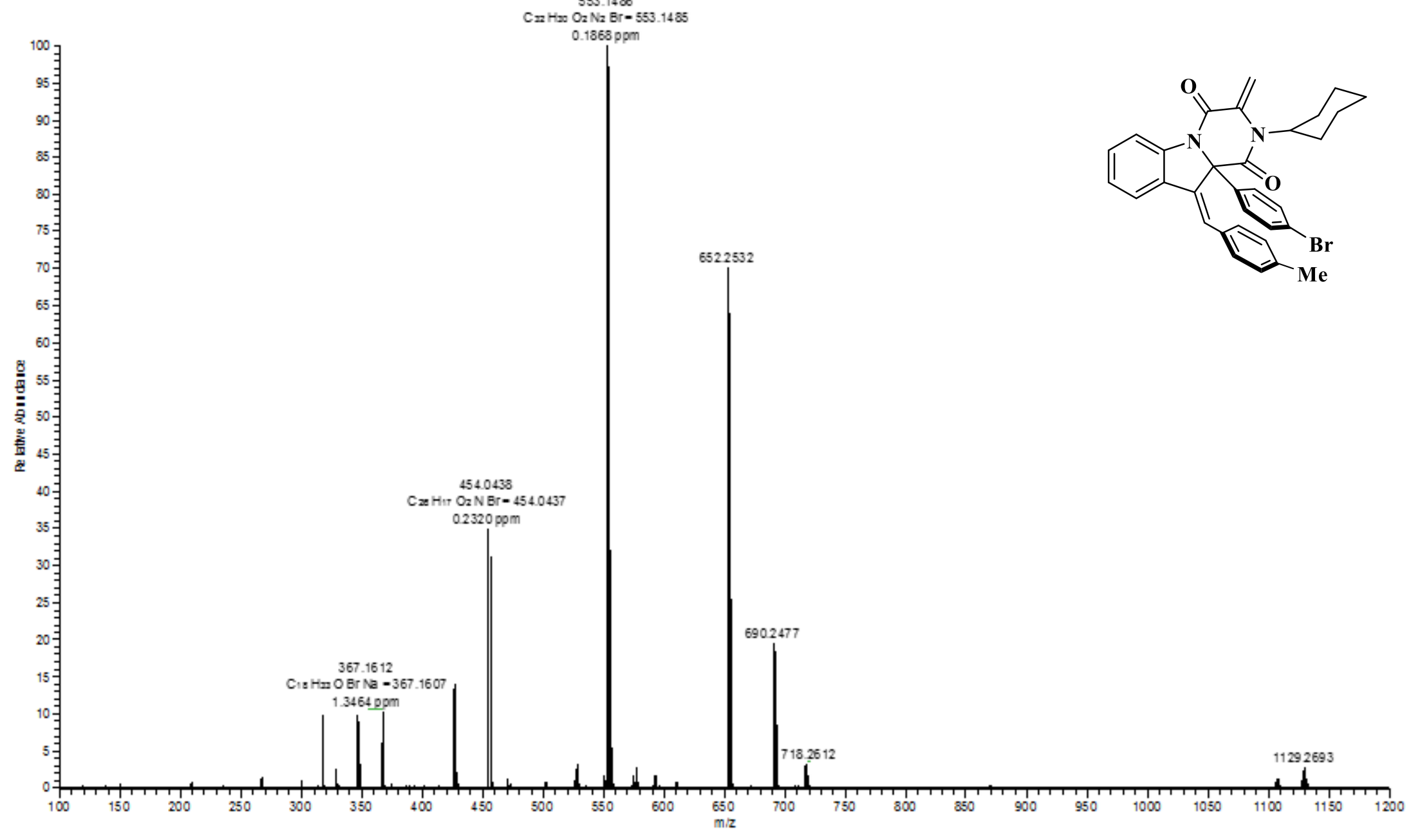

Figure S68. HRMS(ESI) 21 


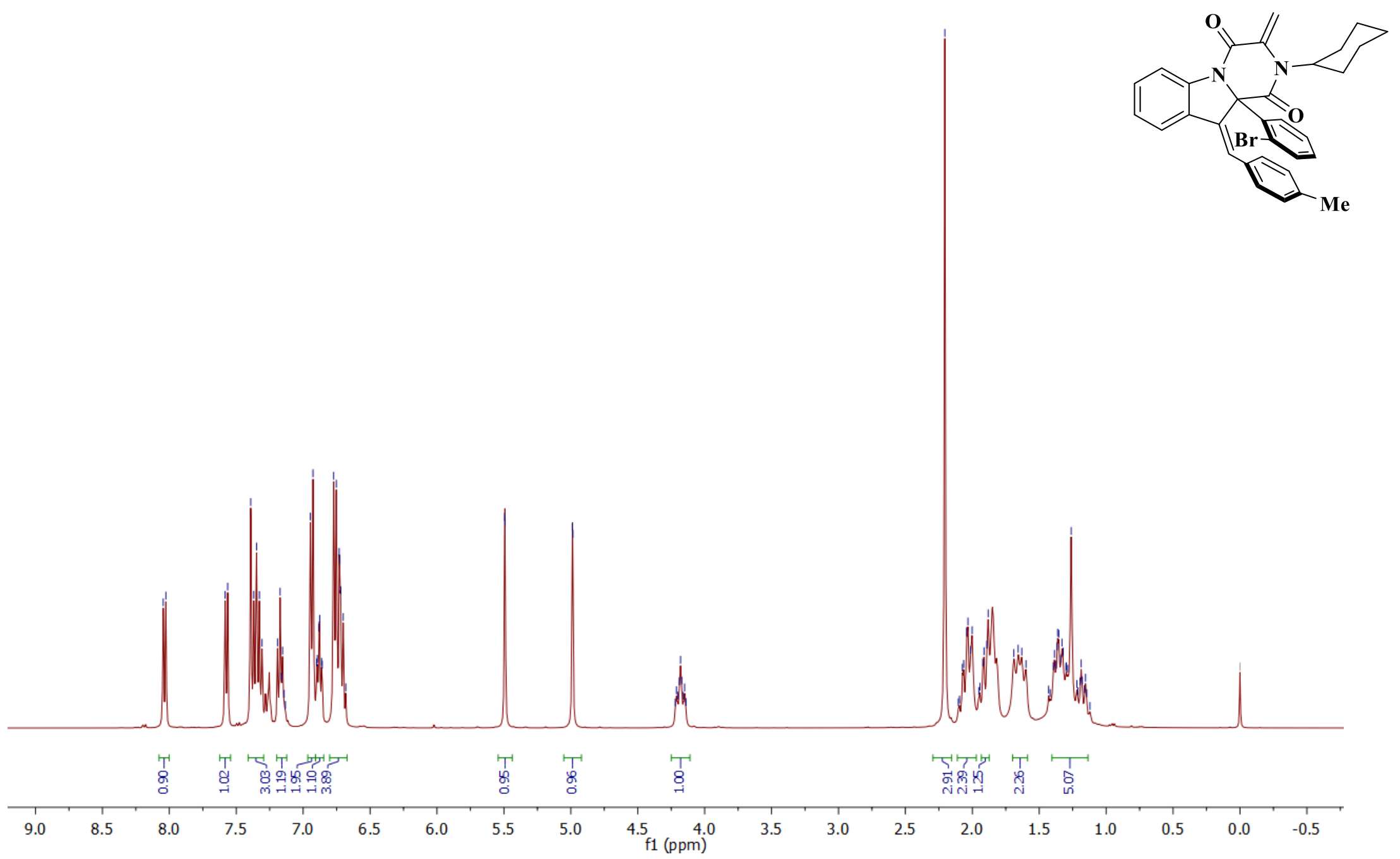

Figure S69. ${ }^{1} \mathrm{H} \mathrm{NMR}\left(400 \mathrm{MHz}, \mathrm{CDCl}_{3}\right) \mathbf{2 m}$ 


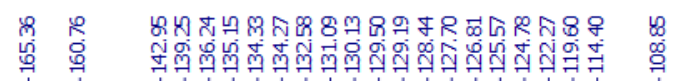

।
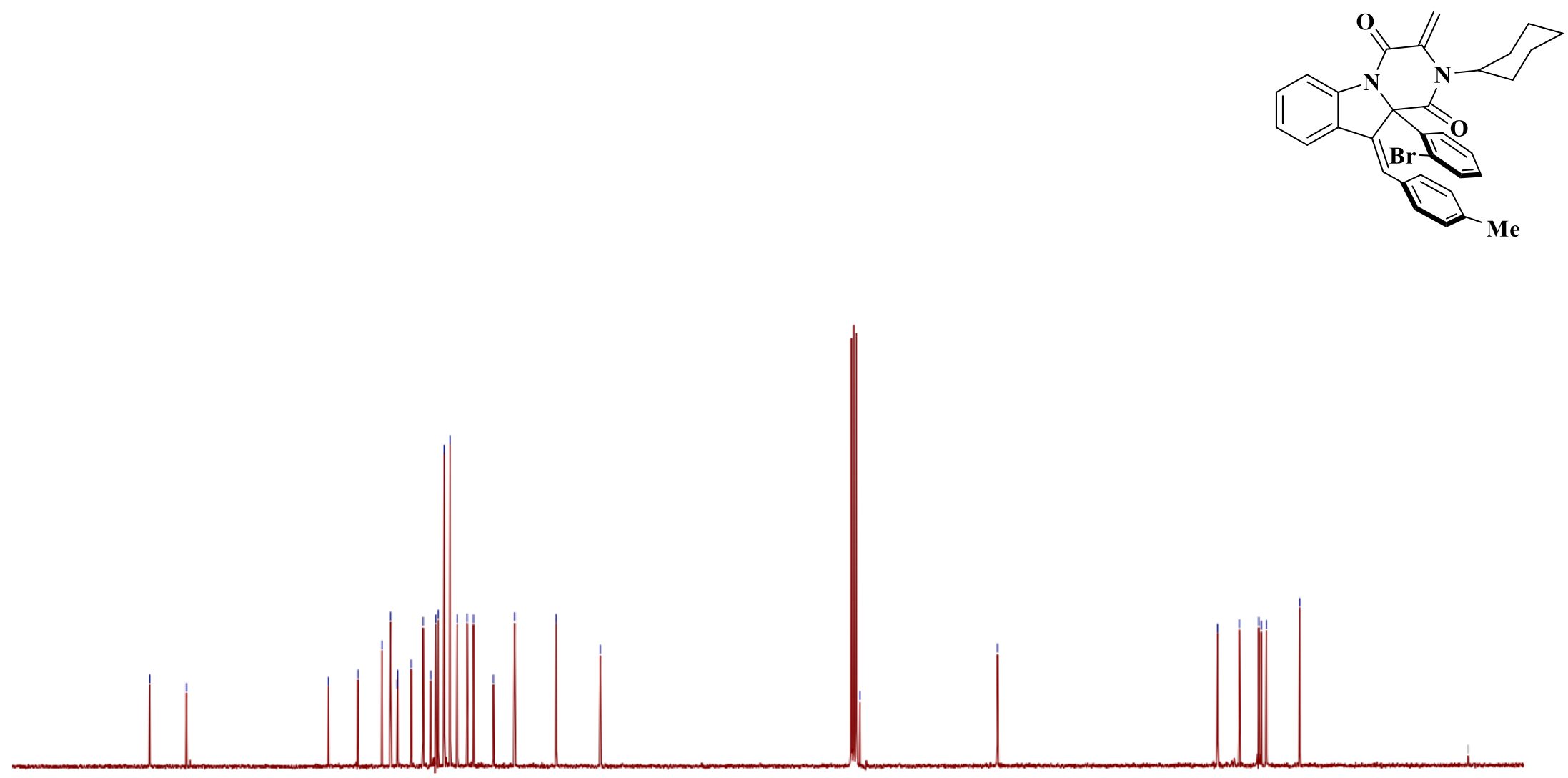

180 170 160

Figure S70. ${ }^{13} \mathrm{C} \mathrm{NMR}\left(100 \mathrm{MHz}, \mathrm{CDCl}_{3}\right) \mathbf{2 m}$ 


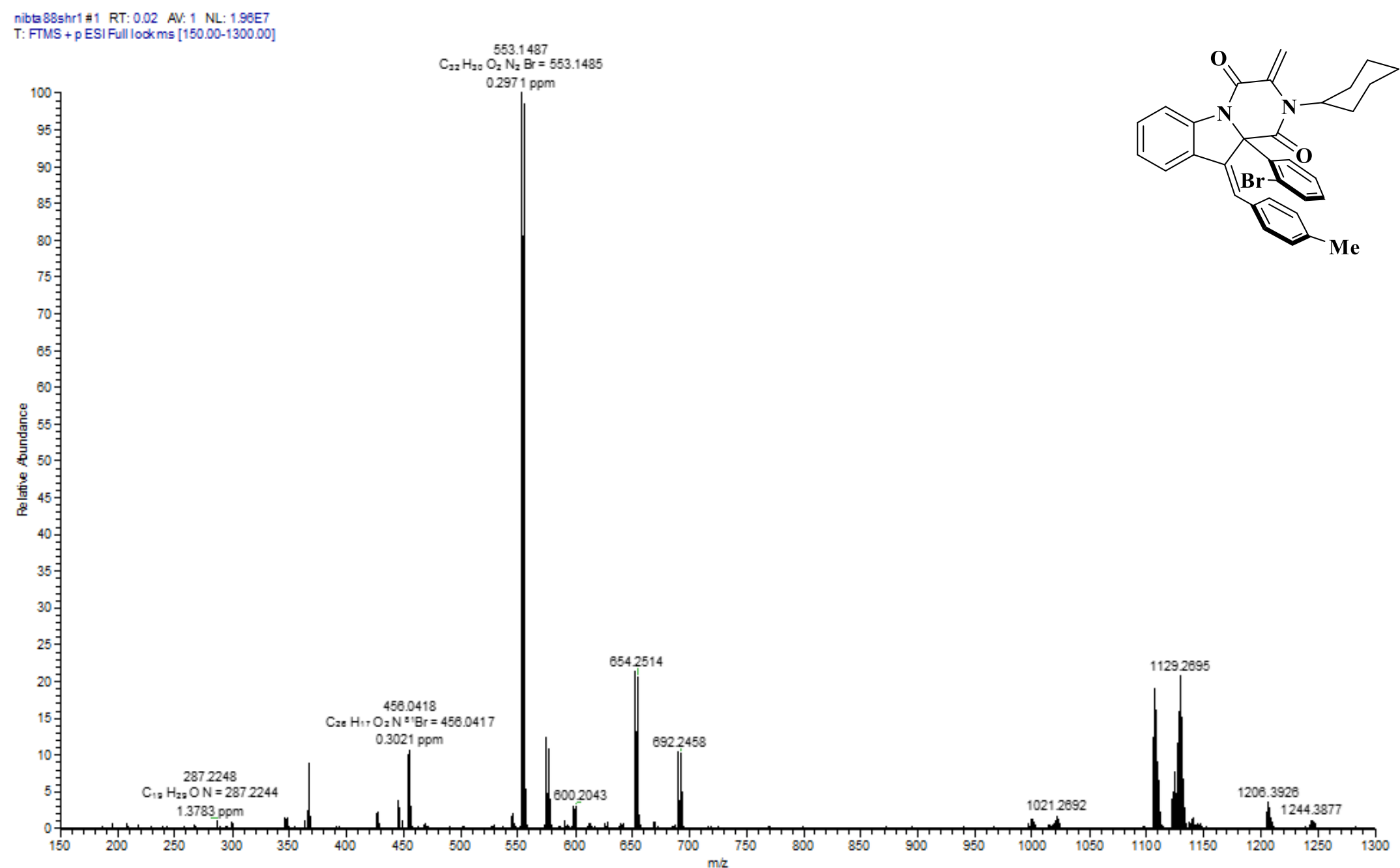

Figure S71. HRMS (ESI) 2m 


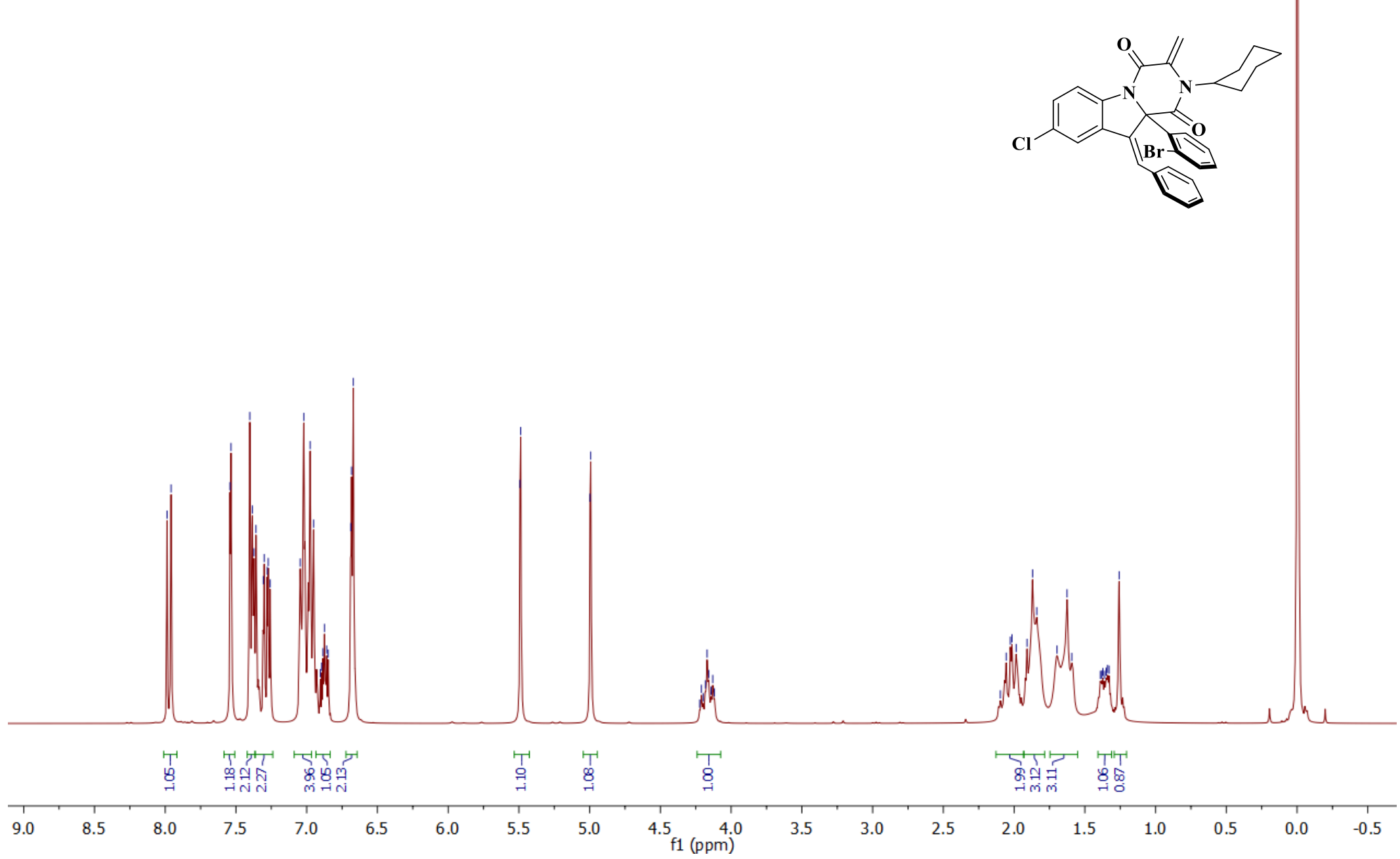

Figure $\mathrm{S} 72 .{ }^{1} \mathrm{H} \mathrm{NMR}\left(300 \mathrm{MHz}, \mathrm{CDCl}_{3}\right)$ 2n 

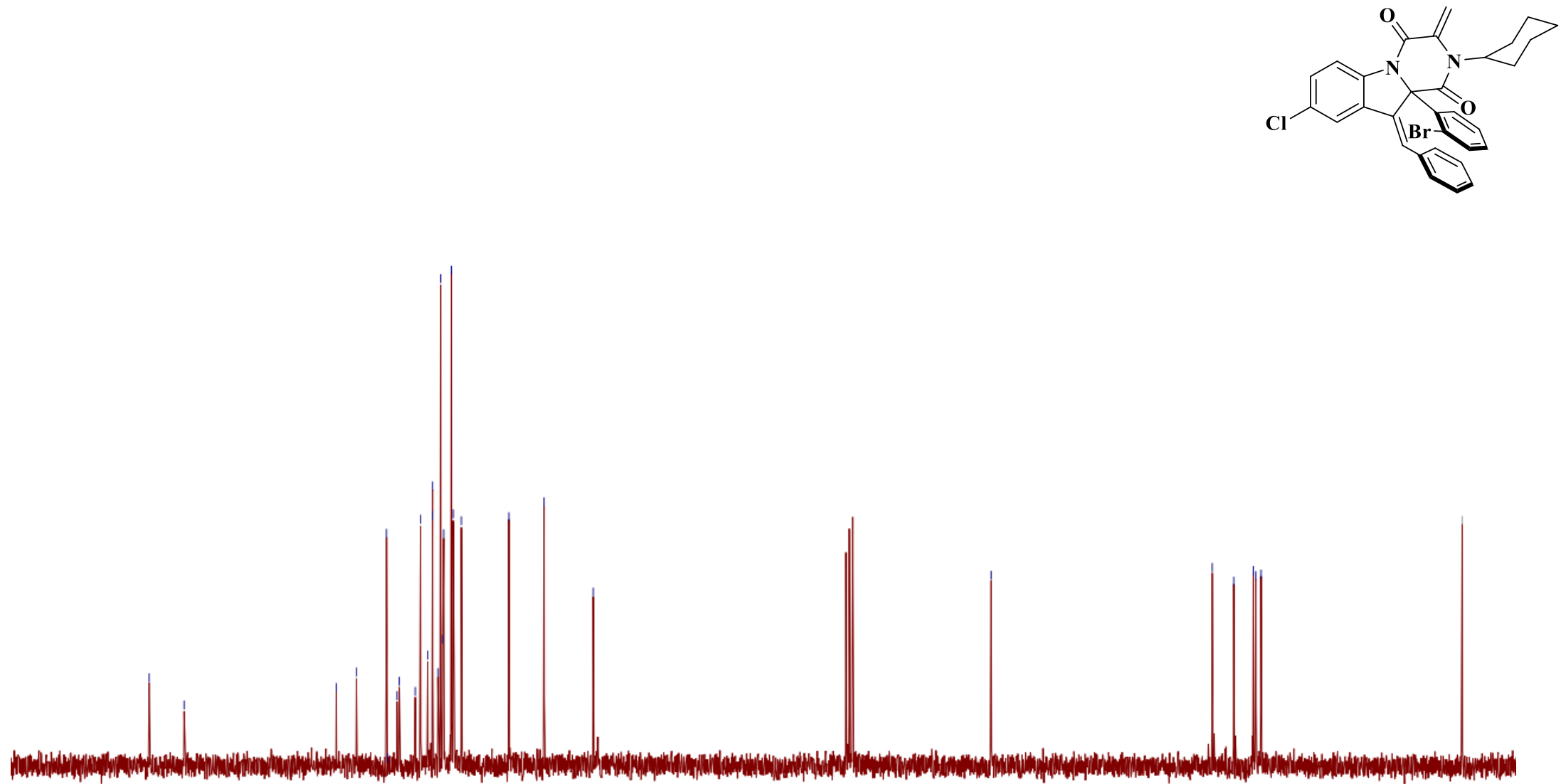

180

$170 \quad 160$

150

140

130

120

$110 \quad 100$

$\mathrm{f}^{90}(\mathrm{ppm}){ }^{80}$

70

60

50

40

30

20

10

Figure S73. ${ }^{13} \mathrm{C} \mathrm{NMR}\left(75 \mathrm{MHz}, \mathrm{CDCl}_{3}\right)$ 2n 


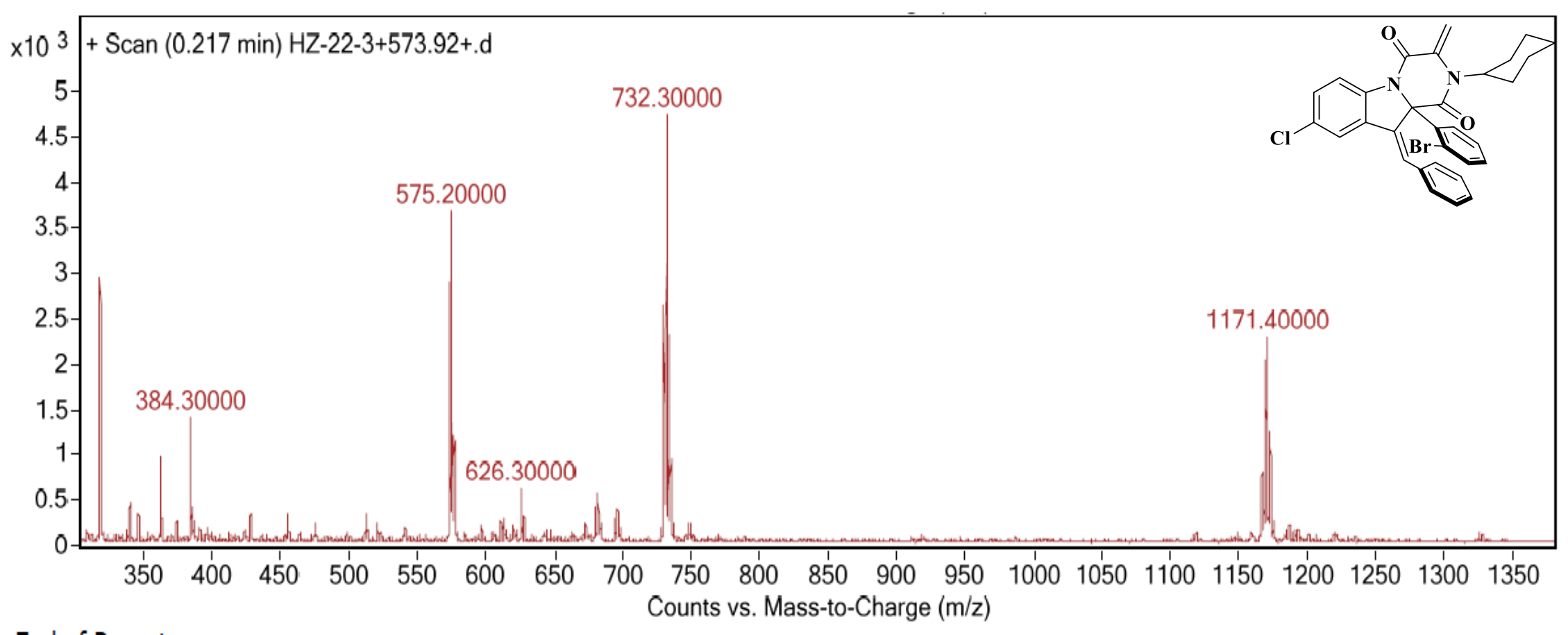

Figure S74. MS (ESI) 2n 


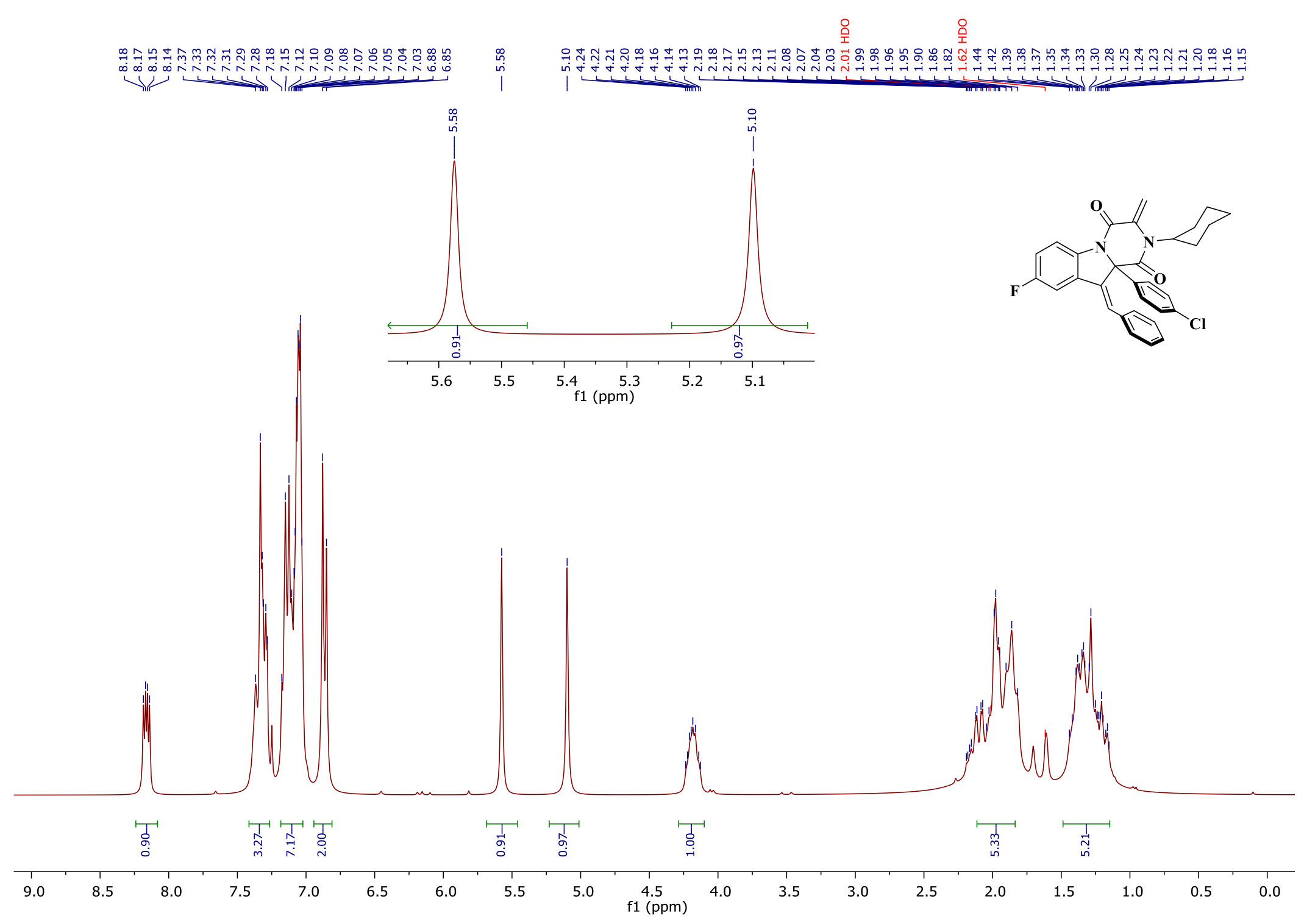

Figure S75. ${ }^{1} \mathrm{H} \mathrm{NMR}\left(300 \mathrm{MHz}, \mathrm{CDCl}_{3}\right) 2 \mathbf{2}$ 

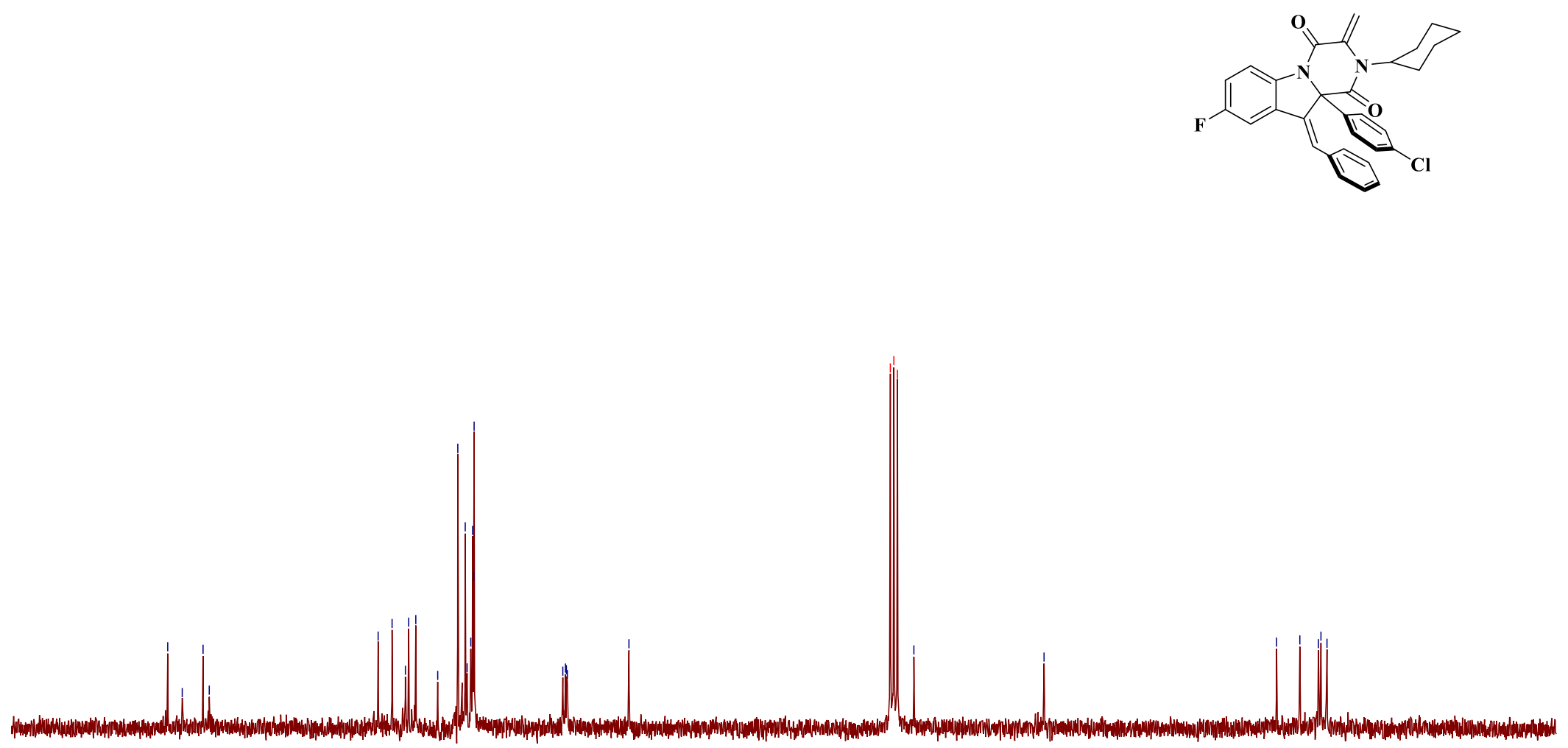

Figure S76. ${ }^{13} \mathrm{C} \mathrm{NMR}\left(75 \mathrm{MHz}, \mathrm{CDCl}_{3}\right) \mathbf{2 o}$ 


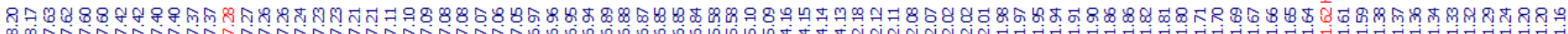
作

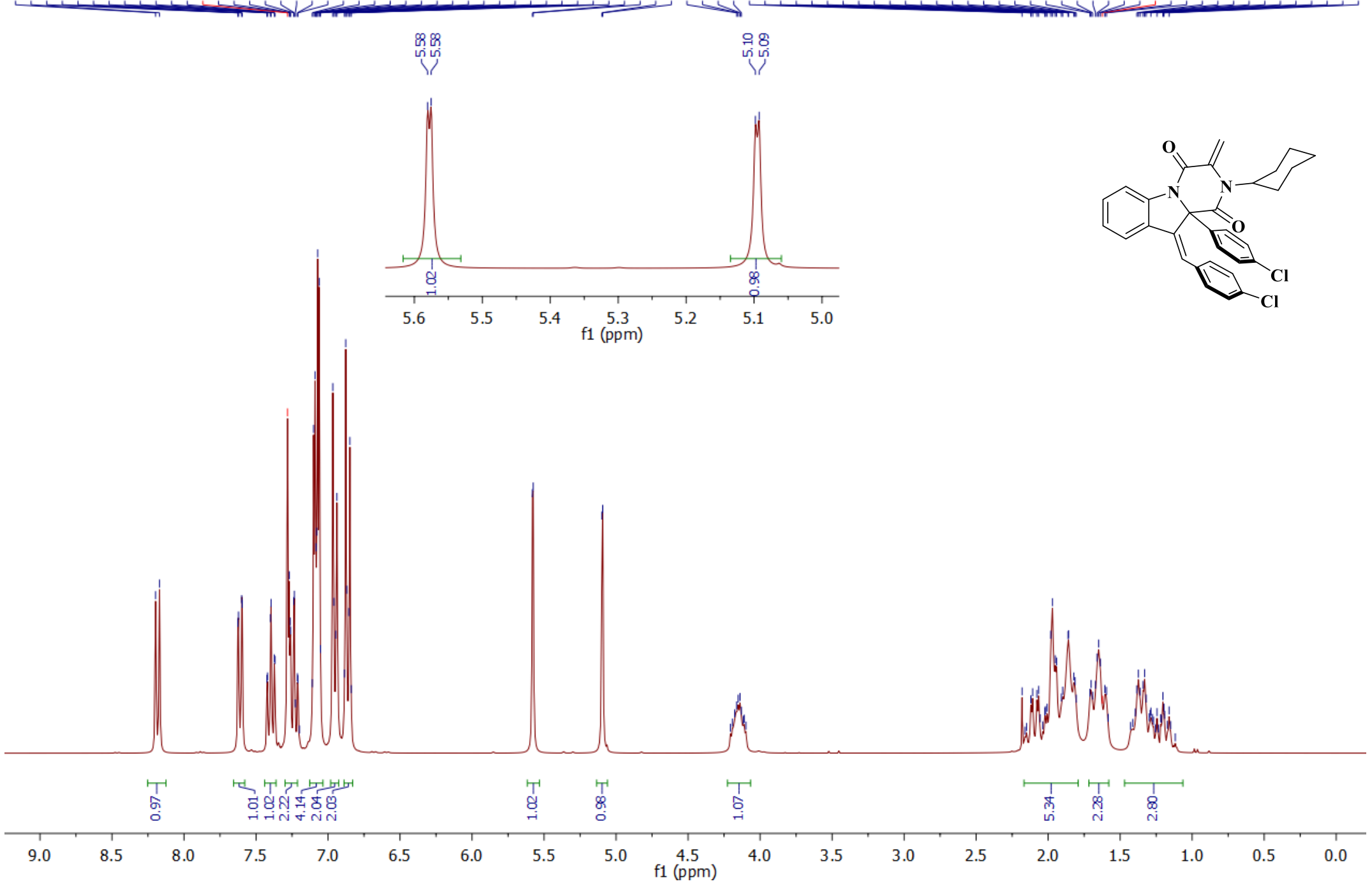

Figure $\mathrm{S} 77 .{ }^{1} \mathrm{H} \mathrm{NMR}\left(300 \mathrm{MHz}, \mathrm{CDCl}_{3}\right) \mathbf{2 p}$ 

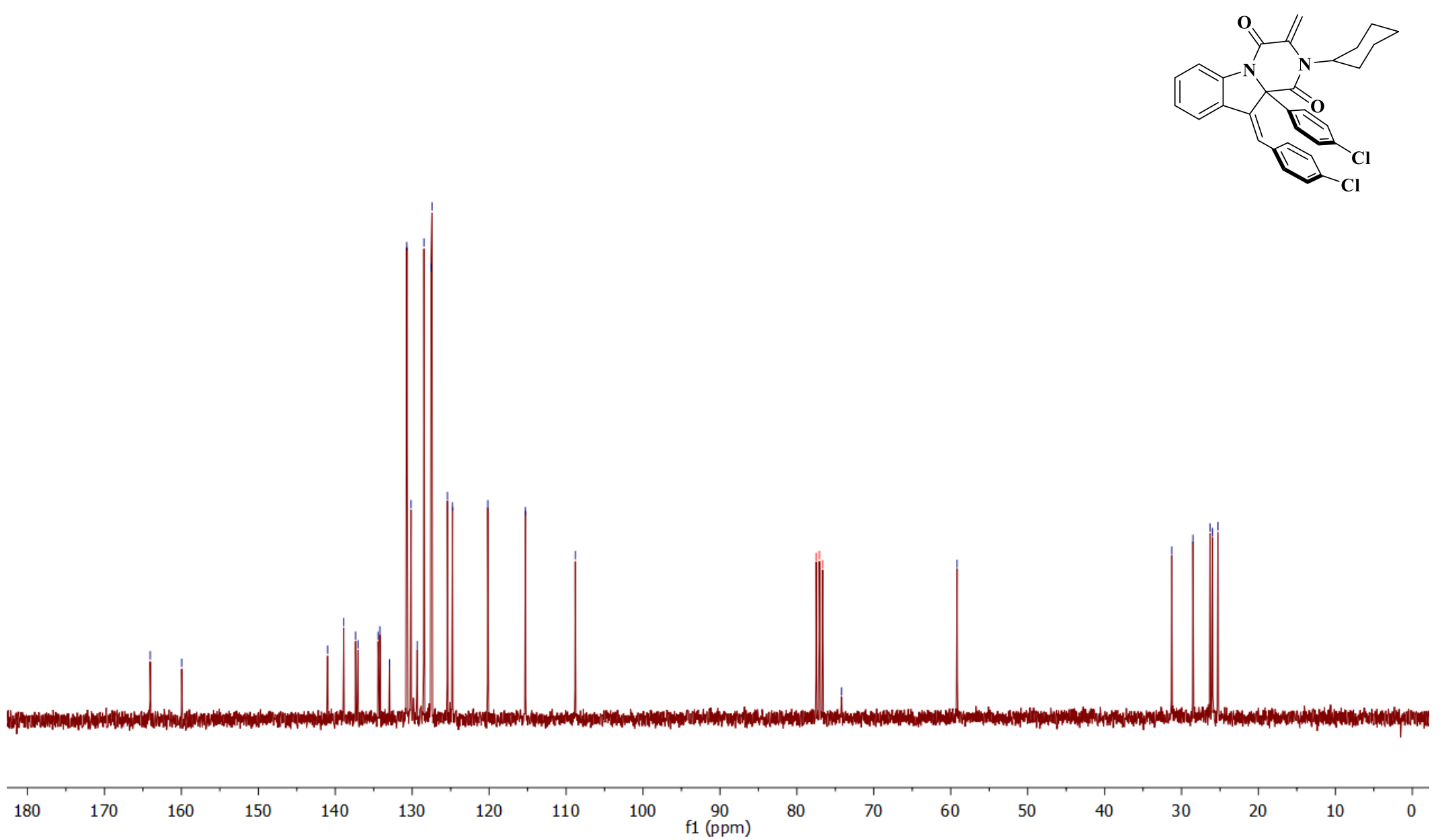

Figure $\mathrm{S} 78 .{ }^{13} \mathrm{C} \mathrm{NMR}\left(75 \mathrm{MHz}, \mathrm{CDCl}_{3}\right) \mathbf{2 p}$ 


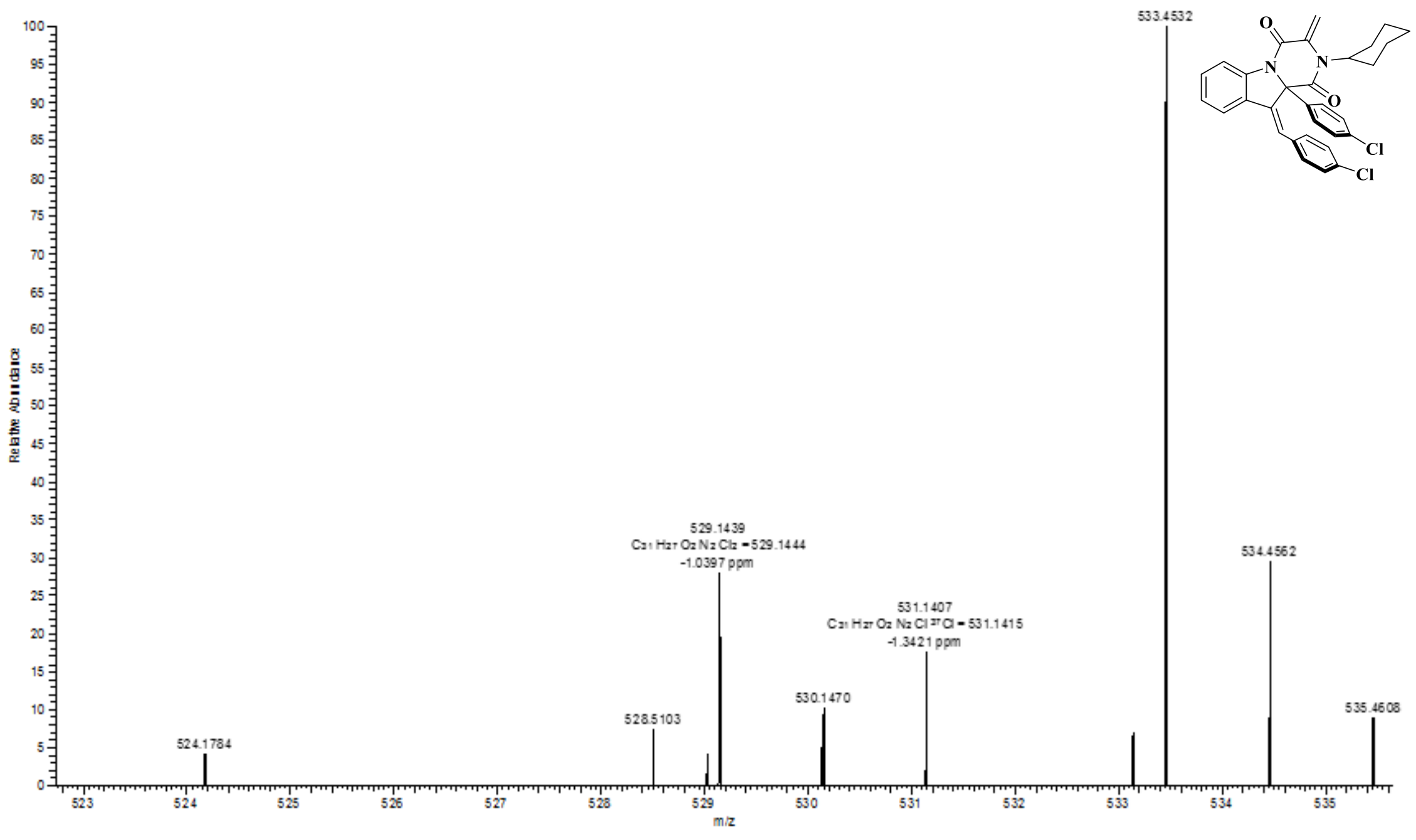

Figure S79. HRMS (ESI) 2p 


\section{${ }^{1} \mathrm{H}$ NMR, ${ }^{13} \mathrm{C}$ NMR and HRMS Spectra:}

8.

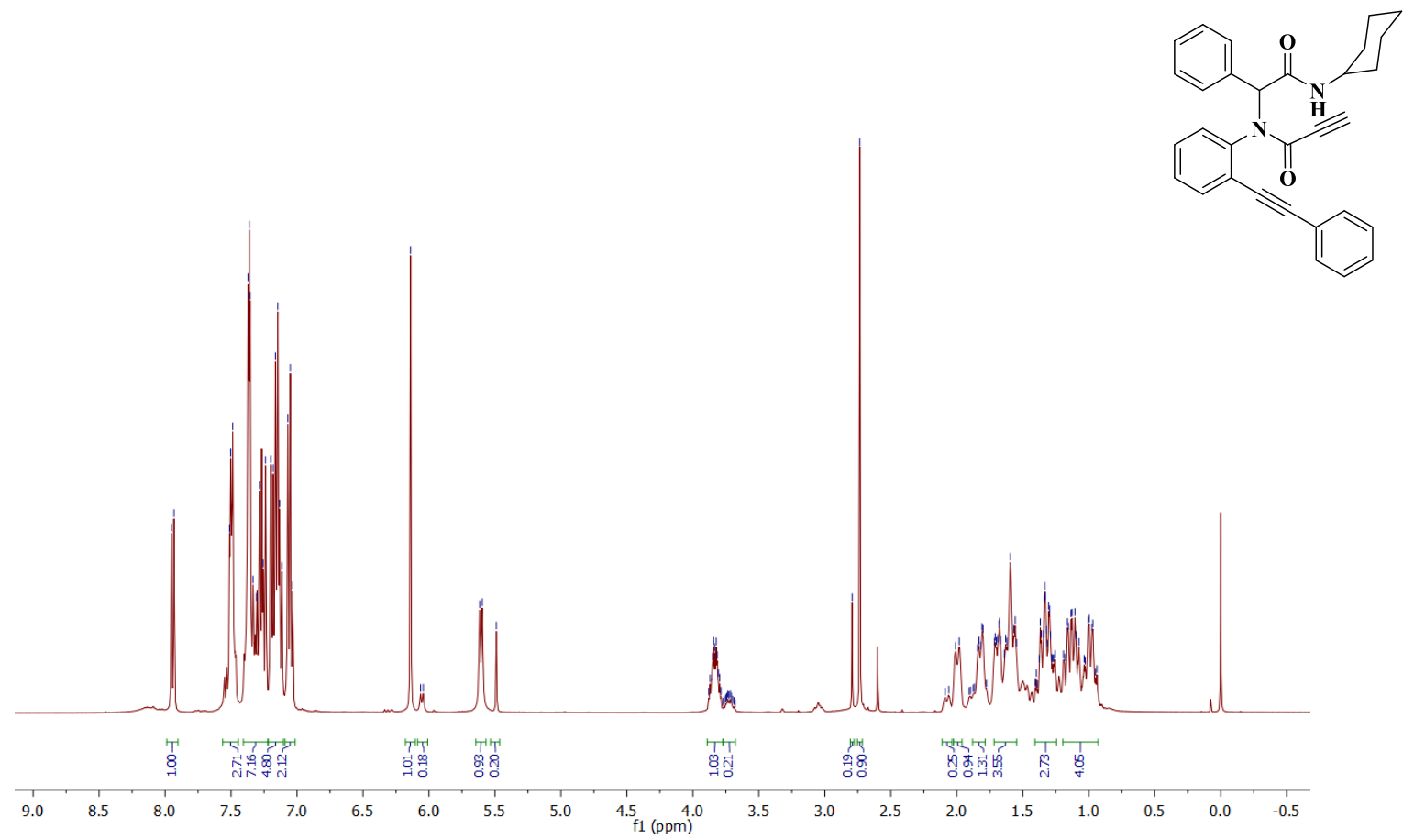

Figure S80. ${ }^{1} \mathrm{H}$ NMR- compound $\mathbf{3 a}$

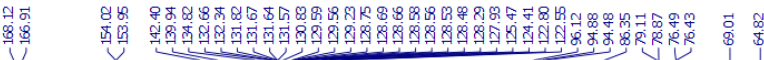

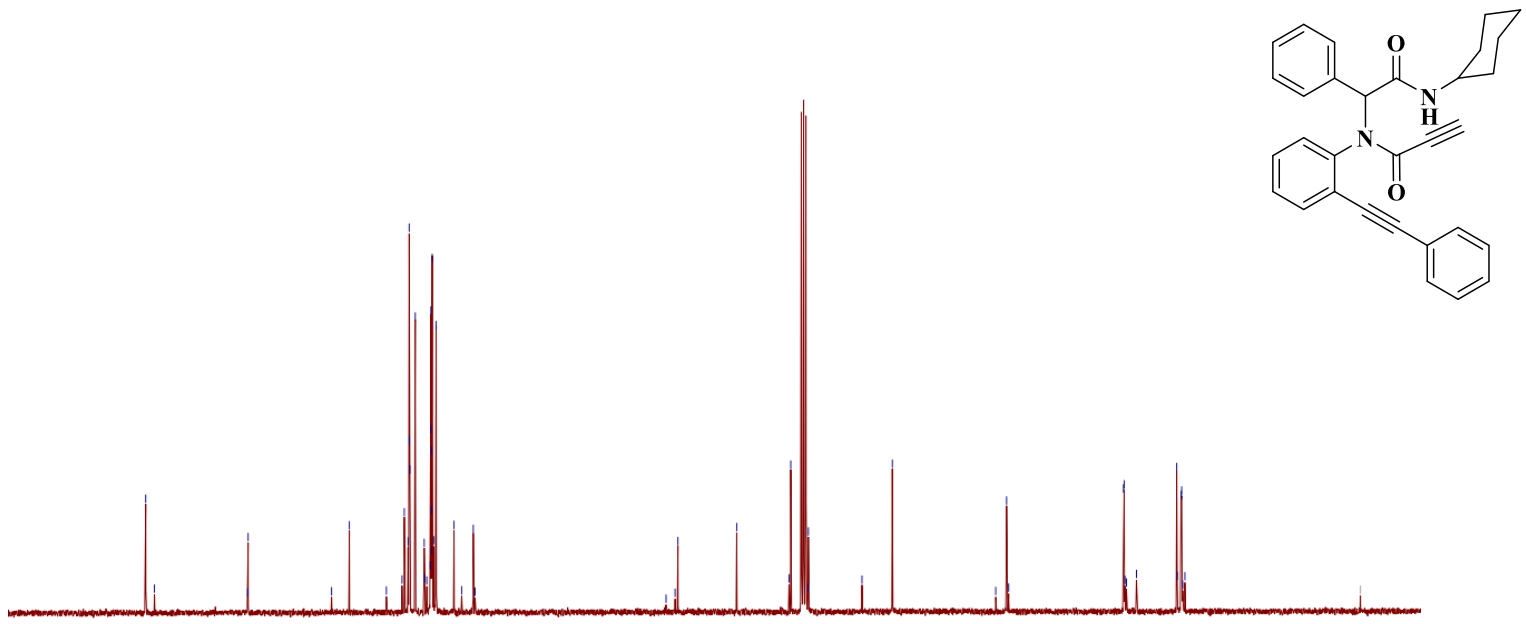

Figure S81. ${ }^{13} \mathrm{C}$ NMR- compound 3a 


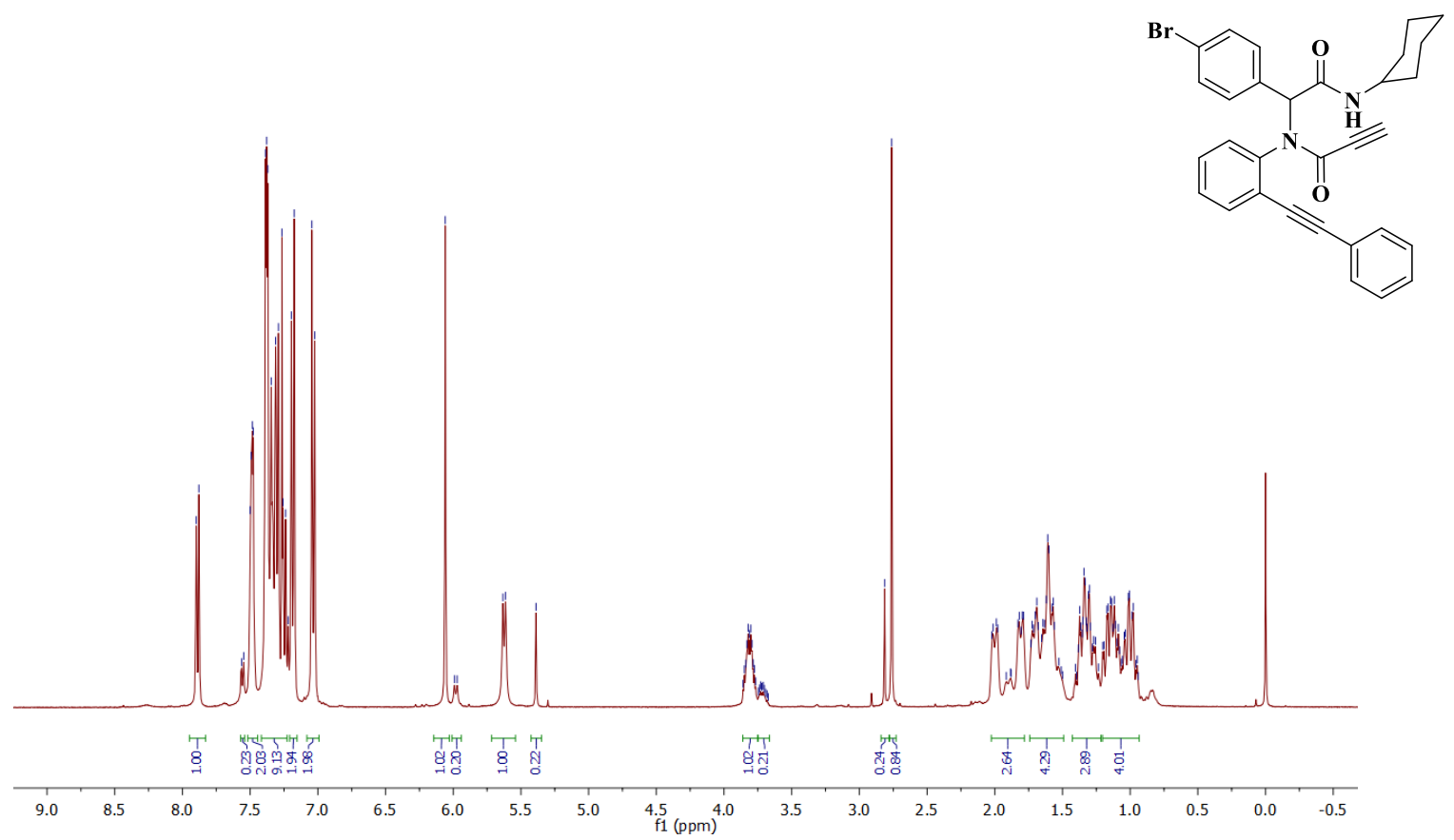

Figure S82. ${ }^{1} \mathrm{H}$ NMR- compound $\mathbf{3 b}$
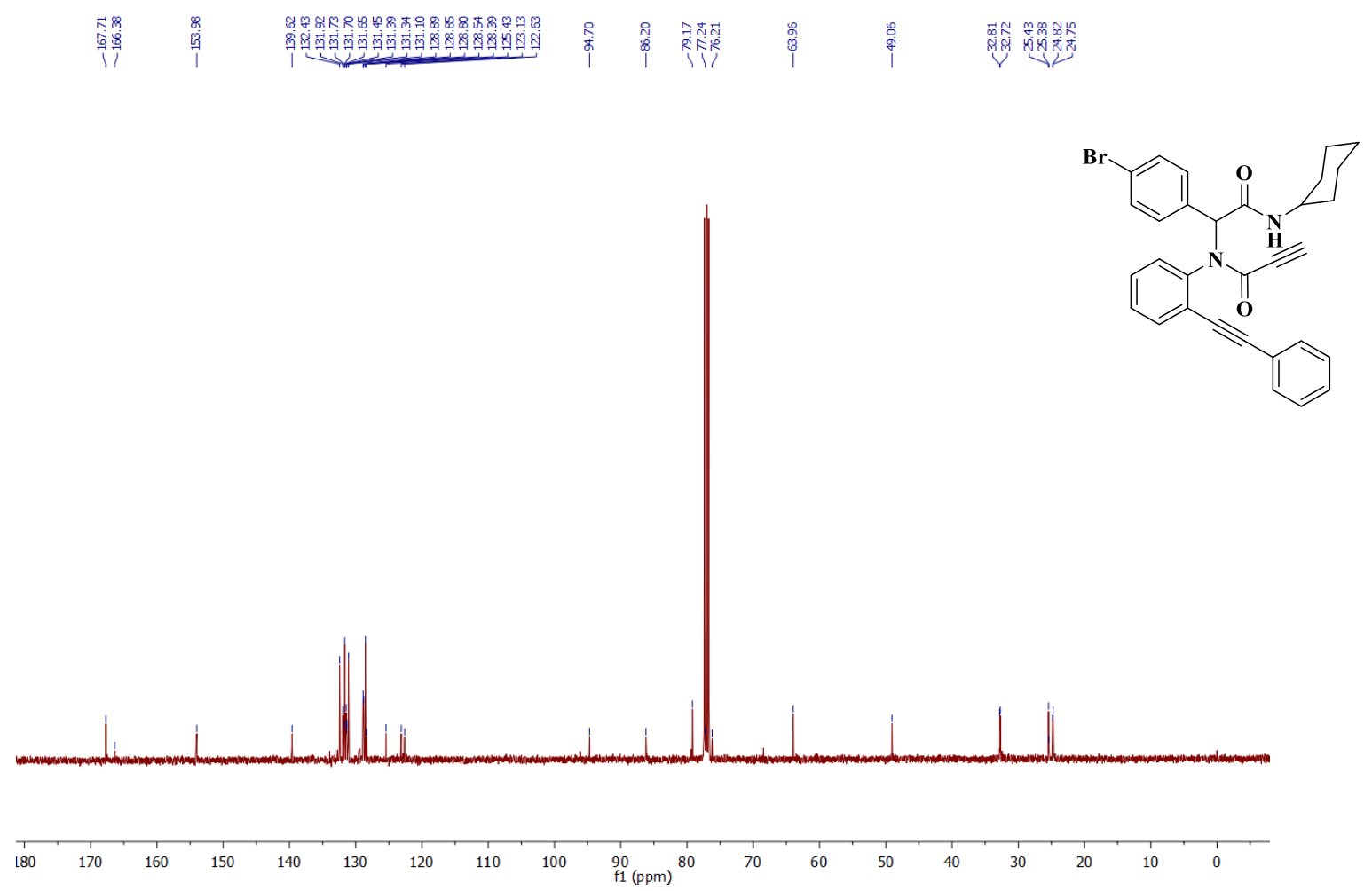

Figure S83. ${ }^{13} \mathrm{C}$ NMR- compound $\mathbf{3 b}$ 


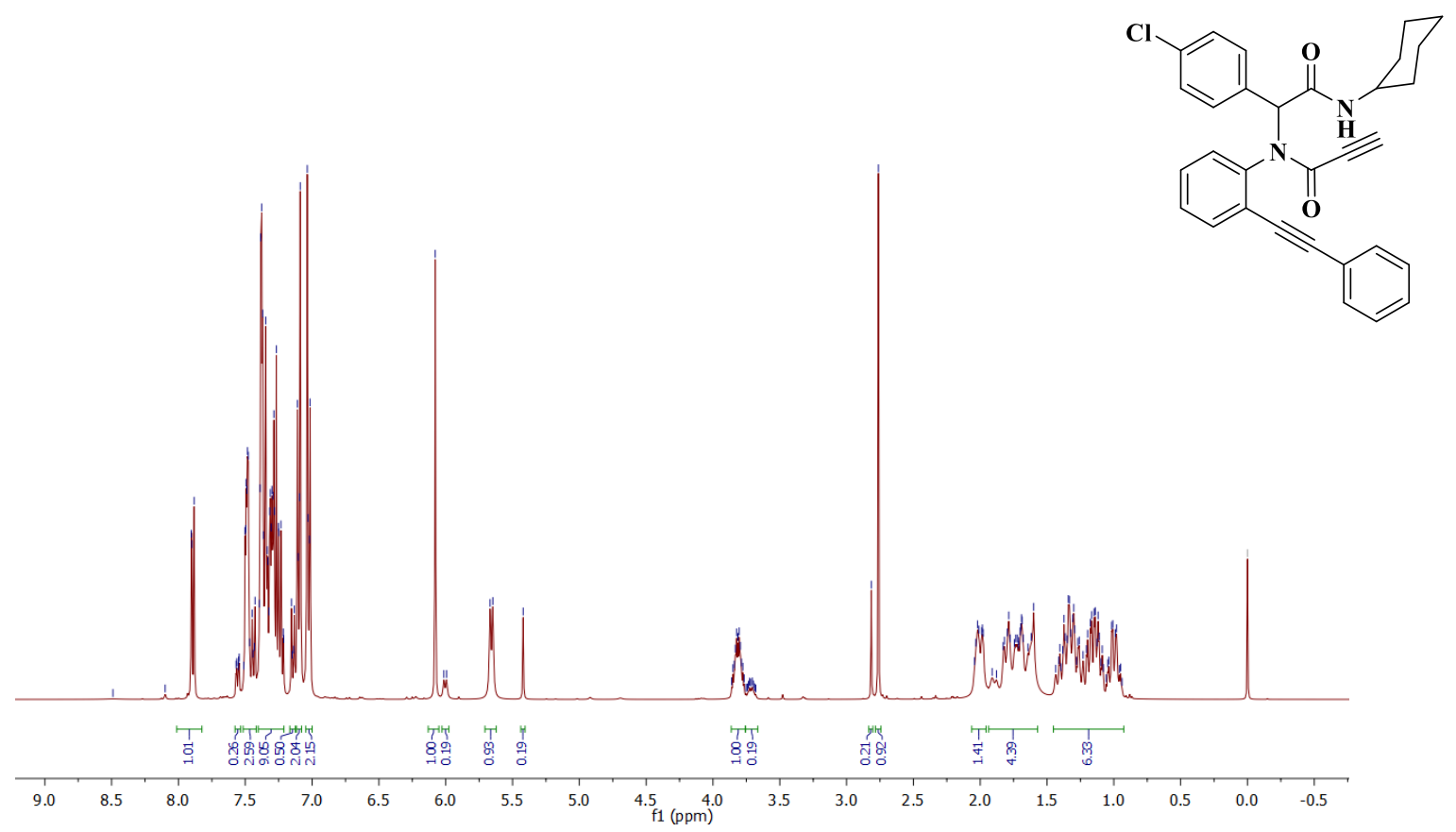

Figure S84. ${ }^{1} \mathrm{H}$ NMR- compound 3c

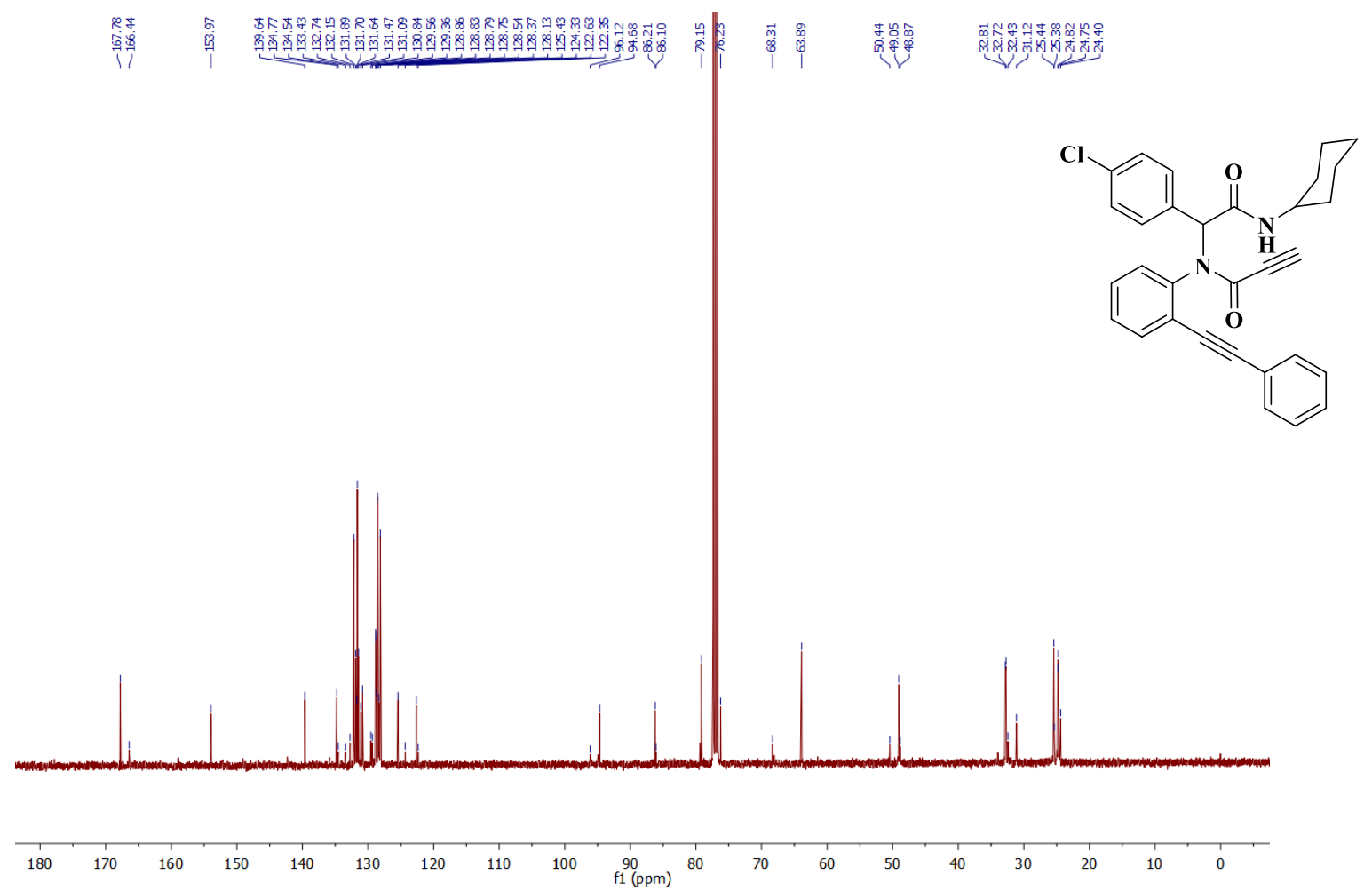

Figure S85. ${ }^{13} \mathrm{C}$ NMR- compound 3c 


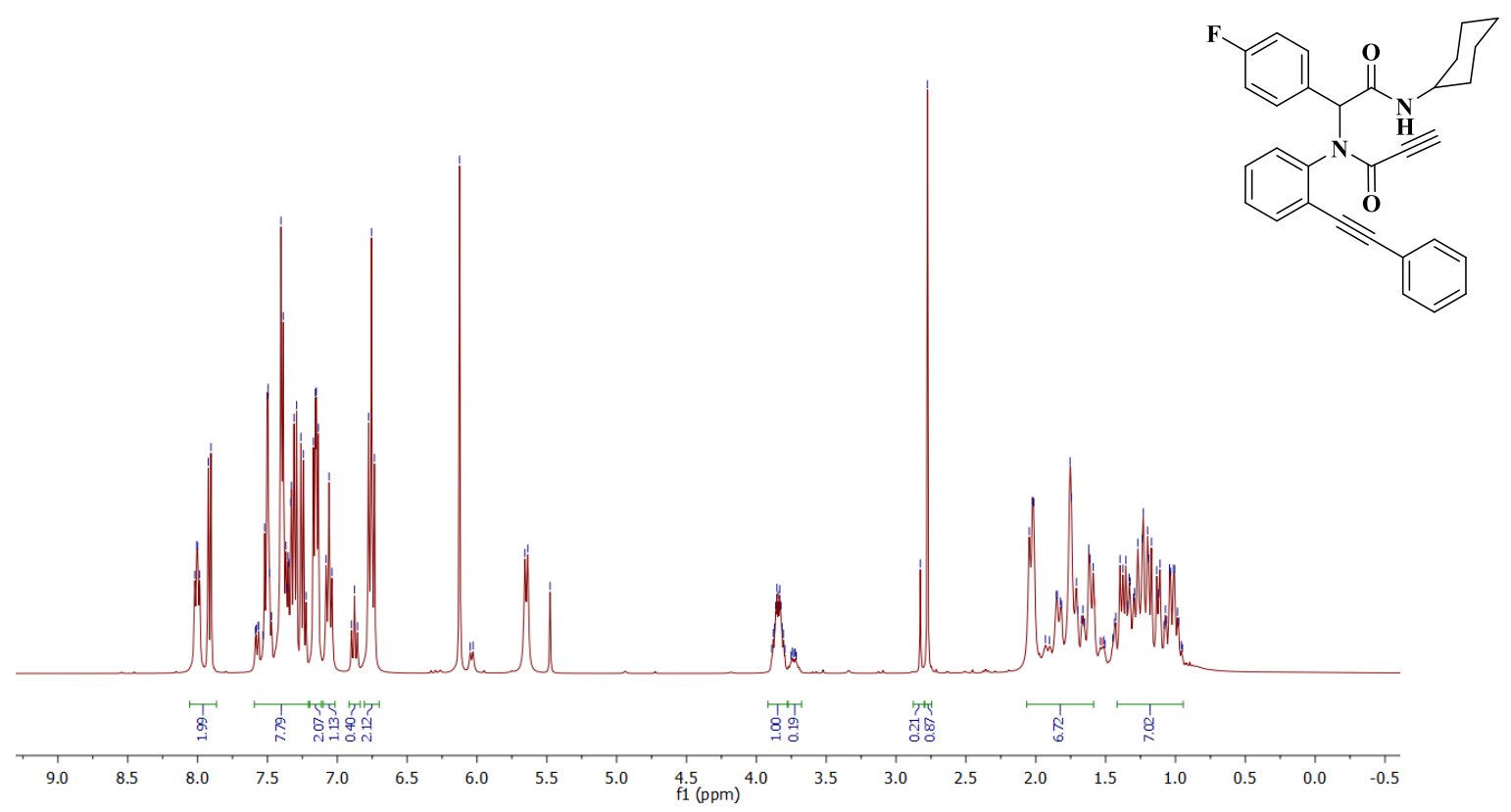

Figure S86. ${ }^{1} \mathrm{H}$ NMR- compound $3 \mathbf{d}$

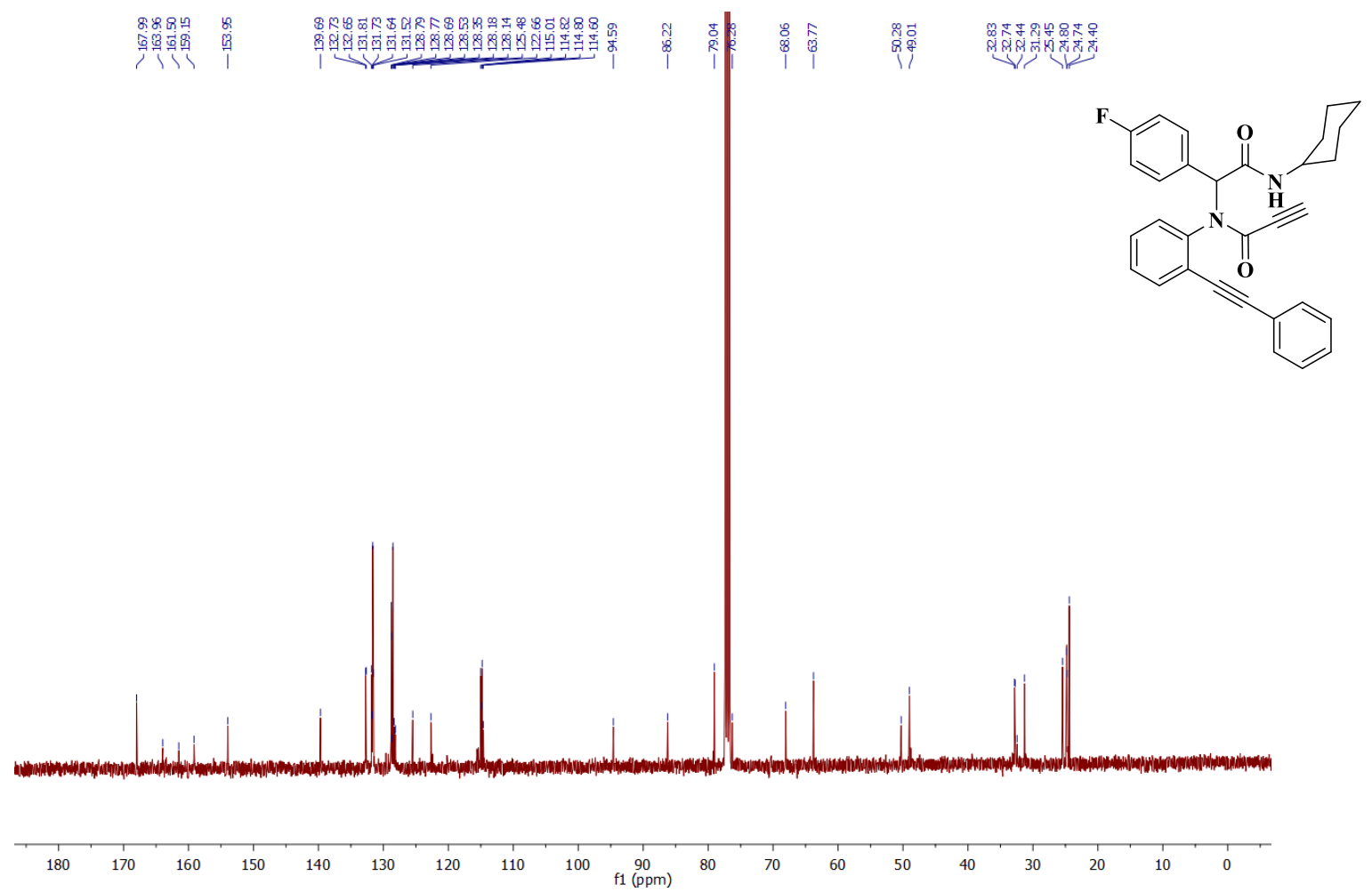

Figure S87. ${ }^{13}$ C NMR- compound 3d 


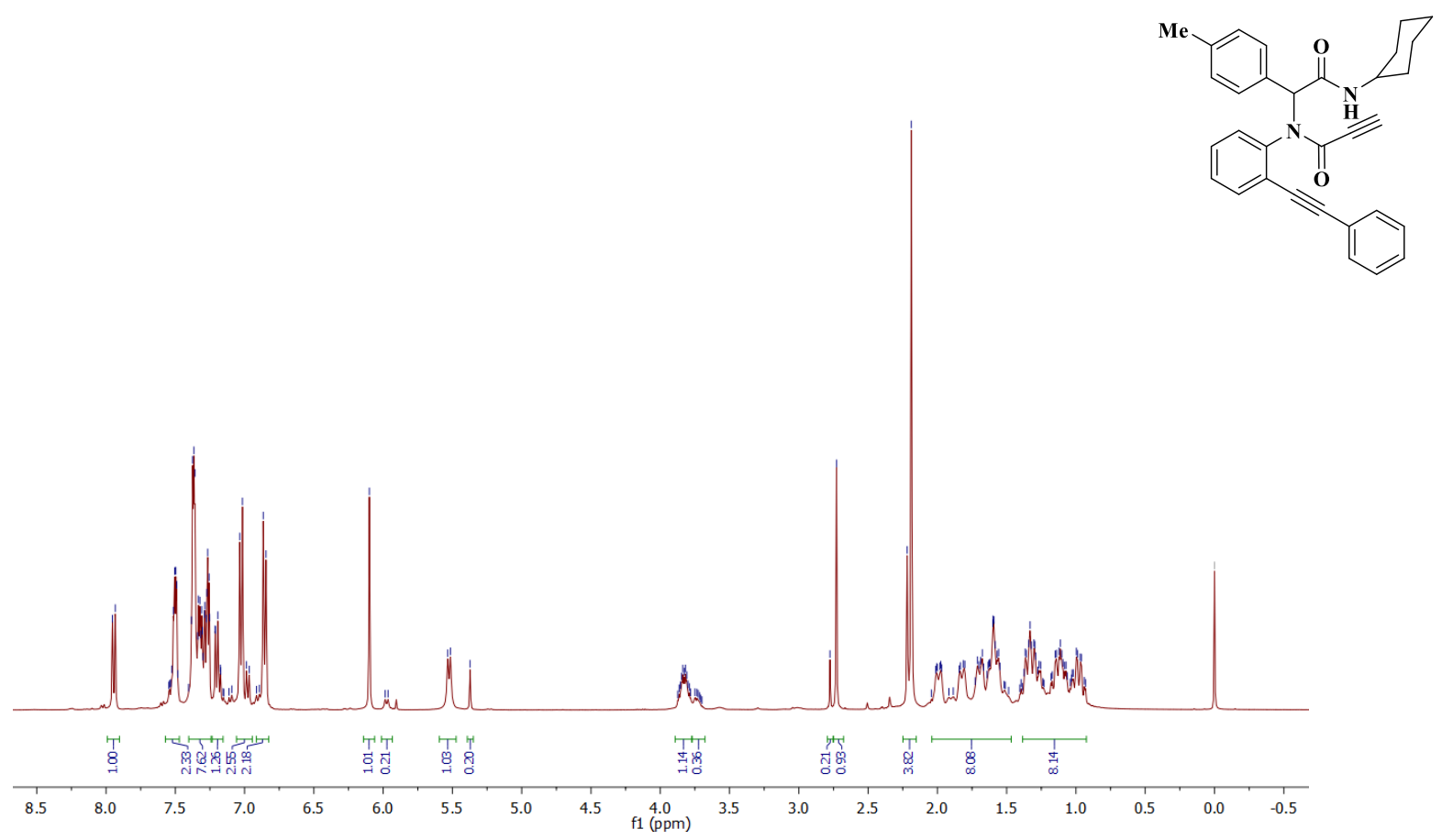

Figure S88. ${ }^{1} \mathrm{H}$ NMR- compound $3 \mathbf{e}$

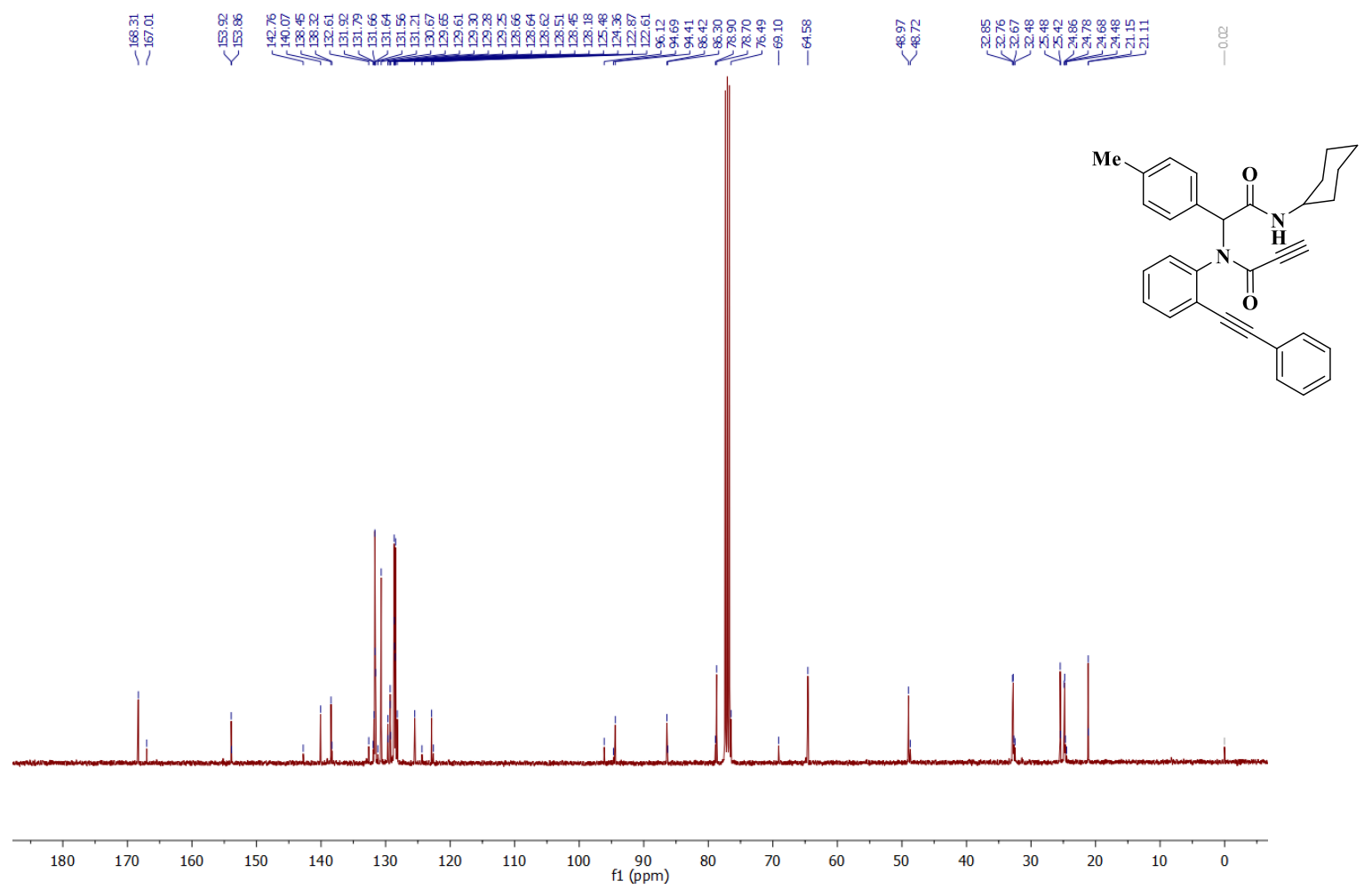

Figure S89. ${ }^{13} \mathrm{C}$ NMR- compound $\mathbf{3 e}$ 


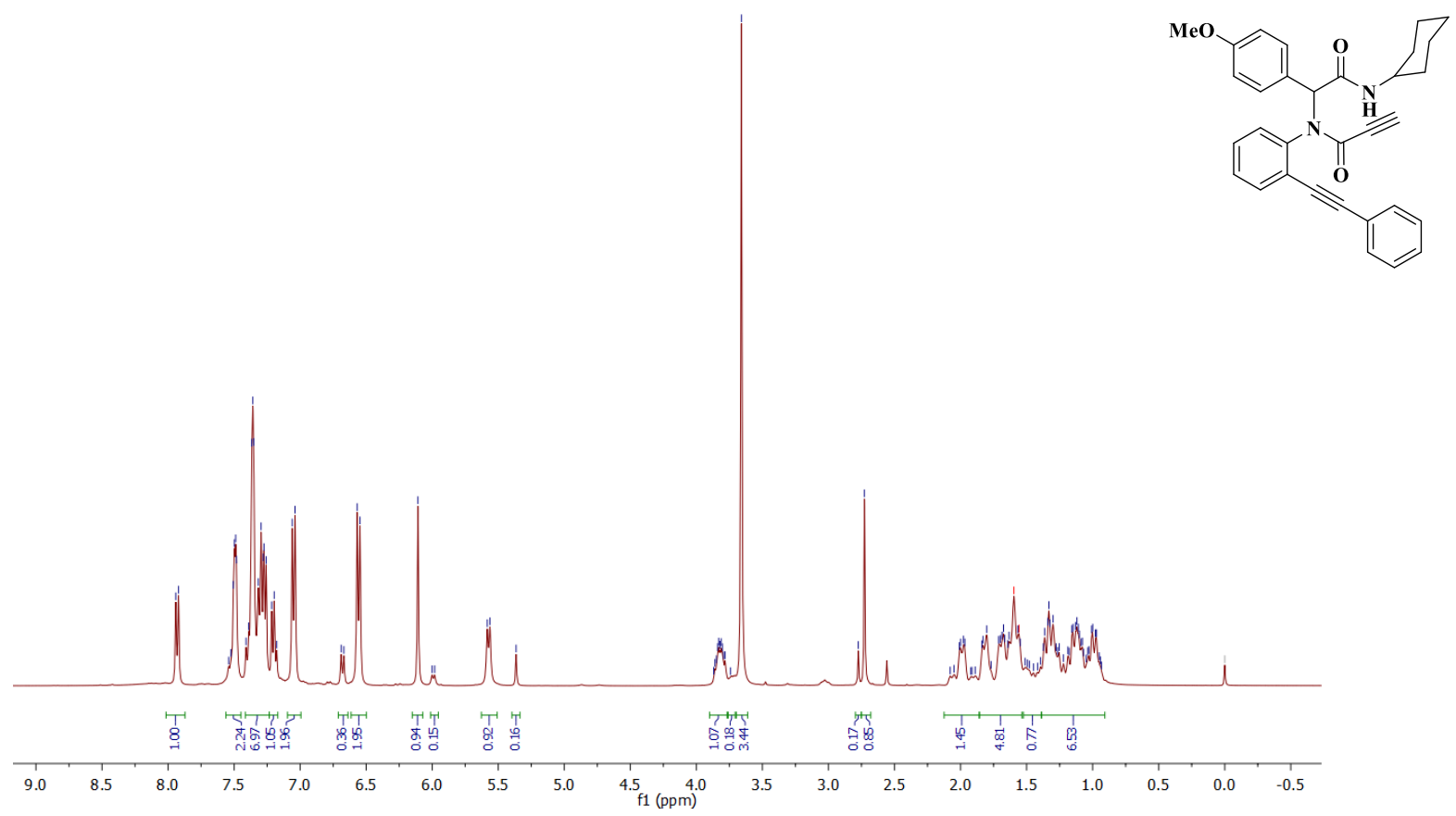

Figure S90. ${ }^{1} \mathrm{H}$ NMR- compound $\mathbf{3 f}$

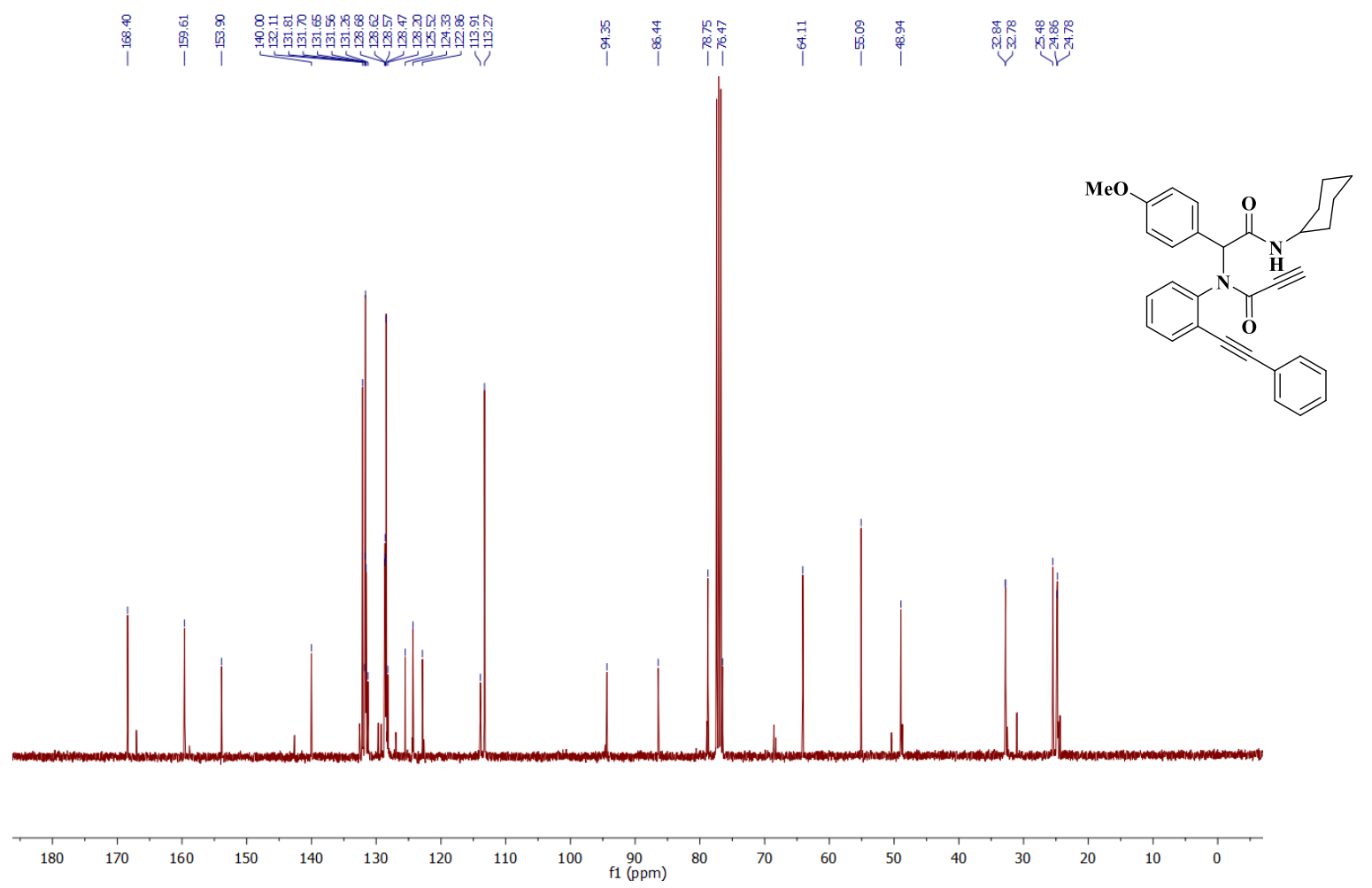

Figure S91. ${ }^{13} \mathrm{C}$ NMR- compound $\mathbf{3 f}$ 


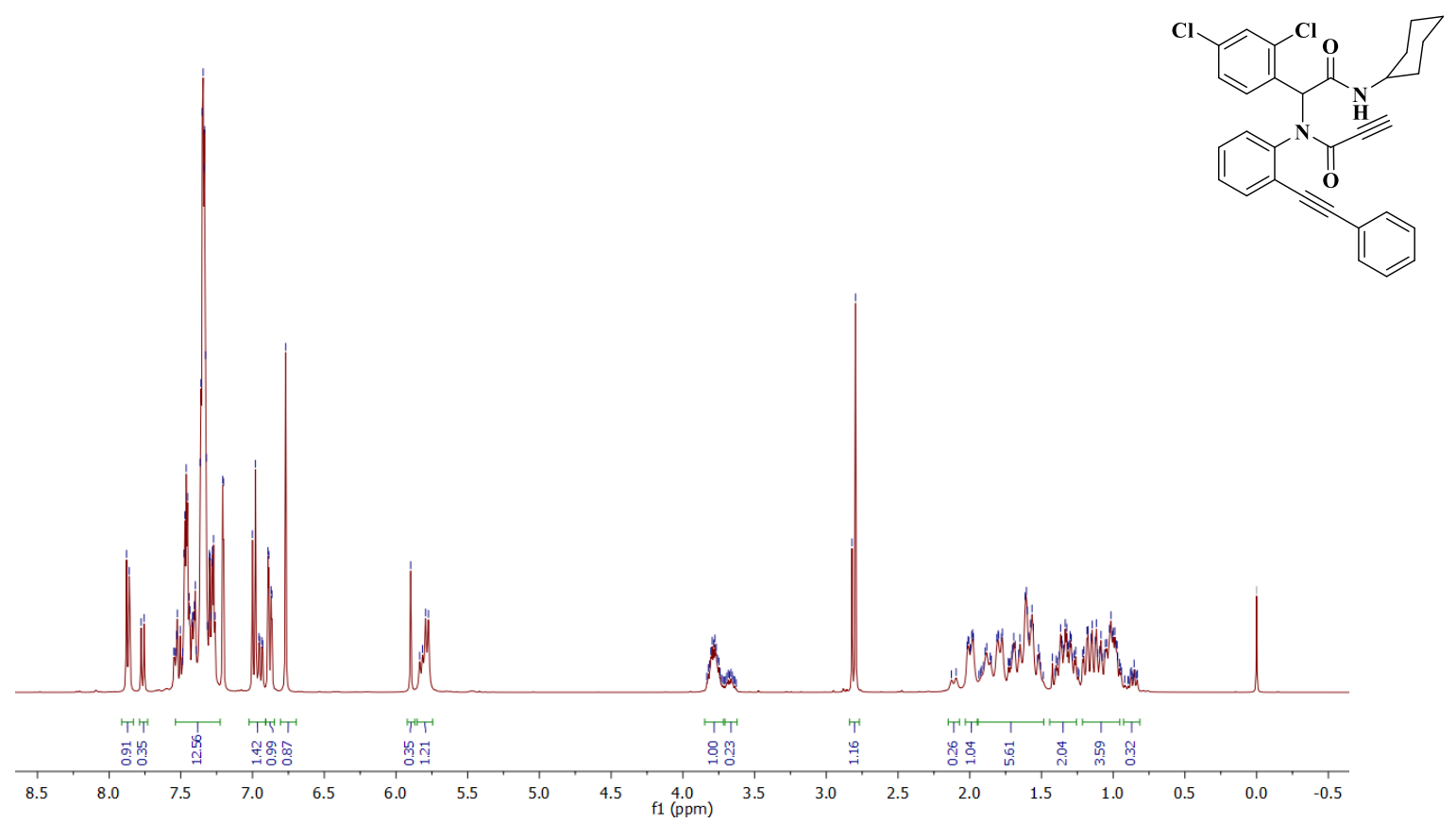

Figure S92. ${ }^{1} \mathrm{H}$ NMR- compound 3g

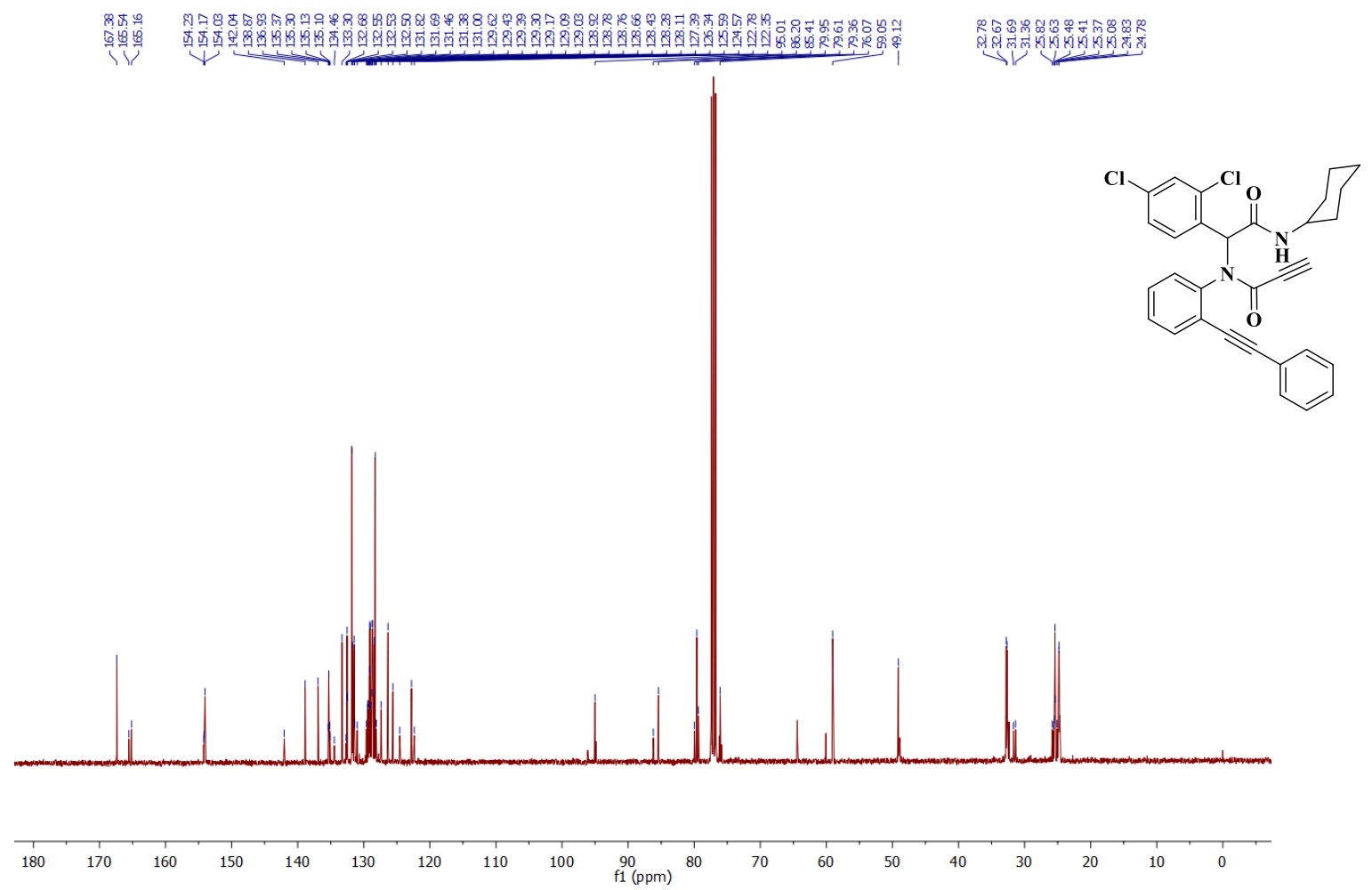

Figure S93. ${ }^{13} \mathrm{C}$ NMR- compound 3g 


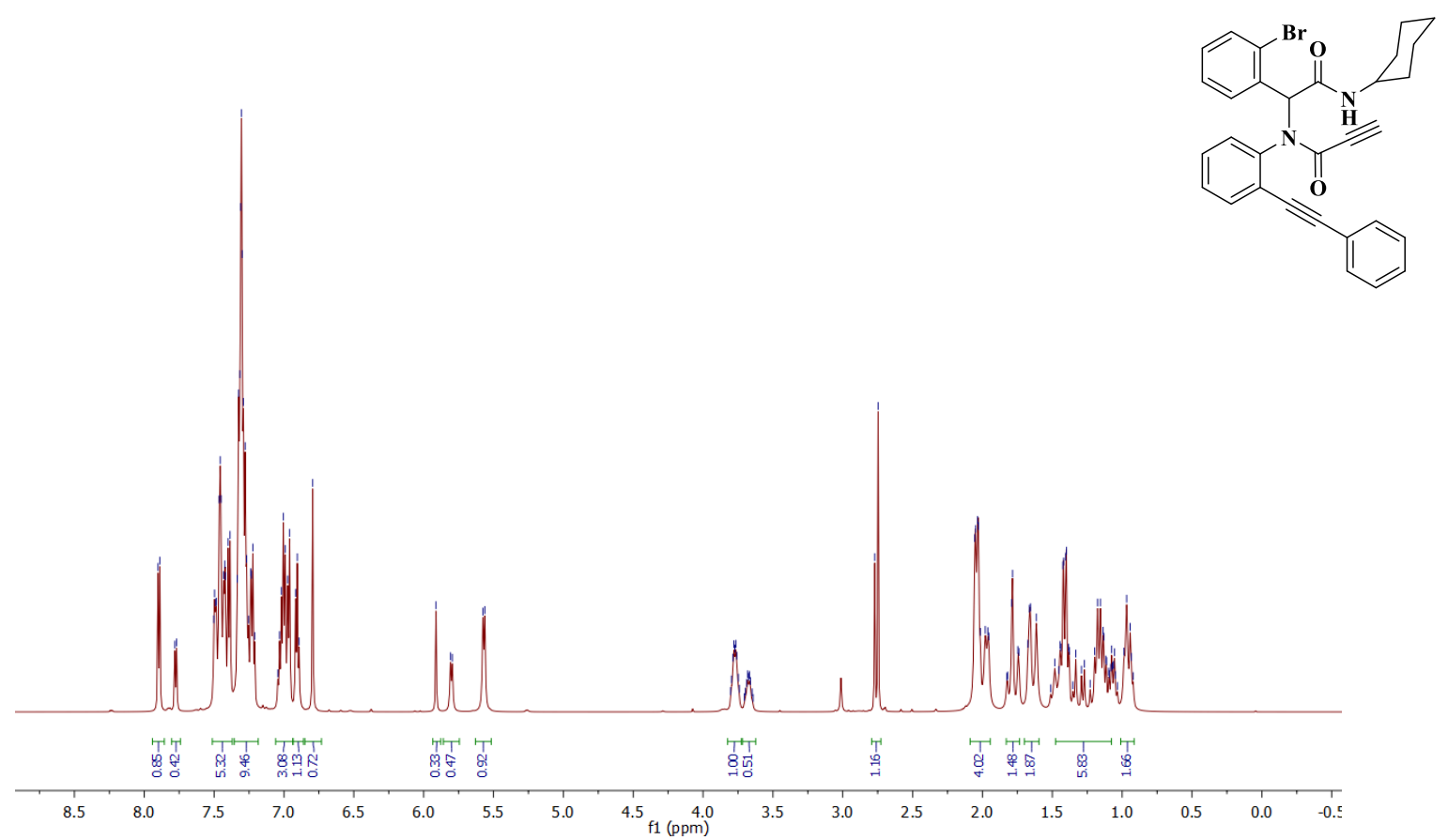

Figure S94. ${ }^{1} \mathrm{H}$ NMR- compound $3 \mathbf{h}$

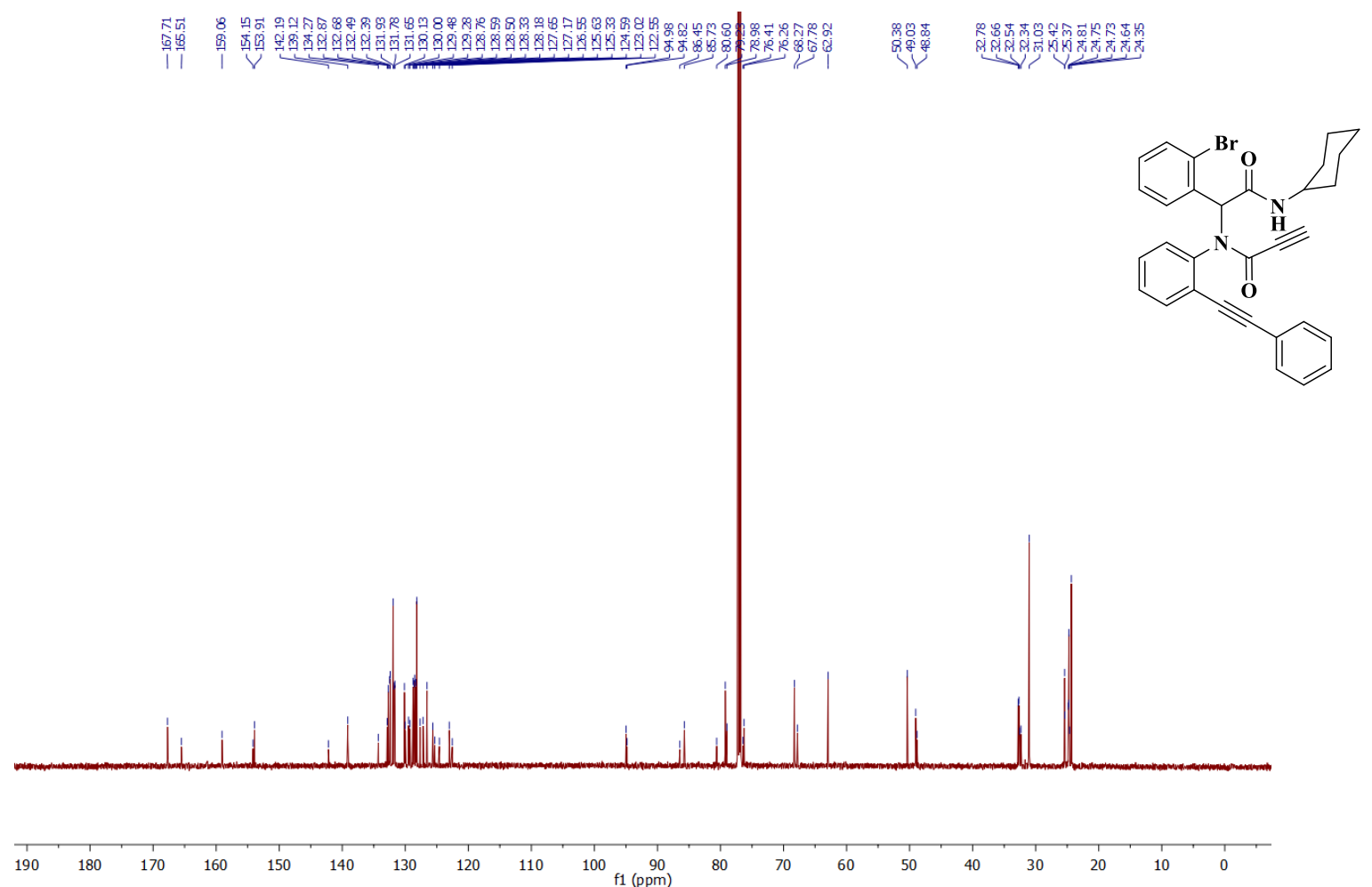

Figure S95. ${ }^{13} \mathrm{C}$ NMR- compound $3 \mathbf{h}$ 


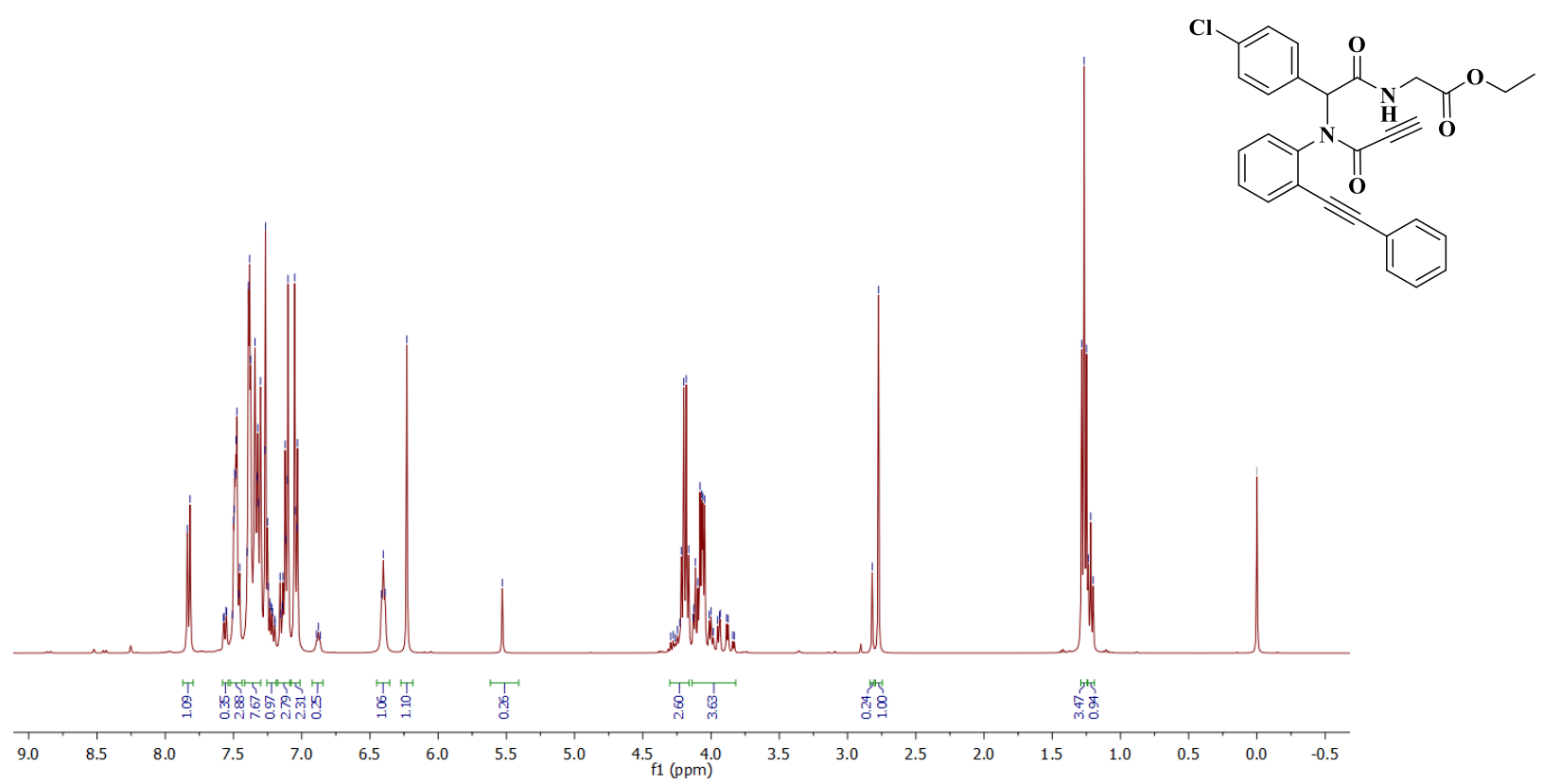

Figure S96. ${ }^{1} \mathrm{H}$ NMR- compound 3i

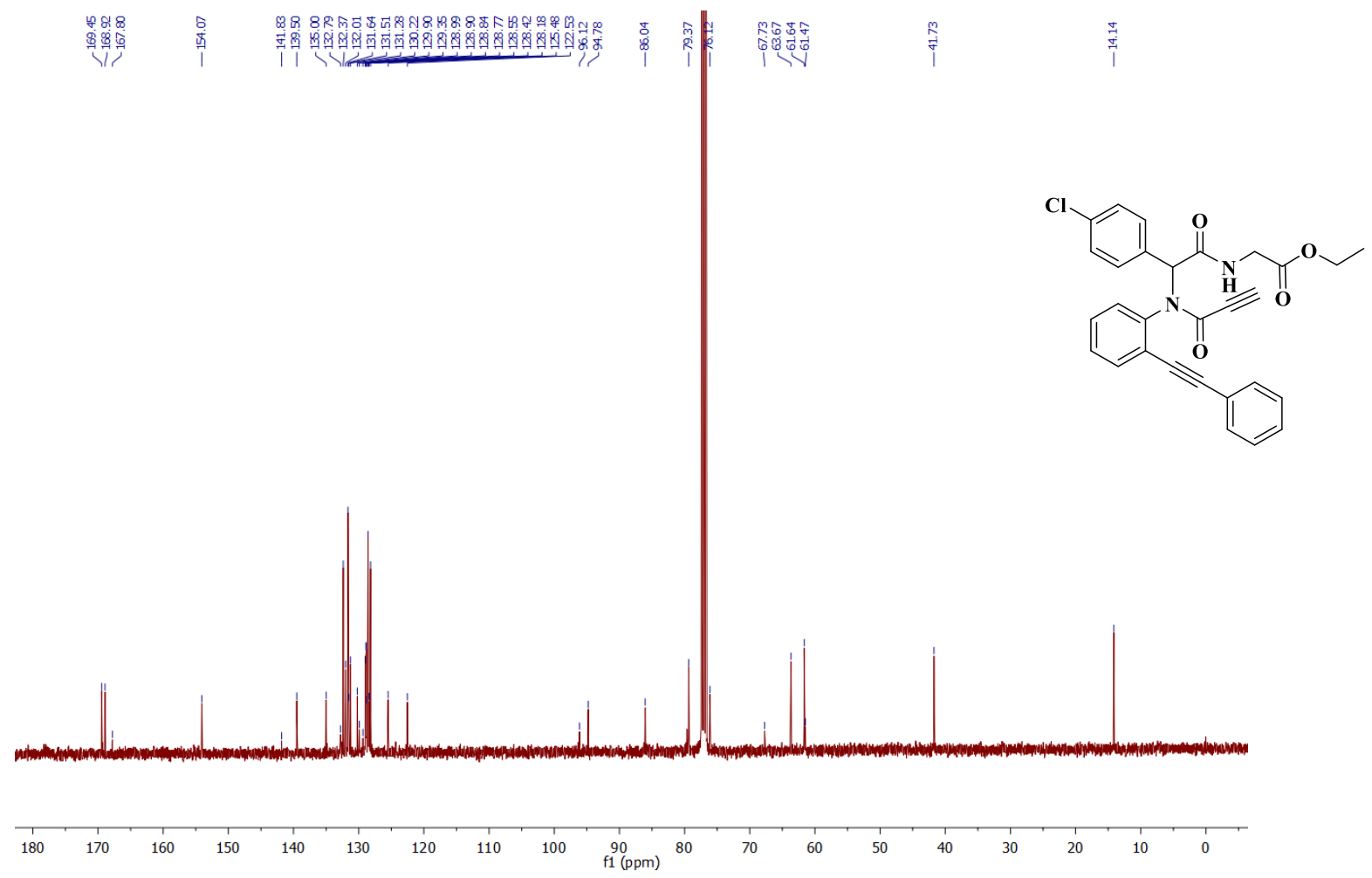

Figure S97. ${ }^{13} \mathrm{C}$ NMR- compound 3i 


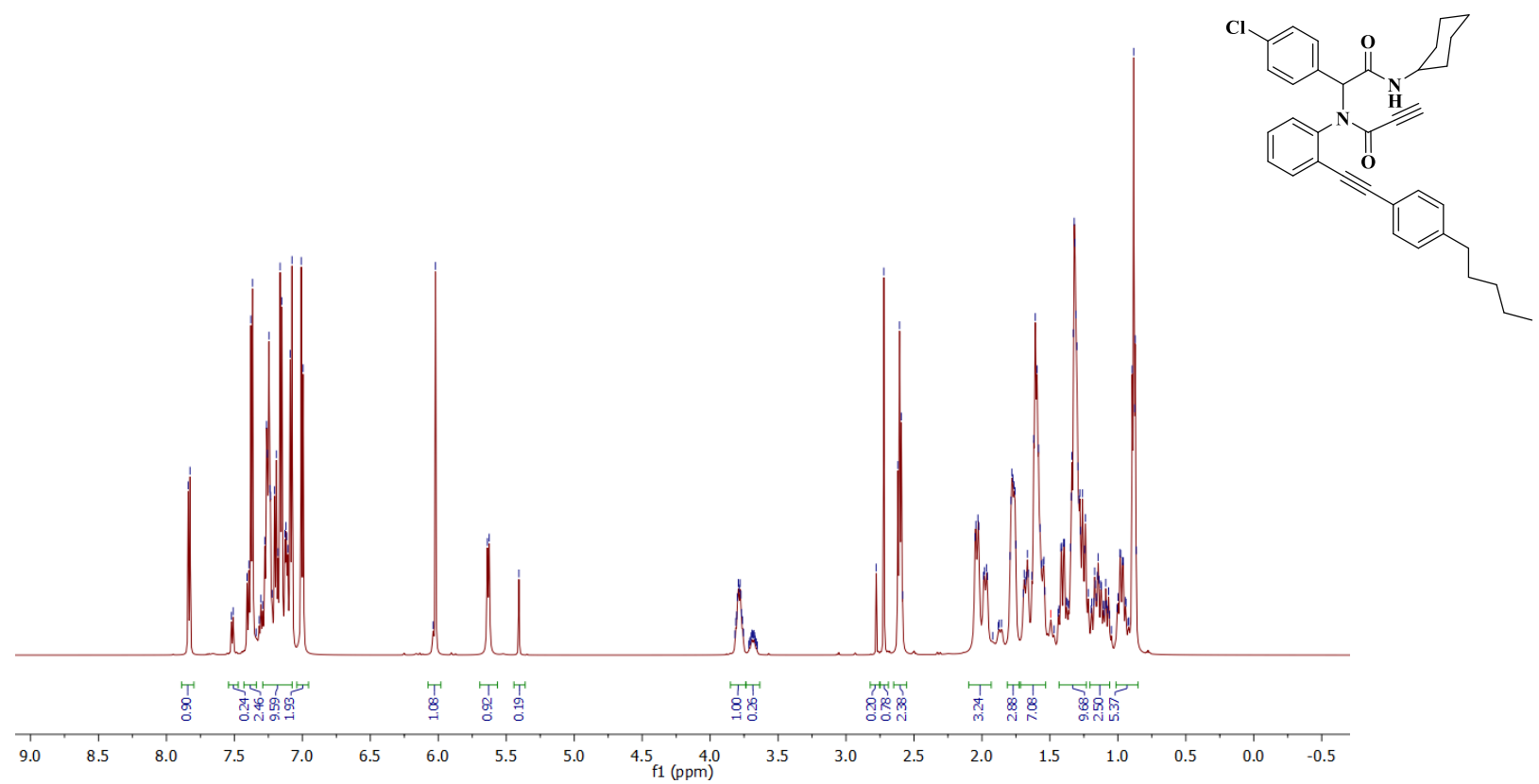

Figure S98. ${ }^{1} \mathrm{H}$ NMR- compound $\mathbf{3 j}$
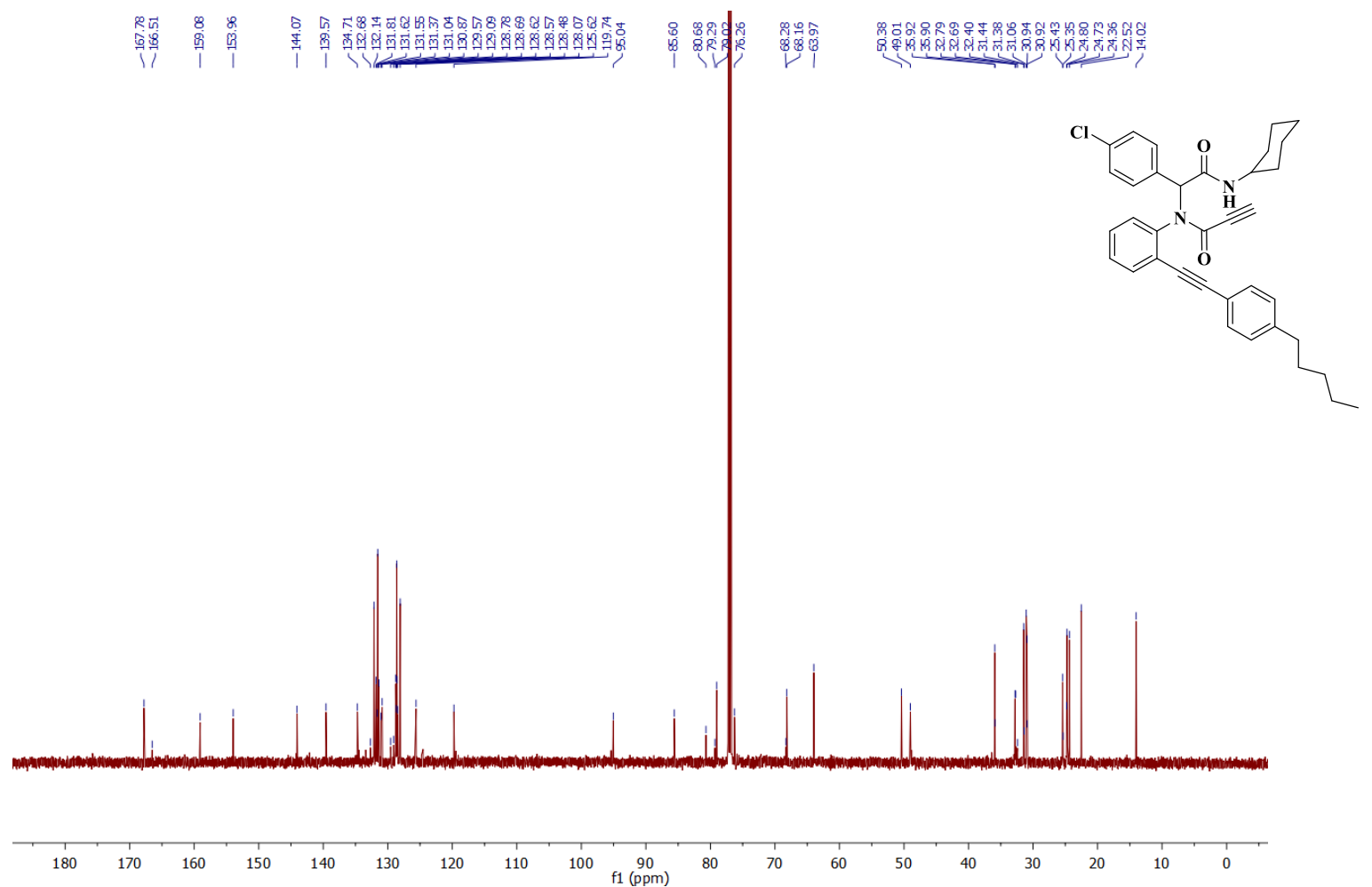

Figure S99. ${ }^{13} \mathrm{C}$ NMR- compound $\mathbf{3 j}$ 


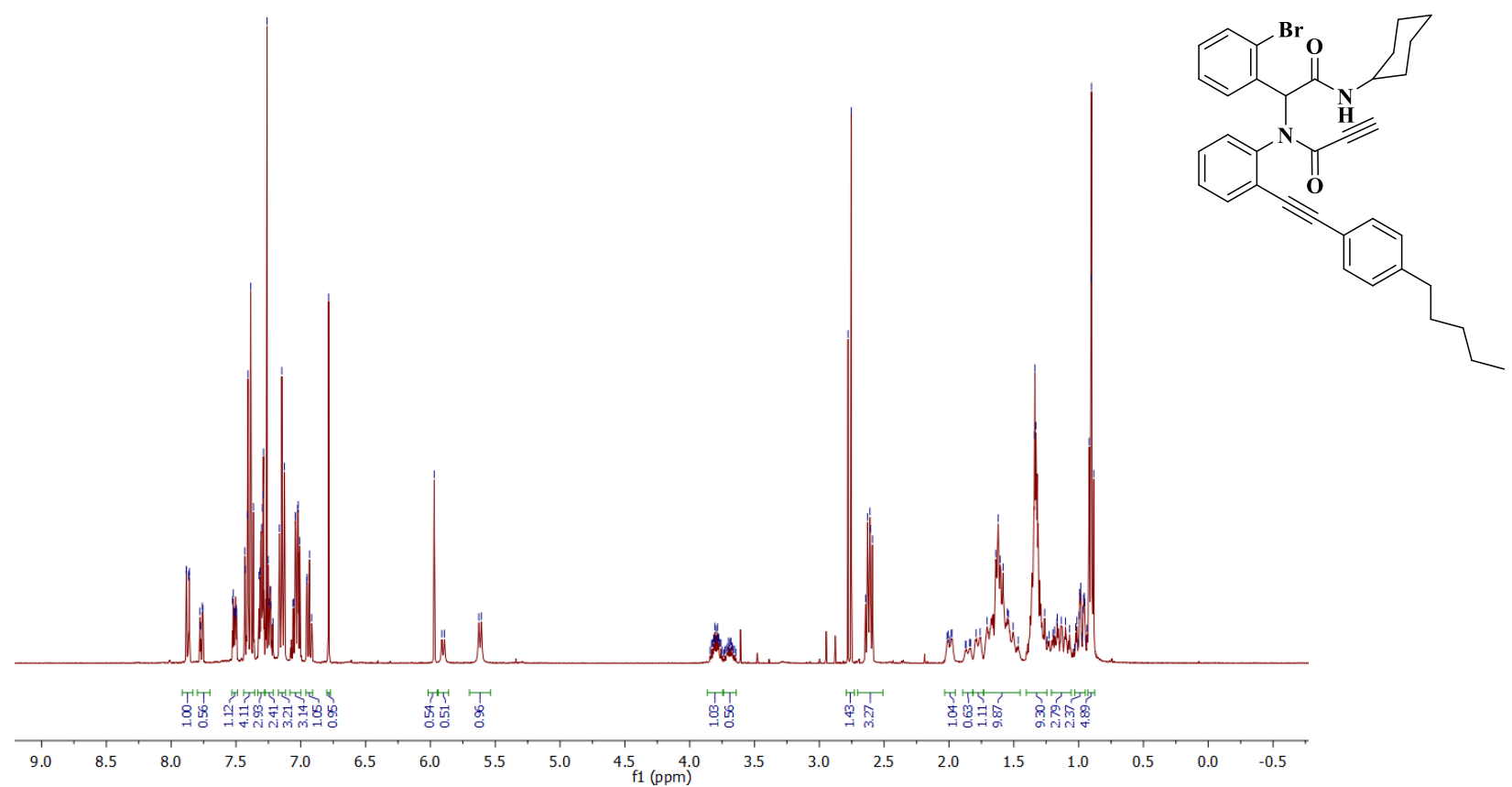

Figure S100. ${ }^{1} \mathrm{H}$ NMR- compound $\mathbf{3 k}$

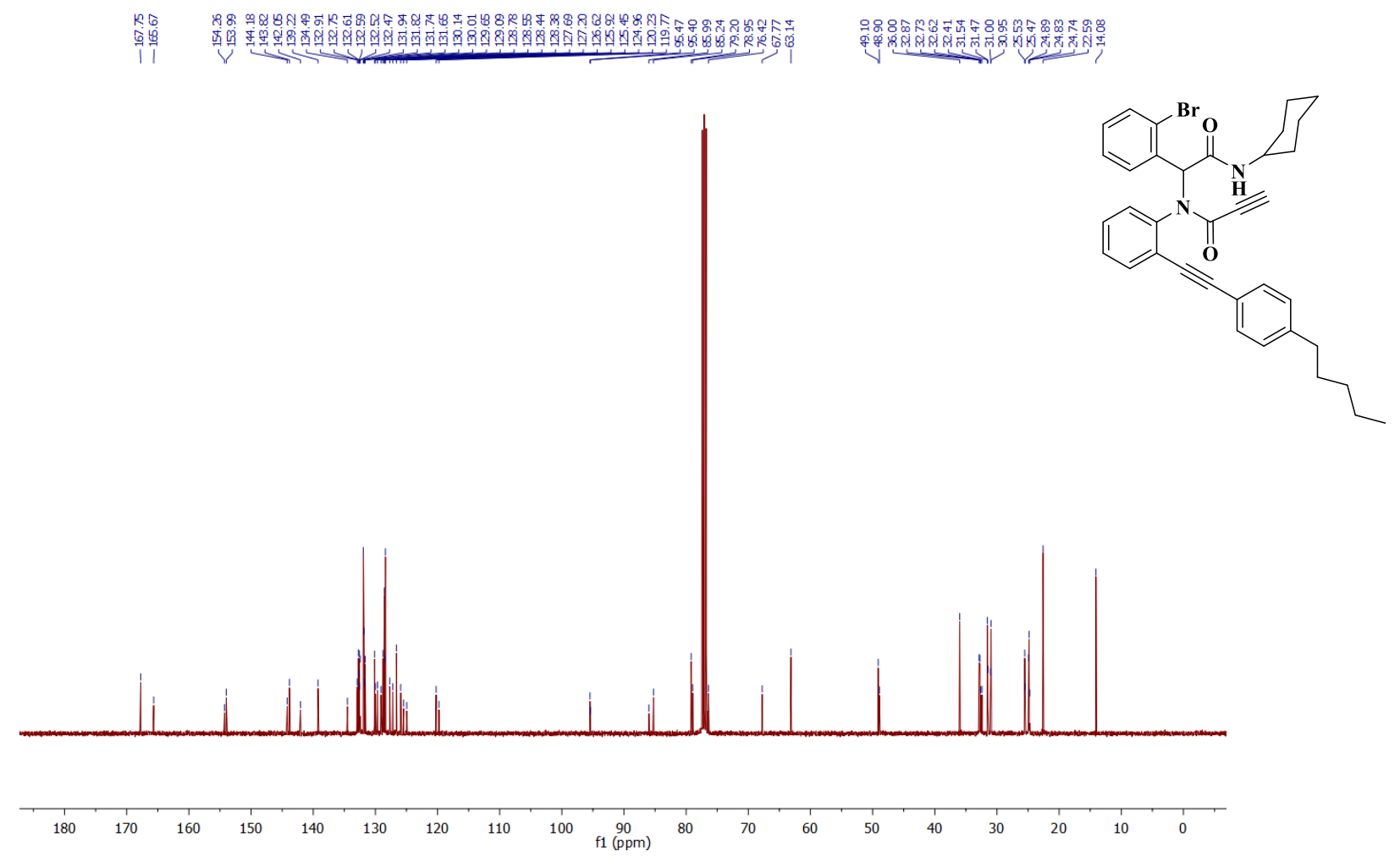

Figure S101. ${ }^{13} \mathrm{C}$ NMR- compound $\mathbf{3 k}$ 


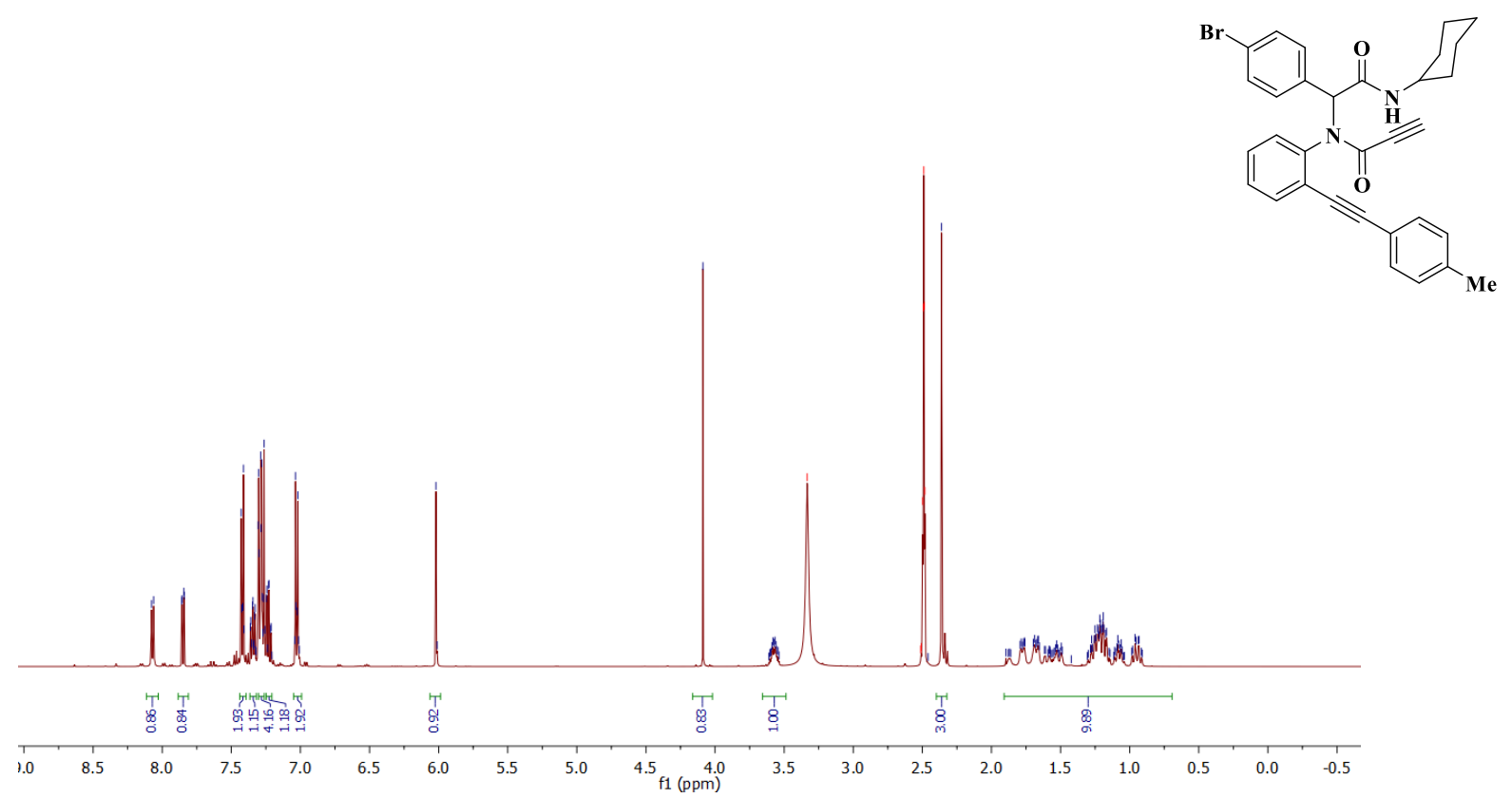

Figure S102. ${ }^{1} \mathrm{H}$ NMR- compound 31

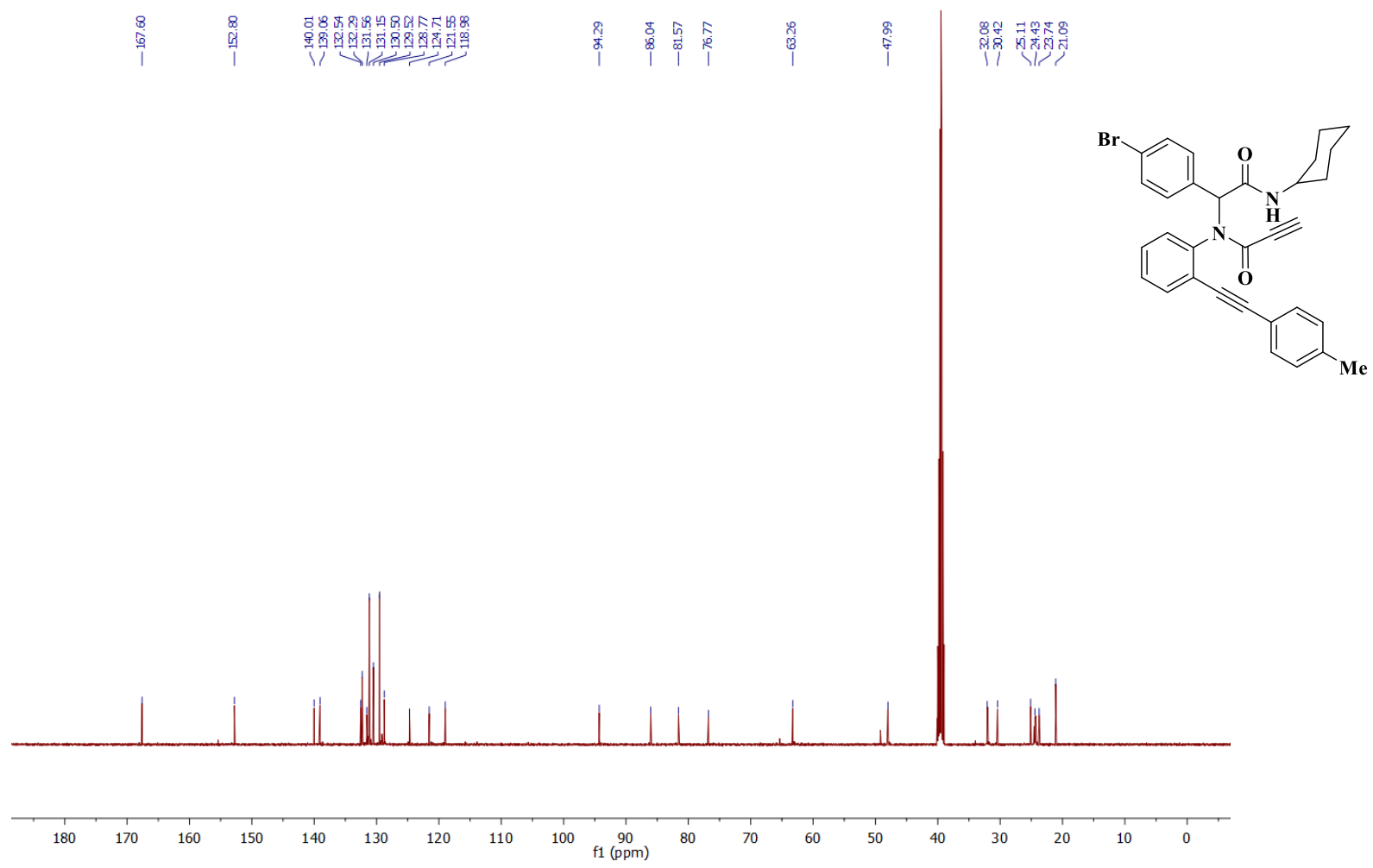

Figure S103. ${ }^{13} \mathrm{C}$ NMR- compound 31 


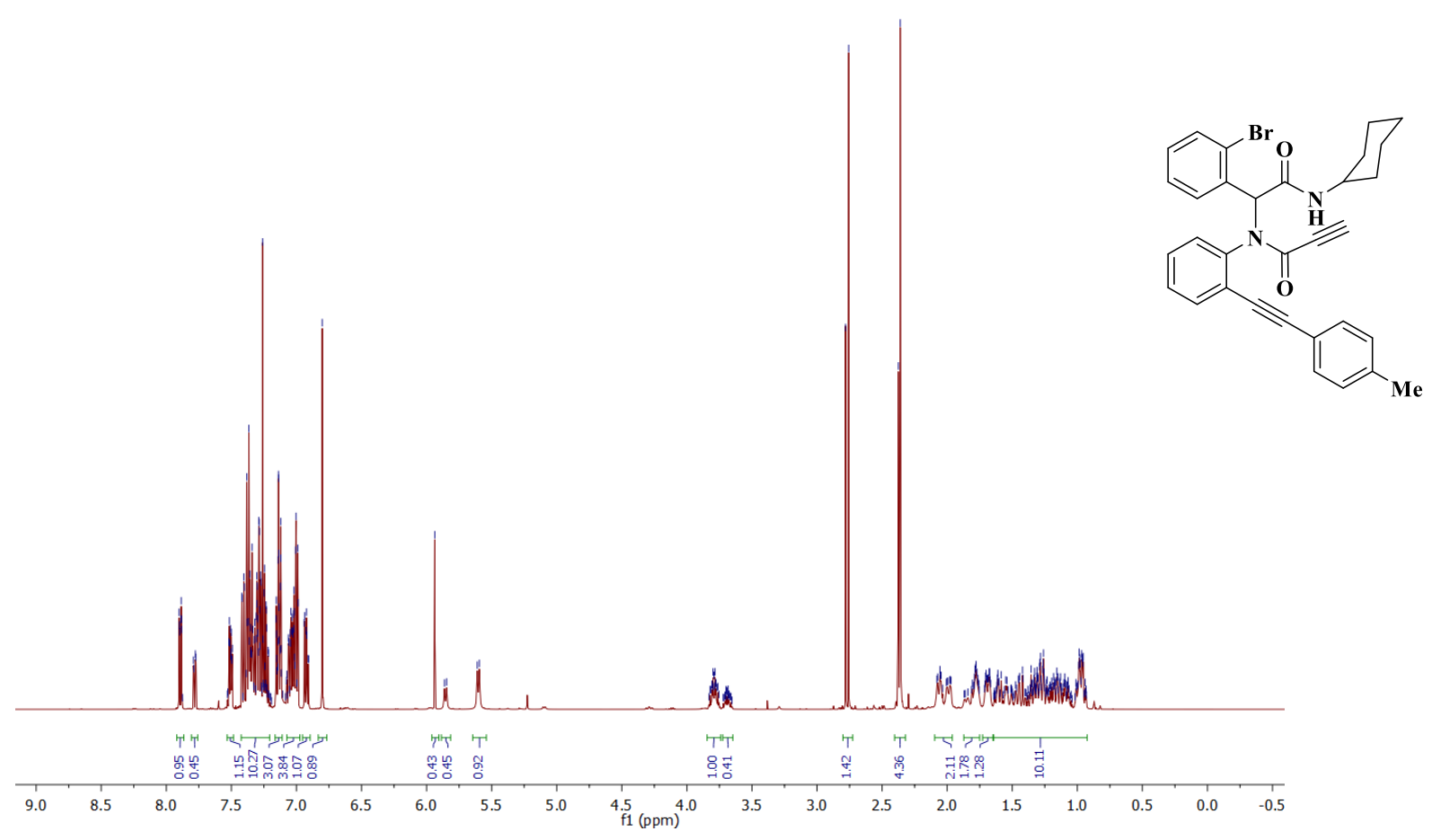

Figure S104. ${ }^{1} \mathrm{H}$ NMR- compound $\mathbf{3 m}$
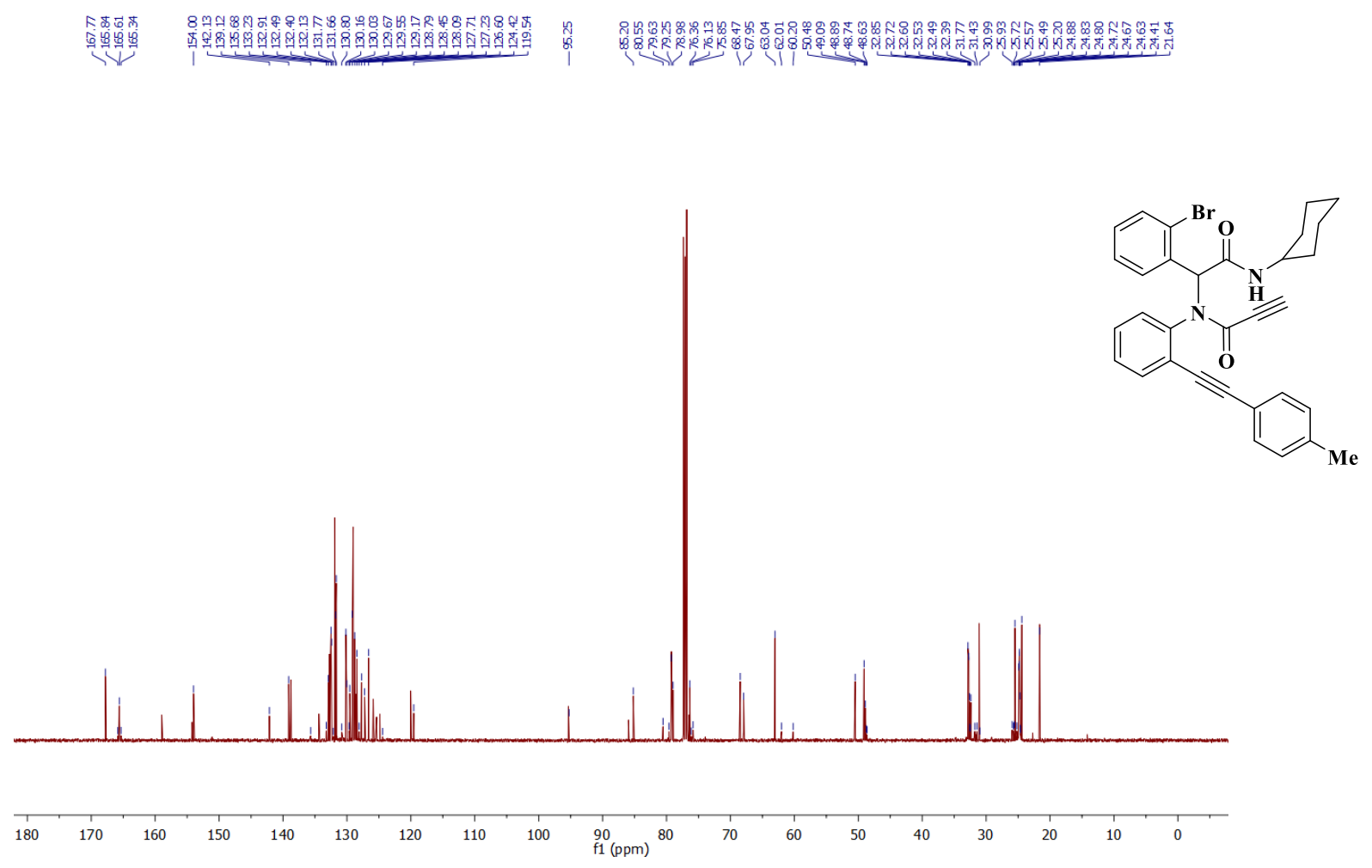

Figure S105. ${ }^{13} \mathrm{C}$ NMR- compound $\mathbf{3 m}$ 


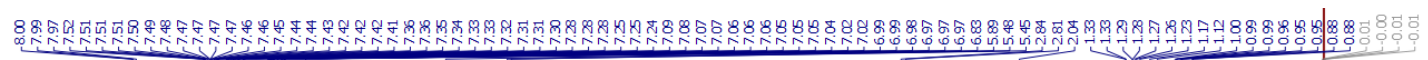<smiles>C#CC(=O)N(c1ccc(Cl)cc1C#Cc1ccccc1)C(C(=O)NC1CCCC1)c1ccccc1Br</smiles>

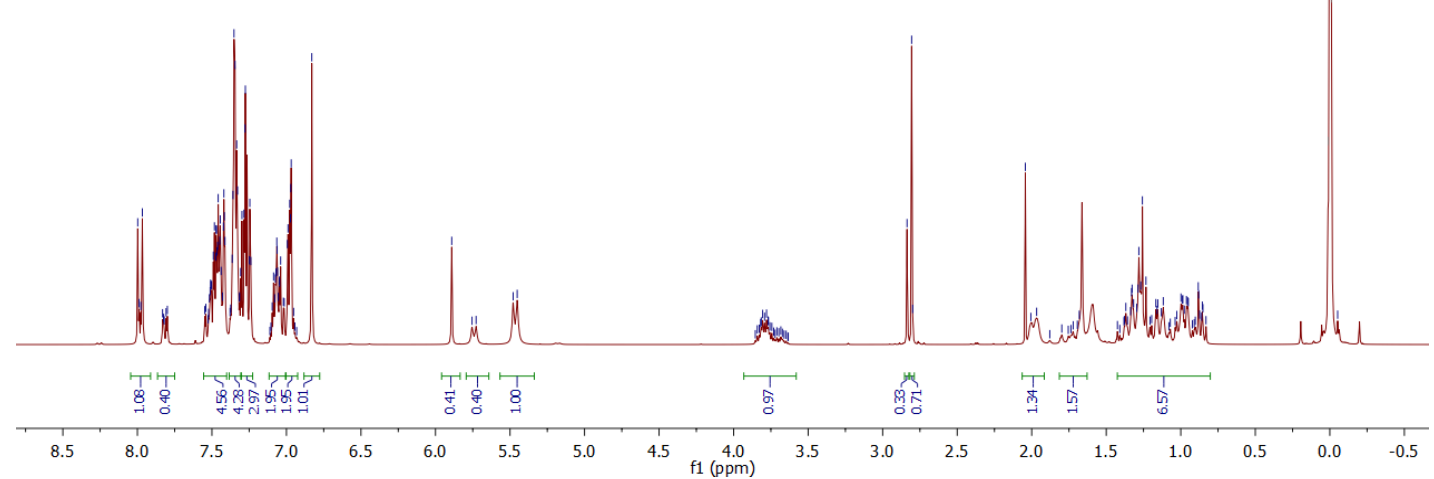

Figure S106. ${ }^{1} \mathrm{H}$ NMR- compound 3n

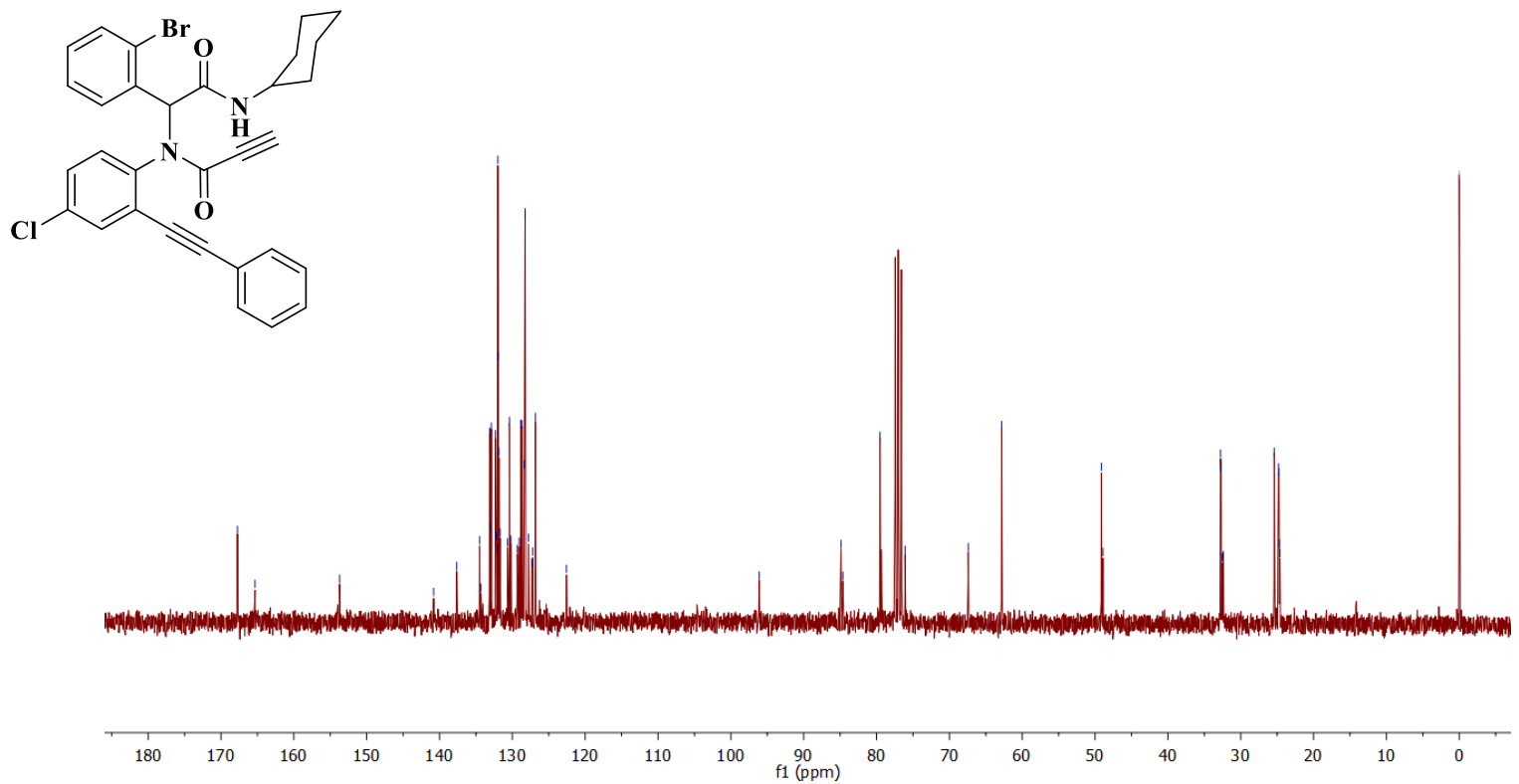

Figure S107. ${ }^{13} \mathrm{C}$ NMR- compound 3n 


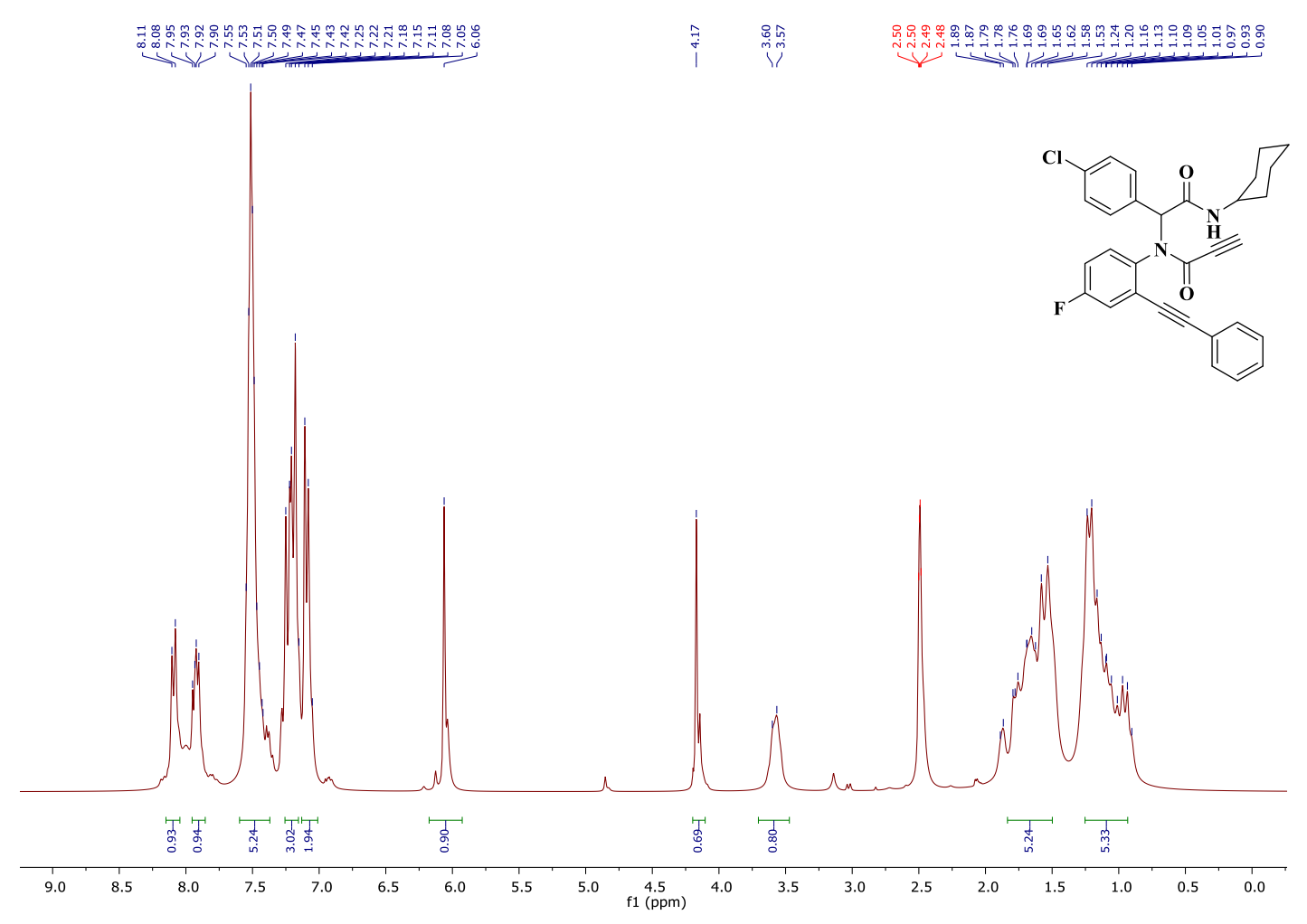

Figure S108. ${ }^{1} \mathrm{H}$ NMR- compound 30

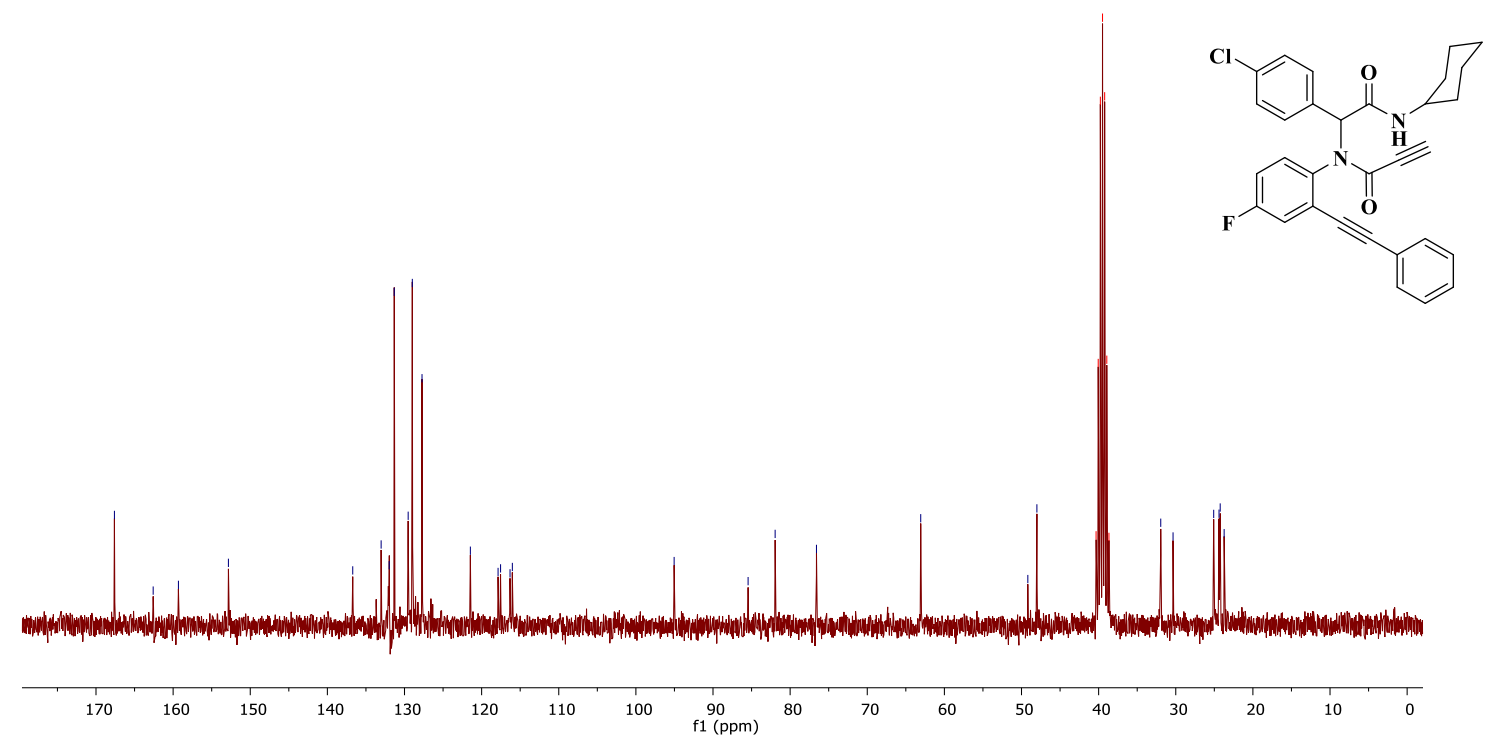

Figure S109. ${ }^{13} \mathrm{C}$ NMR- compound 30 


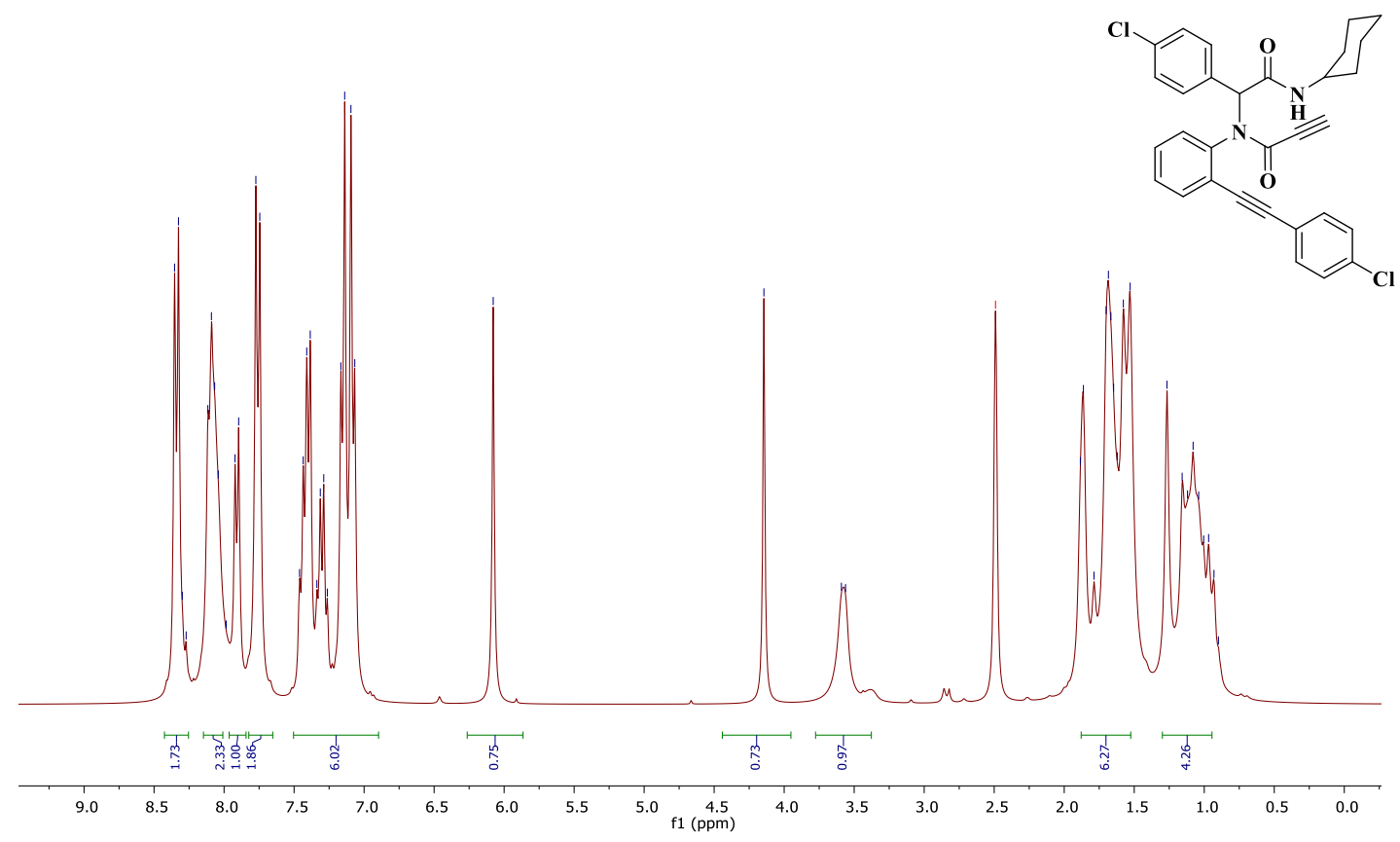

Figure S110. ${ }^{1} \mathrm{H}$ NMR- compound $\mathbf{3 p}$
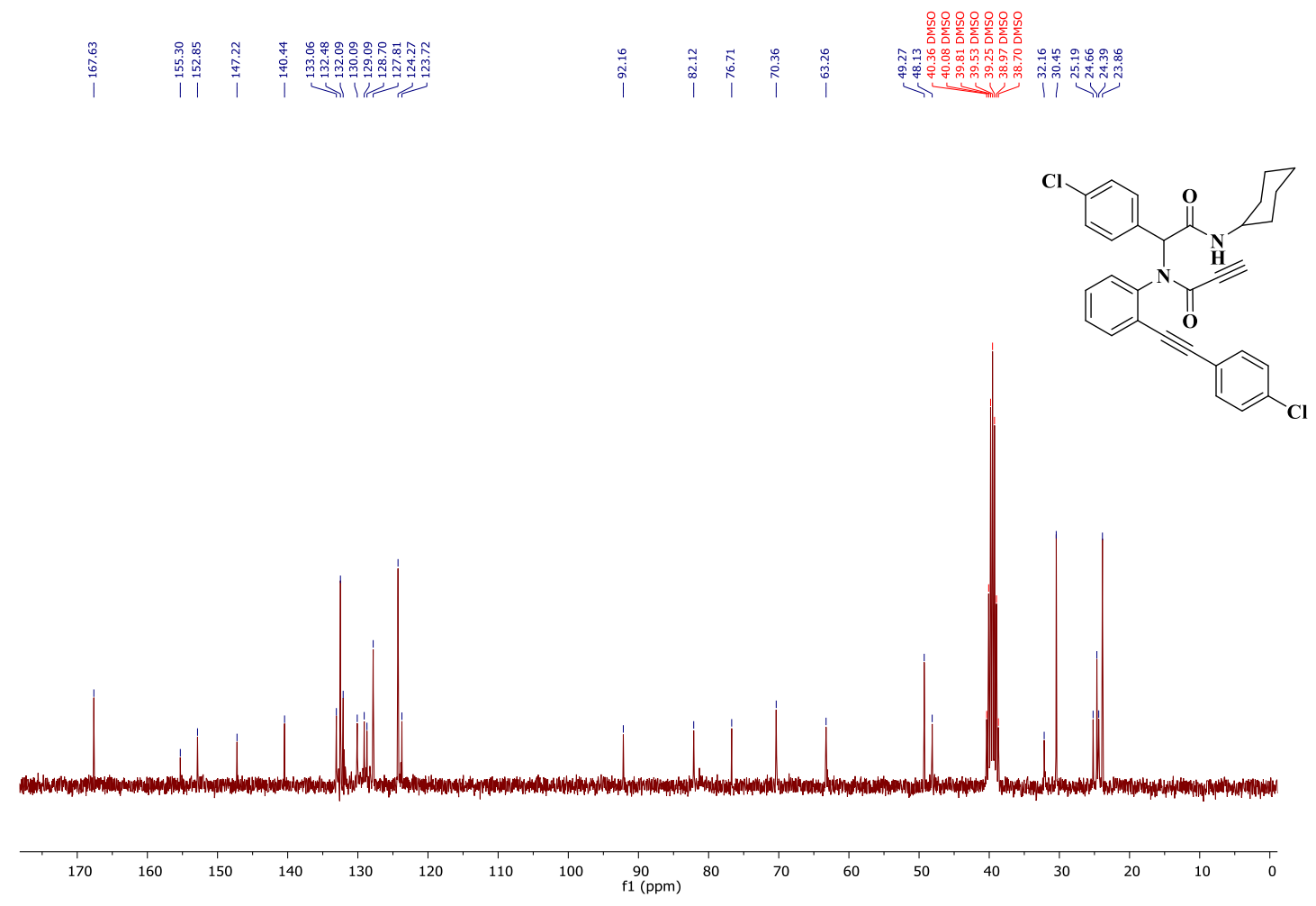

Figure S111. ${ }^{13} \mathrm{C}$ NMR- compound 3p 


\section{Crystallographic Data of 2c and 2h:}

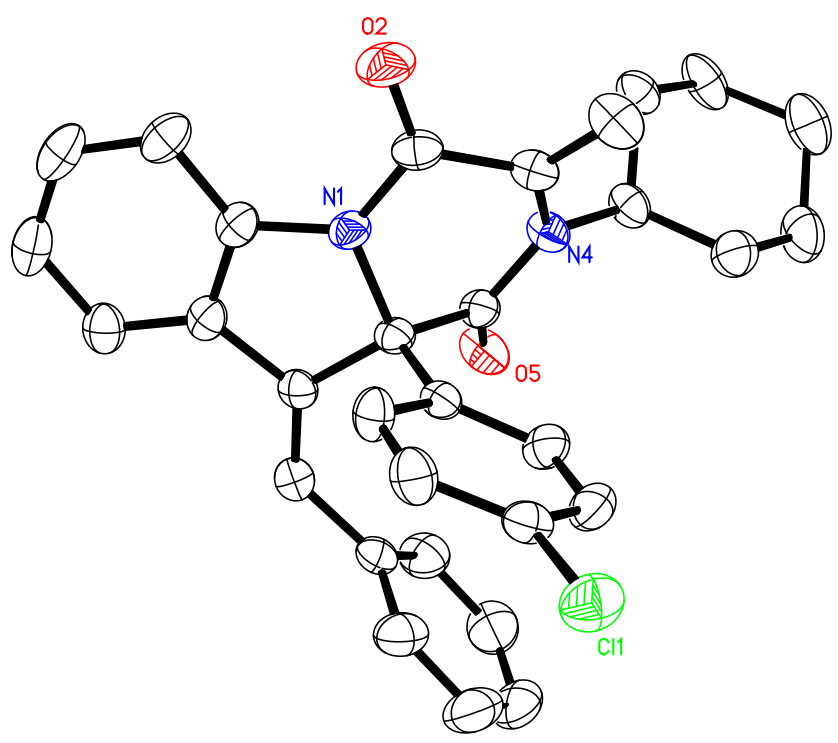

Thermal ellipsoid plot for compound (2c), displacement ellipsoids are drawn at the $50 \%$ probability level.

Crystal Growth: Dichloromethane was used as solvent at room temperature for crystal prepration

sba159 (2c): colourless crystal (brick), dimensions $0.230 \times 0.206 \times 0.105 \mathrm{~mm}^{3}$, crystal system monoclinic, space group $\mathrm{C} 2 / \mathrm{c}, \mathrm{Z}=8, \mathrm{a}=21.532(2) \AA \AA$ $\AA$, alpha $=90$ deg, beta=99.5823(15) deg, gamma=90 deg, $V=4986.2(8) \AA^{3}$, rho=1.319 $\mathrm{g} / \mathrm{cm}^{3}, \mathrm{~T}=200(2) \mathrm{K}$, Thetamax $29.669 \mathrm{deg}$, radiation MoKa, lambda=0.71073 $\mathrm{A}, 0.5 \mathrm{deg}$ omega-scans with CCD area detector, covering the asymmetric unit in reciprocal space with a mean redundancy of 4.33 and a completeness of $99.2 \%$ to a resolution of $0.72 \AA$, 31347 reflections measured, 7004 unique $(R($ int $)=0.0640), 4330$ observed $(I>2 \sigma(I))$, intensities were corrected for Lorentz and polarization effects, an empirical scaling and absorption correction was applied using SADABS based on the Laue symmetry of the reciprocal space, $\mathrm{mu}=0.19 \mathrm{~mm}^{-1}, \mathrm{~T}_{\min }=0.91, \mathrm{~T}_{\max }=0.96$, structure solved with SHELXT2014 (Sheldrick 2014) and refined against $\mathrm{F}^{2}$ with a Full-matrix least-squares algorithm 
using the SHELXL-2018/3 (Sheldrick, 2018) software, 325 parameters refined, hydrogen atoms were treated using appropriate riding models, goodness of fit 1.01 for observed reflections, final residual values $R 1(F)=0.052, w R\left(F^{2}\right)=0.101$ for observed reflections, residual electron density -0.23 to $0.28 \mathrm{e}^{-3}$. CCDC 1954132 contains the supplementary crystallographic data for this paper. These data can be obtained free of charge from The Cambridge Crystallographic Data Centre via https://www.ccdc.cam.ac.uk/structures/.

Table S1: Crystal data and structure refinement for (2c).

\begin{tabular}{|c|c|c|}
\hline Identification code & \multicolumn{2}{|l|}{ sba159 } \\
\hline Empirical formula & \multicolumn{2}{|l|}{$\mathrm{C}_{31} \mathrm{H}_{27} \mathrm{CIN}_{2} \mathrm{O}_{2}$} \\
\hline Formula weight & \multicolumn{2}{|l|}{494.99} \\
\hline Temperature & \multicolumn{2}{|l|}{$200(2) \mathrm{K}$} \\
\hline Wavelength & \multicolumn{2}{|l|}{$0.71073 \AA$} \\
\hline Crystal system & \multicolumn{2}{|l|}{ monoclinic } \\
\hline Space group & \multirow{2}{*}{\multicolumn{2}{|c|}{$\begin{array}{l}\mathrm{C} 2 / \mathrm{c} \\
8\end{array}$}} \\
\hline Z & & \\
\hline Unit cell dimensions & $\begin{array}{l}a=21.532(2) \AA \\
b=8.0393(7) \AA \\
c=29.212(3) \AA\end{array}$ & $\begin{array}{l}\square=90 \text { deg. } \\
\square=99.5823(15) \text { deg. } \\
\square=90 \text { deg. }\end{array}$ \\
\hline Volume & $4986.2(8) \AA^{3}$ & \\
\hline Density (calculated) & $1.32 \mathrm{~g} / \mathrm{cm}^{3}$ & \\
\hline Absorption coefficient & $0.19 \mathrm{~mm}^{-1}$ & \\
\hline Crystal shape & brick & \\
\hline Crystal size & $0.230 \times 0.206 \times 0$ & $\mathrm{~mm}^{3}$ \\
\hline Crystal colour & colourless & \\
\hline Theta range for data collection & 1.4 to 29.7 deg. & \\
\hline Index ranges & $-29 \leq \mathrm{h} \leq 29,-11 \leq \mathrm{k} \leq$ & $-39 \leq 1 \leq 40$ \\
\hline Reflections collected & 31347 & \\
\hline Independent reflections & $7004(\mathrm{R}$ (int) $=0.0$ & \\
\hline Observed reflections & $4330(I>2 \sigma(I))$ & \\
\hline Absorption correction & Semi-empirical frc & quivalents \\
\hline Max. and min. transmission & 0.96 and 0.91 & \\
\hline Refinement method & Full-matrix least-s & res on $\mathrm{F}^{2}$ \\
\hline Data/restraints/parameters & 7004 / 0 / 325 & \\
\hline Goodness-of-fit on $\mathrm{F}^{2}$ & 1.01 & \\
\hline Final $R$ indices (I>2sigma(I)) & $\mathrm{R} 1=0.052, \mathrm{wR} 2$ & 101 \\
\hline Largest diff. peak and hole & 0.28 and -0.23 e & \\
\hline
\end{tabular}

Crystal structure data for $\mathbf{2 h}$

Table S2: Crystal data and structure refinement for sba164.

Identification code Empirical formula

Formula weight sba164

$\mathrm{C}_{31} \mathrm{H}_{27} \mathrm{BrN}_{2} \mathrm{O}_{2}$

539.45 


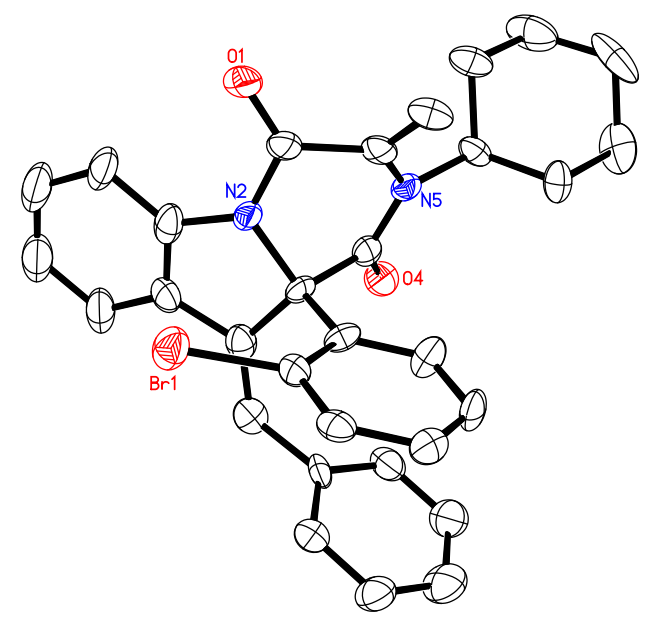

Thermal ellipsoid plot for compound (2h), displacement ellipsoids are drawn at the $50 \%$ probability level.

Crystal Growth: Dichloromethane was used as solvent at room temperature for crystal prepration

sba164 (2h): colourless crystal (needle), dimensions $0.162 \times 0.044 \times 0.028 \mathrm{~mm}^{3}$, crystal system orthorhombic, space group $P 212121, Z=4, a=15.5188(10) \AA, b=21.4989(13) \AA$, $\mathrm{C}=7.5658(5) \AA$, alpha $=90 \mathrm{deg}$, beta=90 deg, gamma $=90 \mathrm{deg}, \mathrm{V}=2524.2(3) \AA^{3}$, rho=1.419 $\mathrm{g} / \mathrm{cm}^{3}, \mathrm{~T}=110(2) \mathrm{K}$, Theta $\max =67.035 \mathrm{deg}$, radiation $\mathrm{CuK} \alpha$, lambda $=1.54178 \AA, 0.5 \mathrm{deg}$ omega-scans with CCD area detector, covering the asymmetric unit in reciprocal space with a mean redundancy of 3.41 and a completeness of $97.4 \%$ to a resolution of $0.84 \AA$, 8845 reflections measured, 4003 unique $(R($ int $)=0.0604), 2340$ observed $(I>2 \sigma(I))$, intensities were corrected for Lorentz and polarization effects, an empirical scaling and absorption correction was applied using X-Area LANA 1.70.0.0 (STOE, 2017) based on the Laue symmetry of the reciprocal space, $m u=2.46 \mathrm{~mm}^{-1}, T_{\min }=0.71, T_{\max }=1.62$, structure solved with SHELXT-2014 (Sheldrick 2014) and refined against $\mathrm{F}^{2}$ with a Fullmatrix least-squares algorithm using the SHELXL-2018/3 (Sheldrick, 2018) software, 578 parameters refined, hydrogen atoms were treated using appropriate riding models, Flack absolute structure parameter $0.46(4)$, goodness of fit 0.99 for observed reflections, final 
residual values $R 1(F)=0.058, w R\left(F^{2}\right)=0.123$ for observed reflections, residual electron density -0.36 to $0.28 \mathrm{e}^{-3}$. CCDC 1954133 contains the supplementary crystallographic data for this paper. The data can be obtained free of charge from The Cambridge Crystallographic Data Centre via www.ccdc.cam.ac.uk/structures.

\section{References :}

1. (a) Krause, L.; Herbst-Irmer, R.; Sheldrick, G. M.; Stalke, D. Comparison of Silver and Molybdenum Microfocus X-ray Sources for Single-Crystal Structure Determination. J. Appl. Cryst. 2015, 48, 3-10. (b) Sheldrick, G. M. Shelxt Integrated Space-Group and Crystal Structure Determination. Acta Cryst. 2015, A71, 3-8. 